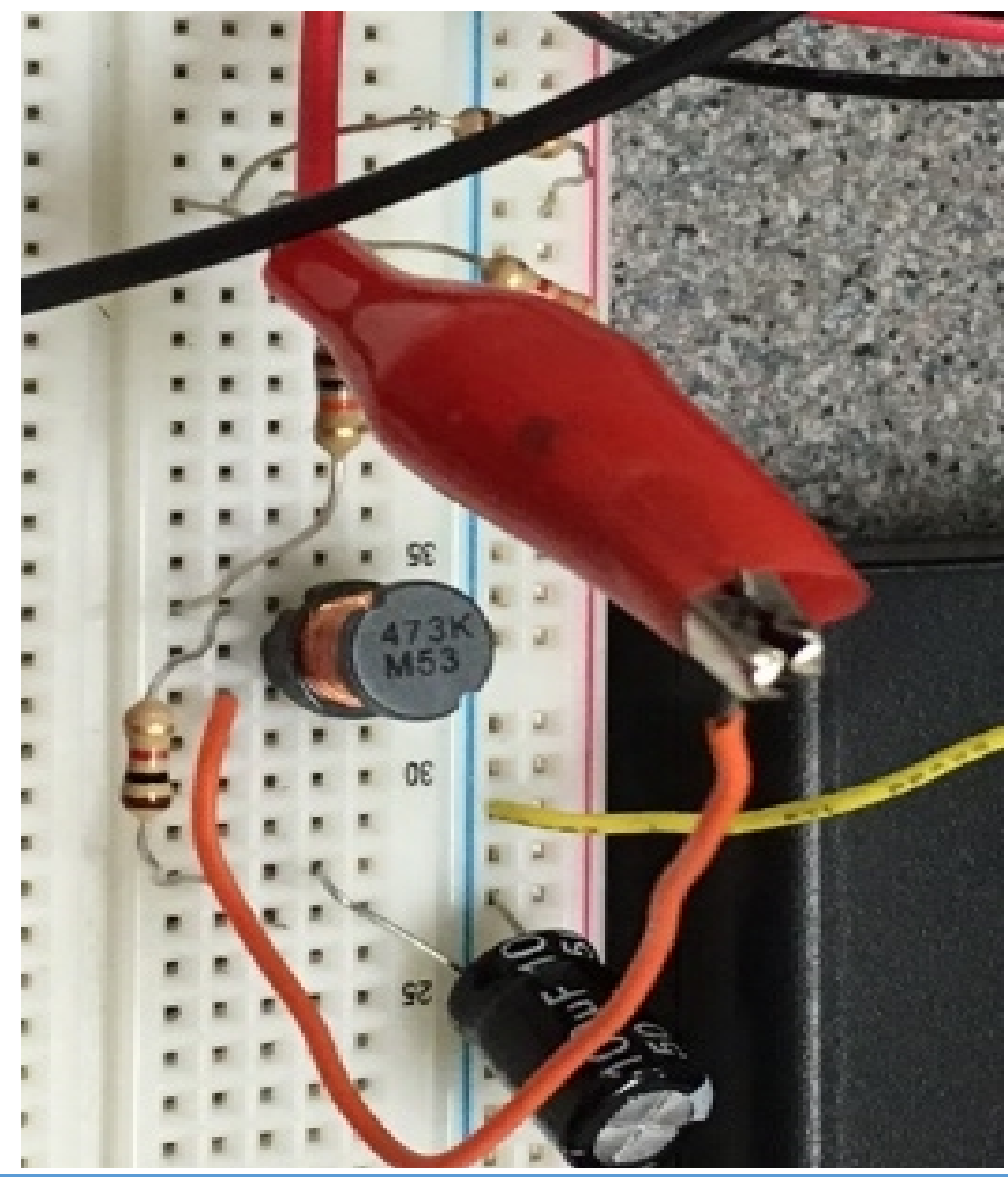

\title{
AC Circuits
}

1st Edition 
How to navigate this book: This document contains bookmark links, which are links to other locations in this document. These are not the same as website links, which will open up a webpage in your default browser. If you click on a bookmark and want to go back to where you were previously at in the document, click on ALT - Left Arrow $(\leftarrow)$.

For example:

- This is a bookmark to the Preface section $\rightarrow$ Preface

- This is a website link to the DC Circuits pre-requisite book.

Getting the Latest Version of this book: If you see any errors or typos please go to my website (ChadDavis.oucreate.com) and enter them in the comments section and I will get the book updated. Minor changes in the book will not result in a new version number, but instead will be corrected and uploaded to the following webpage:

https://shareok.org/handle/11244/51946

If you have any suggestions for significant changes that could potentially be implemented into a new edition in the future, I would also appreciate that type of feedback on my website.

(C) Chad Davis, PhD, PE 2017

CC BY-NC-SA

This book was created by Chad E. Davis and released under the Creative Commons License. 


\section{Table of Contents}

Preface .4

Module 1 - AC Signal Overview

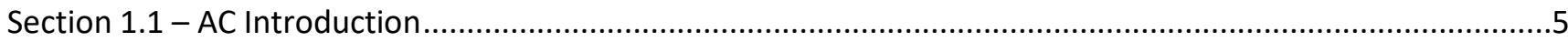

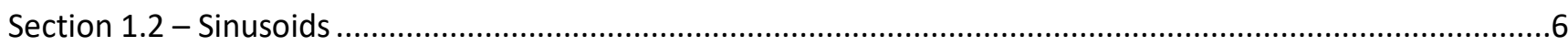

Section 1.3- Defining AC Voltage Types for Common AC Waveforms …................................................... 12

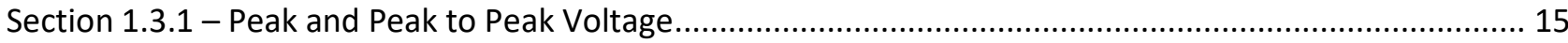

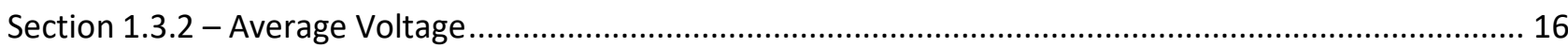

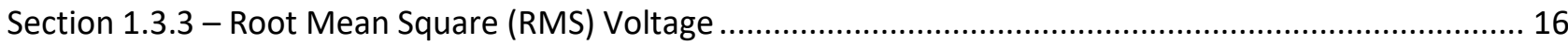

Section 1.4- Sine Wave, Square Wave, and Triangle Wave Example Problems ........................................... 18

Section 1.4.1 - Example Calculations of Vpp, Vpk, Vavg, and Vrms for Sine Waves ................................... 18

Section 1.4.2 - Example Calculations of Vpp, Vpk, Vavg, and Vrms for Square Waves............................... 20

Section 1.4.3 - Example Calculations of Vpp, Vpk, Vavg, and Vrms for Triangle Waves ............................... 23

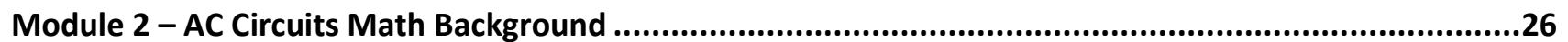

Module 3 - RLC Circuit Analysis with AC Sources..............................................................................32

Section 3.1 - Introduction of Impedance and Admittance and the Laplace Transform ................................. 32

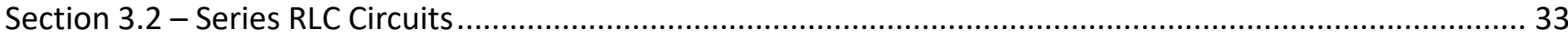

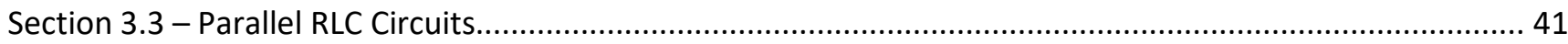

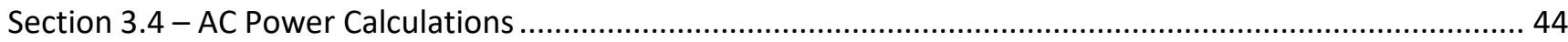

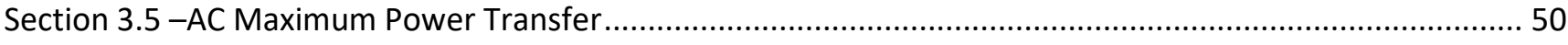

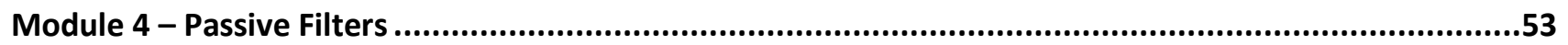

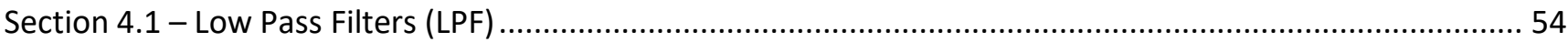

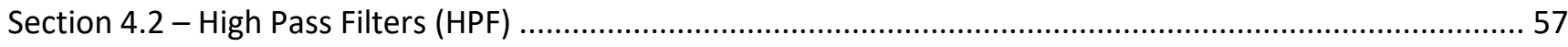

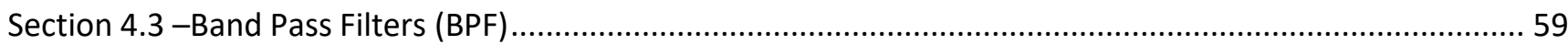

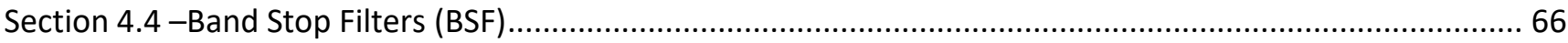

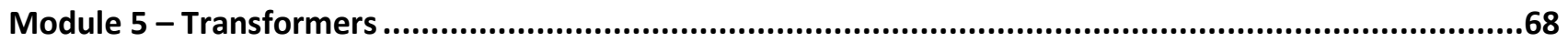

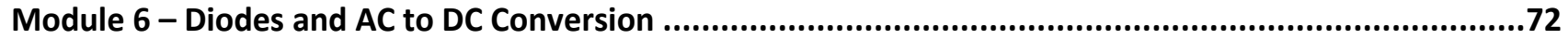

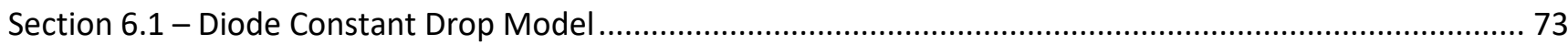

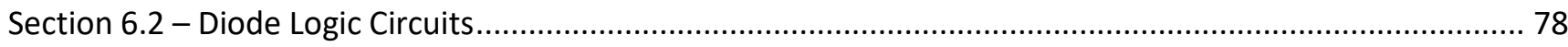

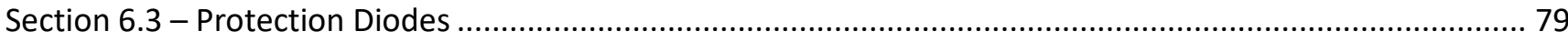

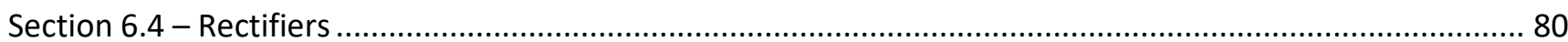

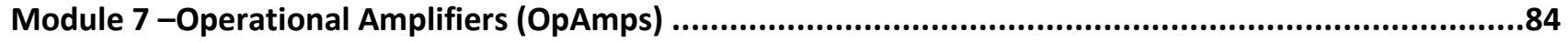

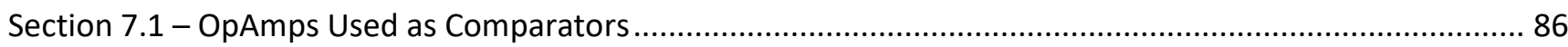

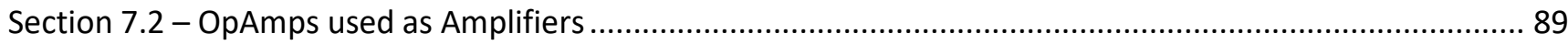

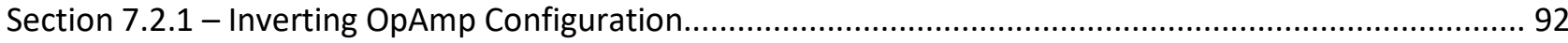

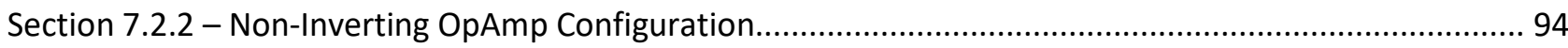

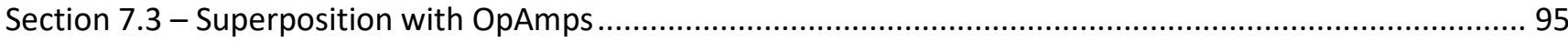

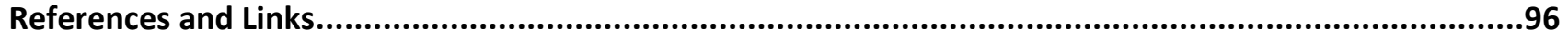

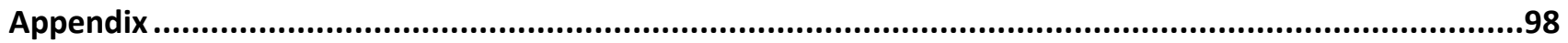




\section{Preface}

This eBook was written as the sequel to the eBook titled DC Circuits, which was written in 2016 by Chad Davis. The DC Circuits eBook (https://shareok.org/handle/11244/52245) should be reviewed prior to reading this book. While the first book covered only DC circuits, this book covers Alternating Current (AC) circuits as well as a brief introduction of electronics. It is broken up into seven modules. Module 1 covers the theory of AC signals. Since only DC sources are used in the first eBook, details of AC signals such as sinusoidal waveforms (or sine waves), square waves, and triangle waves are provided. Module 2, titled AC Circuits Math Background, covers the mathematics needed for solving AC circuit problems. The background material in Modules 1 and 2 are combined in Module 3 to solve circuits with AC sources with resistors, inductors, and capacitors (RLC circuits).

Note: The term RLC circuit will apply to circuits that contain resistors and capacitor and/or inductors in this eBook. Therefore, the RLC term will also apply to RL and RC circuits.

Module 4 focuses on using RLC circuits as passive filters. Content that is traditionally associated with AC Circuits material ends in Module 4, but additional content is included in Modules 5 to 7 to provide more practical knowledge that builds upon the theory learned in AC circuits. Topics in these final three modules include transformers, diodes, and operational amplifiers. These topics in Modules 5 to 7 are the foundation of the field of electronics. Only an overview is provided in this eBook, but if it peaks your interest http://www.allaboutcircuits.com/textbook/semiconductors/ has a detailed open source eBook on electronics.

An important thing to realize is that most of the information covered in DC circuit theory also applies to AC circuit theory. In fact, it is a good idea to think of a DC signal as one that can be obtained by freezing time in an AC signal and looking at the "instantaneous" voltage or current values at that moment in time. With this in mind, it should be apparent that KVL, KCL, Ohm's law, and all of the other primary equations used in DC circuits also apply to AC circuits, since the AC circuit is simply a DC circuit that continually changes values. While DC voltage or current signals are typically given capital letter variables ( $\mathrm{V}$ or I), AC waveforms are given lower case variables and often written as a function of time, $\mathrm{v}(\mathrm{t})$ or $\mathrm{i}(\mathrm{t})$. In this eBook scalar values that define the different types of AC voltages and currents are given capital letters (Vpk, Ipk, Vpp, Ipp, Vavg, lavg, Vrms, Irms, and A) as defined in Module 1.

One goal of this book is to provide practical information to better equip the reader to put circuit theory into practice. The picture on the cover of this book shows an example of a RLC circuit built on a breadboard. After the completion of this book you will understand how RLC circuits work and many of the applications they are used for, as well as obtain a foundation in electronics. While providing practical information, this eBook tries to avoid going into too many tangents that result in numerous pages being skipped over by the reader. Links are provided throughout the eBook where more background information is available when I felt the temptation to depart from the main point. Many of the links come from allaboutcircuits.com, which is a very good open educational resource. If a link doesn't open copy it into your browser or search for the document title online.

The circuits in this eBook are created with a user friendly circuit drawing and simulation tool called Multisim. Creating new circuits and simulating them to verify that your calculations are correct is a good way to hone your circuit analysis skills. By providing an explanation of how to verify calculations in Multisim, this eBook enables the reader to create more complicated problems, while having a method to check the results. This is vitally important with AC circuits because the complex algebra involved in solving them is very tedious and mistakes are frequently made by students that are learning the problem solving techniques. The DC probes and Multimeters were used extensively in the DC Circuits eBook, but in this eBook AC probes and the MultiSim oscilloscope will also be used to make more complicated AC measurements. 


\section{Module 1 - AC Signal Overview}

Module 1 is broken up into the following 4 sections.

- Section 1.1-AC Introduction

- Section 1.2-Sinusoids

- Section 1.3 - Common AC Waveforms and Voltage Types

- Section 1.4 - Sine Wave, Square Wave, and Triangle Wave Example Problems

\section{Section 1.1 - AC Introduction}

When analyzing Direct Current (DC) circuits, calculations and measurements were simplified because the currents and voltages remained at a constant value when the circuit was at steady state. For Alternating Current (AC) circuits, the steady state voltage and current fluctuates in such a manner that the current periodically changes directions in the circuit. With the signals in the circuit being much more complicated, the analysis of $A C$ circuits is also at a higher complexity level. An important thing to realize is that most of the information covered in DC circuit theory also applies to AC circuit theory. In fact, it is a good idea to think of a DC signal as one that can be obtained by freezing time in an AC signal and looking at the instantaneous voltage or current values at that specified time. With this in mind, it should be apparent that KVL, KCL, Ohm's law, and all of the other primary equations used in DC circuits also apply to AC circuits since the AC circuit is only a DC circuit that continually changes values. While DC voltage or current signals are given capital letter variables (V or I), AC signals are given lower case variables and written as a function of time, $\mathrm{v}(\mathrm{t})$ or $\mathrm{i}(\mathrm{t})$. In this eBook scalar values that define the different types of AC voltages and currents are given capital letters (Vpk, Ipk, Vpp, Ipp, Vavg, lavg, Vrms, Irms, and A). These AC parameters are defined in sections 1.2 to 1.4.

Note: In the DC Circuits eBook Figure 1.7a showed how to change the probe settings in MultiSim and described each of the "periodic" probe measurements. For convenience, the explanation and Figure (see Figure 1.0) is repeated here. When the current and voltage probes are normally placed in Multisim the "Instantaneous and periodic" probe setting is the default so you will see 5 voltage measurements (as shown in Figure 1.0). From top to bottom these measurements are: 1-Instantaneous, 2-peak to peak (Vpp or Ipp), 3-Root-Mean-Square (Vrms or Irms), 4-average (Vavg or lavg), and 5-frequency of the signal. The average voltage is labeled V(dc) in the Multisim probe because the average voltage is equivalent to the DC value. The instantaneous voltage is the value at a specific time and will continuously change as the AC current flows through the circuit. These four additional parameters for voltage or current are only used for AC circuits. When dealing with DC circuits there is only one type of voltage and current so only the instantaneous measurements are needed. To get only the DC current or voltage click on the probe settings and change the default to "Instantaneous Only" as shown in Figure $\underline{1.0 .}$.
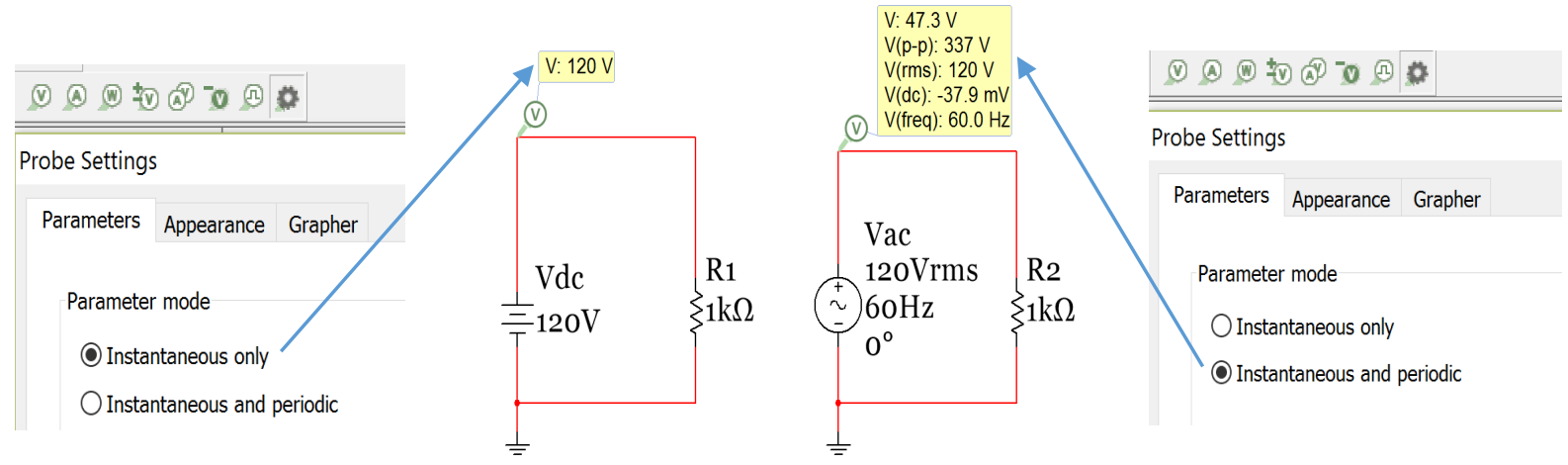

Figure 1.0: Preferred probe settings for DC circuits (Left) and AC circuits (Right). 
Before analyzing AC circuits it is good to have a brief history of DC and AC circuits. This link (http://www.allaboutcircuits.com/news/why-is-the-us-standard-60-hz/) provides some DC and AC background and discusses how the United States (US) power system standardized on $60 \mathrm{Hertz}(\mathrm{Hz})$ sinusoidal signals and Europe, and most of the rest of the world, standardized on $50 \mathrm{~Hz}$ sinusoidal signals (Sinusoids are discussed in detail in Section 1.2). This eBook will focus primarily on $60 \mathrm{~Hz}$ signals. The voltage levels used in AC circuits is also an important factor. US most commonly uses a Root Mean Square (RMS - discussed in detail in Section 1.3) voltage level between 110 and 120 Volts. 110 Vrms and 120 Vrms are often used interchangeably to describe the same signal. In the previous link $110 \mathrm{Vrms}$ is used, but $120 \mathrm{Vrms}$ is a more commonly referred to value and therefore $120 \mathrm{Vrms}$ will be used for the amplitude of a standard AC outlet voltage signal in this eBook. According to this link the average RMS voltage is really around 117 Vrms.

Figure 1.1 shows a Multimeter in AC mode measuring the RMS voltage of a standard US electrical outlet. In this case, the measurement is slightly higher than $120 \mathrm{Vrms}$. Figure 1.2 shows how a 120 VRMS, $60 \mathrm{~Hz}$ voltage source would appear if plotted on an oscilloscope. Notice, that the top AC outlet in Figure 1.1 is concealed with a safety cover to avoid an accidental shock. One important note about $A C$ is that it is usually considered much

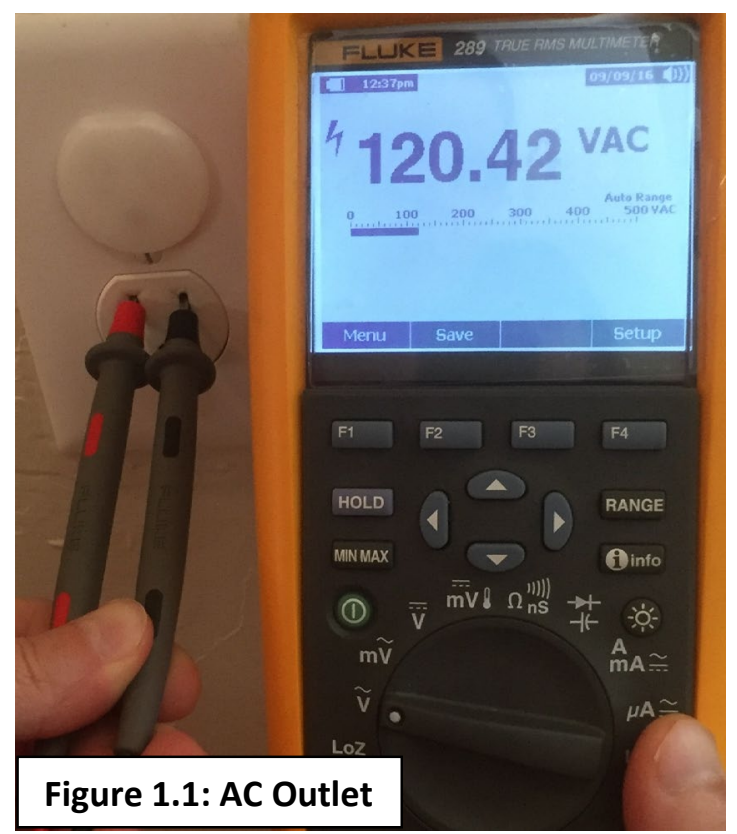
more dangerous than DC for many reasons. The following link discussed electrical safety in great detail and provides data that shows that a $60 \mathrm{~Hz}$ AC signals can result in heart fibrillation at much lower currents than DC. http://www.allaboutcircuits.com/textbook/direct-current/chpt-3/ohms-law-again/

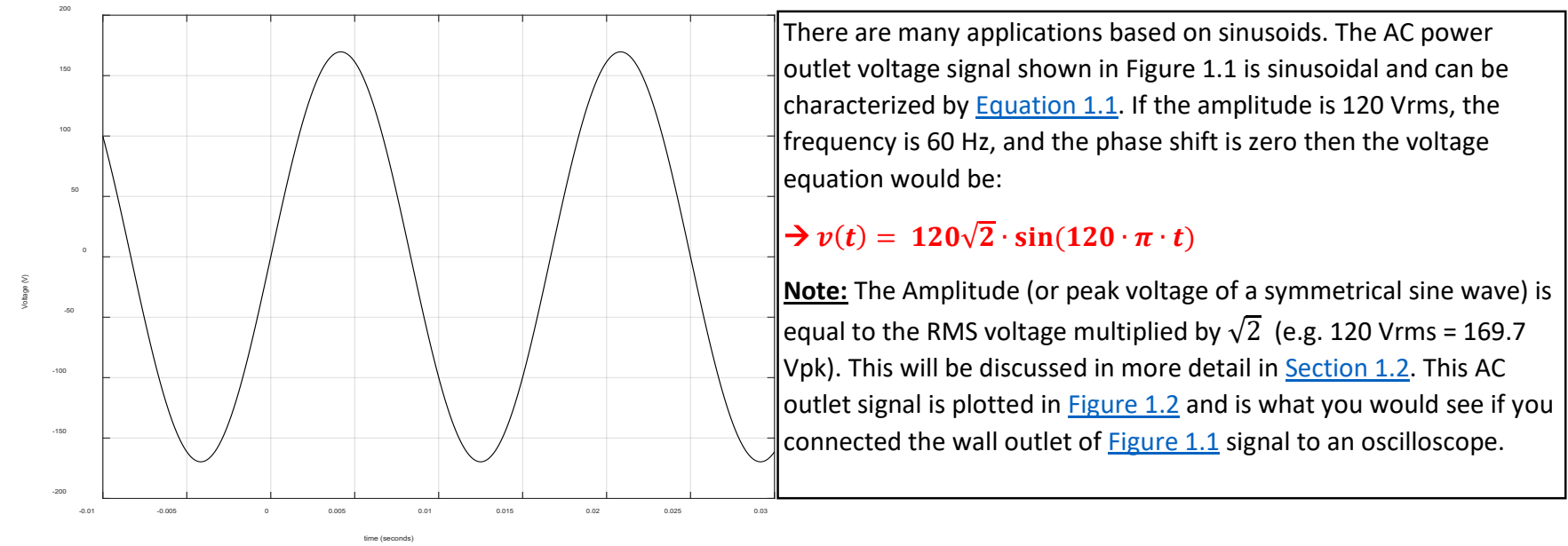

Figure 1.2: Standard AC outlet signal in U.S. homes (120 Vrms, $60 \mathrm{~Hz}$ sinusoid)

\section{Section 1.2 - Sinusoids}

A sinusoid is described in this eBook as a signal that is governed by Equation 1.1. A sine wave or a cosine wave are both considered sinusoids and they can be converted to each other through a $90^{\circ}$ (or a $\pi / 2$ radians) phase shift.

- $\sin (\omega t)=\cos (\omega t-\pi / 2) \quad$ or $\quad \cos (\omega t)=\sin (\omega t+\pi / 2)$ 
Equation 1.1 will be referred to as "sinusoidal form" throughout this eBook. Since it is desirable to have zero initial values for voltage and current sine waves are preferred for circuits (instead of cosine waves that have max amplitude at $\mathrm{t}=0$ ). The terms sinusoid and sine wave will often be used interchangeably in this eBook.

[1.1]

$$
v(t)=A \cdot \sin (\omega \cdot t+\theta) \text { or } i(t)=A \cdot \sin (\omega \cdot t+\theta)
$$

- $\quad \mathbf{A}=$ Amplitude (or peak value of the sinusoid assuming the signal is symmetric about the $\mathrm{x}$ axis)

- $\boldsymbol{\theta}=$ Phase shift (units = radians)

- $\mathbf{t}=$ time (units = seconds)

- $\omega=$ angular frequency (units $=\mathrm{rad} / \mathrm{sec}$ )

- $v(t)$ or $i(t)=$ The equation for the AC voltage or current. (The Amplitude sets the units of $v(t)$ or $i(t)$ )

$$
\omega=2 \cdot \pi \cdot f=\frac{2 \cdot \pi}{T} \quad \mathbf{f}=\text { Frequency (units = Hertz) and } \mathbf{T}=\text { Period (units = seconds) }
$$

In order to plot a sinusoid that is symmetrical about the x-axis (i.e. no DC offset) from the sinusoidal form (Equation 1.1) or to conversely write the sinusoidal form equation from the plot you need to know 3 things:

1) Amplitude (A): This can be measured from the difference of the maximum and minimum voltage divided by $2 \rightarrow \underline{A}=(\max -\min ) / 2$ If the sinusoid is symmetric then $A=\max =|\min |$, but if it is NOT symmetric then a DC offset is added to Equation 1.1 as follows: $v(t)=V_{D C}+A \cdot \sin (\omega \cdot t+\theta)$

2) Period (T): The period can be measured as the time between sinusoidal peaks on the plot. Once the period is measured, $\omega$ can be calculated as $\boldsymbol{\omega}=\mathbf{2} \cdot \boldsymbol{\pi} / \mathbf{T}$ as shown in Equation 1.2.

3) Time Shift $\left(t_{s}\right)$ : The time shift is determined by measuring the distance between the $y$ axis and the nearest starting cycle of the sine wave. If the sine wave begins its positive cycle before $x=0$ (left of the $y$ axis) then ts has a negative value and the sinusoid is said to be "advanced". If the sine wave begins its positive cycle after $x=0$ (right of the $y$ axis) then $t_{s}$ has a positive value and the sinusoid is said to be "delayed". Once the angular frequency $(\omega)$ and time shift $\left(t_{s}\right)$ are known, then the phase shift $(\theta)$ can be determined from $\left(-\boldsymbol{\omega} \cdot \mathbf{t}_{\mathbf{s}}\right)$ as shown in equations 1.3 . 
Example 1.1) Determine the sinusoidal form equation for the signal in Figure 1.2.

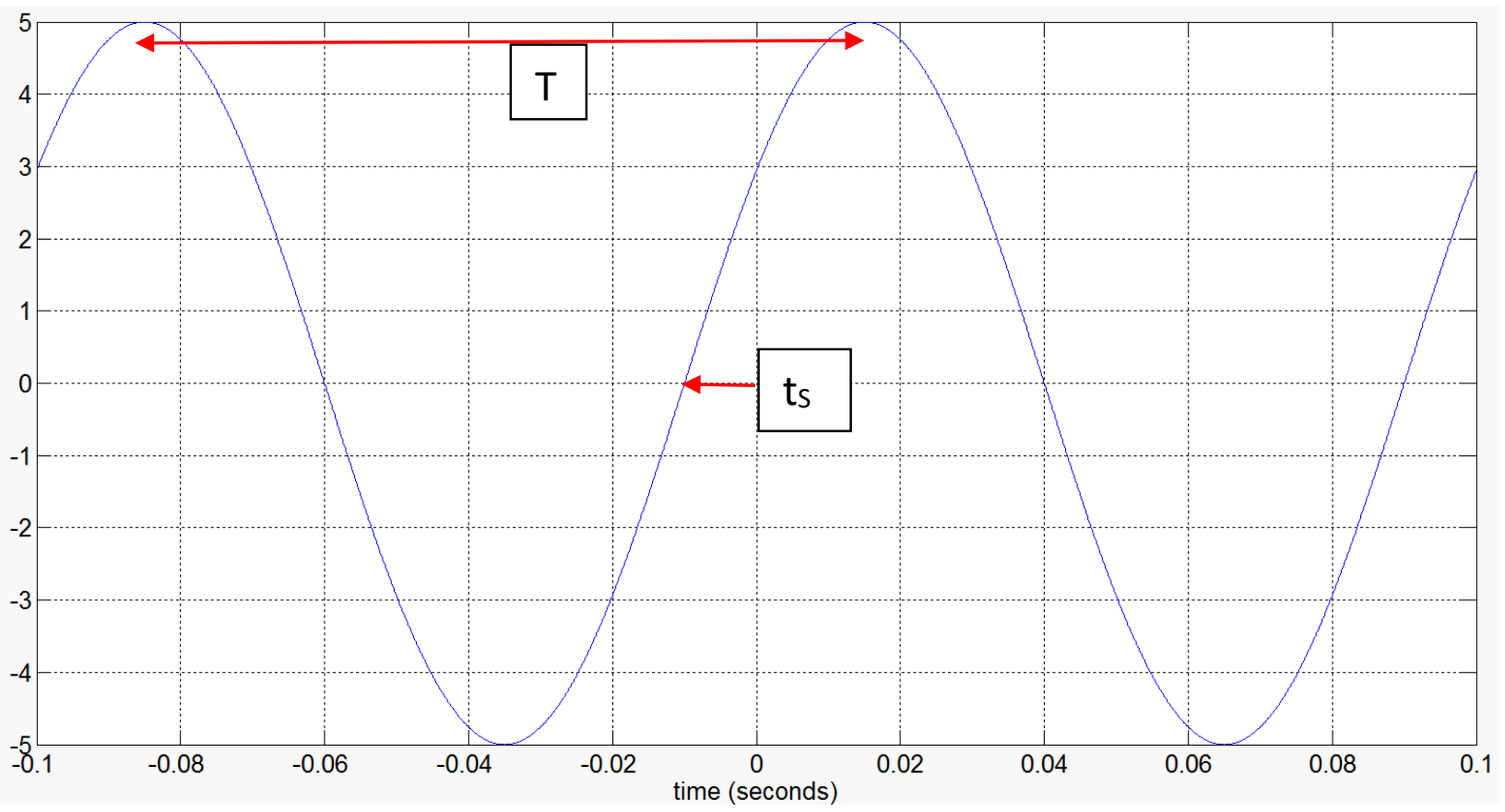

Figure 1.3: Plot of a symmetric sine wave used in Example 1.1.

Find the 3 items need to write the sinusoidal equation $\left(\underline{\mathbf{A}}, T, t_{s}\right)$ and convert T to $\underline{\boldsymbol{\omega}}$ and $t_{s}$ to $\underline{\boldsymbol{\theta}}$.

1) Amplitude $=\mathbf{A}=\underline{\mathbf{5}}$ (no units for the $y$ axis are given, but we would normally assume it is volts).

2) Period $=T=\sim(0.015$ seconds $)-(-0.085)=0.1 \mathrm{sec}$ or $100 \mathrm{msec}$. Once the period is measured, $\omega$ is calculated as $\omega=2 \cdot \pi / T=2 \cdot \pi / 0.1=\underline{\mathbf{2 0} \pi \mathrm{rad} / \mathrm{sec}}$

3) $t_{s}=$ the time shift is $\sim-0.01$ seconds as shown by the red arrow on Figure 1.1. Now that $t_{s}$ and $\theta$ are known the phase shift can be calculated by Equation $1.3 \rightarrow \theta=-\omega \cdot t_{s}=-20 \pi(-0.01)=\underline{\mathbf{0 . 2}} \boldsymbol{\pi}$ Using results from 1$)$ through 3 ) yields $\rightarrow v(t)=\mathbf{5} \sin (\mathbf{2 0} \boldsymbol{\pi} \cdot \mathrm{t}+\mathbf{0 . 2} \boldsymbol{\pi})$

Since the time shift was negative the sine wave in Figure 1.2 is considered to be "advanced" when compared to a sine wave with no phase shift.

Example 1.1 showed how to determine the sinusoidal form equation when a plot of the sine wave is available. Example 1.2 shows how to plot the sine wave if the equation is available.

Note: If you want to find the phase difference between two signals it is often easier to measure the time difference between the peaks and then use Equation 1.3 to convert to phase (see Figure 2.2 in Module 2).

Example 1.2) Plot the sine wave that is represented by the equation: $i(t)=20 \cdot \sin \left(200 \cdot t-30^{\circ}\right) \mathrm{mA}$

Note: In the equation for Example 1.2 the units are given at the end of the equation as $\mathrm{mA}$. Whenever you write the sinusoidal form of a voltage or current you should include the units at the end like this. If no units are given assume $v(t)$ is Volts and $i(t)$ is Amps. Additionally, the phase shift is listed with a degree sign, but the phase shift must be in radians for the units to agree. Since most people can comprehend degrees better than radians listing the phase shift in degrees is a common practice. If you do this make sure you label it with the degrees symbol.

CAUTION: If you are analyzing an equation that lists phase shift in degrees make sure you convert it to radians before adding it to the $\omega \cdot t$ term in the sinusoidal form equation. 
First, convert the phase shift to radians. $\underline{\theta=-30^{\circ} *}\left(\pi\right.$ radians $\left./ 180^{\circ}\right)=\underline{-\pi / 6 \text { radians }}$

To plot the sine wave from the sinusoidal form equation you need $A, T$, and $t_{s}$.

1) $\quad \mathbf{A}=\mathbf{2 0} \mathrm{mA}$ (Make sure the $y$ axis is labeled with $\mathrm{mA}$ )

2) $\underline{T=2 \pi / \omega}=2 \pi / 200=0.01 \pi=\underline{\mathbf{3 1 . 4 1 5 9} \mathbf{~ m s e c}}$

3) $\underline{t_{s}}=-\theta / \omega=-(-\pi / 6 \mathrm{rad}) /(200 \mathrm{rad} / \mathrm{sec})=\underline{\mathbf{+ 2}} .618 \mathrm{msec}$

Now that these three parameters are known the sine wave can easily be plotted by hand. Use $\mathbf{t}_{\mathbf{s}}$ to determine where to start the sine wave. Use $\mathbf{A}$ to know how far above and below the $\mathbf{X}$ axis it goes and use $\mathbf{T}$ to determine how much time elapses in one cycle of the sine wave. The final plot should look like the following figure. The Matlab code is shown to the right of Figure 1.4.

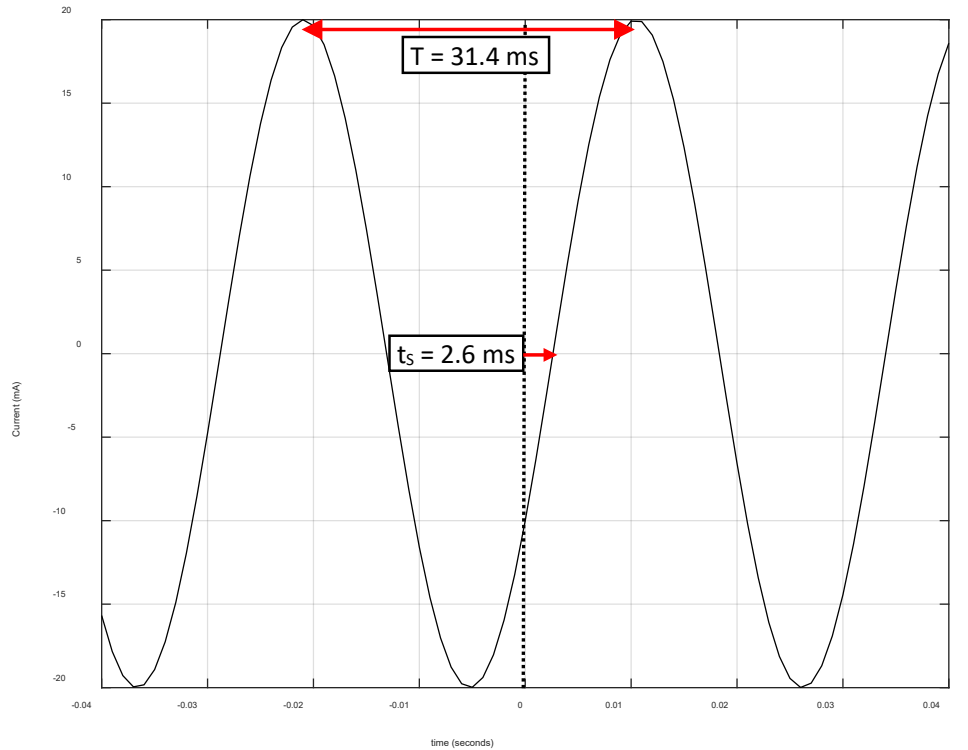

$\%$ Step 1 - Determine the Amplitude, A

$A=20 ; \%$ Make sure $m A$ is on y axis label

$\%$ Step 2 - Determine the Period, $T$

$\mathrm{w}=200 ; \%$ This is given in Example 1.2

$\mathrm{T}=2^{*} \mathrm{pi} / \mathrm{w} ; \%$ This is used to draw $\mathrm{i}(\mathrm{t})$ by hand

\% Step 3 - Determine the Time Shift, ts

Theta = -30; \% Degrees (This is Given in Example 1.2)

Theta $=-30 * \mathrm{pi} / 180 ; \%$ Radians

ts $=-$ Theta/ $\mathrm{w} \%$ This is used to draw $\mathrm{i}(\mathrm{t})$ by hand

$\%$ Plotting in Matlab

$\mathrm{t}=-.04: .001: .04$

$\mathrm{i}=\mathrm{A}^{*} \sin \left(\mathrm{w}^{*} \mathrm{t}+\right.$ Theta $)$;

$\operatorname{plot}\left(\mathrm{t}, \mathrm{i},{ }^{\prime} \mathrm{k}\right)$

grid on

xlabel('time (seconds)')

ylabel('Current (mA)')

Figure 1.4: Plot for Example 1.2

Note: In Module 3 we will use the term "lead" to describe a signal that is an advanced version of another signal and "lag" to describe a signal that is a delayed version of another signal.

For example, in Figure 1.5 the left plot shows that the solid black sine wave is advanced (or leading the dashed blue sine wave) because the time shift of the solid black sine wave (referenced to the blue sine wave) is a negative value. The following it the best way to think of this:

$>$ The leading sine wave is the sine wave that completes its sinusoidal cycle first.

The right plot of Figure 1.5 shows the opposite case where the solid black sine wave has a time shift that is positive resulting in a delay. In the right plot the black sine wave can be said to be lagging the blue dashed sine wave. Keep in mind you can also say it in the reverse order and change lagging to leading (i.e. the blue sine wave in the right plot of Figure 1.5 is leading the black sine wave because its sinusoidal cycle is completed first).

Some of the Matlab code for plots in this eBook are included at the end of the Appendix. 

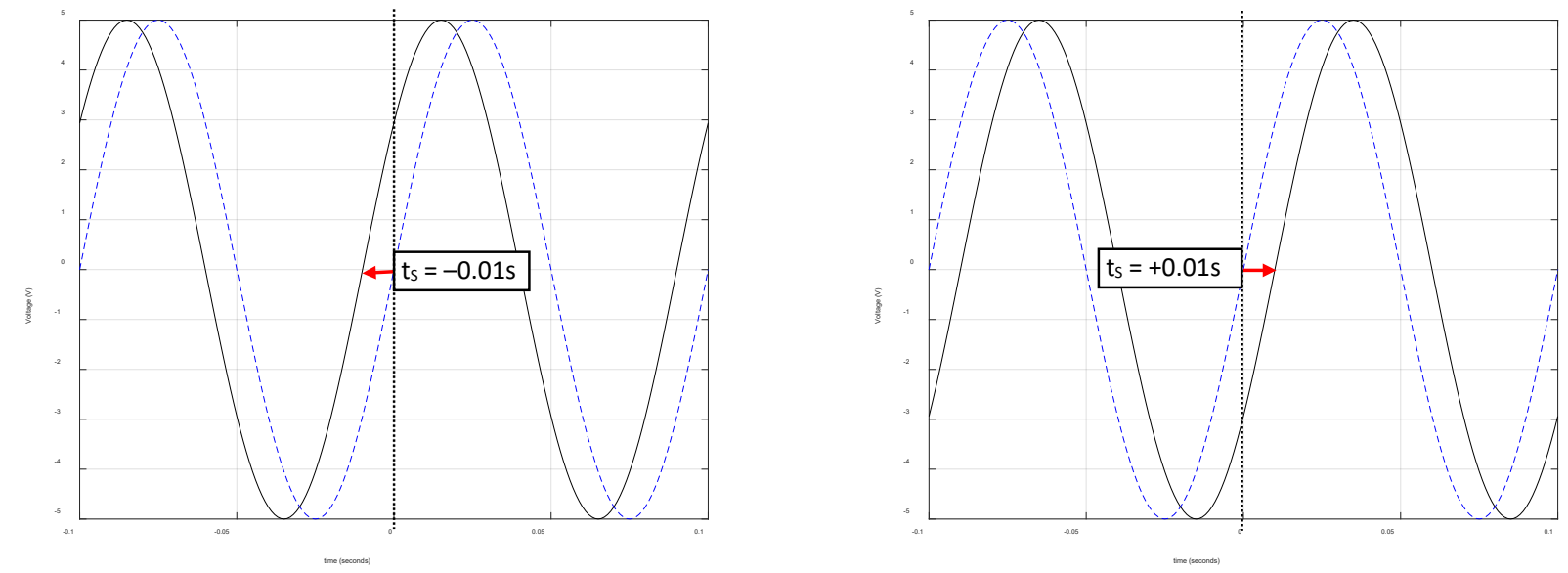

Figure 1.5: Dashed Blue Line (Zero time shift), Solid Black Line (Left, $t_{s}=-0.01 \mathrm{~s}$, Right, $\left.t_{s}=+0.01 \mathrm{~s}\right)$

Music is another area where sinusoids are present. Each musical note is a sinusoid at a different frequency. If you are new to the idea of frequency a good way to understand it is download a tone generator app that will play different frequencies through your mobile device. You will find that as you increase the frequency that you will stop being able to hear the sound as the frequency approaches or slightly exceeds $20 \mathrm{kHz}$. This is due to the limitation of the human ear. Other animals, such as dogs, can hear much higher frequencies. The following link describes the frequencies of the different notes of the musical scale in detail:

http://www.allaboutcircuits.com/textbook/alternating-current/chpt-1/ac-waveforms/

When musical notes (or any sound for that matter) are played and different frequencies are present at different times a frequency versus time plot can be used to characterize the "spectrum" of the sound over time. Two popular frequency versus time plots are the spectrogram and the power spectrum density (psd) plot.

Figure 1.6 shows the spectrogram and the psd for a train whistle sound that is sampled at a frequency of $8 \mathrm{kHz}$. See this link for more information about sampling.

The Spectrogram (top plot of Figure 1.6) shows the frequency versus time and uses a color code to show the amplitude at different frequencies. The psd plot (bottom plot of Figure 1.6) shows the amplitude versus normalized frequency (Actual_Frequency/0.5-Sampling_Frequency) for a block of time, which in this case is the complete 1.6 seconds of the train whistle sound. The 6 red lines of the spectrogram can be correlated to the 6 spikes in the psd plot. These plots help characterize sound so operations can be performed to enhance/amplify certain frequencies (e.g. an audio equalizer) or remove/attenuate certain frequencies (i.e. filtering - See Module 4). Another reason characterizing the frequency spectrum of a signal is important is that the sounds can be replaced by computer generated sounds. For example, vocoders (like Siri on an iPhone) digitally speaks like a human by pre-analyzing the spectrum of speech for the language setting that is selected. In Module 4 another type of frequency response plot called the Bode plot will be used to characterize RLC filters. 

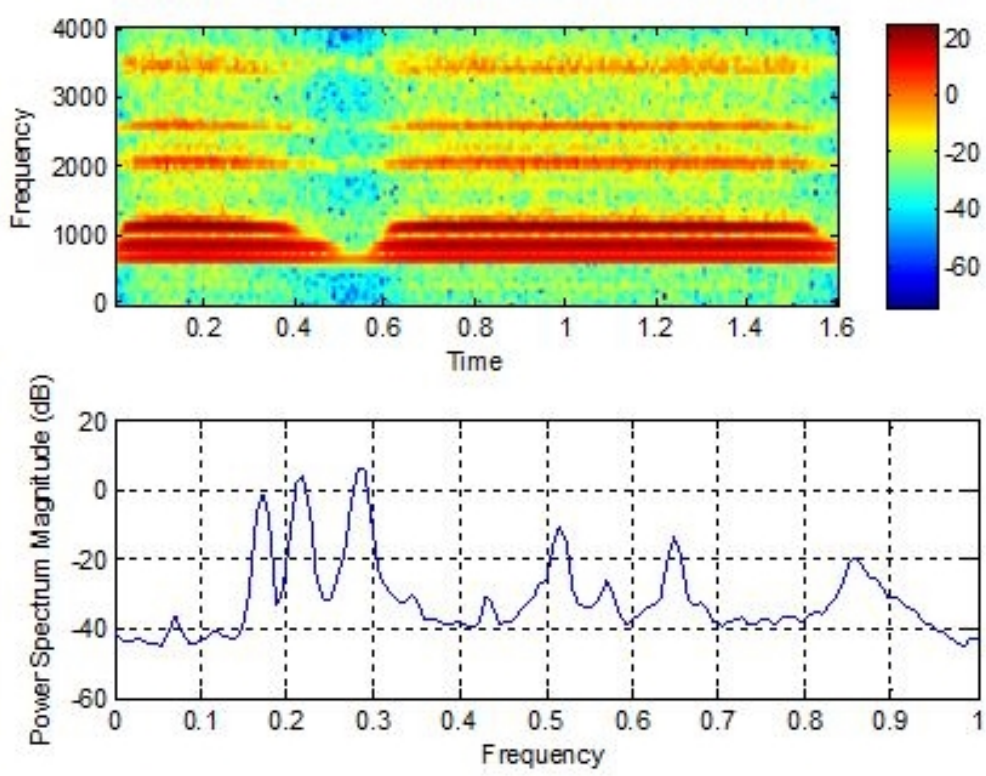

Figure 1.6: Spectrogram (top) and psd plot (bottom) of a train whistle sound

The following statement is perhaps the most important thing about sinusoids.

\section{Any periodic waveform (one that repeats every $T$ seconds) can be created by a sum of sinusoids.}

For example, a square wave with amplitude $(A)$ and a fundamental frequency $\left(f_{0}\right)$ is created by taking an infinite sum of odd harmonic sine waves as follows (The red bolded numbers are called the harmonics):

$v(t)=\frac{4 \cdot A}{\pi}\left[\frac{\sin \left(1 \cdot 2 \pi f_{0} \cdot t\right)}{1}+\frac{\sin \left(3 \cdot 2 \pi f_{0} \cdot t\right)}{3}+\frac{\sin \left(5 \cdot 2 \pi f_{0} \cdot t\right)}{5}+\frac{\sin \left(7 \cdot 2 \pi f_{0} \cdot t\right)}{7}+\cdots+\frac{\sin \left(N \cdot 2 \pi f_{0} \cdot t\right)}{N}\right]$

\section{http://www.allaboutcircuits.com/textbook/alternating-current/chpt-7/square-wave-signals/}

A triangle wave with amplitude $(A)$ and fundamental frequency $\left(f_{0}\right)$ is also created by taking an infinite sum of odd harmonic sine waves, but with slightly different constants and alternating signs of each term:

$v(t)=\frac{8 \cdot A}{\pi^{2}}\left[\frac{\sin \left(1 \cdot 2 \pi f_{0} \cdot t\right)}{1}-\frac{\sin \left(3 \cdot 2 \pi f_{0} \cdot t\right)}{9}+\frac{\sin \left(5 \cdot 2 \pi f_{0} \cdot t\right)}{25}-\frac{\sin \left(7 \cdot 2 \pi f_{0} \cdot t\right)}{49}+\cdots-\frac{\sin \left(N \cdot 2 \pi f_{0} \cdot t\right)}{N^{2}}\right]$

These links from Wikipedia show the equations and also have an option for you to play the sound of the waveforms.

https://en.wikipedia.org/wiki/Square wave https://en.wikipedia.org/wiki/Triangle wave

The square and triangle waves will be closer to exact as more terms (or harmonics) are included. Figure 1.7 (see Matlab code in the Appendix) shows a square and triangle wave created with different numbers of terms with $A=1$ and $f_{0}=60 \mathrm{~Hz}$. 
10 sinusoidal terms

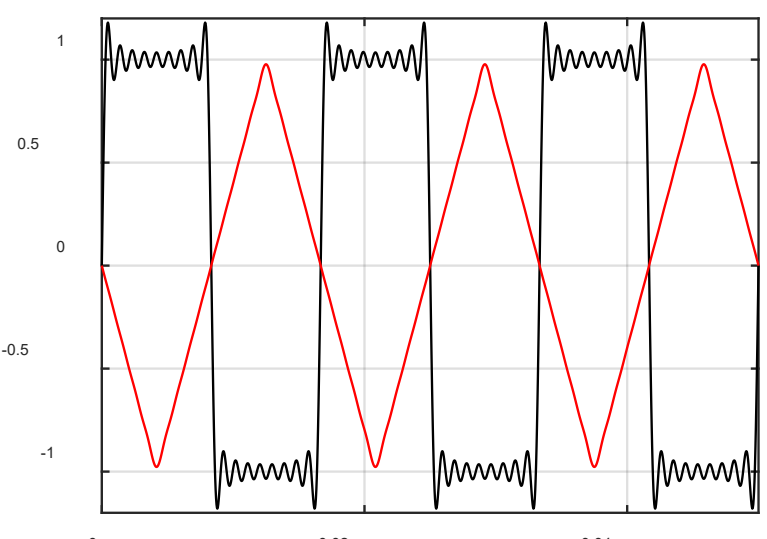

0.04

100 sinusoidal terms

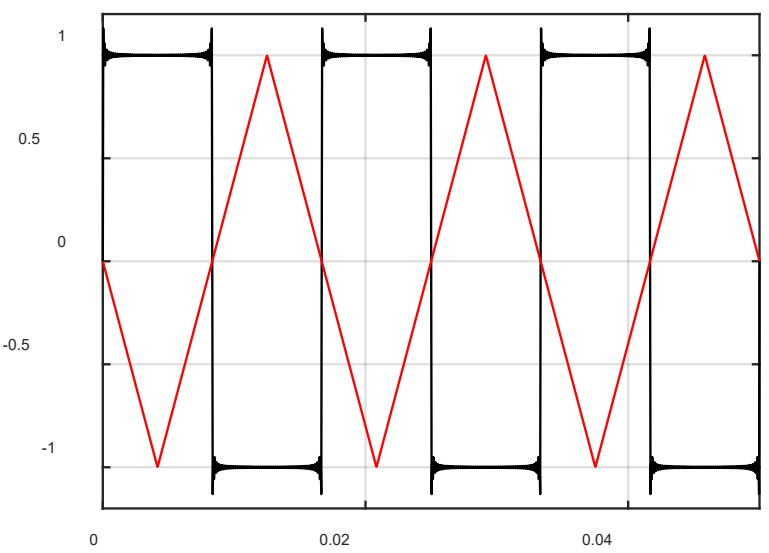

20 sinusoidal terms

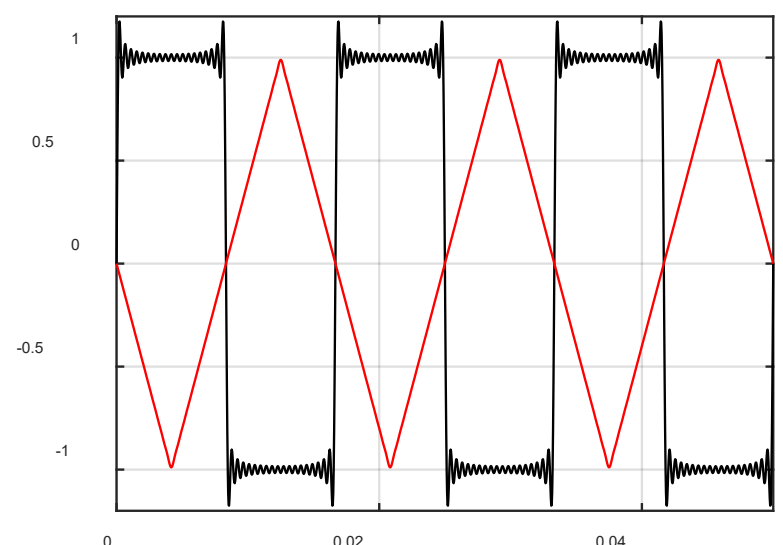

1000 sinusoidal terms

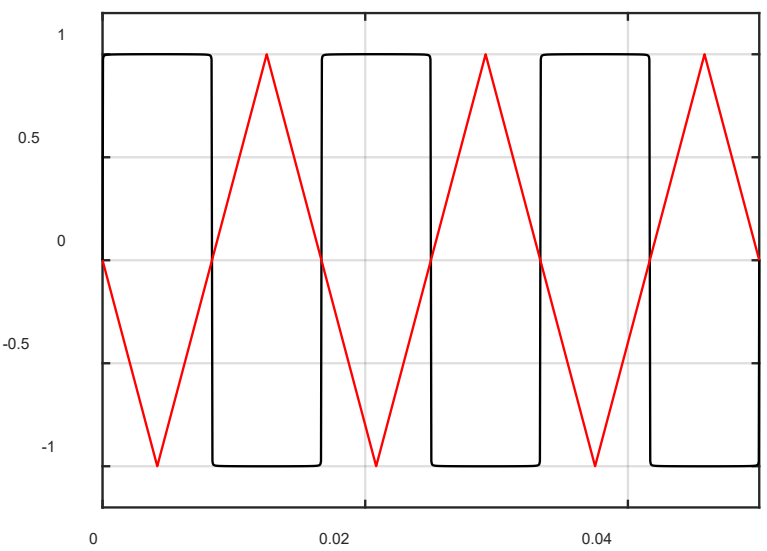

Figure 1.7: Square wave (black) and Triangle wave (red) created with different numbers of sinusoidal terms.

\section{Section 1.3- Defining AC Voltage Types for Common AC Waveforms}

In this section the three common waveforms that have been previously discussed (sine wave, square wave, and triangle wave) will be described in more detail. These three are the most common waveforms and are the three that are available in practically all function generators as shown in Figure 1.8 and Figure 1.9.

\section{ONLY periodic waveforms (signals that repeat their pattern every $T$ seconds) are discussed in this eBook.}

Figure 1.8 shows the Multisim tool that is a replica of the Agilent 33120A function generator. The knobs and buttons work in the same way as the actual Agilent device works. A red box is drawn around the sine wave, square wave, and triangle wave buttons. The Multisim symbol for the function generator has the title XFG1 and the output and ground connectors are wired into channel $A$ of the generic Multisim 2-channel scope that is titled XSC1. In Figure 1.8 a $1 \mathrm{kHz}$ square wave is selected. 


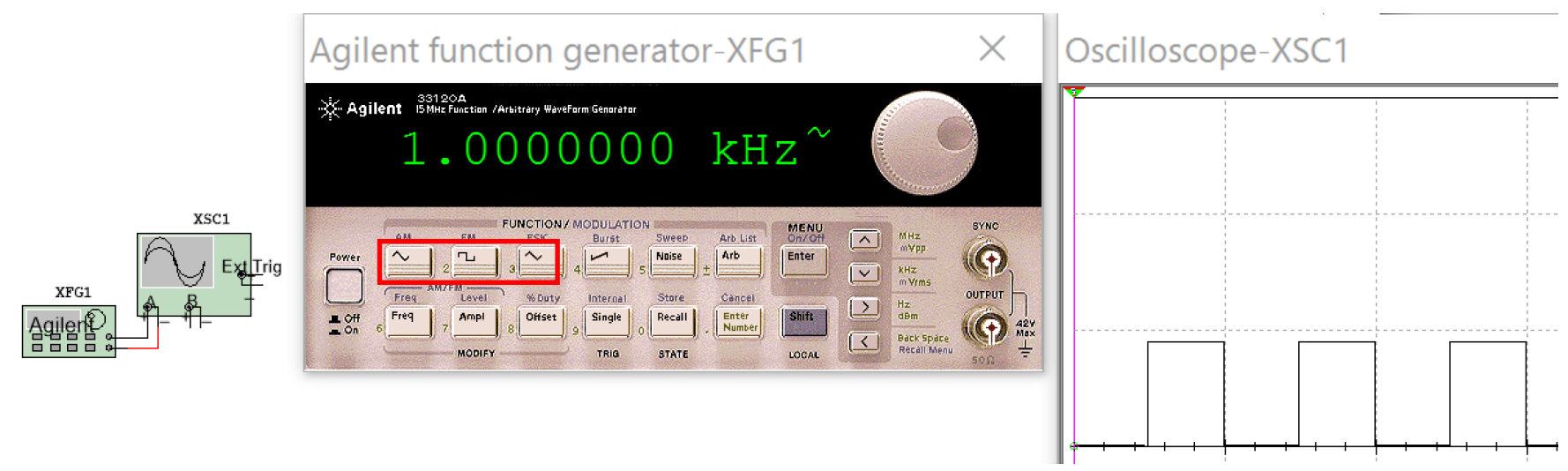

Figure 1.8: Agilent Function Generator and Multisim 2-Channel Oscilloscope.

Figure 1.9 shows the generic Multisim function generator tool and the scope tool that is a replica of the Tektronix TDS 2024 Oscilloscope. The knobs and buttons work in the same way as the actual Tektronix device works. The Multisim symbol for the function generator has the title XFG2 and the + output and ground connectors are wired into channel 2 of the Tektronix scope that is titled XSC1. In this example a $60 \mathrm{~Hz}$ sine wave with a peak voltage of $10 \mathrm{~V}$ is selected. The right side of the Oscilloscope shows 5 measurements. Figure 1.10 shows a zoomed in view of these 5 measurements.

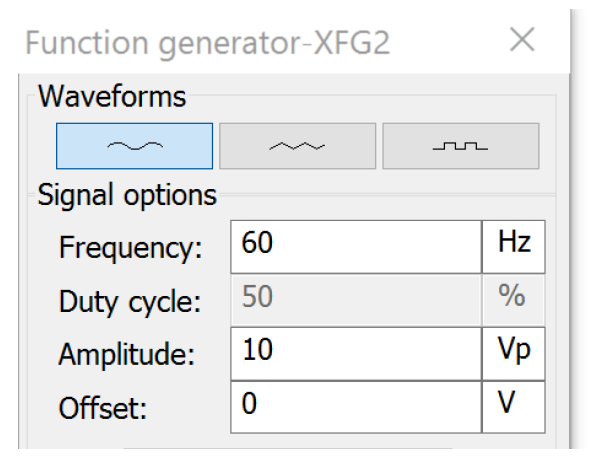

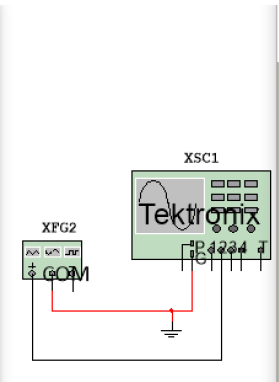

Figure 1.9: Multisim Function Generator and Tektronix Oscilloscope.

The primary goal of this section is to show how to solve the peak to peak (Vpp), peak (Vpk), average (Vavg), and root mean square voltage (Vrms) for the sine wave, square wave, and triangle wave. Figure 1.8 and Figure 1.9 shows two ways that you can simulate these three signals and two ways that you can make these four measurements in Multisim. Figure 1.10 shows that Vpk is called Vmax and the Vavg is called Vmean on the Tektronix oscilloscope. Another terminology note is that Vrms is frequently referred to as VAC. 


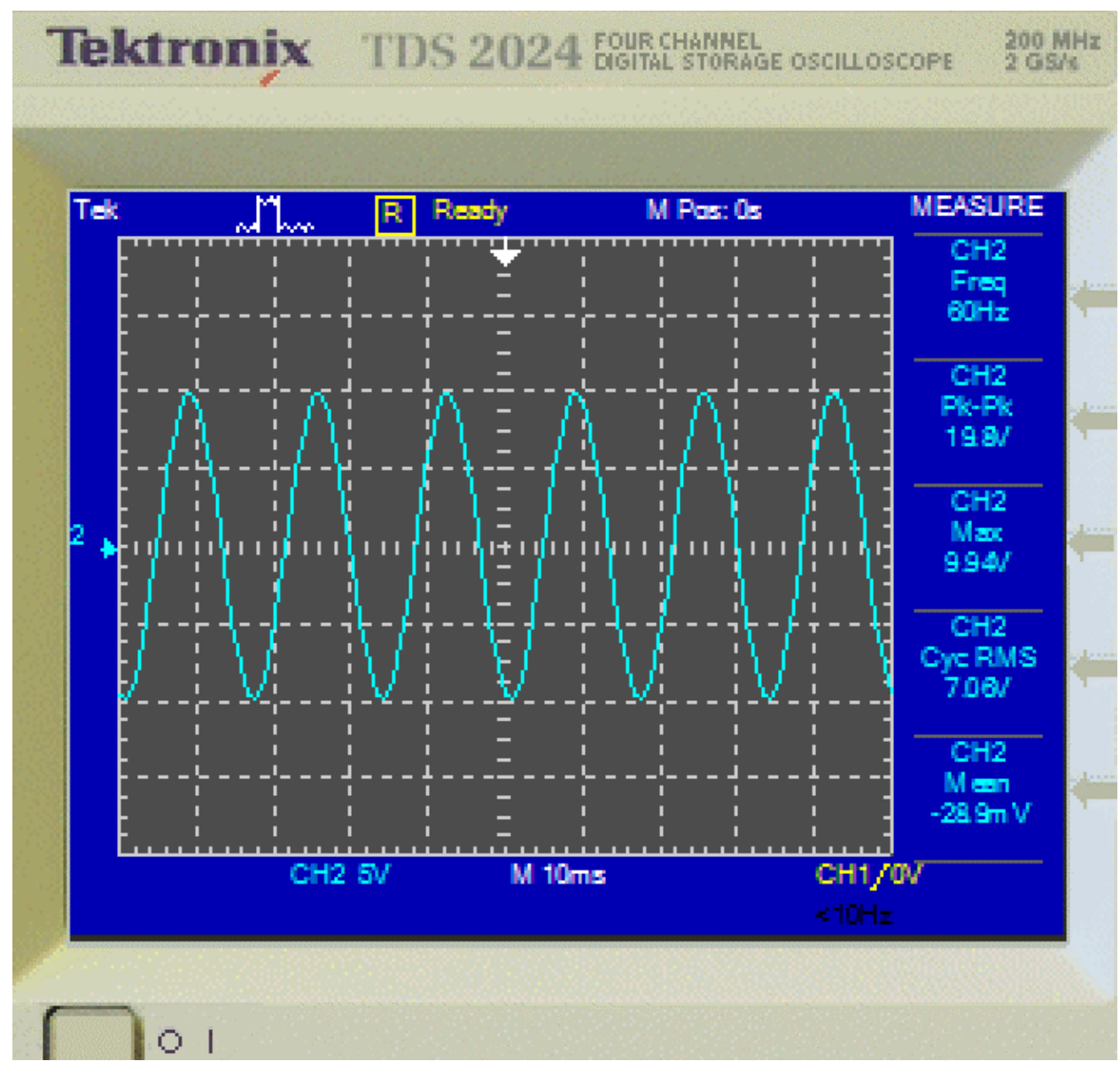

Figure 1.10: From top to bottom (F, Vp-p, Vpk, Vrms, and Vavg) Measurements from Figure 1.9

You can also use a Multimeter to measure AC waveforms, but you only get the RMS current or voltage. The meter in Figure 1.11 is put in AC mode (the wavy line) and is measuring the RMS current in the AC circuit. The voltage could also be measured if the meter was placed across a component and the $\mathrm{V}$ button selected.

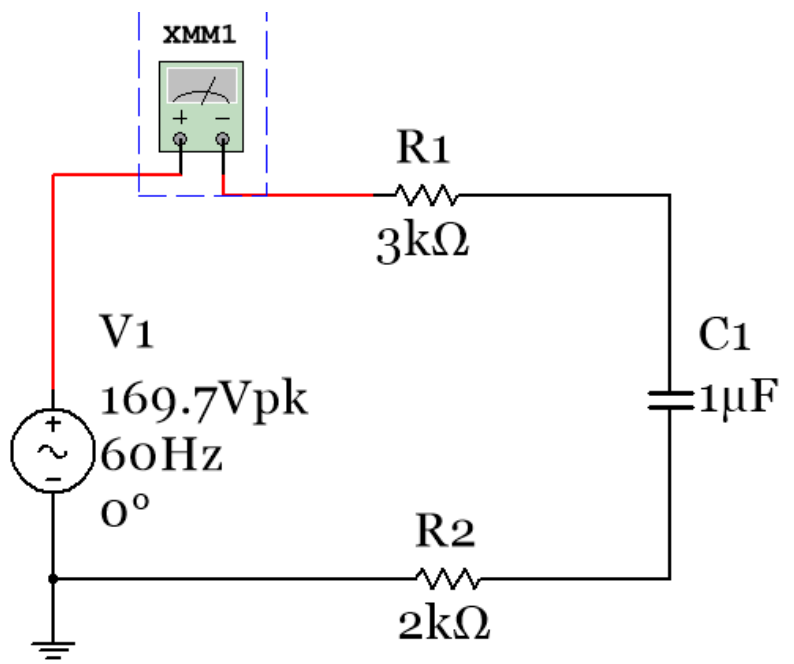

\section{Multimeter-XMM1}
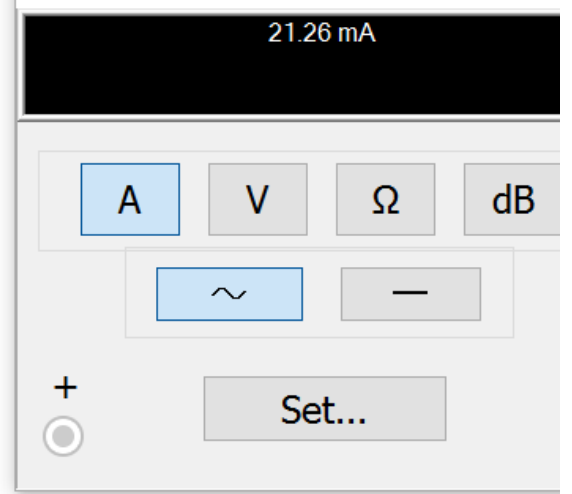

Figure 1.11: Using the Multsim Multimeter to measure the RMS current (also called IAC) 


\section{Section 1.3.1 - Peak and Peak to Peak Voltage}

Determining the peak (Vpk) and peak to peak (Vpp) voltage (or current) for the sine wave, square wave, and triangle wave is a simple process, as described in the two bullets below. The voltage variable will primarily be used in this eBook, but the following AC currents can also be determined: Ipp, Ipk, Irms, and lavg

- Vpk is the maximum value of any periodic waveform. This includes sine, square, and triangle waves.

- Vpp is the difference between the maximum and minimum values of a periodic waveform.

- A, which is the amplitude of a sinusoid that was defined in section 1.2, is equal to $\mathrm{Vpp} / 2$. It is also equal to Vpk if the sinusoid is symmetric about the $x$-axis (i.e. There is no DC offset and $\max =|\min |$ ).

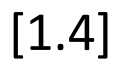

$$
A=\frac{v_{p p}}{2}
$$

Where: $\boldsymbol{v}_{\boldsymbol{p} \boldsymbol{p}}$ is the maximum - minimum of a sinusoid

Figure 1.12 shows both scenarios: with and without a DC offset. Keep in mind that when measuring an AC signal on an oscilloscope you must put it in DC coupling mode if you want to see the DC offset. When the oscilloscope is in AC coupling mode the DC offset is automatically filtered out. The DC coupling setting is changed by clicking on the Blue shaded button that corresponds to the channel on the bottom of the oscilloscope (Figure 1.12 shows both channels are in DC coupling mode). This figure shows that XFG1 has a symmetric (DC offset $=0$ ) triangle wave with a Vpp of $16 \mathrm{~V}$ (amplitude $=8$ ) and sine wave with a $5 \mathrm{~V} \mathrm{DC}$ offset and a Vpp of $20 \mathrm{~V}$ (Amplitude =10).

Note: To change the color of the plot in the Multisim oscilloscope you have to change the wire color going into the + terminal on channel A or B. To change the color you right click on the wire and click on "segment color". Also, if the - pin of the oscilloscope is desired to be referenced to ground you can leave it unwired.

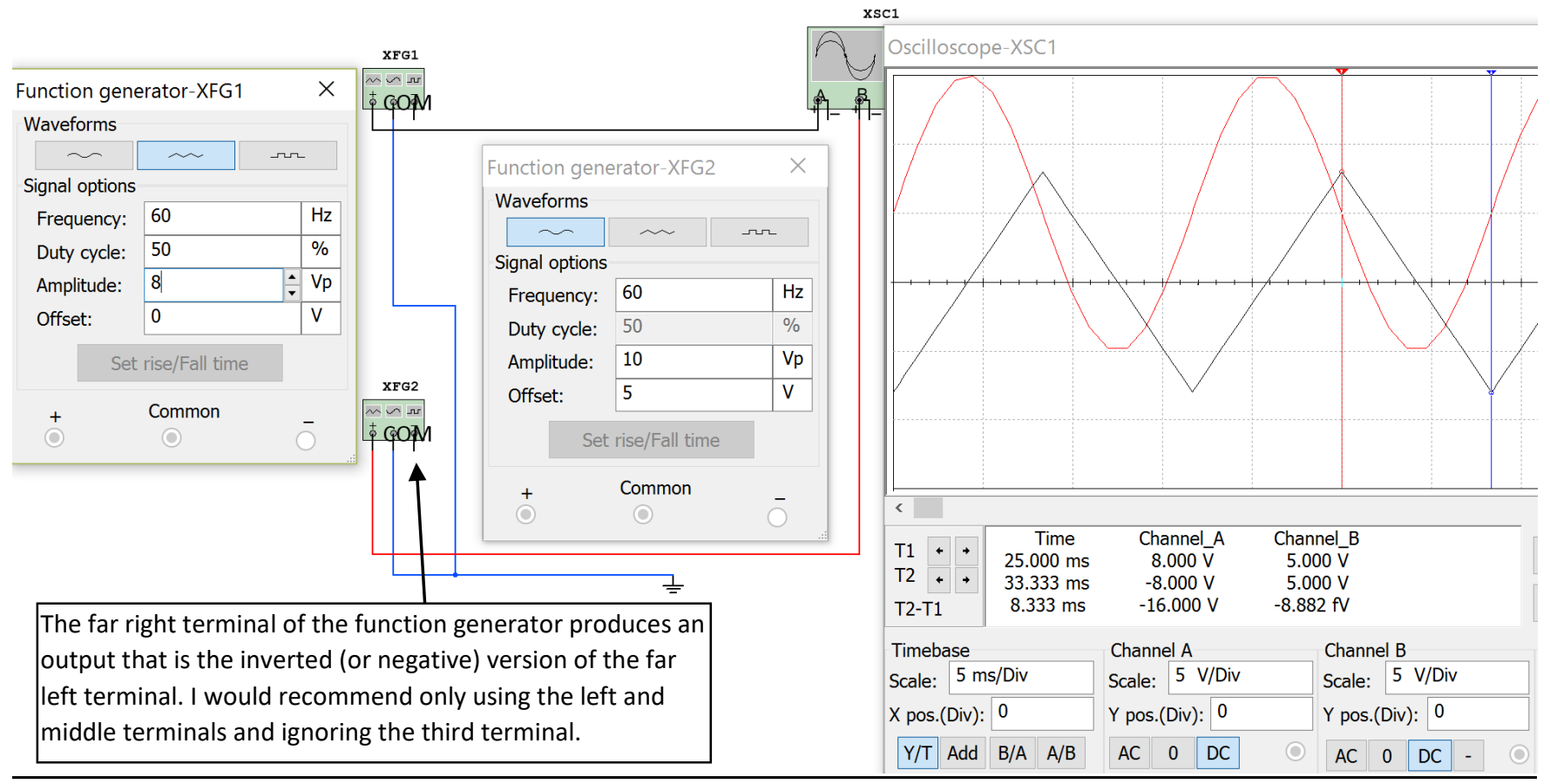

Figure 1.12: Using the Multisim function generator to plot two waveforms on the Multisim oscilloscope 


\section{Section 1.3.2 - Average Voltage}

The average voltage (Vavg) is the voltage measured by a Multimeter in DC mode. Periodic signals (i.e. they repeat their pattern every $T$ seconds) that are symmetrical about the $x$-axis have an average voltage of zero. The average value for a waveform that is not symmetric can either be calculated by using integration (Equation $\underline{1.5}$ ) or using the area method. The area method is preferred for most waveforms because it is usually much easier to calculate. This is especially true for square waves and triangle waves.

$$
\text { [1.5] } \quad v_{\text {avg }}=\frac{1}{T} \int_{0}^{T} v(t) d t
$$

Where: $\boldsymbol{v}(\boldsymbol{t})$ is the periodic signal, $\boldsymbol{T}$ is the period

The average value of any periodic signal can also be solved numerically using the area method.

Step 1) Break up the periodic square wave into rectangles over 1 period and label the areas $A_{l,} A_{I I}, A_{I I I}, A_{I V}$, etc. Step 2) Find the sum of the areas over 1 period $\left(A_{\text {Total }}\right.$. Note: Negative voltage peaks produce negative areas. Step 3) Divide the sum of the area $\left(A_{\text {Total }}\right)$ by the period $(T) \rightarrow v_{\text {avg }}=A_{\text {Total }} / T$

\section{Section 1.3.3 - Root Mean Square (RMS) Voltage}

Vpk, Vpp, and Vavg are key AC voltage quantities, but the most important AC voltage type is the RMS voltage (Vrms). The Root Mean $\underline{\text { Square }}$ (RMS) voltage is found by:

1) Squaring all (or as many points as possible) along one period of a periodic waveform.

2) Finding the Mean (or the average) of all of the square values found in step 1).

3) Taking the square Root of the mean found in step 2).

The RMS value for any periodic waveform can be calculated mathematically by using Equation 1.6.

$$
v_{r m s}=\sqrt{\frac{1}{T} \int_{0}^{T} v(t)^{2} d t}
$$

Where: $\boldsymbol{v}(\boldsymbol{t})$ is the periodic signal, $\boldsymbol{T}$ is the period

The RMS voltage is an important quantity in AC circuits because it allows the AC power delivered to a load to be equated to the DC power. Vrms can be defined as the magnitude of AC voltage that produces the same amount of average power on a load as an equivalent DC voltage, as shown in Figure 1.13.
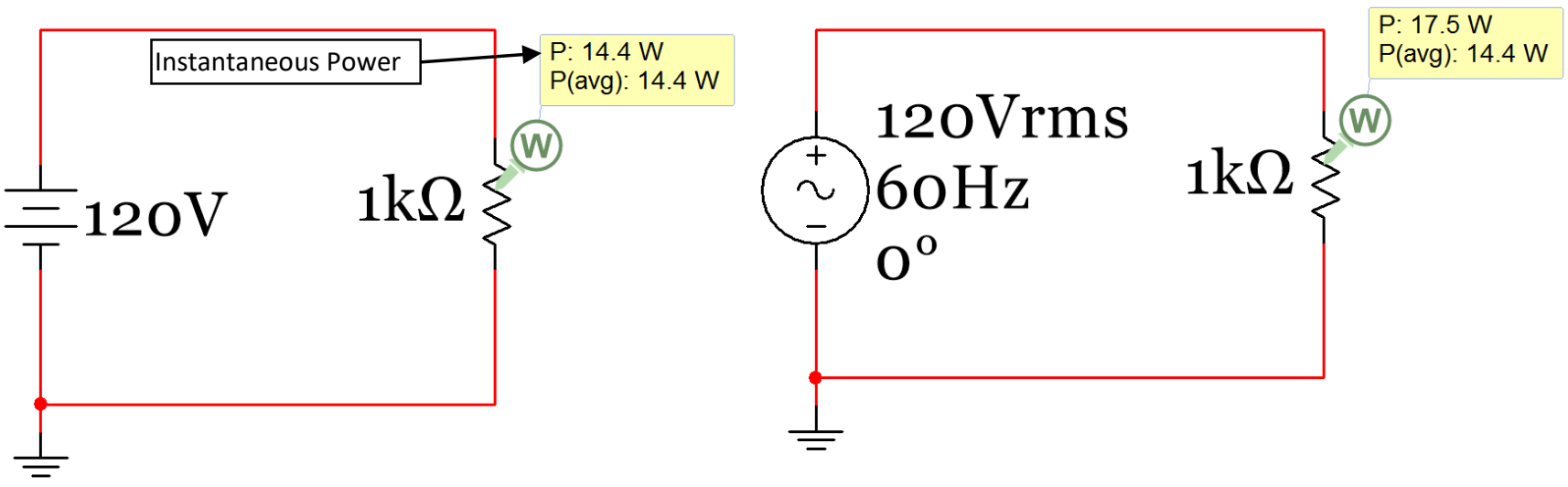

Figure 1.13: Instantaneous and Average Power of a circuit with a DC source (left) and an AC source (right). 
The top power measurement in the power probe box in Figure 1.13 is the "instantaneous power" ( $P_{\text {inst }}$ ) and the bottom power value is called the "average power" $\left(P_{A v G}\right)$. Since voltage and current doesn't change in a DC circuit at steady state $P_{\text {inst }}$ and $P_{\text {avg }}$ are the same. For $A C$ circuits $P_{\text {inst }}$ is determined in the same way as it is in a DC circuit, but $P_{\text {avg }}$ is determined using RMS current and voltage. The $P_{\text {inst }}$ value continually changes in an AC circuit as the voltage and current change. At the moment in time the MultiSim circuit in Figure 1.13 was paused, $P_{\text {inst }}$ just happened to be equal to $17.5 \mathrm{~W}$, but the voltage could theoretically have been anywhere from -Vpk to + Vpk depending on when the circuit was paused. Since $P_{\text {inst }}$ depends on the instantaneous voltage (or the exact voltage at the moment it was paused) it changes from 0 to $\mathrm{Vpk}^{2} / \mathrm{R}$. Recall in section 1.1 it was stated "it is a good idea to think of a DC signal as one that can be obtained by freezing time in an AC signal and looking at the instantaneous voltage or current values at that specified time."

Note: In the DC Circuits book instantaneous power $\left(P_{\text {inst }}\right)$ is given a positive value when it is dissipated (or absorbed) by a resistor, but a negative value when power is supplied (or delivered) by a source.

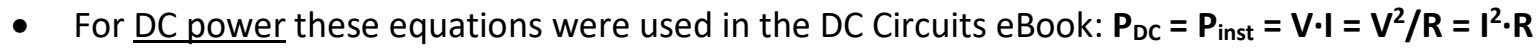

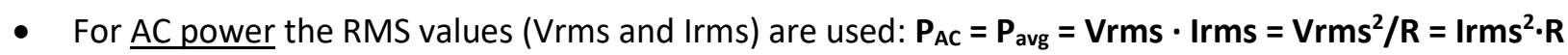

Caution: Make sure the RMS voltage (Vrms) and current (Irms) are used when AC power is calculated.

There are simplified methods for determining the RMS voltage or current for symmetrical waveforms (i.e. DC offset $=0$ ) as shown in equations 1.7 to 1.9 below. Notice only the peak voltage is needed to determine Vrms. The phase and frequency have no effect.

[1.7] $v_{r m s}=\frac{v_{p k}}{\sqrt{2}}=0.7071 v_{p k}$ Where: $\boldsymbol{V p k}$ is the peak of a symmetrical sinusoid

[1.8] $v_{r m s}=1 v_{p k}$ Where: Vpk is the peak of a symmetrical square wave

[1.9] $v_{r m s}=0.577 v_{p k}$ Where: $\boldsymbol{V p k}$ is the peak of a symmetrical triangle wave

For any periodic square waves (symmetrical or not symmetrical) the RMS value can be calculated using the following steps. This method will be referred to as the "modified area method" because it is a slight modification of the area method that was used to find the average voltage. If you get confused on the order of the steps, double checking the units can help catch mistakes.

Step 1) Break up the periodic square wave into rectangular sections over 1 period and square the peak voltage of each. If the section is above the $x$-axis square the maximum voltage and if it is below the $x$-axis square the minimum voltage. Call these values $\alpha_{1}, \alpha_{2}$, etc. The units are $V^{2}$ (or $A^{2}$ if it is a current waveform).

Step 2) Multiply each $\alpha$ value (found in Step 1) by the base of the rectangle ( $\Delta t$ ) so that you get $\alpha_{1} \cdot \Delta t_{1}, \alpha_{2} \cdot \Delta t_{2}$, etc. The units for each of these terms will be $V^{2} \cdot \sec$ (or $A^{2} \cdot \sec$ if it is a current waveform).

Step 3) Sum the values from Step 2 to get $\alpha_{\text {Total. }}$ The units will be $V^{2} \cdot s e c$.

Step 4) Divide the value of $\alpha_{\text {Total }}$ (determined in Step 3) by the period (T). The units will now be $V^{2}$.

Step 5) Take the square root of the result of Step 4. The units will now be $V$ and this result is Vrms.

Make sure you only use the modified area method for periodic square waves.

- Triangle waves and all other periodic waveforms (except only symmetrical sinusoids and periodic square waves) must use the integration method (Equation 1.6) to determine the RMS value. 


\section{Section 1.4- Sine Wave, Square Wave, and Triangle Wave Example Problems}

This section provides example calculations of Vpk, Vpp, Vavg, and Vrms for sine waves, square waves, and triangle waves. To save time and reduce calculation errors the integration formulas (Equations 1.5 and 1.6) should be avoided for situations where there is a simplified method. Table 1.1 shows the simplified methods available for different types of AC waveforms.

\section{Table 1.1) Table Showing Vpk and Vpp values and Simplified Methods for Determining Vavg and Vrms}

\begin{tabular}{|c|c|c|c|c|c|c|}
\hline & \multicolumn{3}{|c|}{ Waveforms that are symmetrical about $\mathrm{x}$-axis } & \multicolumn{3}{|c|}{ Waveforms that are NOT symmetrical } \\
\hline & Sine Wave & Square Wave & Triangle Wave & Sine Wave & Square Wave & Triangle Wave \\
\hline Vpp & \multicolumn{6}{|c|}{ Vpp is always the maximum voltage - the minimum voltage (i.e. Vmax-Vmin) } \\
\hline Vpk & \multicolumn{6}{|c|}{ Vpk is always the maximum voltage (i.e. Vmax) } \\
\hline Vavg & \multicolumn{3}{|c|}{0} & The DC offset & \multicolumn{2}{|c|}{ Area Method } \\
\hline Vrms & $\begin{array}{c}0.7071 \cdot \text { Vpk } \\
\text { Equation [1.7] }\end{array}$ & $\begin{array}{c}1.0 \cdot \text { Vpk } \\
\text { Equation [1.8] }\end{array}$ & $\begin{array}{c}0.577 \cdot \text { Vpk } \\
\text { Equation [1.9] }\end{array}$ & $\begin{array}{l}\text { Use Integration } \\
\text { Equation [1.6] }\end{array}$ & $\begin{array}{c}\text { Modified } \\
\text { Area Method }\end{array}$ & $\begin{array}{c}\text { Use Integration } \\
\text { Equation [1.6] }\end{array}$ \\
\hline
\end{tabular}

Section 1.4.1 - Example Calculations of Vpp, Vpk, Vavg, and Vrms for Sine Waves

Example 1.3) Using Table 1.1 as guidance determine Vpp, Vpk, Vavg, Vrms, and Amplitude of a symmetrical sine wave with the sinusoidal form equation $v(t)=6 \sin (200 t)$

- $\quad \mathrm{Vpp}=\max -(\min )=6-(-6)=\underline{\mathbf{1 2} \mathbf{V}}$ (measured to be $11.91 \mathrm{~V}$ with the Multisim oscilloscope and $11.9 \mathrm{~V}$ with the Multisim voltage probe). The true value is $12 \mathrm{~V}$ (which is twice the amplitude entered in XFG1).

- $\quad \mathrm{Vpk}=\max =\underline{\mathbf{6 V}}$ (measured as $5.958 \mathrm{~V}$ with the scope)

- Amplitude $=\mathrm{A}=\mathrm{Vpp} / 2=\underline{\mathbf{6 V}}$

- $\quad \operatorname{Vavg}=\underline{\mathbf{0 V}}$ (as stated in Table 1.1 for any symmetrical sine wave). The probe measures it as $2.24 \mathrm{mV}$.

- $V r m s=V p k / s q r t(2)=6 / s q r t(2)=\underline{4.24 V}$ (Table 1.1 says to use Equation 1.5 for symmetrical sine waves)

These values are verified below using the Multisim function generator, Multimeters, probes, and the scope.

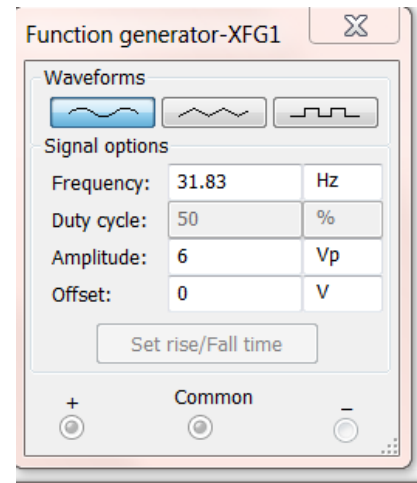

Agilent multimeter-XMM2
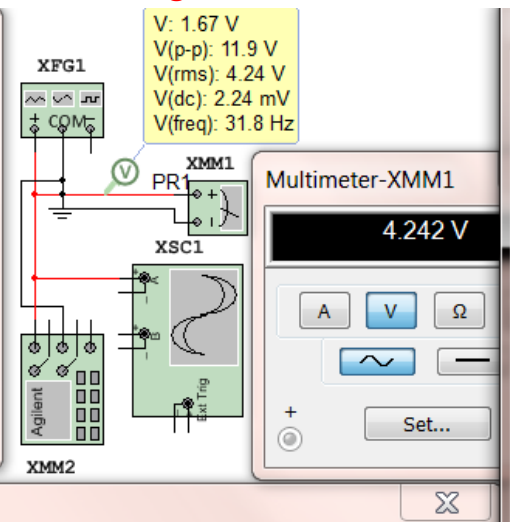

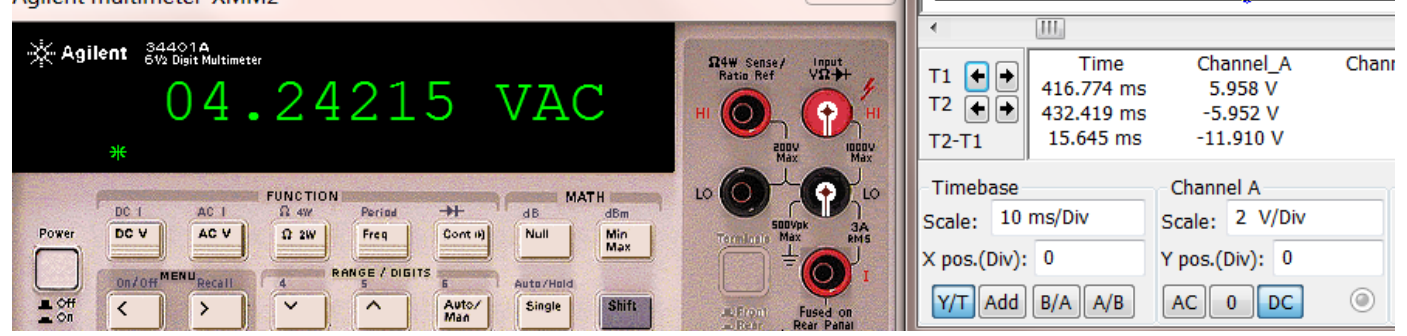

Figure 1.14: Function generator (XFG1), Multimeters (XMM1 and XMM2), Scope (XSC1), and Probe (box) 


\section{V: $1.67 \mathrm{~V}$ \\ $V(p-p): 11.9 \mathrm{~V}$ \\ $V(\mathrm{rms}): 4.24 \mathrm{~V}$ \\ $\mathrm{V}(\mathrm{dc}): 2.24 \mathrm{mV}$ \\ $V$ (freq): $31.8 \mathrm{~Hz}$}

\section{Figure 1.15: Close up view of the AC Voltage Probe from Example 1.3}

In the Multisim probe box from Example 1.3 (Figure 1.15) the Vpp, Vrms, Vavg, and the frequency are shown as $\mathrm{V}(\mathrm{p}-\mathrm{p})=11.9 \mathrm{~V}, \mathrm{~V}(\mathrm{rms})=4.24 \mathrm{~V}, \mathrm{~V}(\mathrm{dc})=2.24 \mathrm{mV}$, and $\mathrm{V}($ freq $)=31.8 \mathrm{~Hz}$ respectively. The actual peak to peak voltage (Vpp) should have been $12 \mathrm{~V}$, but the Multisim probes will often have a slight error as it does in this case. The Vavg (or $\mathrm{V}(\mathrm{dc})$ ) should have been 0 , but it reads $2.24 \mathrm{mV}$ in the probe box. Again, this level of inaccuracy can be expected. The top value that reads $1.67 \mathrm{~V}$ is called the "instantaneous voltage". For an AC signal this value constantly changes. If you freeze the time in an AC circuit the problem can be treated as a DC problem and the instantaneous voltage measured would be the DC voltage that would be used for that problem.

For example 1.3 the rms voltage probe and the two Multimeters all had the same result, but for the next example that has a sine wave with a DC offset it will show the two Multimeters do not produce the correct result. The DC offset cannot be accounted for with either of the Multisim Multimeter or the Agilent Multimeter because they have "AC Coupling" on the input, which removes DC before the measurement is made. Therefore, if you have a signal that is not symmetric only the voltage probe to can be used measure Vrms or Irms.

Example 1.4) Using Table 1.1 as guidance determine Ipp, Ipk, Iavg, Irms, and Amplitude of a non-symmetrical sine wave with the sinusoidal form equation $i(t)=3+8 \sin (120 \pi t)$

This sinusoidal current has a DC offset of $3 \mathrm{~V}$ and is plotted in Figure 1.16.

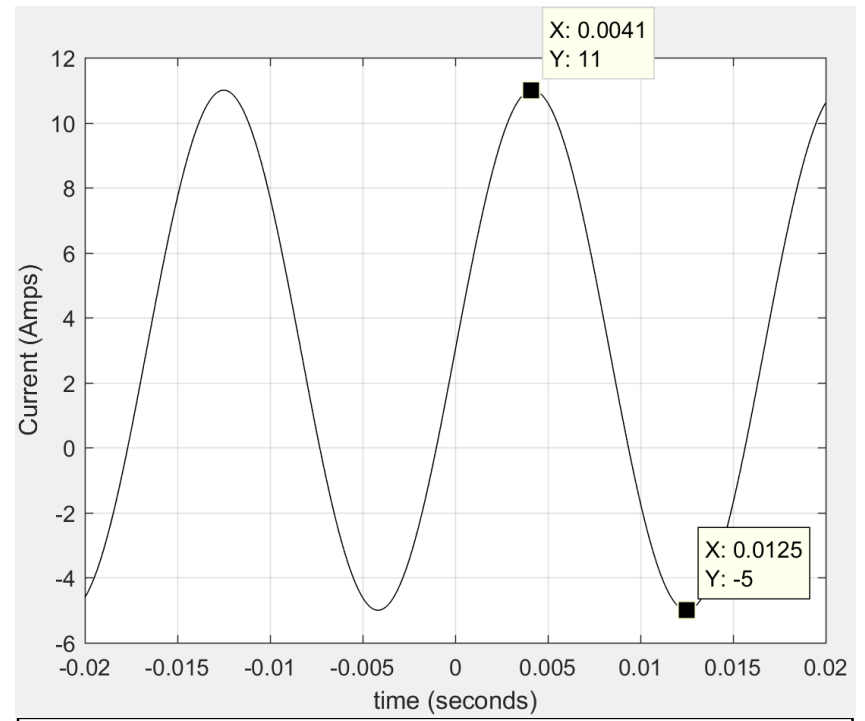

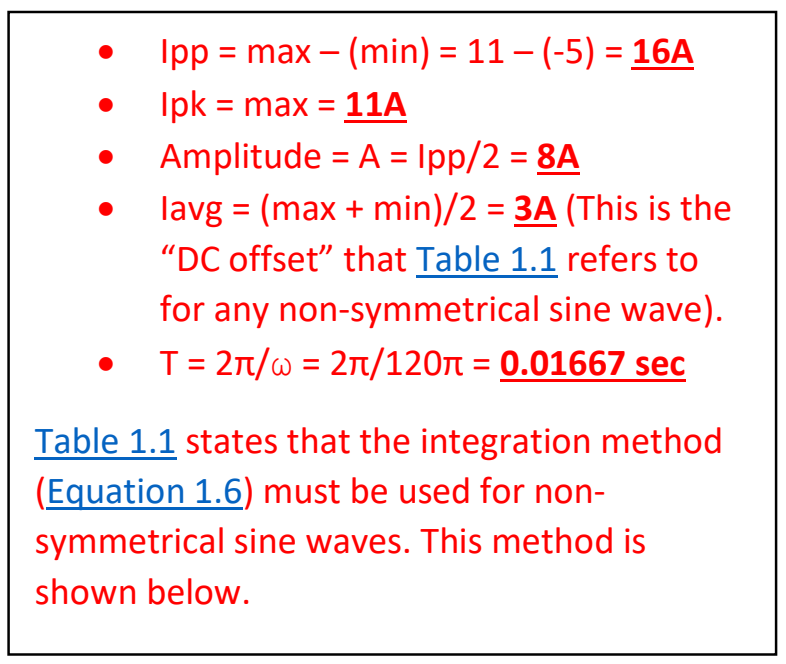

Figure 1.16: Non-symmetrical sine wave in Example 1.4

$i_{r m s}=\sqrt{\frac{1}{T} \int_{0}^{T} i(t)^{2} d t}=\sqrt{\frac{1}{T} \int_{0}^{T}(3+8 \sin (120 \pi t))^{2} d t}$

$i_{r m s}=\sqrt{\frac{1}{0.01667} \int_{0}^{0.01667}\left[9+48 \sin (120 \pi t)+64 \sin (120 \pi t)^{2}\right] d t}=\underline{\mathbf{6 . 4} \mathbf{A}}$ 
The integration in Example 1.4 was solved using the online Wolfram Alpha Integration Widget. Multisim verifies the results of Example 1.4 in Figure 1.17. Since the waveform was not symmetric only the probe box was used for the verification. Also, since the function generator can only output voltage signals the voltage probe was used. Anytime you want to know the Vpp, Vrms, and Vavg (or Ipp, Irms, and lavg) values you can use this method even if it is a current waveform. If it is a current waveform just replace the Volts units with Amps.
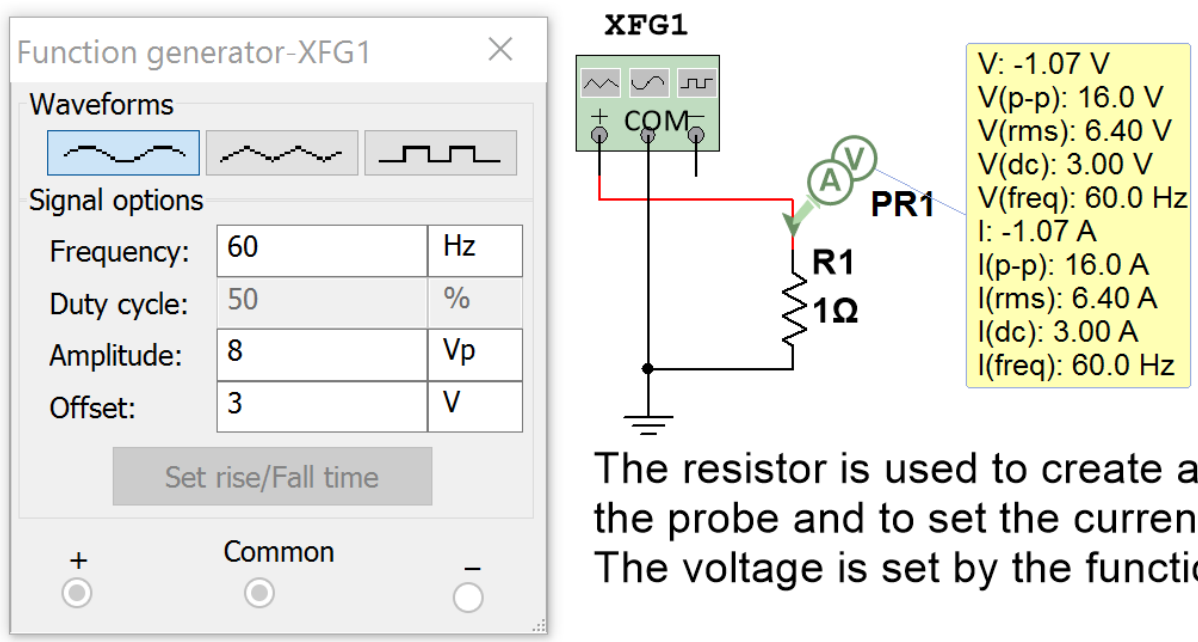

The resistor is used to create a node for the probe and to set the current level. The voltage is set by the function generator.

Figure 1.17: Multisim Verification of Values Calculated in Example 1.4. Note: $I r m s=6.4 \mathrm{Vrms} / 1 \Omega=6.4 \mathrm{~A}$

\section{Section 1.4.2 - Example Calculations of Vpp, Vpk, Vavg, and Vrms for Square Waves}

The term "square wave" in this eBook will be used to define any signal that has a periodic series of rectangular pulses. The most common square wave is one that has a period with one rectangular pulse above the $x$-axis (high pulse) and one below the $x$-axis (low pulse) as shown in Example 1.5. An important parameter for square waves with only one high and one low pulse per period is the duty cycle (DC), which is defined in Equation 1.10.

$$
[1.10] D C=\frac{t_{\text {high }}}{t_{\text {high }}+t_{\text {low }}}
$$

Example 1.5) Using Table 1.1 as guidance, determine Vpp, Vpk, Vavg, and Vrms for the following symmetric $50 \%$ duty cycle square wave.

The cursors on the scope show the high time $50 \mathrm{~ms}$ and since the duty cycle is $50 \%$ the low time is also $50 \mathrm{~ms}$. The period is equal to $\mathrm{t}_{\text {high }}+\mathrm{t}_{\text {low }}$

- $\mathrm{T}=50 \mathrm{~ms}+50 \mathrm{~ms}=100 \mathrm{~ms}=0.1 \mathrm{sec}$.

- $\quad \mathrm{Vpk}=\underline{\mathbf{3 V}}$ (Max voltage on the plot)

- $\mathrm{Vpp}=\max -\min =3-(-3)=\underline{6 \mathrm{~V}}$

- $\quad$ Vavg $=0$ (symmetrical square wave in Table 1.1)

Solve for Vrms using the modified area method

1) Square peaks of each area: $\alpha 1=(3)^{2}=9 V^{2} \quad \alpha 2=(-3)^{2}=9 V^{2}$

2) $\alpha 1 \cdot \mathrm{t} 1=9 \mathrm{~V}^{2} \cdot 0.05 \mathrm{~s}=0.45 \mathrm{~V}^{2} \mathrm{~s} \quad \alpha 2 \cdot \mathrm{t2}=9 \mathrm{~V}^{2} \cdot 0.05 \mathrm{~s}=0.45 \mathrm{~V}^{2} \mathrm{~s}$

3) Total modified area $=\alpha 1 \cdot \mathrm{t} 1+\alpha 2 \cdot \mathrm{t2}=0.45 \mathrm{~V}^{2} \mathrm{~s}+0.45 \mathrm{~V}^{2} \mathrm{~s}=0.9 \mathrm{~V}^{2} \mathrm{~s}$

4) $0.9 \mathrm{~V}^{2} \mathrm{~s} / \mathrm{T}=0.9 \mathrm{~V}^{2} \mathrm{~s} / 0.1 \mathrm{~s}=9 \mathrm{~V}^{2}$

5) $\mathrm{Vrms}=\operatorname{sqrt}\left(9 \mathrm{~V}^{2}\right)=\underline{3 \mathrm{~V}}$

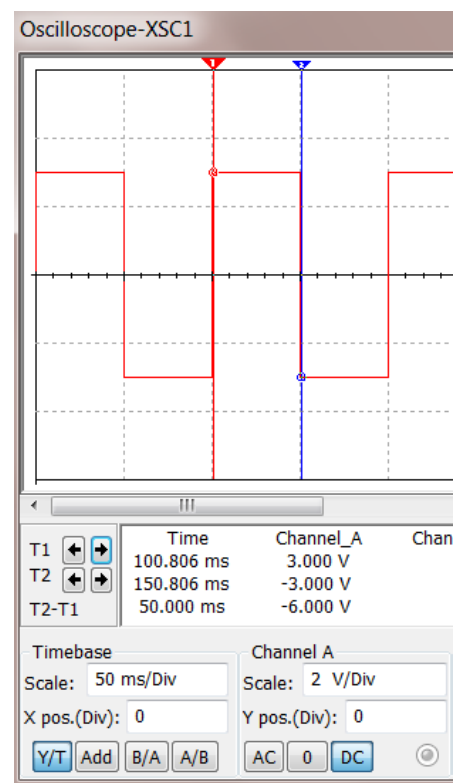

The calculations in Example 1.5 are verified in Figure 1.18. The Duty Cycle in the XFG only affects square waves. 


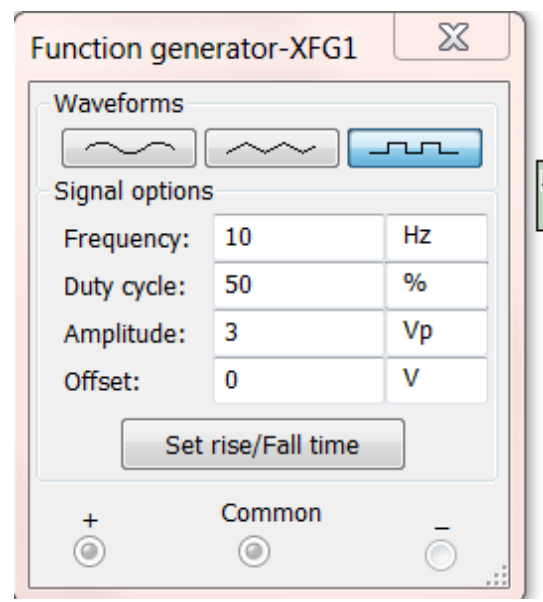

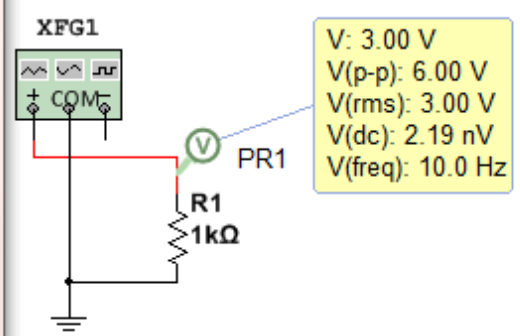

The resistor is only used to create a node for the probe. It has no effect on the results.

\section{Figure 1.18: Multisim Verification of Values Calculated in Example 1.5}

Example 1.6) Using Table 1.1 as guidance, determine Vpp, Vpk, Vavg, and Vrms for the following non-symmetric $50 \%$ duty cycle square wave that has a DC offset of $2 \mathrm{~V}$.

- $\mathrm{T}=50 \mathrm{~ms}+50 \mathrm{~ms}=100 \mathrm{~ms}=0.1 \mathrm{sec}$.

- $\quad \mathrm{Vpk}=\underline{\mathbf{5 V}}$ (Max voltage on the plot)

- $\mathrm{Vpp}=\max -\min =5-(-1)=\underline{6 \mathrm{~V}}$ (Verified in Figure 1.19)

Solve for Vavg using the area method

1) $A_{l}=5 \mathrm{~V} \cdot 0.05 \mathrm{~s}=0.25 \mathrm{Vs} \quad A_{\|}=-1 \mathrm{~V} \cdot 0.05 \mathrm{~s}=-0.05 \mathrm{Vs}$

2) $A_{\text {Total }}=A_{l}+A_{\|}=0.25 \mathrm{Vs}+-0.05 \mathrm{Vs}=0.2 \mathrm{Vs}$

3) $\operatorname{Vavg}=A_{\text {Total }} / \mathrm{T}=0.2 \mathrm{Vs} / 0.1 \mathrm{~s}=\underline{\mathbf{2 V}}$ (Verified in Figure 1.19)

Solve for Vrms using the modified area method

1) Square peaks: $\quad \alpha 1=(5)^{2}=25 \mathrm{~V}^{2} \quad \alpha 2=(-1)^{2}=1 \mathrm{~V}^{2}$

2) $\alpha 1 \cdot \mathrm{t} 1=25 \mathrm{~V}^{2} \cdot 0.05 \mathrm{~s}=1.25 \mathrm{~V}^{2} \mathrm{~s} \quad \alpha 2 \cdot \mathrm{t2}=1 \mathrm{~V}^{2} \cdot 0.05 \mathrm{~s}=0.05 \mathrm{~V}^{2} \mathrm{~s}$

3) Total Modified Area $=\alpha 1 \cdot \mathrm{t} 1+\alpha 2 \cdot \mathrm{t} 2=1.25 \mathrm{~V}^{2} \mathrm{~s}+0.05 \mathrm{~V}^{2} \mathrm{~s}=1.3 \mathrm{~V}^{2} \mathrm{~s}$

4) $1.3 \mathrm{~V}^{2} \mathrm{~s} / \mathrm{T}=1.3 \mathrm{~V}^{2} \mathrm{~s} / 0.1 \mathrm{~s}=13 \mathrm{~V}^{2}$

5) $\mathrm{Vrms}=\operatorname{sqrt}\left(13 \mathrm{~V}^{2}\right)=\underline{\mathbf{3 . 6 1 \mathrm { V }}}($ Verified in Figure 1.19)$)$
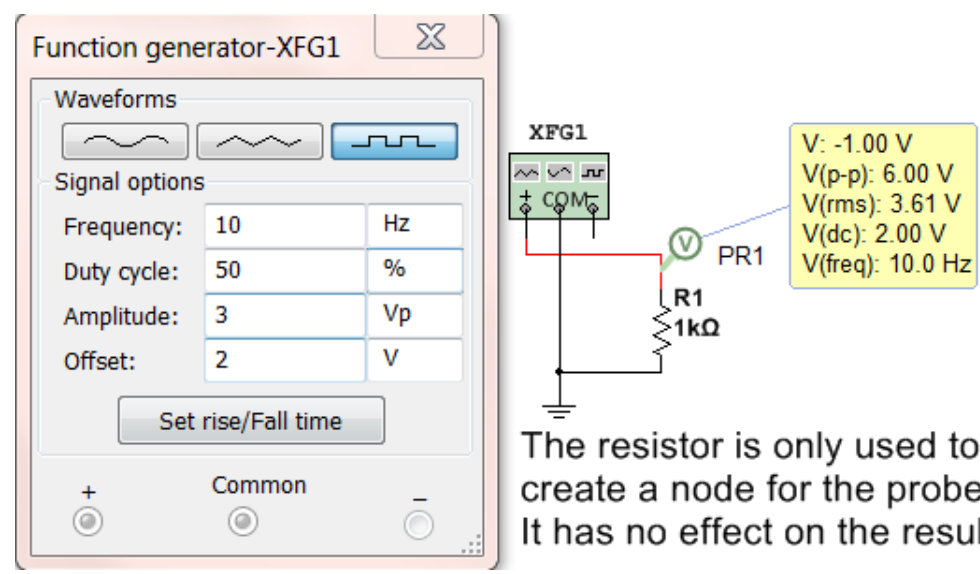

The resistor is only used to create a node for the probe. It has no effect on the results.

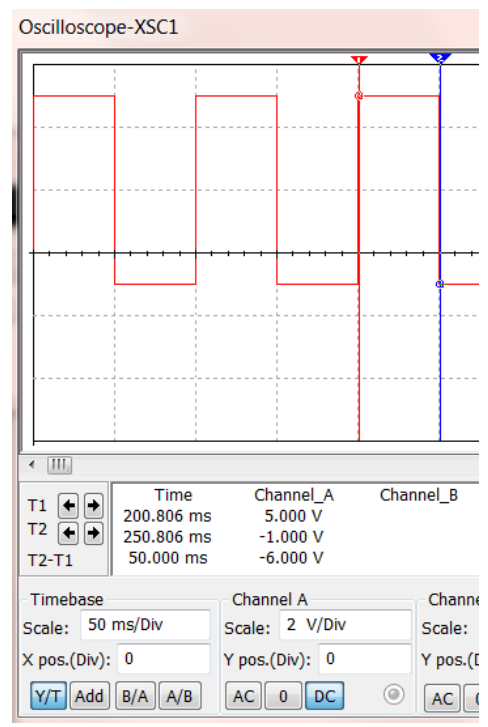

Figure 1.19: Multisim Verification of Values Calculated in Example 1.6 
Example 1.7) Using Table 1.1 as guidance, determine Vpp, Vpk, Vavg, and Vrms for the following non-symmetric $80 \%$ duty cycle square wave that has a DC offset of $-2 \mathrm{~V}$.

- $t_{\text {high }}$ is shown to be $\sim 80 \mathrm{~ms}$ on the scope plot.

- Solving for $\mathrm{t}_{\text {low }} \mathrm{using}$ Equation 1.10 gives $\mathrm{t}_{\text {low }}=20 \mathrm{~ms}$

- $\mathrm{T}=80 \mathrm{~ms}+20 \mathrm{~ms}=100 \mathrm{~ms}=0.1 \mathrm{sec}$.

- $\quad \mathrm{Vpk}=\underline{\mathbf{3 V}}$ (Max voltage on the plot)

- $\mathrm{Vpp}=\max -\min =3-(-7)=\underline{10 \mathrm{~V}}$ (Verified in Figure 1.20)

Solve for Vavg using the area method

1) $A_{l}=3 \mathrm{~V} \cdot 0.08 \mathrm{~s}=0.24 \mathrm{Vs} \quad A_{\|}=-7 \mathrm{~V} \cdot 0.02 \mathrm{~s}=-0.14 \mathrm{Vs}$

2) $A_{\text {Total }}=A_{l}+A_{l l}=0.24 \mathrm{Vs}+-0.14 \mathrm{Vs}=0.1 \mathrm{Vs}$

3) $\operatorname{Vavg}=A_{\text {Total }} / T=0.1 \mathrm{Vs} / 0.1 \mathrm{~s}=\underline{\mathbf{1 V}}$ (Verified in Figure 1.20)

Solve for Vrms using the modified area method

1) Square peaks: $\quad \alpha 1=(3)^{2}=9 V^{2} \quad \alpha 2=(-7)^{2}=49 V^{2}$

2) $\alpha 1 \cdot \mathrm{t} 1=9 \mathrm{~V}^{2} \cdot 0.08 \mathrm{~s}=0.72 \mathrm{~V}^{2} \mathrm{~s} \quad \alpha 2 \cdot \mathrm{t} 2=49 \mathrm{~V}^{2} \cdot 0.02 \mathrm{~s}=0.98 \mathrm{~V}^{2} \mathrm{~s}$

3) Total Modified Area $=\alpha 1 \cdot \mathrm{t} 1+\alpha 2 \cdot \mathrm{t} 2=0.72 \mathrm{~V}^{2} \mathrm{~s}+0.98 \mathrm{~V}^{2} \mathrm{~s}=1.7 \mathrm{~V}^{2} \mathrm{~s}$

4) $1.7 \mathrm{~V}^{2} \mathrm{~s} / \mathrm{T}=1.7 \mathrm{~V}^{2} \mathrm{~s} / 0.1 \mathrm{~s}=17 \mathrm{~V}^{2}$

5) $\operatorname{Vrms}=\operatorname{sqrt}\left(17 \mathrm{~V}^{2}\right)=\underline{4.12 \mathrm{~V}}($ Verified in Figure 1.20)

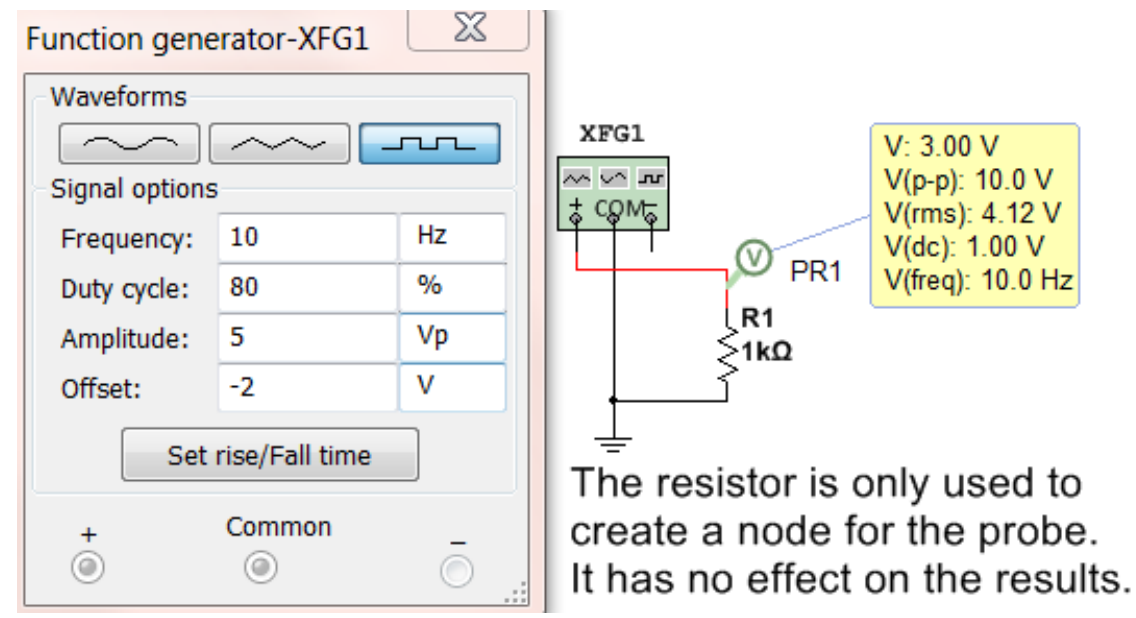

Figure 1.20: Multisim Verification of Values Calculated in Example 1.6

Examples 1.5, 1.6, and 1.7 show realistic square waves that have one high pulse and one low pulse per period, but you can also use these methods to determine the Vpp, Vpk, Vavg, and Vrms for a square wave that has multiple high and low pulses (see Example 1.8). Unfortunately, Multisim cannot be used to verify answers when there is more than one high and one low pulse. 
Example 1.8) One cycle of a periodic square wave is shown in the figure below. Using Table 1.1 as guidance, determine the Vpp, Vpk, Vavg, and Vrms values for this square wave.
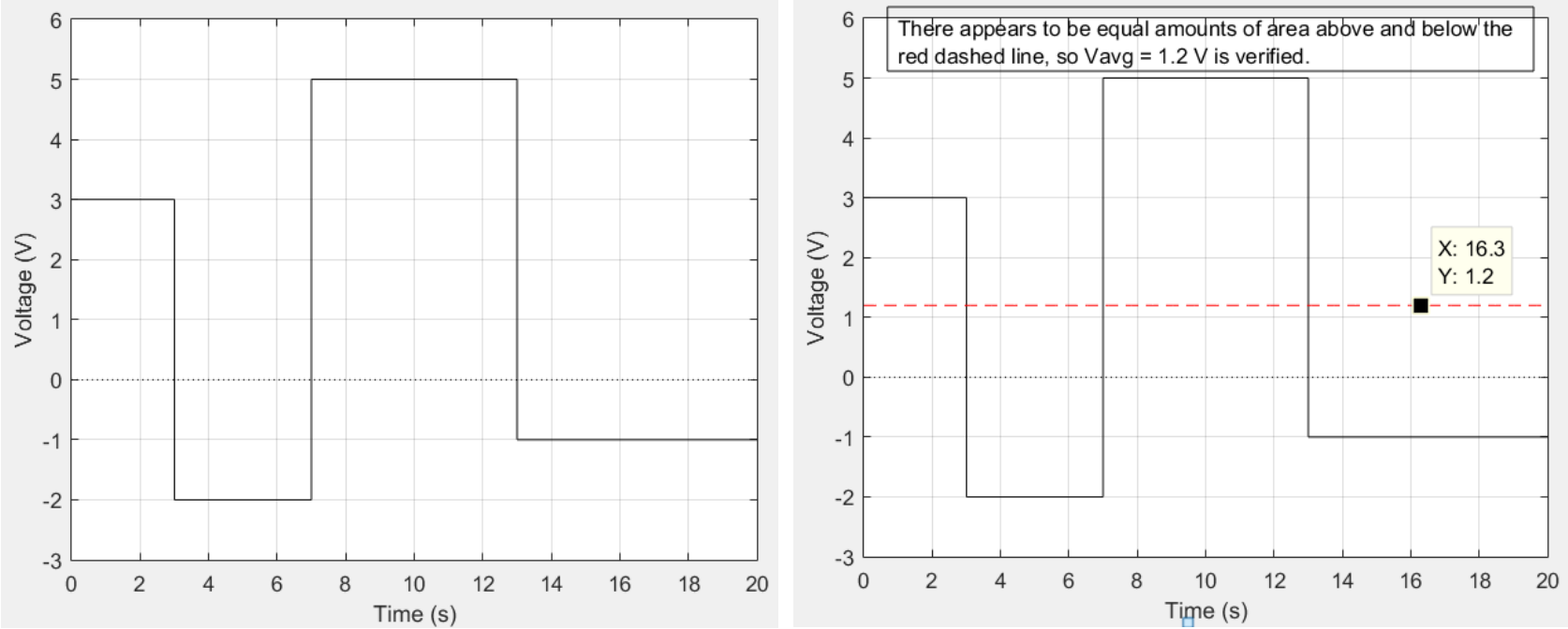

Figure 1.21: One cycle of a periodic square wave in Example 1.8 (Left), Average Value shown in right plot.

- $\mathrm{T}=20$ sec. (From plot)

- $\quad \mathrm{Vpk}=\underline{\mathbf{5 V}}$ (Max voltage on the plot)

- $V p p=\max -\min =5-(-2)=\underline{7 V}$

Solve for Vavg using the area method (labeling the areas from left to right in ascending order)

1) $A_{l}=3 \mathrm{~V} \cdot 3 \mathrm{~s}=9 \mathrm{Vs} \quad \mathrm{A}_{\|}=-2 \mathrm{~V} \cdot 4 \mathrm{~s}=-8 \mathrm{Vs} \quad A_{\| I}=5 \mathrm{~V} \cdot 6 \mathrm{~s}=30 \mathrm{Vs} \quad A_{I V}=-1 \mathrm{~V} \cdot 7 \mathrm{~s}=-7 \mathrm{Vs}$

2) $A_{\text {Total }}=A_{l}+A_{l l}+A_{l l}+A_{I V}=9 V s-8 V s+30 V s-7 V s=24 V s$

3) $\operatorname{Vavg}=\mathrm{A}_{\text {Total }} / \mathrm{T}=24 \mathrm{Vs} / 20 \mathrm{~s}=\underline{1.2 \mathrm{~V}}$ (See the plot above-right with the red dashed line)

Solve for Vrms using the modified area method

1) Square peaks: $\alpha 1=(3)^{2}=9 \mathrm{~V}^{2} \quad \alpha 2=(-2)^{2}=4 \mathrm{~V}^{2} \quad \alpha 3=(5)^{2}=25 \mathrm{~V}^{2} \quad \alpha 4=(-1)^{2}=1 \mathrm{~V}^{2}$

2) $\alpha 1 \cdot t 1=9 V^{2} \cdot 3 s=27 V^{2} s \quad \alpha 2 \cdot t 2=4 V^{2} \cdot 4 s=16 V^{2} s \quad \alpha 3 \cdot t 3=25 V^{2} \cdot 6 s=150 V^{2} s \quad \alpha 4 \cdot t 4=1 V^{2} \cdot 7 s=7 V^{2} s$

3) Total Modified Area $=\alpha 1 \cdot t 1+\alpha 2 \cdot t 2+\alpha 3 \cdot t 3+\alpha 4 \cdot t 4=27 \mathrm{~V}^{2} s+16 \mathrm{~V}^{2} s+150 \mathrm{~V}^{2} s+7 \mathrm{~V}^{2} s=200 \mathrm{~V}^{2} \mathrm{~s}$

4) $200 \mathrm{~V}^{2} \mathrm{~s} / \mathrm{T}=200 \mathrm{~V}^{2} \mathrm{~s} / 20 \mathrm{~s}=10 \mathrm{~V}^{2}$

5) $\operatorname{Vrms}=\operatorname{sqrt}\left(10 \mathrm{~V}^{2}\right)=\underline{\mathbf{3 . 1 6 \mathrm { V }}}$

Section 1.4.3 - Example Calculations of Vpp, Vpk, Vavg, and Vrms for Triangle Waves

Triangle waves aren't nearly as common as sine waves and square waves, but they are present from time to time in AC circuits. One example is when high frequency square wave pulses are transmitted in a circuit that have such narrow pulses that the components can't handle the speed due to slew rate $(\Delta v / \Delta t)$ limitations and the square wave is converted to a waveform that looks more like a triangle. A saw tooth is another popular triangular waveform that ramps upwards until it reaches its positive peak (just like a standard triangle wave) and then drops instantaneously to its negative peak (like a standard square wave). The term "triangle wave" in this eBook will be used to define any signal that has a periodic series of triangular pulses. The most common triangle wave is one where each period has one triangular section above the $x$-axis and one below the $x$-axis with a slope that has a constant magnitude, but alternates signs as shown in Example 1.9. 
Example 1.9) Using Table 1.1 as guidance, determine Vpp, Vpk, Vavg, and Vrms for the following symmetric triangle wave. Note: The Duty Cycle in the function generator settings below also has an effect on triangle waves. If the Duty cycle is set to a minimum value of 1 then the triangle wave will look like a "saw tooth" wave that will ramp up to its peak nearly instantaneously and drop down to its negative peak more slowly. The reverse will happen if a Duty Cycle is set to the maximum value of 99. Stop and restart the simulation when making changes.

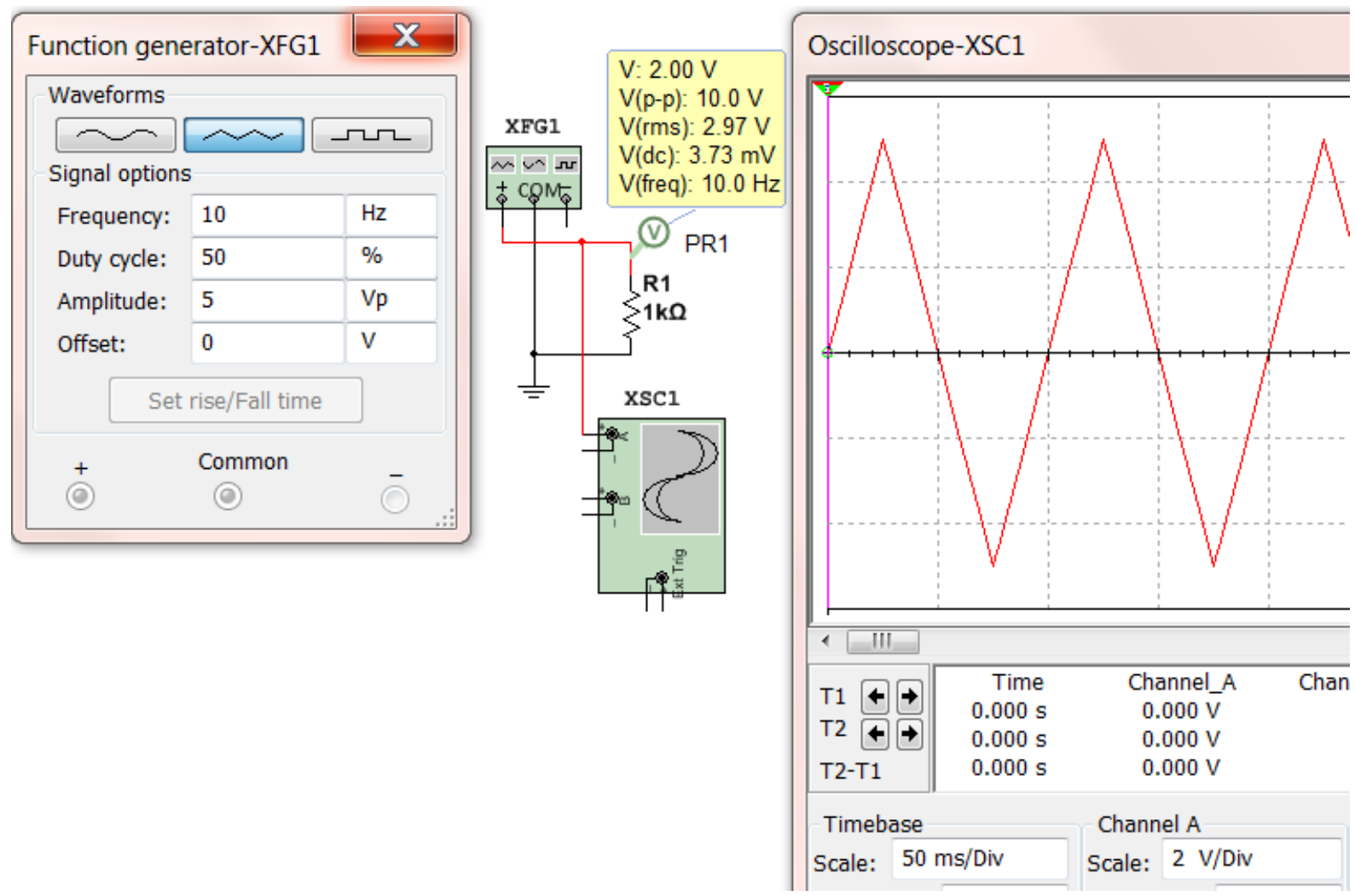

Figure 1.22: Multisim function generator settings and scope print for triangle wave in Example 1.9.

- $\mathrm{T}=1 / \mathrm{F}=1 / 10=0.1 \mathrm{sec}$.

- $\mathrm{Vpk}=\underline{\mathbf{5 V}}$ (Max voltage on the oscilloscope plot)

- $\mathrm{Vpp}=\max -\min =5-(-5)=\underline{\mathbf{1 0 V}}$ (Verified with Multisim voltage probe)

- $\quad \operatorname{Vavg}=0$ (symmetrical square wave in Table 1.1) (Verified as $0 \mathrm{~V}$ with Multisim voltage probe)

For triangle waves the integration method (Equation 1.6) must be used for Vrms. There is no simplification.

- Each line needs to be broken up in separate functions over one period. The easiest way to do this is determine the slope $(5 / 0.025=200)$ and break the waveform up into 2 sections.

- From $\mathrm{t}=0$ to $\mathrm{T} / 2, \mathrm{v} 1(\mathrm{t})=200 \cdot \mathrm{t} \quad$ and From $\mathrm{t}=\mathrm{T} / 2$ to $\mathrm{T}, \mathrm{v} 2(\mathrm{t})=-200 \cdot \mathrm{t}$

$$
\begin{aligned}
& v_{r m s}=\sqrt{\frac{1}{T} \int_{0}^{T} v(t)^{2} d t}=\sqrt{\frac{1}{T}\left[\int_{-T / 4}^{T / 4}(200 \cdot t)^{2} d t+\int_{T / 4}^{3 T / 4}(10-200 \cdot t)^{2} d t\right]} \\
& v_{r m s}=\sqrt{\frac{1}{0.1}\left\{\left[13333.333 \cdot t^{3}\right]_{-0.025}^{0.025}+\left[100 \cdot t-2000 \cdot t^{2}+13333.33 \cdot t^{3}\right]_{0.025}^{0.075}\right.}
\end{aligned}
$$


$v_{r m s}=\sqrt{8.3333}=2.89 \mathrm{~V} *$ The Multisim probe value was very close to this value $(2.97 \mathrm{~V})$.

Example 1.10) Using Table 1.1 as guidance, determine Vpp, Vpk, and Vavg for the following figure that shows one cycle of a non-symmetric periodic triangle wave. Note: The Multisim function generator can't be used to create a complicated waveform like this. Since calculating Vrms for triangle waves is arduous and not frequently necessary this example will leave it out and only solve Vpk, Vpp, and Vavg.

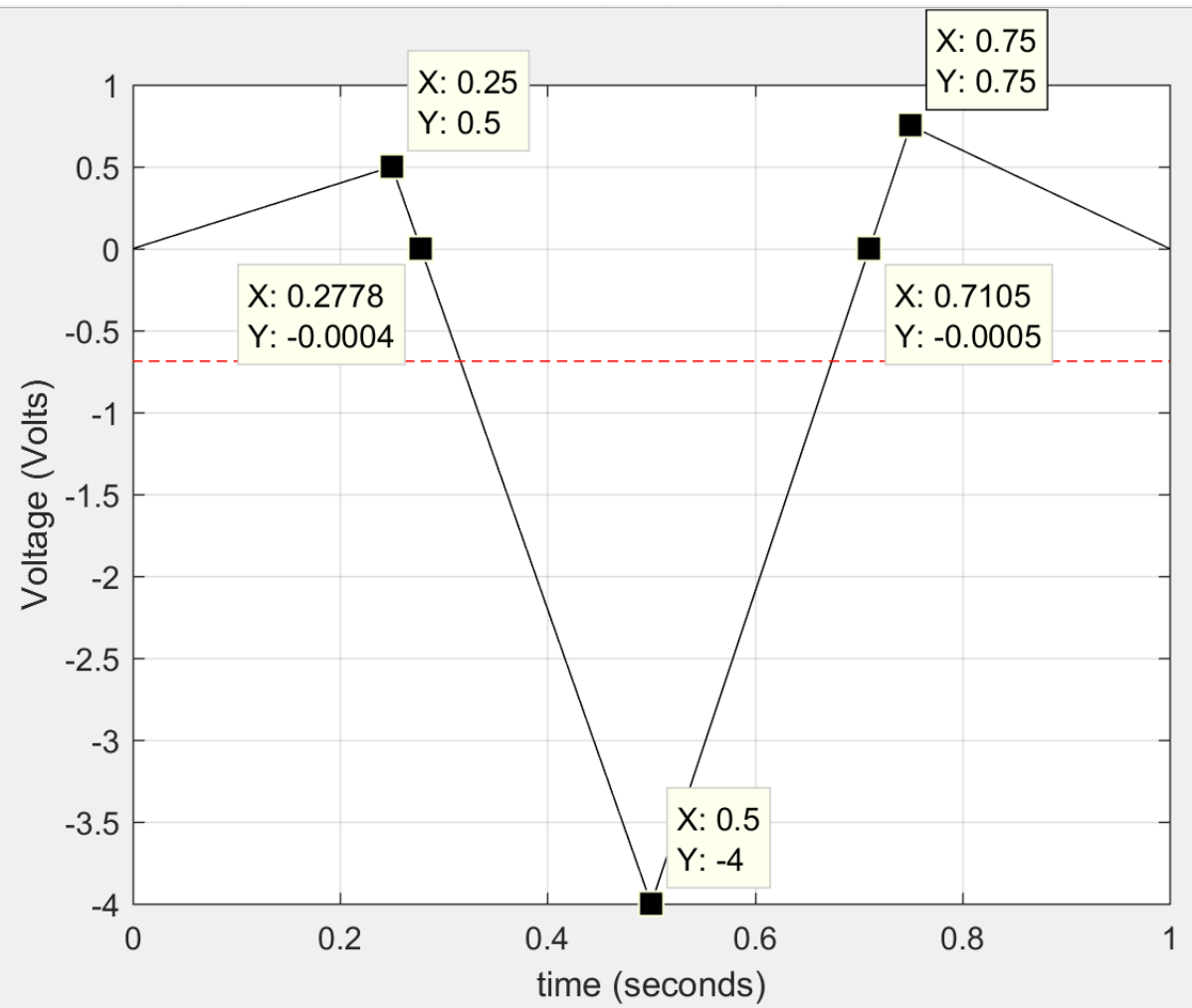

Figure 1.23: Triangle wave for Example 1.10.

- $T=1 \mathrm{sec}$.

- $\mathrm{Vpk}=\underline{\mathbf{0 . 7 5 \mathrm { V }}}$ (Max voltage)

- $\quad \mathrm{pp}=\max -\min =0.75-(-4)=\underline{4.75 \mathrm{~V}}$

Solve for Vavg using the area method (labeling the areas from left to right of each right angle triangle in ascending order). Assume the $Y$ values shown in Figure 1.23 that are -.0004 and -.0005 are equal to zero.

1) $A_{l}=1 / 2 b \cdot h=1 / 2(0.25-0) \mathrm{sec} \cdot 0.5 \mathrm{~V}=0.0625 \mathrm{Vs}$

$A_{\| l}=1 / 2 b \cdot h=1 / 2(0.2778-0.25) \mathrm{sec} \cdot 0.5 \mathrm{~V}=1 / 20.0278 \mathrm{sec} \cdot 0.5 \mathrm{~V}=0.00695 \mathrm{Vs}$

$A_{\| I I}=1 / 2 b \cdot h=1 / 2(0.5-0.2778) \mathrm{sec} \cdot(-4 \mathrm{~V})=1 / 20.222 \mathrm{sec} \cdot(-4 \mathrm{~V})=-0.4444 \mathrm{Vs}$

$A_{I V}=1 / 2 b \cdot h=1 / 2(0.7105-0.5) \mathrm{sec} \cdot(-4 \mathrm{~V})=1 / 20.2105 \mathrm{sec} \cdot(-4 \mathrm{~V})=-0.421 \mathrm{Vs}$

$A_{V}=1 / 2 b \cdot h=1 / 2(0.75-0.7105) \mathrm{sec} \cdot 0.75 \mathrm{~V}=1 / 20.0395 \mathrm{sec} \cdot 0.75 \mathrm{~V}=0.014813 \mathrm{Vs}$

$A_{V I}=1 / 2 b \cdot h=1 / 2(1-0.75) \mathrm{sec} \cdot 0.75 \mathrm{~V}=1 / 20.25 \mathrm{sec} \cdot 0.75 \mathrm{~V}=0.09375 \mathrm{Vs}$

2) $A_{\text {Total }}=A_{l}+A_{I I}+A_{I I I}+A_{I V}+A_{V}+A_{V I}=0.0625+0.00695-0.4444-0.421+0.014813+0.09375=\underline{-0.6874 V s}$

3) $\operatorname{Vavg}=A_{\text {Total }} / T=-0.6874 \mathrm{Vs} / 1 \mathrm{~s}=\underline{\mathbf{- 0 . 6 8 7 4} \mathrm{V}}$ (See the plot above with the red dashed line shows this value looks like the midpoint between the positive and negative parts of the waveform. 


\section{Module 2 - AC Circuits Math Background}

Module 1 described common types of AC sources. From this point on the focus will be solely on sinusoidal inputs that were represented in Equation 1.1 in Section 1.2 as the following:

- Sinusoidal Form $\rightarrow v(t)=A \cdot \sin (\omega \cdot t+\theta)$

Module 2 will provide the math background needed to be able to solve RLC circuits with AC sources. The first mathematical simplification is to represent the sinusoidal signal as a "phasor" that has a set amplitude and phase. The "phasor form" of a sinusoid is the amplitude and phase represented in polar form.

\section{- Phasor Form $\rightarrow \mathrm{V}=\mathrm{A}<\boldsymbol{\theta}$}

Capital letters are used for Phasor variables and lower case letters are used for sinusoidal form variables. Phasor form is convenient because all signals in any RLC circuit will have the same frequency as the source (or input) frequency. For example, the following figure shows an RC circuit and the input and output voltage signals. Notice that the frequencies of $\mathbf{v}_{\text {in }}$ and $\mathbf{v}_{\text {out }}$ are the same, but both the amplitude and phase change.

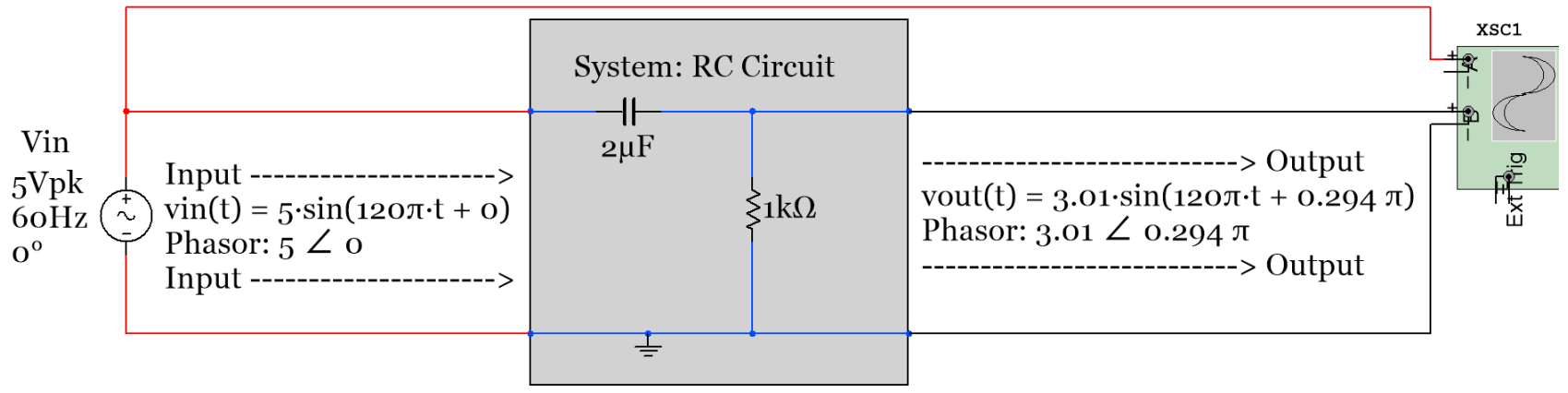

Figure 2.1: Example showing how an RC circuit affects the amplitude and phase of a sinusoidal input.

Oscilloscope-XSC1

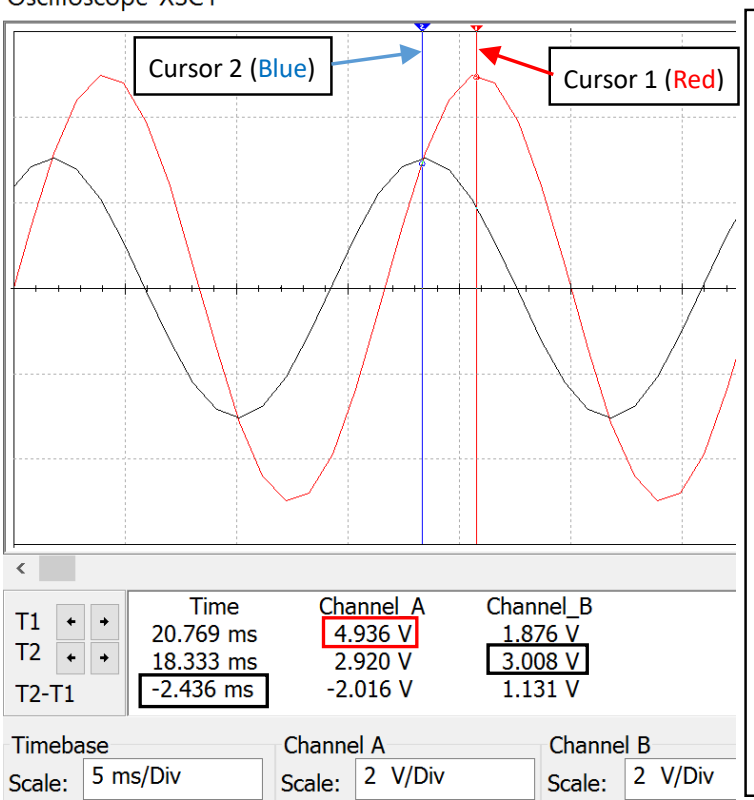

* The Red waveform is the input waveform with a peak voltage of $\sim 5 \mathrm{~V}$ and a phase of 0 . Phasor $=5 \angle 0$

* The Black waveform is the output that has an amplitude of $\sim 3 V$ and a $0.294 \pi$ phase $\rightarrow 3 \angle 0.294 \pi$

* The phase can be verified using the time cursors of the oscilloscope plot. The time difference between the two peaks is equal to $\sim 2.436 \mathrm{~ms}$. The value is negative because the peak of the output is to the left of the input. The time shift is measured to be: $t_{s}=-2.436 \mathrm{~ms}$

* Eq. 1.3) $\theta=-\omega \cdot t_{s}=-(120 \pi \cdot-2.436 \mathrm{~ms})=+0.292 \pi$

This is very close to the actual phase shift of $+0.294 \pi$. This slight difference comes from the inability to precisely measure the time shift on the scope.

Figure 2.2: Oscilloscope plot of Figure 2.1 
Figure 2.2 shows how the phase shift and amplitude can be determined using Multisim, but there is some measurement error due to the limited resolution of the scope plots. The frequency could also be found in the scope plot by measuring the period and using the equation $f=1 / T$. It can be seen that the frequency stays the same in Figure 2.2 since the output and input always have the same time shift between them. Therefore, the phasor can be used to describe the signal, and the math required to determine the output phasor can be accomplished using simple vector operations of the phasors instead of trigonometry. The frequency can be removed from the calculations. Example 3.1 in Module 3 shows how to calculate the output phasor $(3 \angle 0.294 \pi)$ for the circuit in Figure 2.1, but before getting to that it will be beneficial for many students at this point to review vector addition, subtraction, multiplication, and division for vectors in polar and rectangular form. If your skills in this area are not solid then you will have a very difficult time solving the circuits in Module 3.

The vectors in this section will be used to represent currents, voltages, admittances, and impedances in Module 3. The impedance for capacitors and inductors are $-90^{\circ}$ and $+90^{\circ}$ out of phase respectively with resistors which leads to the xy plot in Figure 2.3 where the $y$-axis represents the imaginary plane and the $x$-axis represents the real plane. The two types of vector forms are rectangular and polar (Recall: Phasors are in polar form). An example of a vector in quadrant 1 is shown in Figure 2.3 with the variables labeled that are used in rectangular form $(X+j Y)$ and polar form $(A \angle \theta)$.
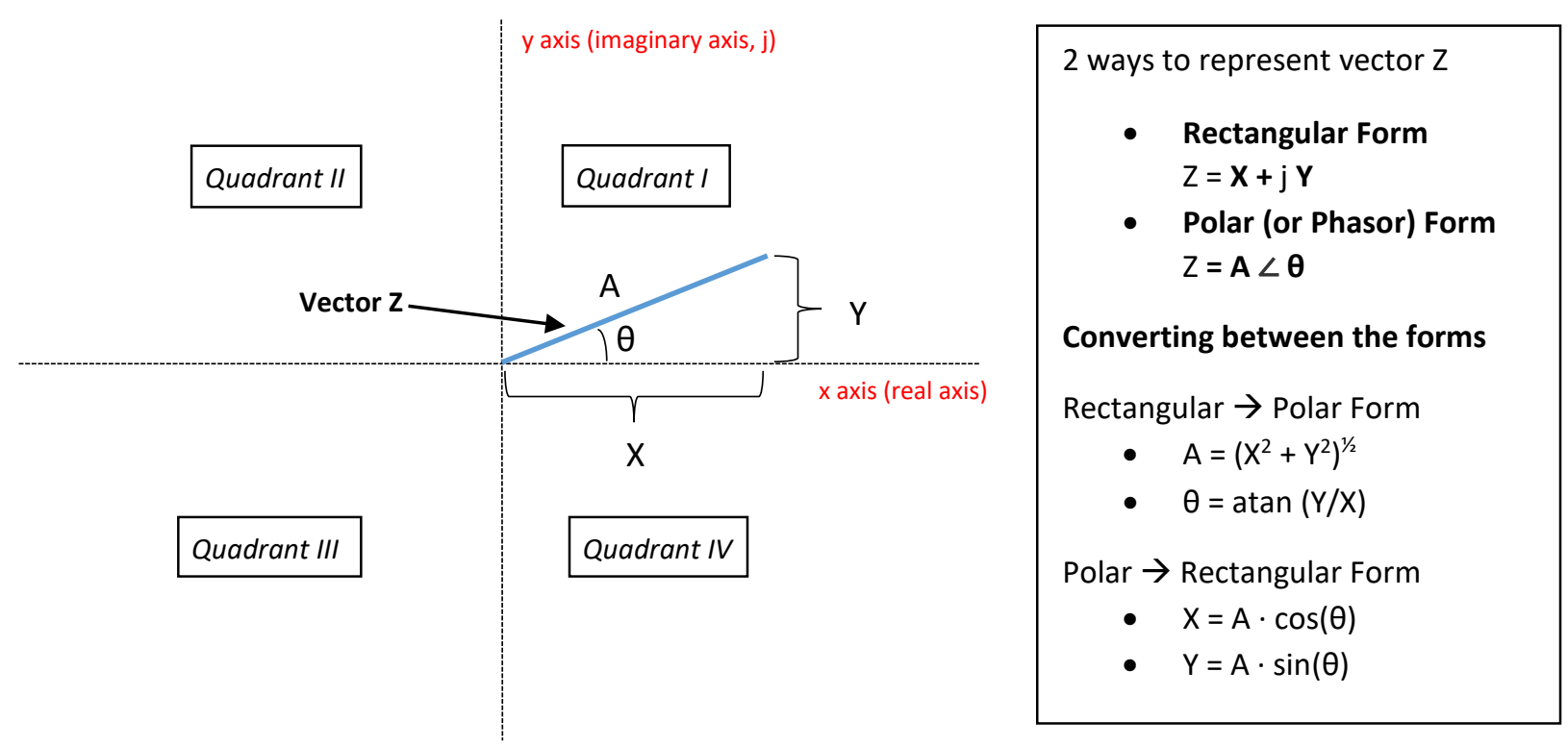

Figure 2.3: $\mathrm{XY}$ plot of vector $\mathrm{Z}$ (Note: $\mathrm{Z}$ is just the variable used for the vector. Any variable can be used). In polar (or Phasor) form the angle, $\theta$, is always taken with respect to the positive real $x$-axis such that a unit length vectors lying on the positive or negative $\mathrm{x}$ or $\mathrm{y}$ axes can be represented as follows:

- $Z_{1}=1 \angle 0^{\circ}$ lies on the positive (+) side of the $x$-axis and has a rectangular form as $Z_{1}=\mathbf{1 + j} \cdot \mathbf{0}$ or just $Z_{1}=\mathbf{1}$

- $Z_{2}=\mathbf{1} \angle \mathbf{9 0 ^ { \circ }}=\mathbf{1} \angle \boldsymbol{\pi} / \mathbf{2}$ lies on the $(+)$ side of the $y$-axis and has a rectangular form as $Z_{\mathbf{2}}=\mathbf{0}+\mathbf{j} \cdot \mathbf{1}$ or $Z_{\mathbf{2}}=\mathbf{j}$

- $Z_{3}=1 \angle 180^{\circ}=1 \angle \pi$ lies on the $(-)$ side of the $x$-axis and has a rectangular form as $Z_{3}=-\mathbf{1}+\mathbf{j} \cdot \mathbf{0}$ or $Z_{3}=-1$

- $\mathrm{Z}_{4}=\mathbf{1} \angle \mathbf{2 7 0 ^ { \circ }}=\mathbf{1} \angle-\pi / 2$ lies on the $(-)$ side of the $\mathrm{y}$-axis and has a rectangular form as $\mathrm{Z}_{4}=\mathbf{0}-\mathbf{j} \cdot \mathbf{1}$ or $\mathrm{Z}_{4}=-\mathbf{j}$

Note: The angles for $Z_{1}$ to $Z_{4}$ were given in degrees and signified by a degree symbol, but when converting it to radians no symbol is needed. It is always assumed the angle is in radians if there is no degree symbol. When radians are used to list the angle it is preferred to put the radians value as a fraction of $\pi$. Always represent a phasor such that the angle is between $-180^{\circ}$ and $+180^{\circ}$ (or $-\pi$ and $+\pi$ ). If the angle, $\theta$, is greater than $\pi$ then 
subtract $2 \pi$ until $\theta$ is between $-\pi$ and $+\pi$. Conversely, if $\theta$ is less than $-\pi$ then add $2 \pi$ until $\theta$ is between $-\pi$ and $+\pi$.

Converting between rectangular and polar form is something that needs to be done frequently when solving AC circuits because:

- When adding or subtracting vectors rectangular form is much easier.

- When multiplying or dividing vectors polar form is much easier.

When combining impedances or admittances or when using a KVL or KCL, vectors are added or subtracted so all vectors should be converted to rectangular form. When using Ohm's law vectors are multiplied or divided so all vectors are converted to polar form.

Converting from polar to rectangular form (as shown in Example 2.1) is straightforward, but converting from rectangular to polar form (as shown in Example 2.2) is trickier due to the ambiguity of the angle. For example, when $Y$ and $X$ have the same value the arc tangent (atan) of 1 is equal to $45^{\circ}$, but this situation can occur in two different situations: Quadrant I where $X$ and $Y$ are positive or Quadrant III where $X$ and $Y$ are negative. If the vector was in quadrant I then $\theta=+45^{\circ}$, but if the vector was in quadrant III then $\theta=-180^{\circ}+45^{\circ}=-135^{\circ}$. In order to avoid mistakes when calculating $\theta$ the "reference angle" $\left(\boldsymbol{\theta}_{\text {REF }}\right)$ should be found and then the quadrant should be determined based on the signs of $X$ and $Y$ as shown below.

- $\boldsymbol{\theta}_{\mathrm{REF}}=\operatorname{atan}(|\mathrm{Y}| /|\mathrm{X}|) \quad$ Note: Take the absolute value of $\mathrm{Y}$ and $\mathrm{X}$ so $\theta_{\mathrm{REF}}$ is always between $0^{\circ}$ and $90^{\circ}$.

Next, the reference angle can be used to find the actual angle by knowing what quadrant the vector is in.

I. Quadrant I $(X>0, Y>0): \quad \theta=\theta_{\text {REF }}$

II. Quadrant II $(X<0, Y>0): \quad \theta=180^{\circ}-\theta_{\text {REF }}$ OR $\pi-\theta_{\text {REF }}$

III. Quadrant III $(X<0, Y<0)$ : $\theta=-180^{\circ}+\theta_{\text {REF }}$ OR $-\pi+\theta_{\text {REF }}$

IV. Quadrant IV $(X>0, Y<0)$ : $\boldsymbol{\theta}=-\boldsymbol{\theta}_{\text {REF }}$

Example 2.1) Convert the following vectors from polar to rectangular form.

$Z_{1}=1.414 \angle 3 \pi / 4 \quad Z_{2}=1.6 \angle \pi / 6 \quad Z_{3}=3 \angle-\pi / 2 \quad Z_{4}=7 \angle 7 \pi$

- $Z_{1}=1.414 \angle 3 \pi / 4 \rightarrow Z_{1}=X+j Y=1.414 \cos (3 \pi / 4)+\mathrm{j} 1.414 \sin (3 \pi / 4)=\underline{\mathbf{- 1}+\mathbf{j} \mathbf{1}}$

- $Z_{2}=1.6 \angle \pi / 6 \rightarrow Z_{2}=X+j Y=1.6 \cos (\pi / 6)+j 1.6 \sin (\pi / 6)=\underline{\mathbf{1 . 3 8 6}+\mathbf{j} 0.8}$

- $Z_{3}=3 \angle-\pi / 2 \rightarrow Z_{3}=X+j Y=3 \cos (-\pi / 2)+j 3 \sin (-\pi / 2)=-j 3$

- $Z_{4}=7 \angle 7 \pi \rightarrow Z_{4}=X+j Y=7 \cos (7 \pi)+j 7 \sin (7 \pi)=\underline{-7}$

Example 2.2) Convert the following vectors from rectangular to polar form.

$Z_{1}=j 2 \quad Z_{2}=-1+j \quad Z_{3}=-3-j 4 \quad Z_{4}=(0,-1)$

- $Z_{1}=\mathrm{j} 2=0+\mathrm{j} 2=X+\mathrm{j} Y$

○ $A=\left[0^{2}+2^{2}\right]^{1 / 2}=\underline{2}$

- $\theta$ lies on the positive $y$-axis so it is equal to $90^{\circ}$ or $\pi / 2$

○ $Z_{1}=0+\mathrm{j} 2 \rightarrow Z_{1}=A \angle \theta=\underline{\mathbf{2}} \angle \pi / 2$

- $Z_{2}=-1+j=X+j Y$

○ $A=\left[(-1)^{2}+1^{2}\right]^{1 / 2}=\underline{1.414}$

○ $\theta_{\text {REF }}=\operatorname{atan}(|1| /|-1|)=\underline{\pi / 4}$

○ $\quad \theta=\pi-\theta_{\text {REF }}($ Quadrant II) $=\underline{3 \pi / 4}$

○ $Z_{2}=-1+j \rightarrow Z_{2}=A \angle \theta=\underline{1.414 \angle 3 \pi / 4}$ 
- $Z_{3}=-3-j 4=X+j Y$

- $A=\left[(-3)^{2}+(-4)^{2}\right]^{1 / 2}=\underline{5}$

- $\theta_{\text {REF }}=\operatorname{atan}(|-4| /|-3|)=53.13^{\circ}(\pi / 180)=.295 \pi$

○ $\theta=-\pi+\theta_{\text {REF }}$ (Quadrant III) $=\underline{-0.705 \pi}$

○ $Z_{3}=\underline{5 \angle-0.705 \pi}$

- $\mathrm{Z}_{4}=(0,-1) \rightarrow$ This $(\mathrm{X}, \mathrm{Y})$ notation simply means $\mathrm{X}=0, \mathrm{Y}=-1$ so $\mathrm{Z}_{4}=0-1 \mathrm{j}$

○ $A=\left[0^{2}+(-1)^{2}\right]^{1 / 2}=\underline{1}$

- $\theta$ is on the negative $y$-axis so it is equal to $-\pi / 2$

○ $Z_{4}=\underline{1 \angle-\pi / 2}$

After mastering the ability to convert vectors between rectangular and polar form it is time to add, subtract, multiply, and divide vectors. When adding or subtracting vectors they should be put in rectangular form and the real parts are added or subtracted and the imaginary parts are added or subtracted separately as shown in the following example. Multiplying and dividing vectors is shown in Example 2.4.

Example 2.3) Practice adding and subtracting different combinations of vectors from Examples 2.1 and 2.2.

$Z_{1}=j 2 \quad Z_{2}=1.6<\pi / 6 \quad Z_{3}=-3-j 4$

- We will need to first convert $Z_{2}$ to rectangular form: $Z_{2}=1.6 \angle \pi / 6 \rightarrow Z_{2}=1.386+j 0.8$

Addition Method $\rightarrow\left(X_{1}+j Y_{1}\right)+\left(X_{2}+j Y_{2}\right)=\left(X_{1}+X_{2}\right)+j\left(Y_{1}+Y_{2}\right)$

- $\quad Z_{1}+Z_{2}=(0+j 2)+(1.386+j 0.8)=(0+1.386)+j(2+0.8)=\underline{1.386+j 2.8}$

- $Z_{1}+Z_{3}=(0+j 2)+(-3-j 4)=(0+-3)+j(2-4)=\underline{-3-j 2}$

- $\quad Z_{2}+Z_{3}=(1.386+j 0.8)+(-3-j 4)=(1.386+-3)+j(0.8-4)=\underline{-1.614-j 3.2}$

Subtraction Method $\rightarrow\left(X_{1}+j Y_{1}\right)-\left(X_{2}+j Y_{2}\right)=\left(X_{1}-X_{2}\right)+j\left(Y_{1}-Y_{2}\right)$

- $\quad Z_{1}-Z_{2}=(0+j 2)-(1.386+j 0.8)=(0-1.386)+j(2-0.8)=\underline{-1.386+j 1.2}$

- $\quad Z_{1}-Z_{3}=(0+j 2)-(-3-j 4)=(0--3)+j(2--4)=\underline{3+j 6}$

- $\quad Z_{2}-Z_{3}=(1.386+j 0.8)-(-3-j 4)=(1.386--3)+j(0.8--4)=\underline{4.1386+j} 4.8$

For addition problems, plotting the vectors can be very helpful in determining if the answer is correct. If you put the second $\left(Z_{2}\right)$ vector on the tail of the first vector $\left(Z_{1}\right)$ the resultant vector $\left(Z_{1}+Z_{2}\right)$ is the vector from the origin $($ point 0,0$)$ to the tail of $Z_{2}$. This is demonstrated in Figure 2.4.

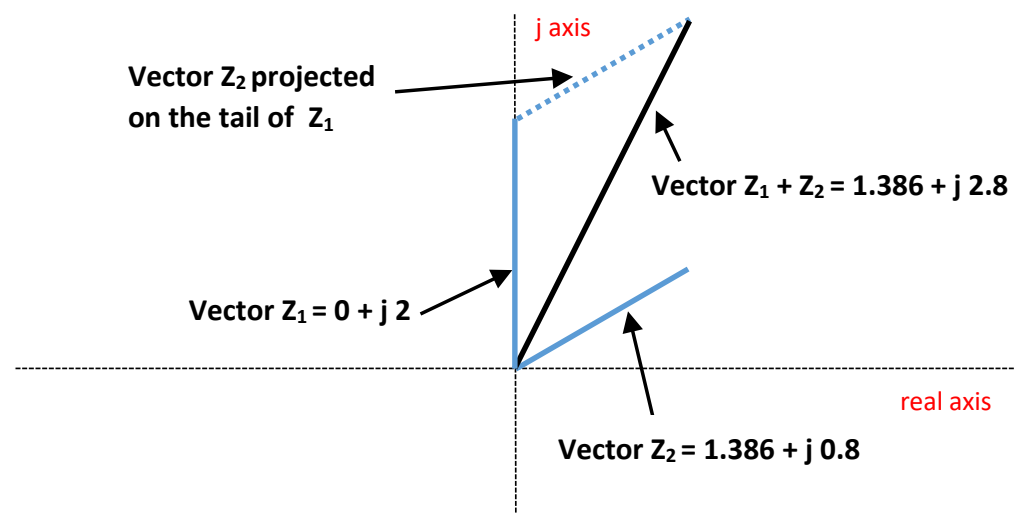

Figure 2.4: Visualization of the addition of two vectors.

The subtraction of vectors is also shown in Example 2.3, but addition of vectors is something that is far more commonly done in AC circuit problems. Since the visualization method shown in Figure 2.4 makes catching math mistakes very easy, it is highly recommended any time vector addition is performed. In fact, a common practice 
in Module 3 will be to determine the total impedance by adding individual impedances and making an impedance plot, which is a plot of all of the impedance vectors in a series circuit.

Example 2.4) Practice multiplying and dividing different combinations of vectors from Example 2.1 and 2.2.

$Z_{1}=j 2 \quad Z_{2}=1.6<\pi / 6 \quad Z_{3}=-3-j 4$

- We will need to first convert $Z_{1}$ and $Z_{3}$ to polar form: $Z_{1}=j 2 \rightarrow \underline{Z_{1}}=\mathbf{2} \angle \pi / 2, Z_{3}=-3-j 4 \rightarrow \underline{Z_{3}}=\mathbf{5} \angle-0.705 \pi$

Multiplication Method $\rightarrow\left[A_{1} \angle \theta_{1}\right] \cdot\left[A_{2} \angle \theta_{2}\right]=\left(A_{1} \cdot A_{2}\right) \angle\left(\theta_{1}+\theta_{2}\right)$

- $\mathrm{Z}_{1} \cdot \mathrm{Z}_{2}=[2 \angle \pi / 2] \cdot[1.6 \angle \pi / 6]=(2 \cdot 1.6) \angle(\pi / 2+\pi / 6)=\underline{3.2 \angle(2 \pi / 3)}$

- $Z_{1} \cdot Z_{3}=[2 \angle \pi / 2] \cdot[5 \angle-0.705 \pi]=(2 \cdot 5) \angle(\pi / 2+-0.705 \pi)=\underline{10} \angle-0.205 \pi$

- $Z_{2} \cdot Z_{3}=[1.6 \angle \pi / 6] \cdot[5 \angle-0.705 \pi]=(1.6 \cdot 5) \angle(\pi / 6+-0.705 \pi)=\underline{8} \angle-0.538 \pi$

Division Method $\rightarrow\left[A_{1} \angle \theta_{1}\right] \div\left[A_{2} \angle \theta_{2}\right]=\left(A_{1} / A_{2}\right) \angle\left(\theta_{1}-\theta_{2}\right)$

- $\mathrm{Z}_{1} \div \mathrm{Z}_{2}=[2 \angle \pi / 2] \div[1.6 \angle \pi / 6]=(2 / 1.6) \angle(\pi / 2-\pi / 6)=\underline{1.25} \angle(\pi / 3)$

- $\mathrm{Z}_{1} \div \mathrm{Z}_{3}=[2 \angle \pi / 2] \div[5 \angle-0.705 \pi]=(2 / 5) \angle(\pi / 2--0.705 \pi)=\underline{\mathbf{0 . 4}} \angle \mathbf{1 . 2 0 5 \pi}=\mathbf{0 . 4} \angle-\mathbf{0 . 7 9 5} \pi$

$>$ Note: $1.205 \pi$ is $>\pi$ so to get the final angle between $-\pi$ and $\pi$ we had to subtract $2 \pi$.

- $\mathrm{Z}_{2} \div \mathrm{Z}_{3}=[1.6 \angle \pi / 6] \div[5 \angle-0.705 \pi]=(1.6 / 5) \angle(\pi / 6--0.705 \pi)=\underline{0.32 \angle 0.8717 \pi}$

Multiplication and division of vectors will be done when Ohm's Law is used in AC circuits. Addition, multiplication, and division will all be used when you combine two impedances in parallel $\rightarrow Z_{\text {Total }}=\left(Z_{1} \cdot Z_{2}\right) /\left(Z_{1}+Z_{2}\right)$

Before ending the discussion on sinusoidal form and how it is represented by a phasor, the concept of the "rotating phasor" is shown in Figure 2.5. This is a good way to see the connection between the phasor $(A \angle \theta)$ and the sinusoid it represents, $A \sin (\omega \cdot t+\theta)$. When time equals zero, $\sin \omega \cdot t$ is also equal to zero and the phasor is located at $A \angle \theta$ as shown in Figure 2.5 .

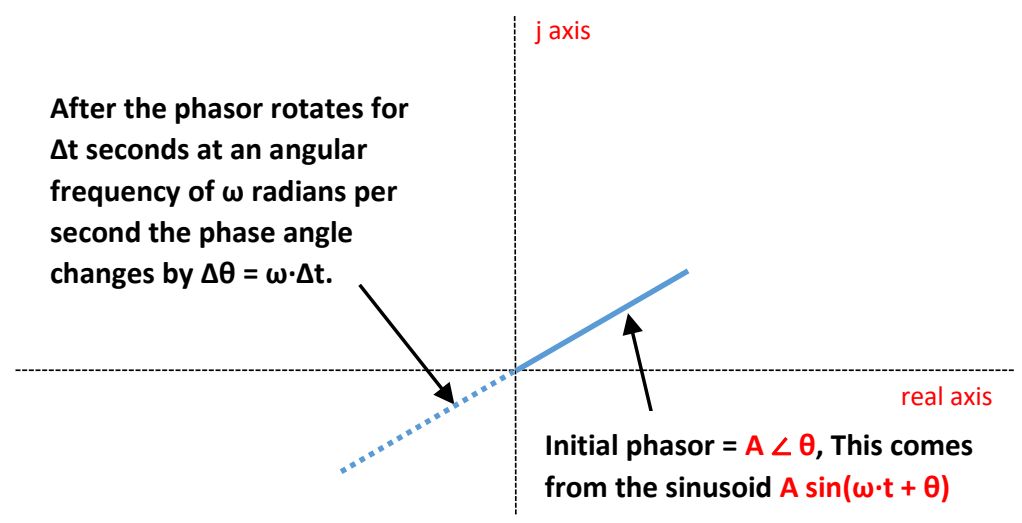

Figure 2.5: Rotating Phasor Visualization.

The phasor (or the vector $A \angle \theta$ ) can be thought of as being rotated by an angular velocity of $\omega$ radians per second. The time variable $t$ in the term $\sin \omega \cdot t$ can be represented by the variable $\Delta t$ because the initial time is zero for the rotating phasor concept. The amplitude $(A)$ of the phasor will not change as it rotates, but the phase $(\theta)$ will change so that the new phase angle is equal to the initial phase and the product of $\omega$ and $\Delta t$.

Example 2.5) If $v(t)=5 \sin (90 \cdot t-0.2 \pi)$, where is the phasor located at after $21.32 \mathrm{~ms}$ ?

- Initially the phasor is located at $5 \angle-0.2 \pi$

- The amount of phase change is $\rightarrow \Delta \theta=\omega \cdot \Delta t=90 \mathrm{rad} / \mathrm{sec}(0.02132 \mathrm{sec})=1.9188$ radians $=0.61 \pi$

- The final phase angle is equal to $\rightarrow \theta=-0.2 \pi+0.61 \pi=0.41 \pi$

- The new location of the phasor is equal to $\rightarrow \underline{5 \angle 0.41 \pi}$

The connection between the phasor and the original sinusoidal function $v(t)$ is as follows:

- If you put in $21.32 \mathrm{~ms}$ into the $\mathrm{v}(\mathrm{t})$ equation you get $\rightarrow 5 \sin (90 \cdot 0.02132-0.2 \pi)=4.8$

- $5 \angle 0.41 \pi$ was the value of the final phasor and $5 \sin (0.41 \pi)=4.8$ 
Example 2.6) If $v(t)=12 \sin (1200 \cdot t+0.8 \pi)$, where is the phasor located at after 3.892 seconds? Make sure the final phasor location is listed as a value between $-\pi$ and $+\pi$ so it can be easily visualized on the real, imaginary $(x, y)$ axis. ALWAYS put phase values between $-\pi$ and $+\pi$ (or $-180^{\circ}$ and $+180^{\circ}$ ).

- Initially the phasor is located at $12 \angle+0.8 \pi$

- The amount of phase change is $\rightarrow \Delta \theta=\omega \cdot \Delta t=1200 \mathrm{rad} / \mathrm{sec}(3.892 \mathrm{sec})=4670.4$ radians $=1486.6345 \pi$

- The final phase angle is equal to $\rightarrow \theta=+0.8 \pi+1486.6345 \pi=1487.4345 \pi \leftarrow$ This is $>\pi$ so subtract multiples of $2 \pi$

- If $2 \pi$ is continually subtracted from $1487.4345 \pi$ until $\theta$ is between $-\pi$ and $\pi$ the value is equal to is at the same location as $0.19 \pi$. This can be found by subtracting $2 \pi$ until $\theta$ is between $-\pi$ and $\pi$ you get $-0.5655 \pi$

- The new location of the phasor is equal to $\rightarrow \underline{12 \angle-0.5655 \pi}$

The following is only done to verify the result:

- If you put in $3.892 \mathrm{~s}$ into the $\mathrm{v}(\mathrm{t})$ equation you get $\rightarrow 12 \sin (1200 \cdot 3.892+0.8 \pi)=-11.74678$

- $5 \angle 0.41 \pi$ was the value of the final phasor and $12 \sin (-0.5655 \pi)=-11.74678$

ALWAYS put phase values between $-\pi$ and $+\pi$ (or $-180^{\circ}$ and $+180^{\circ}$ ) 


\section{Module 3 - RLC Circuit Analysis with AC Sources}

Module 3 is broken up into the following 5 sections.

- Section 3.1 - Introduction of Impedance and Admittance and the Laplace Transform

- Section 3.2-Series RLC Circuits

- Section 3.3-Parallel RLC Circuits

- Section 3.4-AC Power Calculations

- Section 3.5 - AC Maximum Power Transfer

\section{Section 3.1 - Introduction of Impedance and Admittance and the Laplace Transform}

Before solving RLC circuits with AC sources there is some terminology that needs to be discussed. First of all this eBook focuses only on RLC circuits with AC sources. For brevity, these types of circuits will be referred to as "AC Circuits". For DC circuits at steady state, resistors were the only components used in calculations because all capacitors in the circuit were replaced with open circuits and all inductors in the circuit were replaced with short circuits. For AC circuits at steady state the capacitors/inductors no longer can be replaced with open/short circuits, but instead are included in the calculations. The remainder of this module will look only at AC circuits at steady state. Transient analysis of AC circuits is accomplished by using Laplace Transform solving techniques. Some Laplace Transform examples are shown in the Appendix.

The impedance, Z, is shown for resistors, capacitors, and inductors in the left side of Table 3.1 in both rectangular and polar (Phasor) form. The magnitude of the impedance is called the reactance, X. For AC circuits in series the impedance of the components can be added together to get the total impedance, just like the resistors where added together to get the total resistance in DC circuits. For networks of components in parallel and series the math for combining them because very time consuming since for each combination a complex algebra problem must be performed. For this reason only series (section 3.2) and parallel (section 3.3) AC circuits will be discussed in this eBook. However, the same rules for combining resistors are used for combining impedances as shown in the DC and $\mathrm{AC}$ equation comparison below:

\section{Equations Used in DC Circuits}

- N Resistors in series: $\mathrm{R}_{\text {tot }}=R_{1}+R_{2}+\cdots R_{N}$

-2 Resistors in parallel: $R_{t o t}=\frac{R_{1} \cdot R_{2}}{R_{1}+R_{2}}$

-N Resistors in parallel: $R_{\text {tot }}=\frac{1}{\frac{1}{R_{1}}+\frac{1}{R_{2}}+\cdots \frac{1}{R_{N}}}$

- Conductance: $G=\frac{1}{R}$

-N Resistors in parallel: $G_{\text {tot }}=G_{1}+G_{2}+\cdots G_{N}$

-Ohm's Law: V $=I \cdot R$ or $I=\frac{V}{R}=V \cdot G$

\section{Equations Used in AC Circuits}

- R, L, and Cs in series: $Z_{t o t}=Z_{1}+Z_{2}+\cdots Z_{N}$

- 2 Impedances in parallel: $Z_{\text {tot }}=\frac{Z_{1} \cdot Z_{2}}{Z_{1}+Z_{2}}$

- R, L, and Cs in parallel: $Z_{\text {tot }}=\frac{1}{\frac{1}{Z_{1}}+\frac{1}{Z_{2}}+\cdots \frac{1}{Z_{N}}}$

- Admittance: $Y=\frac{1}{Z}$

- R, L, and Cs in parallel: $Y_{\text {tot }}=Y_{1}+Y_{2}+\cdots Y_{N}$

- Ohm's Law: $V=I \cdot Z$ or $I=\frac{V}{Z}=V \cdot Y$ 
Table 3.1) Impedance, Reactance, Admittance, and Susceptance for Resistors, Capacitors, and Inductors

\begin{tabular}{|c|c|c|c|c|c|c|c|c|}
\hline \multirow[b]{3}{*}{ Component } & \multicolumn{3}{|c|}{ Impedance $=\mathrm{Z}=\mathrm{R}+\mathrm{j} X$ (Units $=\Omega$ ) } & \multirow{3}{*}{$\begin{array}{c}\text { Reactance } \\
\text { Variable: } \mathrm{X} \\
\text { Units }=\Omega\end{array}$} & \multicolumn{3}{|c|}{ Admittance $=Y=G+j B$ (Units $=$ Siemens) } & \multirow{3}{*}{$\begin{array}{c}\text { Susceptance } \\
\text { Variable: B } \\
\text { Units (Siemens) }\end{array}$} \\
\hline & \multirow{2}{*}{$\begin{array}{c}\text { Laplace } \\
\text { Form }\end{array}$} & \multicolumn{2}{|c|}{ Vector Form } & & \multirow{2}{*}{$\begin{array}{c}\text { Laplace } \\
\text { Form } \\
\end{array}$} & \multicolumn{2}{|c|}{ Vector Form } & \\
\hline & & $X+j Y$ & $A<\theta$ & & & $X+j Y$ & $A<\theta$ & \\
\hline Resistor & $\mathrm{R}$ & $Z_{R}=R$ & $\mathrm{R} \angle 0$ & 0 & G & $Y_{R}=G=1 / R$ & $\mathrm{G} \angle 0$ & 0 \\
\hline Inductor & Ls & $Z_{L}=j L \omega$ & $\mathrm{L} \omega \angle 90^{\circ}$ & $X_{L}=L \omega$ & $1 /($ Ls) & $Y_{L}=-j /(L \omega)$ & $1 /(L \omega)<-90^{\circ}$ & $B_{L}=1 /(L \omega)$ \\
\hline Capacitor & $1 /(C s)$ & $Z_{C}=-j / C \omega$ & $1 /(C \omega)<-90^{\circ}$ & $X_{C}=1 /(C \omega)$ & Cs & $Y_{C}=j C \omega$ & $\mathrm{C} \omega \angle 90^{\circ}$ & $\mathrm{B}_{\mathrm{C}}=\mathrm{C} \omega$ \\
\hline
\end{tabular}

\section{Section 3.2 - Series RLC Circuits}

For DC circuits with series resistors all of the resistors are added to determine the total resistance. Then the current in the loop is calculated from Ohm's Law $(I=V s /$ Rtot $)$. For AC circuits the same process is followed except resistance is changed to impedance and each of the variables is a phasor instead of a scalar. The next three examples show the process.

Example 3.1) Verify the output ( $\left.V_{\text {out }}\right)$ for the circuit in Figure 3.1 by finding the total impedance and using Ohm's Law. Go to Figure 3.2 to see the oscilloscope plot of the waveforms

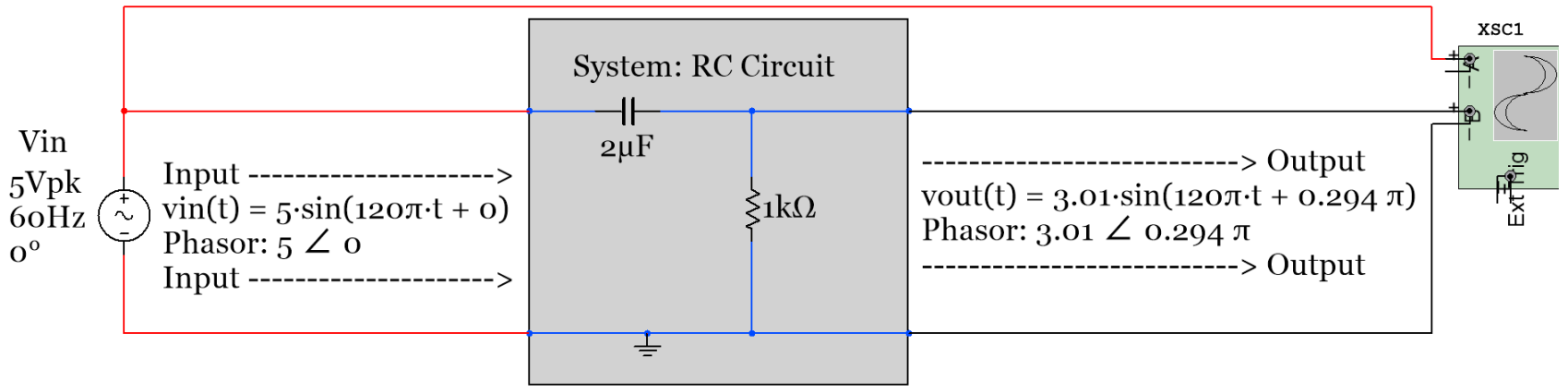

$$
\begin{aligned}
& \text { Ztotal }=\mathrm{Zt}=\mathrm{R}+\mathrm{Zc}=\mathrm{R}-\mathrm{j}(1 / \mathrm{C} \omega) \\
& \mathrm{Zt}=1000-\mathrm{j} /\left(2^{*} \mathrm{pi}^{*} 6 \mathrm{O}^{*} 2 \mathrm{e}-6\right)=1000-\mathrm{j} 1326.3 \\
& \mathrm{Zt}=1661 \angle-53^{0} \\
& \mathrm{I}=\mathrm{Vin} / \mathrm{Zt}=(5 \angle \mathrm{o}) /\left(1661 \angle-53^{0}\right)=3.01 \angle 53^{0} \mathrm{~mA} \\
& \text { Vout }=3.01 \angle 53^{0}=3.01 \angle 0.294 \pi \text { radians } \\
& \text { vout(t) }=3.01 \cdot \sin (120 \pi \cdot \mathrm{t}+0.294 \pi)
\end{aligned}
$$

Figure 3.1: Solving for the output in phasor and sinusoidal form for Example 3.1.

For this example the voltage across the capacitor could also be solved for by using Ohm's Law.

- $\left.\mathrm{V}_{\mathrm{C}}=1 \cdot \mathrm{Z}_{\mathrm{C}}=1 \cdot \mathrm{X}_{C} \angle-90^{0}=1 \cdot(C \cdot \omega)^{-1} \angle-90^{0}=\left(3.01 \angle 53^{\circ}\right) \cdot[(2 \mathrm{e}-6) \cdot(2 \cdot \pi \cdot 60)]^{-1} \angle-90^{\circ}\right)=4 \angle-37^{0}=\underline{\mathbf{4}} \angle-0.20556 \pi$

- $v_{c}(t)=4 \sin (120 \pi t-0.20556 \pi)$

Example 3.1 shows the process of solving series AC circuits, but one thing that isn't addressed is the leading and lagging aspects that were discussed in Section 1.2.

Recall: A waveform is leading another waveform if it completes its sinusoidal cycle first. Mathematically, a waveform leads another waveform if it has a larger phase. Since the phase of $V_{\text {out }}\left(\theta=53^{\circ}\right)$ is greater than $V_{\text {in }}(\theta$ $=0^{\circ}$ ) in Example $3.1 \mathrm{~V}_{\text {out }}$ is leading. 


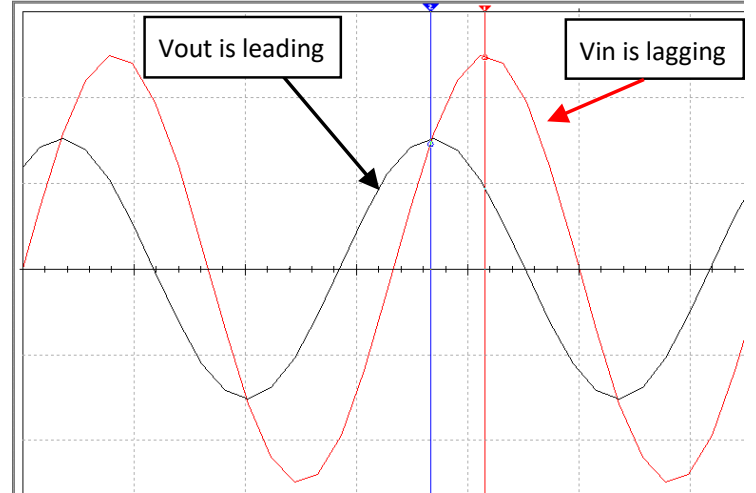

Figure 3.2: Scope plot of the circuit in Example 3.1

The leading waveform will be the one with the largest phase, but there is a pneumonic called "ELI the ICE man" that can be used to determine which waveform is leading or lagging without calculating the phase.

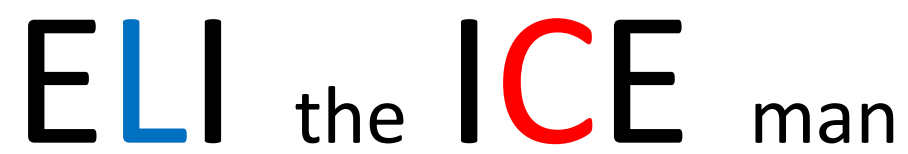

The $L$ and the $C$ stand for inductor and capacitor, respectively (I will frequently use Blue font for inductors and red font for capacitors). If the circuit is a series RL circuit it is an ELI circuit and if it is a series RC circuit it is an ICE circuit. If the circuit is a series RLC circuit, then the quadrant of the total impedance will determine whether it is an ELI or ICE circuit. If Ztotal is in quadrant I it is an ELI circuit and if it is in quadrant IV it is an ICE circuit. The "E" stands for the potential, which is the input voltage. The "I" stands for the current in the single loop AC circuit. Since the current and voltage across a resistor are always in phase with each other in a series AC circuit then the current (I) can also be used to represent the voltage across a resistor for lead/lag determination. If " $E$ " is written to the left of "I" (i.e. ELI) then "E" (or Vin) is "first" and leads "I" (the current or voltage across the resistor). The opposite is true for ICE circuits. In summary:

- $\quad$ ELI - Any RL circuit or an RLC series AC circuit with a total impedance in quadrant I.

- The input voltage $\left(\mathbf{V}_{\text {in }}\right)$ leads the current $(\mathbf{I})$ or the voltage across any resistor $\left(\mathbf{V}_{\mathbf{R}}\right)$ in an ELI circuit.

- ICE - Any RC circuit or an RLC series AC circuit with a total impedance in quadrant IV.

- The input voltage $\left(\mathbf{V}_{\text {in }}\right)$ lags the current $(\mathbf{I})$ or the voltage across any resistor $\left(\mathbf{V}_{\mathbf{R}}\right)$ in an ICE circuit. 


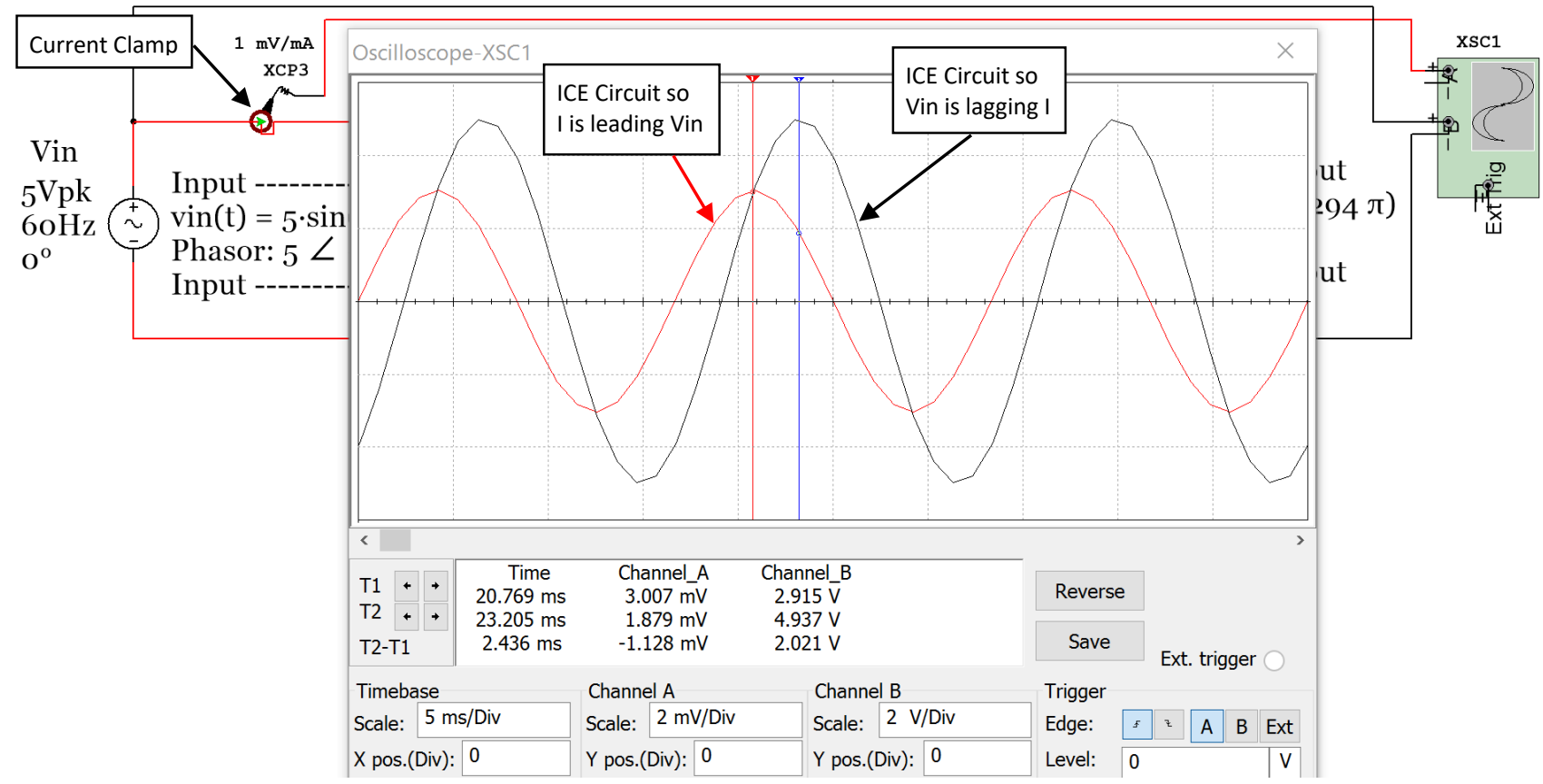

Figure 3.3: Input Voltage a Current scope plot of circuit in Example 3.1.

For the circuit in example 3.1 the input voltage and current are plotted in Figure 3.3. The current can be plotted on a scope using the current clamp as shown in Figure 3.3. The current clamp conversion is shown in this example to be $1 \mathrm{mV} / \mathrm{mA}$ so the peak current reading of the red current waveform is $3.007 \mathrm{mV}$, which equates to 3.007 mA. The next example is an RLC cirucit. Notice how in the calculations in Figure 3.4 that the current and $V_{R}$ both have a phase angle of $-18.5^{\circ}$. This means they are "in phase" with each other.

Example 3.2) Solve the circuit in Figure 3.4 for VR1 and verify it with the Multisim oscilloscope.

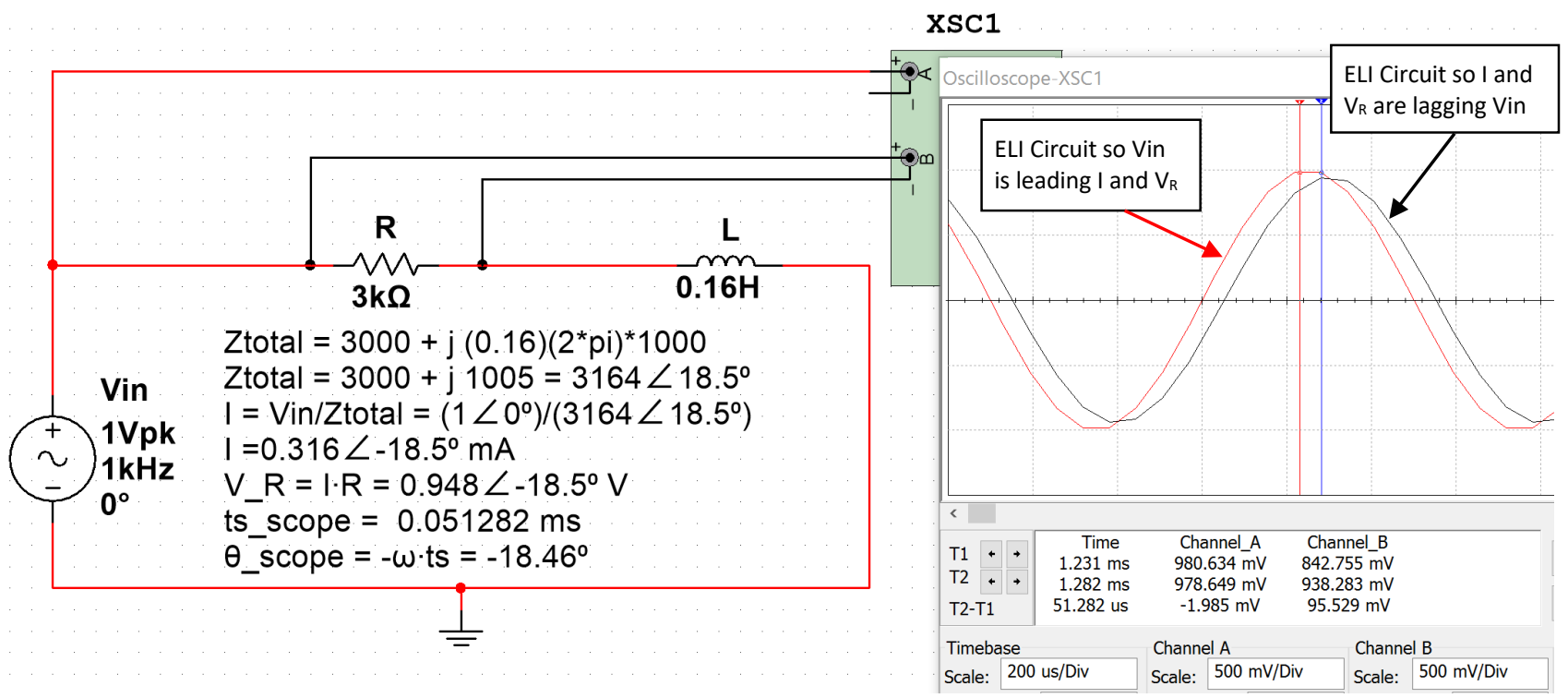

Figure 3.4: Circuit and Scope plot for Example 3.2. $V_{\text {in }}$ is the Red sinusoid and $V_{R}$ is the black sinusoid. 
The calculations for Example 3.2 are shown in Figure 3.4. The following link can be used to verify the total impedance calculation in a RLC circuit: http://hyperphysics.phy-astr.gsu.edu/hbase/electric/powerac.html

The phase of $V_{R 1}$ that was calculated using the time shift measurement from the scope is equal to $-18.46^{\circ}$ and the value was calculated as $-18.5^{\circ}$. $\mathrm{V}_{\text {in_pk }}$ should be equal to $1 \mathrm{~V}$, but is measured as $980.634 \mathrm{mV}$ and $\mathrm{V}_{\mathrm{R} 1 \_p k}$ should be equal to $948 \mathrm{mV}$, but it is measured as $938.284 \mathrm{mV}$ in Multisim. The small errors of the time shift and peak voltage measurements are expected due to limited resolution of the Multisim oscilloscope.

Note: If the minus sign of the Multisim oscilloscope is left disconnected then it is automatically assumed to be ground. Therefore, only the + side of channel A is connected in Figure 3.4. If you want to take a scope measurement across a component that is not connected to ground (i.e. $v_{R}$ in Figure 3.4) then you connect the two sides of the component to the + and - side of the scope channel. Most oscilloscopes in the real world require that the minus sign (or black clip) be connected to ground. For example, Figure 3.6 shows a sinusoid that is connected to channel 1 of a Rigol DS1074 oscilloscope. This scope has 4 channels. Each channel has a probe that has a black clip that can only be connected to ground as shown in Figure 3.5. If you want to take a measurement across a component that is NOT connected to ground (i.e. differential measurement) then you have to connect 2 different probes to the two sides of the component and use the Math subtraction function to determine the voltage across the component.

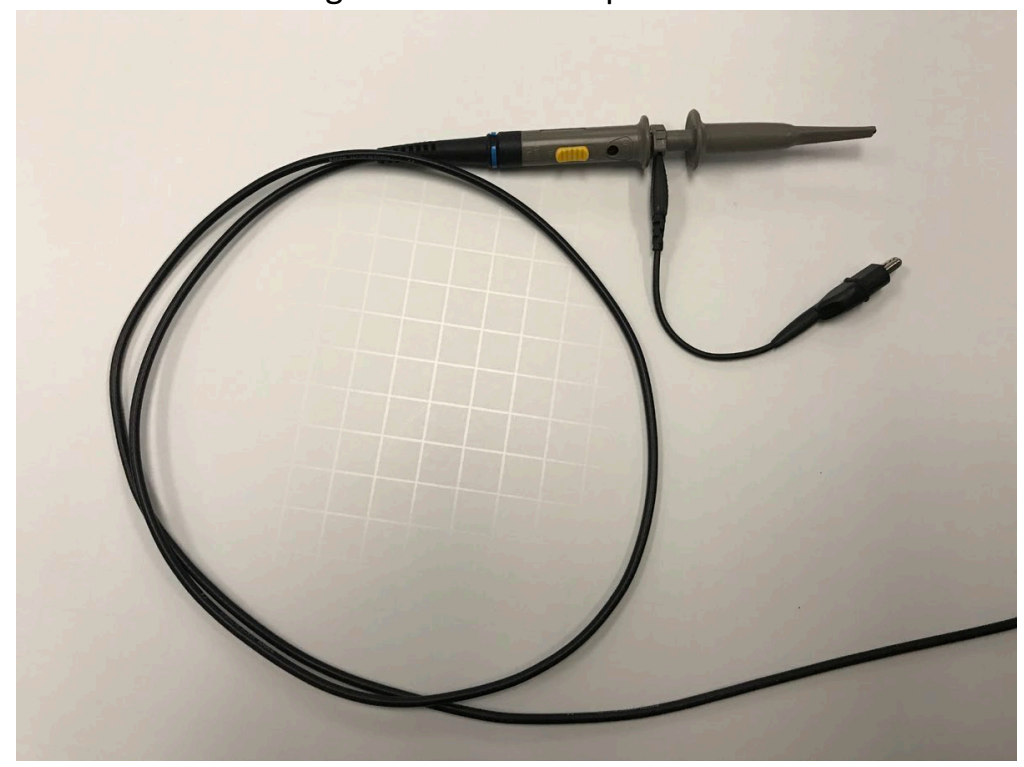

Figure 3.5: Rigol Oscilloscope Probe: http://www.prc68.com///RigolDS1052E.shtml 


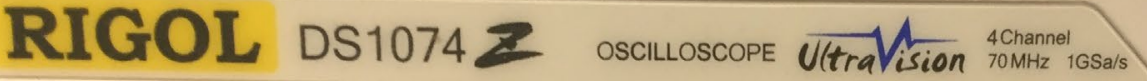

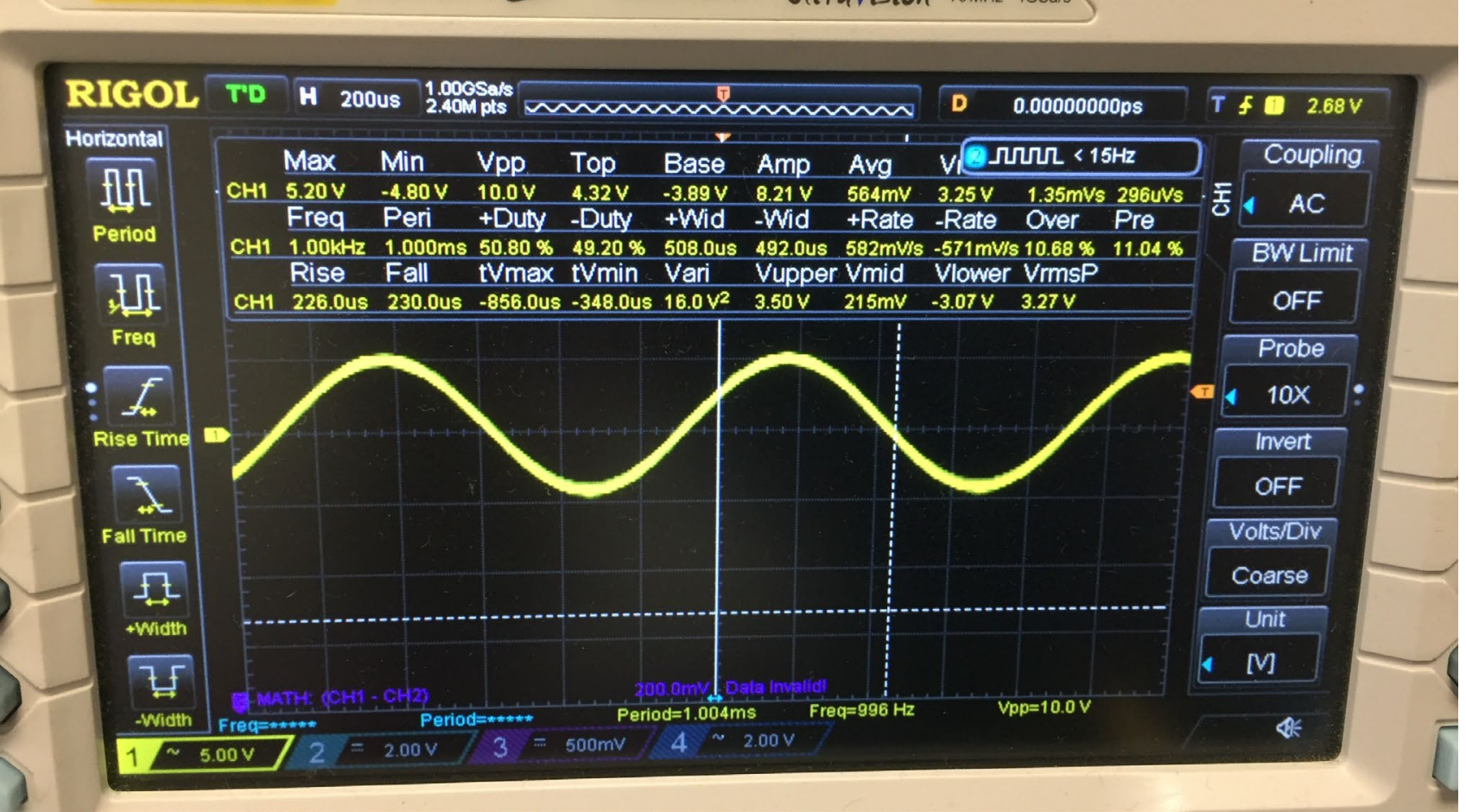

Figure 3.6: Photo of Rigol DS1074 Oscilloscope with a 10Vp-p sine wave at $1 \mathrm{kHz}$.

Example 3.3) Solve the circuit in Example 3.2 for the voltage across the inductor and verify it in Multisim.

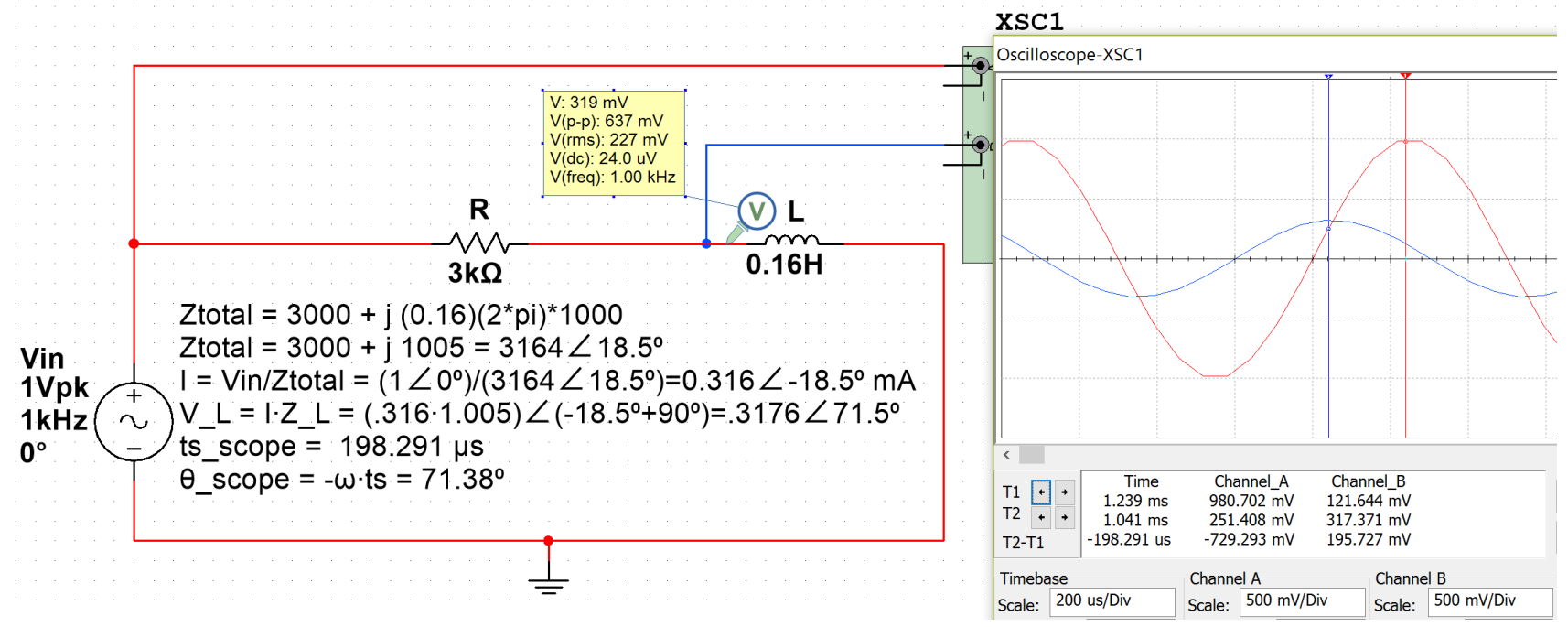

Figure 3.7: Circuit and Scope plot for Example 3.3. $V_{\text {in }}$ is the Red sinusoid and $V_{L}$ is the blue sinusoid.

Calculation and simulation results comparison for Example 3.3

- $\mathrm{V}_{\mathrm{L}}=0.3176 \angle 71.5^{\circ}$ (Calculated), $\mathrm{V}_{\mathrm{L}}$ Phase Measurement from scope $=71.38^{\circ}$

- $V_{\mathrm{L}}$ Amplitude Measurement: $\mathrm{V}_{\mathrm{L}}=\mathrm{V}_{\mathrm{PP}} / 2=637 \mathrm{mV} / 2=0.3185 \mathrm{~V}$ (Probe) or $0.317371 \mathrm{~V}$ (Scope)

- Notice that $\mathbf{V}_{\mathbf{L}}$ leads $\mathbf{V}_{\text {in }}$ in the scope plot, but this cannot be determined from the ELI the ICE man pneumonic that only relates $V_{\text {in }}$ and I (or $V_{R}$ ). $V_{L}$ has larger phase that $V_{\text {in }}$ so it leads $V_{\text {in }}$. 
The last series AC circuit example will be a RLC circuit. For this example the impedance plot will be drawn to help determine the phasor of the total impedance that will result when all impedances are added. Even though only one resistor, one capacitor, and one inductor are in this circuit there could be multiple components in series and the same process of summing all impedances would still be performed in the same way it is done in Example 3.4.

Example 3.4) Solve for the total impedance phasor $\left(Z_{T}\right)$ in the circuit in Figure 3.8 when $L=0.2 H$ and when $L=1.8$ H.

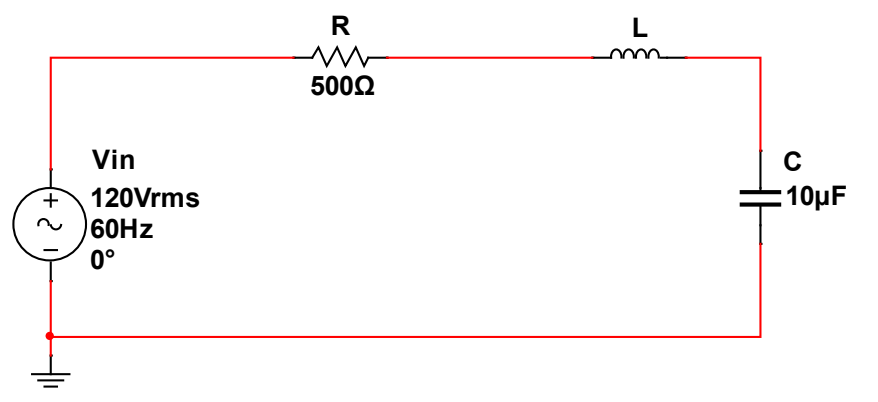

$$
\begin{aligned}
& V_{\text {in_pk }}=120 \mathrm{~V}_{\text {in_rms }} \cdot \sqrt{2}=169.7 \mathrm{~V} \\
& V_{\text {in }}=169.7 \angle 0^{0} \\
& Z_{\text {tot }}=R+\mathrm{j} \cdot \omega-\mathrm{j} /(\mathrm{C} \cdot \omega) \\
& Z_{\text {tot }}=500+\mathrm{j}(\mathrm{L} \cdot 120 \pi)-\mathrm{j} /(10 \mathrm{e}-6 \cdot 120 \pi) \\
& Z_{\text {tot }}=500-\mathrm{j} 189.86=534.8 \angle-20.8^{0}(\mathrm{~L}=0.2 \mathrm{H}) \\
& Z_{\text {tot }}=500+\mathrm{j} 413.33=648.7 \angle 39.6^{0}(\mathrm{~L}=1.8 \mathrm{H})
\end{aligned}
$$

\section{Figure 3.8: Circuit for Example3.4}

The phasors for the total impedance $\left(Z_{\text {tot }}\right)$ for each of the two cases $(L=0.2 \mathrm{H}$ and $\mathrm{L}=1.8 \mathrm{H})$ are calculated in textbox in Figure 3.8. An impedance plot is performed in Figure 3.9 to help verify the total impedance was calculated correctly.

- Case 1 where $\mathrm{L}=0.2 \mathrm{H}$ results in $\mathrm{Z}_{\text {tot }}$ in quadrant IV, which means it is an ICE circuit and $\mathrm{V}_{\text {in }}$ lags the current and the voltage across the resistor.

- Case 2 where $L=1.8 \mathrm{H}$ results in $Z_{\text {tot }}$ in quadrant $\mathrm{I}$, which means it is an ELI circuit and $V_{\text {in }}$ leads the current and the voltage across the resistor.
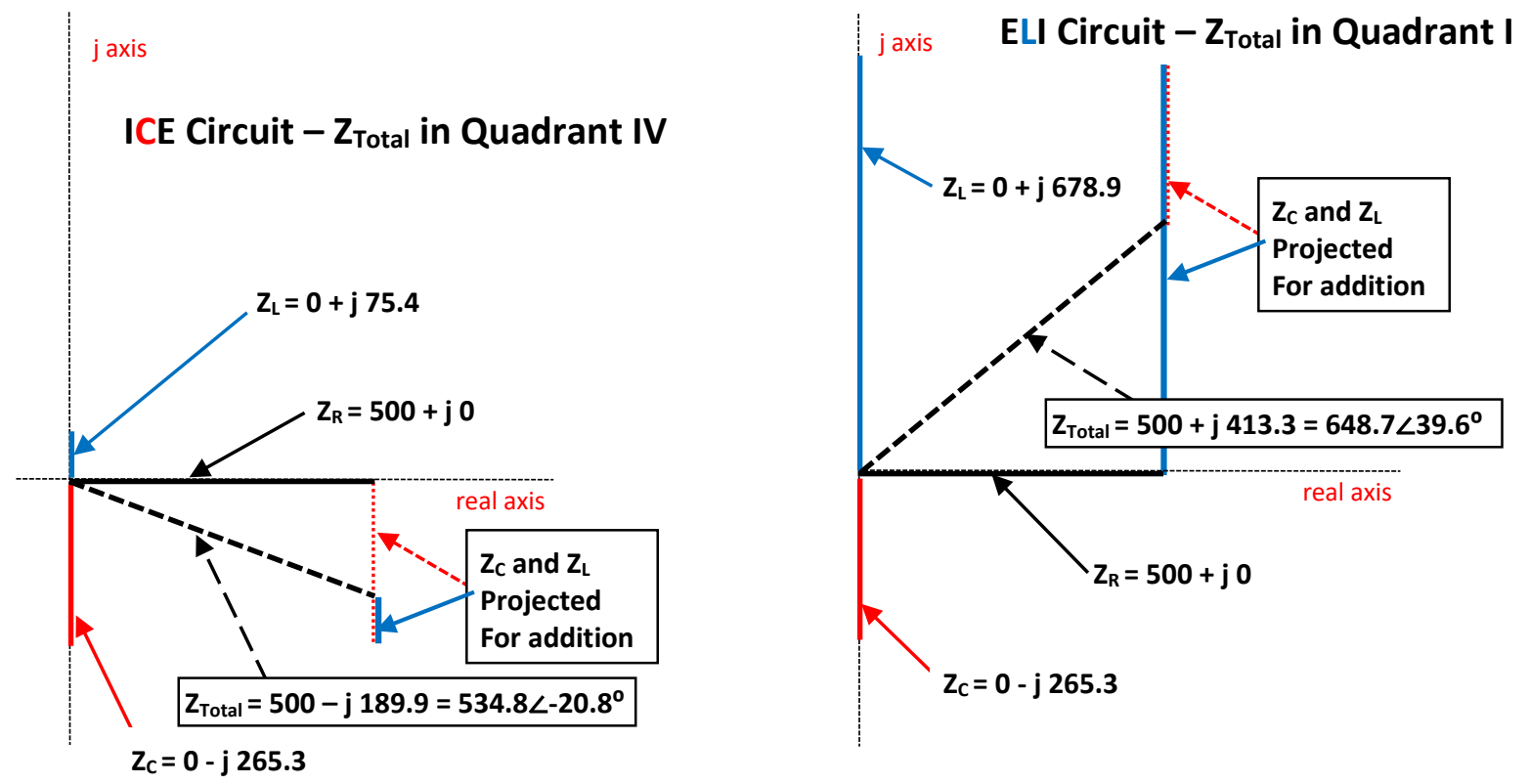

Figure 3.9: Impedance Plots for Example3.4. Case 1: $L=0.2 \mathrm{H}$ (Left Plot), Case 2: $\mathrm{L}=1.8 \mathrm{H}$ (Right Plot) 
Example 3.5) Solve for the loop current and $V_{R}$ for each of the two cases in Example $3.4(L=0.2 \mathrm{H}$ and $L=1.8 \mathrm{H})$ and represent them in phasor form and sinusoidal form. Verify the loop current results with Multisim.

First, the current will be calculated using Ohm's Law and the Multisim values will be measured on the scope.

\begin{tabular}{|c|c|}
\hline * Case 1: $\mathrm{L}=0.2 \mathrm{H}, \mathrm{Z}_{\text {Total }}=534.8 \angle-20.8^{\circ}$ (see Fig. 3.9) & * Case 2: $\mathrm{L}=1.8 \mathrm{H}, \mathrm{Z}_{\text {Total }}=648.7 \angle 39.6^{\circ}$ (see Fig. 3.9) \\
\hline $\begin{array}{l}* I=\frac{169.7 \angle 0^{0}}{534.8 \angle-20.8^{0}}=0.3173 \angle 20.8^{0}=0.3173 \angle 0.116 \pi \\
* V_{R}=I \cdot R=(0.3173 \angle 0.116 \pi) \cdot 500 \Omega=158.7 \angle 0.116 \pi \\
* V_{\mathrm{R}}(\text { Multisim Probe })=113 \mathrm{Vrms} * \sqrt{2}=159.8 \mathrm{~V} \\
* \mathrm{i}(\mathrm{t})=0.3173 \sin (120 \pi \mathrm{t}+0.116 \pi) \\
* V_{\mathrm{R}}(\mathrm{t})=158.7 \sin (120 \pi \mathrm{t}+0.116 \pi)\end{array}$ & $\begin{array}{l}* I=\frac{169.7 \angle 0^{0}}{648.7 \angle 39.6^{0}}=0.2616 \angle-39.6^{0}=0.2616 \angle-0.22 \pi \\
* V_{R}=I \cdot R=(0.2616 \angle-0.22 \pi) \cdot 500 \Omega=130.8 \angle-0.22 \pi \\
* V_{\mathrm{R}}(\text { Multisim Probe })=91.4 \mathrm{Vrms} * \sqrt{2}=129.6 \mathrm{~V} \\
* \mathrm{i}(\mathrm{t})=0.2616 \sin (120 \pi \mathrm{t}-0.22 \pi) \\
* \mathrm{~V}_{\mathrm{R}}(\mathrm{t})=130.8 \sin (120 \pi \mathrm{t}-0.22 \pi)\end{array}$ \\
\hline \multicolumn{2}{|c|}{ * Multisim Current Verification for Case 1 and Case 2 current calculations (See Figure 3.10 below). } \\
\hline * Multisim Amplitude, $\mathrm{A}=312 \mathrm{mV}$ & * Multisim Amplitude, $A=257 \mathrm{mV}$ \\
\hline$*$ Multisim Time Shift, $t_{\mathrm{s}}=-0.94 \mathrm{~ms}$ & $*$ Multisim Time Shift, $\mathrm{t}_{\mathrm{s}}=1.833 \mathrm{~ms}$ \\
\hline * Phase Shift, $\theta=-t_{s^{\prime}} \omega=x y z \cdot 120 \cdot \pi=20.3^{\circ}$ & $*$ Multisim Phase Shift, $\theta=-t_{s} \cdot \omega=-39.7^{0}$ \\
\hline
\end{tabular}

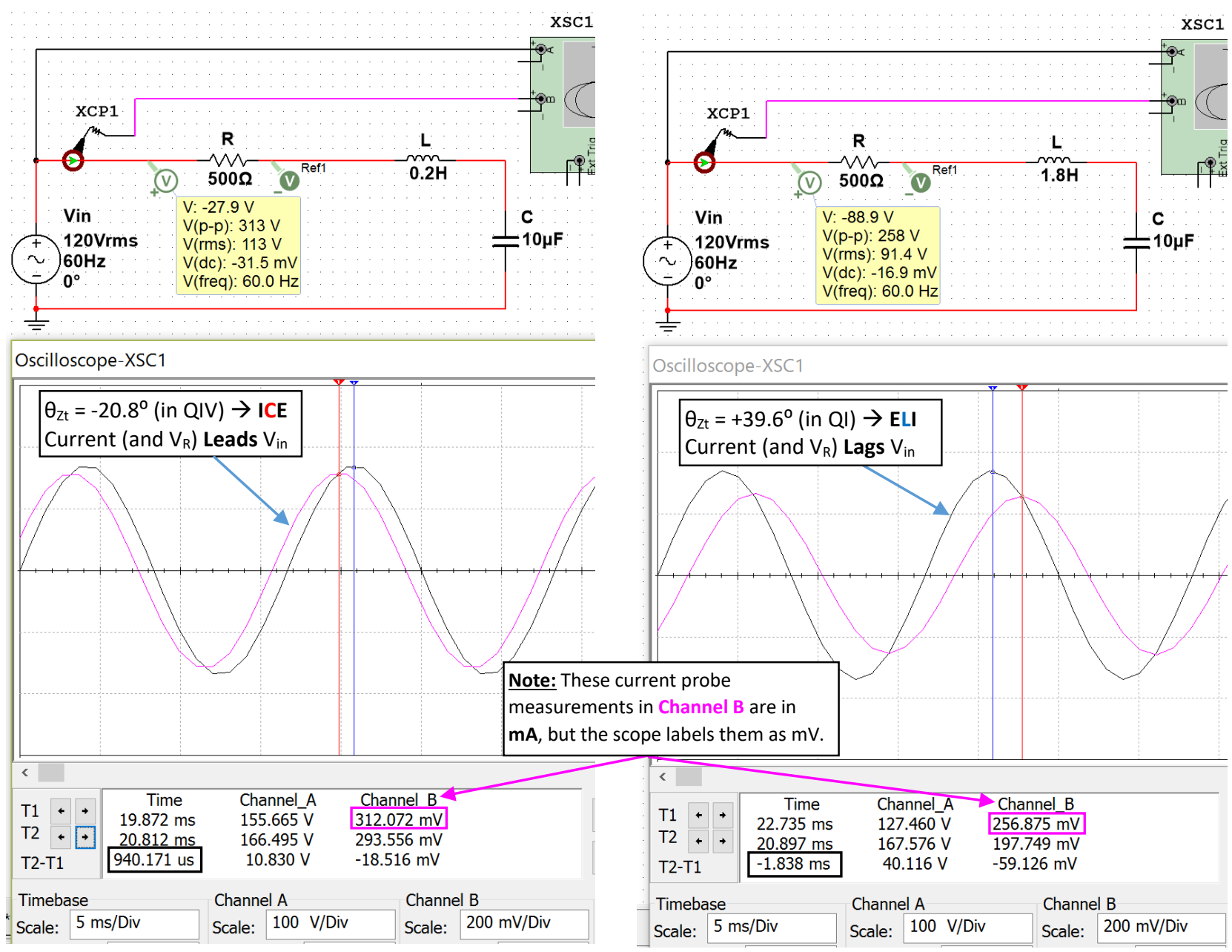

Figure 3.10: Current (Pink) and $V_{\text {in }}$ (Black) Plots for Case 1: $L=0.2 \mathrm{H}$ (Left) and Case 2: $L=1.8 \mathrm{H}$ (Right) 
Example 3.6) Solve for the inductor and capacitor voltage for the two cases in Example 3.4. Case 1: $\underline{L}=\mathbf{0 . 2} \boldsymbol{H}$ and Case 2: $L=1.8 \mathrm{H}$. Represent voltages in phasor form and sinusoidal form. Verify the Case 1 values in Multisim.

$\mathrm{V}_{\mathrm{L}}$ (peak voltage) will be solved two different ways: 1) Ohm's Law and 2) Voltage Divider Rule (VDR).

* Case 1: $\mathrm{Z}_{\text {Total }}=534.8 \angle-20.8^{\circ}$ and $\mathrm{I}=0.3173 \angle 20.8^{\circ} \quad *$ Case 2: $\mathrm{Z}_{\text {Total }}=648.7 \angle 39.6^{\circ}$ and $\mathrm{I}=0.2616 \angle-39.6^{\circ}$

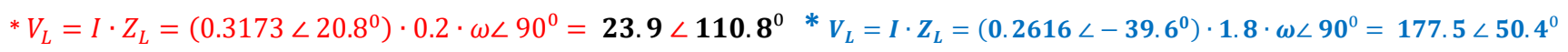

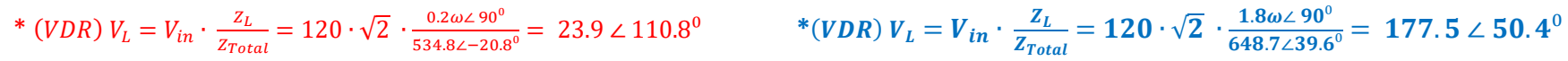

$* V_{L}(t)=23.9 \sin (120 \pi t+0.616 \pi) \quad$ Note: $0.616 \pi=110.8^{\circ} * V_{L}(t)=177.5 \sin (120 \pi t+0.28 \pi)$ Note: $0.28 \pi=50.4^{\circ}$

* Multisim Case 1: $V_{L}=\mathbf{2 3 . 8} \mathbf{V}$ (scope), $V_{L}=17.2 \mathrm{Vrms} \cdot \sqrt{2}=\mathbf{2 4 . 3} \mathbf{V}$ (probe), $\mathrm{t}_{\mathrm{S}}=-5.128 \mathrm{~ms}, \theta=-\mathrm{t}_{\mathrm{s}} \cdot \mathrm{\omega}=\mathbf{1 1 0 . \mathbf { 8 } ^ { 0 }}$

${ }^{*} V_{C}=I \cdot Z_{C}=\left(0.3173 \angle 20.8^{0}\right) \cdot \frac{1}{\left(10^{-6}\right) \cdot \omega} \angle-90^{0}=\mathbf{8 4 . 2} \angle-\mathbf{6 9 . 2}^{0} \quad * V_{C}=I \cdot Z_{C}=\left(.2616 \angle-39.6^{0}\right) \cdot \frac{1}{10^{-6} \omega} \angle-90^{0}=69.4 \angle 129.6^{0}$

$* v_{c}(t)=84.2 \sin (120 \pi t-\mathbf{0 . 3 8 4 \pi}) \quad$ Note: $-0.384 \pi=-69.2^{\circ} \quad * v_{c}(t)=69.4 \sin (120 \pi t+0.72 \pi) \quad$ Note: $0.72 \pi=129.6^{\circ}$

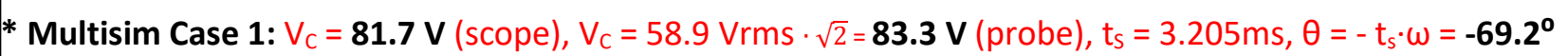
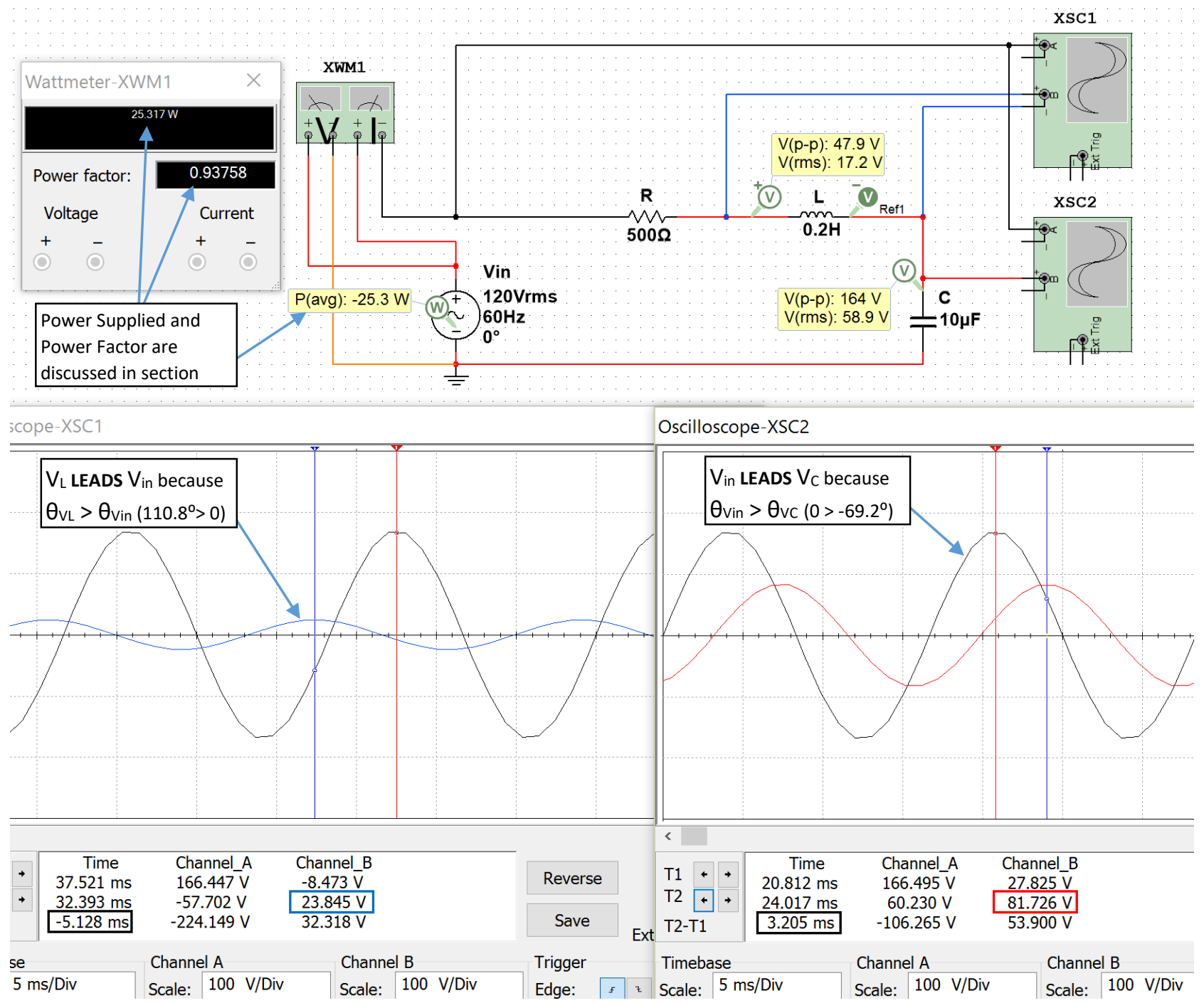

Figure 3.11: Case 1 in Example 3.6, $V_{\text {in }}$ (Black) \& $V_{L}$ (Blue) (Left Plot), $V_{\text {in }}$ (Black) \& $V_{C}($ Red) (Right Plot) 
The process involved in solving RLC series circuits is summarized in Table 3.2.

Table 3.2) Summary of the process used to solve for all parameters in a series RLC circuit.

\begin{tabular}{|l|}
\hline Process for solving RLC Series Circuits \\
\hline 1) Add all impedances to get the total impedance (Ztotal) \\
* The magnitude of the inductor's impedance is the Inductive Reactance $\left(X_{L}\right)$ \\
* The magnitude of the capacitor's impedance is the Capacitive Reactance $\left(X_{C}\right)$ \\
\hline 2) Convert to Phasor form: Input voltage $=V_{\text {in_pk }} \angle \theta$ and Total Impedance $=A_{Z t} \angle \theta_{Z t}$ \\
* If $\theta_{Z t}>0$ it is an ELI circuit and Vin LEADS I and $V_{R}$ \\
* If $\theta_{Z t}<0$ it is an ICE circuit and Vin LAGS I and $V_{R}$ \\
\hline 3) Calculate the Current using I = Vin/Ztotal \\
\hline 4) Find voltage phasors using Ohm's Law: $V_{R}=I \cdot R, V_{L}=I \cdot Z_{L}, V_{C}=I \cdot Z_{C}$ \\
* When comparing 2 signals, the one with the largest phase LEADS. \\
\hline 5) Write the series circuit's loop current and all voltages in sinusoidal form: A.sin $(\boldsymbol{\omega} \cdot \mathbf{t}+\boldsymbol{\theta})$ \\
\hline
\end{tabular}

\section{Section 3.3 - Parallel RLC Circuits}

In this section a slight modification of the process in Table 3.2 is used to solve parallel RLC circuits.

Table 3.3) Summary of the process used to solve for all parameters in a parallel RLC circuit.

\begin{tabular}{|c|}
\hline rocess for solving RLC Parallel Circuits \\
\hline 1) Add all admittances to get the total admittance (Ytotal) \\
\hline * The magnitude of the inductor's admittance is the Inductive Susceptance $\left(B_{L}\right)$ \\
\hline * The magnitude of the capacitor's admittance is the Capacitive Susceptance ( $\left.B_{c}\right)$ \\
\hline 2) Convert to Phasor form: Input voltage $=\mathrm{V}_{\text {in } \_p k} \angle \theta$ and Total Admittance $=A_{y t} \angle \theta_{\mathrm{yt}}$ \\
\hline 3) Convert Ytotal to Ztotal using the following equation: $Y=1 / Z$ \\
\hline * ELI the ICE man is only used for series circuits, but if $\theta_{\mathrm{zt}}>0$ then Vin LEADS the source current \\
\hline 4) Calculate the source current using $I_{S}=$ Vin/Ztotal or skip step 3) and use $I_{S}=$ Vin $\cdot$ Ytotal \\
\hline $\begin{array}{l}\text { 5) Find currents through each component using Ohm's Law: } I_{R}=V i n / R, I_{L}=\operatorname{Vin} \cdot Y_{L}, I_{C}=\operatorname{Vin} \cdot Y_{C} \\
\text { * Note: From KVL you know that Vin }=V_{R}=V_{L}=V_{C}\end{array}$ \\
\hline 6) Write the currents and voltages in sinusoidal form: $A \cdot \sin (\omega \cdot t+\theta)$ \\
\hline
\end{tabular}

Before doing an example of solving an AC parallel RLC circuit the following figure shows a comparison from series and parallel resistor circuits. Notice how the current is the same through all components in the series circuit and the voltage is the same across all components in the parallel circuit.
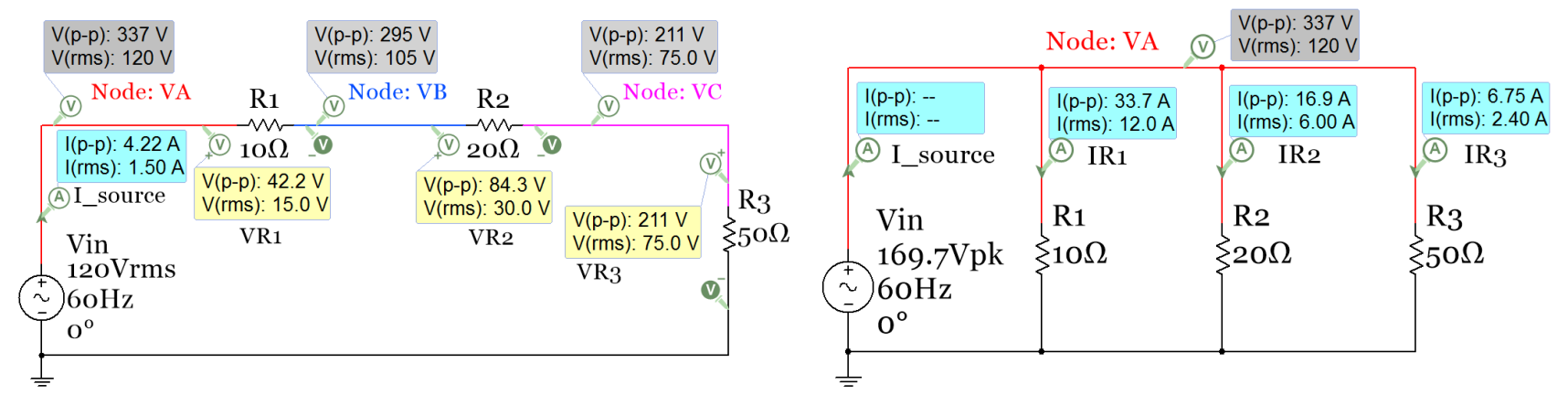

Figure 3.12: Series (Left) and Parallel (Right) circuit comparison with AC input voltage and only resistors. 
Figure 3.12 shows two different types of Multisim AC sources. The series circuit has a Multisim source called "AC_Power" and the voltage is in RMS. The parallel circuit has the Multisim source called "AC_Voltage" and the voltage is in peak voltage. The phasor voltage for the parallel circuit is $V_{\text {in }}=169.7 \angle 0^{\circ}$. Since $V i n$ is in peak voltage it is in the form required for phasor form. Since the units are Volts no units are listed after the phasor (Volts assumed if not units). The source in the series circuit is in RMS so it must be converted to peak before writing in phasor form: $V_{\text {in_pk }}=120 \mathrm{~V}_{\text {in_rms }} \cdot \sqrt{2}=169.7 \mathrm{~V} \rightarrow \mathrm{V}_{\text {in }}=169.7 \angle 0^{\circ}$. However, it is acceptable nomenclature to write the phasor amplitude in RMS if the units are included (i.e. $V_{\text {in }}=120 \angle 0^{\circ}$ Vrms).

Example 3.7) Solve for the following parallel RLC circuit and verify values in Multisim.

\begin{tabular}{|c|c|c|c|c|}
\hline & Node: VA & $\begin{array}{l}V(p-p): 23.9 \mathrm{~V} \\
\mathrm{~V} \\
\mathrm{~V}(\mathrm{rms}): 8.49 \mathrm{~V}\end{array}$ & & \multirow{6}{*}{$\begin{array}{l}\underline{\text { Steps } 1 \text { to } 3 \text { of Table } 3.3} \\
Y_{R}=G=1 / R=1 / 2=0.5 \Omega \\
B_{C}=C \cdot \omega=250 e-6 \cdot 600 \cdot \pi=0.471239 \Omega \\
Y_{C}=j B_{C}=j 0.471239 \Omega=\underline{\mathbf{0 . 4 7 1 2 3 9 \angle 9 0 ^ { 0 }}} \\
B_{L}=1 / L \cdot \omega=1 /(0.004 \cdot 600 \cdot \pi)=0.132629 \Omega \\
Y_{L}=-j B_{L}=-j 0.132629 \Omega=\underline{\mathbf{0 . 1 3 2 6 2 9} \angle-90^{0}} \\
Y_{\text {Tot }}=Y_{R}+Y_{C}+Y_{L}=0.5+j 0.33861 \Omega \\
Y_{\text {Tot }}=0.603868 \angle 34.10663^{0} \\
Z_{\text {Tot }}=1 / Y_{\text {Tot }}=\left(1 \angle 0^{0}\right) /\left(.603868 \angle 34.10663^{0}\right) \\
Z_{\text {Tot }}=\underline{\mathbf{1 . 6 5 5 9 9 1} \angle-34.1066^{0}}\end{array}$} \\
\hline $\begin{array}{l}I(p-p): 14.5 \mathrm{~A} \\
\text { I(rms): } 5.40 \mathrm{~A} \\
\text { (A) I source }\end{array}$ & $\begin{array}{l}I(p-p): 11.4 A \\
I(r m s): 4.05 A\end{array}$ & $\begin{array}{l}I(p-p): 11.9 A \\
I(r m s): 4.24 A A\end{array}$ & \multirow{2}{*}{$\begin{array}{l}I(p-p): 3.12 \mathrm{~A} \\
\text { I(rms): } 1.93 \mathrm{~A} \\
\text { (A) IR3 }\end{array}$} & \\
\hline (A) I_source & (A) IR1 & (A) IR2 & & \\
\hline Vin & $\mathrm{C}$ & $\mathrm{R}$ & & \\
\hline $\begin{array}{l}12 \mathrm{Vpk} \\
300 \mathrm{~Hz} \\
0^{\circ}\end{array}$ & $\stackrel{\perp}{\top} 250 \mu \mathrm{F}$ & $2 \Omega$ & $4 \mathrm{mH}$ & \\
\hline Figure & 13: Circuit & Example 3.7 & & \\
\hline
\end{tabular}

Steps 4 to 6 of Table 3.3 (Multisim Peak Values listed in Red Font and RMS values listed in Blue Font)

- Since the total impedance has an angle less than $0^{\circ}$ the source current, $\mathrm{I}_{\mathrm{s}}$, will lead the input voltage.

- $\operatorname{Vin}=\mathrm{V}_{\mathrm{R}}=\mathrm{V}_{\mathrm{L}}=\mathrm{V}_{\mathrm{C}}=12 \angle 0^{0}$ (Phasor Form) $\mathbf{V i n}(\mathbf{t})=\mathbf{V}_{\mathrm{R}}(\mathbf{t})=\mathbf{V}_{\mathrm{L}}(\mathbf{t})=\mathbf{v}_{\mathrm{C}}(\mathbf{t})=\mathbf{1 2} \cdot \sin \left(\mathbf{6 0 0} \cdot \boldsymbol{\pi}+\mathbf{0}^{\circ}\right.$ ) (Sinusoidal Form) I_source $=I_{S}=\operatorname{Vin} / Z_{\text {Total }}=\left(12 \angle 0^{0}\right) /\left(1.655991 \angle-34.1066^{0}\right)=\mathbf{7 . 2 4 6 4 \angle 3 4 . 1 0 6 6 ^ { 0 }}$ Multisim: $I_{s}=14.5 / 2=7.25 A$

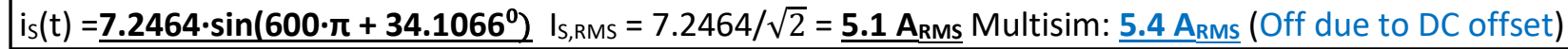

- $\quad I_{R}=\operatorname{Vin} / R=\left(12 \angle 0^{0}\right) /\left(2 \angle 0^{0}\right)=\underline{6} \angle 0^{0}$ Multisim: $I_{R}=11.9 A_{p-p} / 2=\underline{5.95 A}$ (Close to calculated)

- $\mathrm{I}_{\mathrm{R}}(\mathrm{t})=\underline{6 \cdot \sin \left(600 \cdot \pi+0^{0}\right)} \mathrm{I}_{\mathrm{R}, \mathrm{RMS}}=6 / \sqrt{2}=\underline{4.24} \mathrm{~A}_{\mathrm{RMS}}$ Multisim: $4.24 \mathrm{~A}_{\mathrm{RMS}}$ (Correct since no DC offset is added) $I_{L}=\operatorname{Vin} \cdot Y_{L}=\left(12 \angle 0^{0}\right) \cdot\left(0.132629 \angle-90^{0}\right)=\underline{1.59 \angle-90^{0}}$ Multisim: $I_{L}=3.12 A_{p-p} / 2=\underline{1.56 ~ A}$ (Close to calculated) $I_{L}(t)=\underline{1.59} \cdot \sin \left(600 \cdot \pi-90^{\circ}\right) I_{L, R M S}=1.59 / \sqrt{2}=\underline{1.13} A_{R M S}$ Multisim: $\underline{1.93} A_{R M S}$ (WAY Off due to DC offset)

- $\mathrm{I}_{C}=\operatorname{Vin} \cdot \mathrm{Y}_{C}=\left(12 \angle 0^{\circ}\right) \cdot\left(0.471239 \angle 90^{\circ}\right)=\underline{\mathbf{5 . 6 5} \angle 90^{\circ}}$ Multisim: $\mathrm{I}_{\mathrm{R}}=11.4 \mathrm{Ap-p} / 2=\underline{5.7 \mathrm{~A}}$ (Close to calculated)

- $I_{C}(t)=\underline{5.65} \cdot \sin \left(600 \cdot \pi+90^{\circ}\right) I_{C, R M S}=5.65 / \sqrt{2}=\underline{4} A_{R M S}$ Multisim: $4.05 A_{R M S}$ (Close: $n o$ DC offset is added)

The reason the RMS $I_{S}$ and $I_{L}$ don't match the calculations is due to the DC offset that is wrongly included in the simulation as shown in Figure 3.14 (this link gives more info). Here are some options to avoid this error.

1) Measure only the peak to peak probe current in Multisim and convert it to RMS $\rightarrow I_{R M S}=I_{p-p} /(2 \sqrt{2})$

2) Calculate $I_{s}$ and use a current source instead of a voltage source. There is no DC offset in Figure 3.14.

3) Double click on the inductor and enter a DC initial condition value to negate what Multisim adds in.

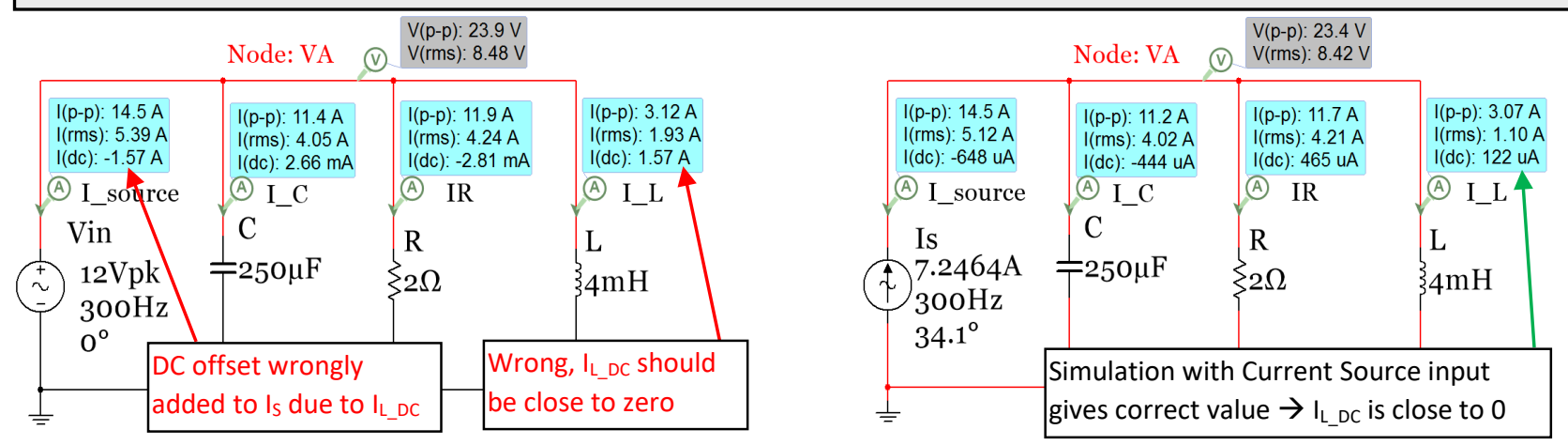

Figure 3.14: Parallel circuit in Example 3.7 with AC Voltage source (Left) and AC Current Source (Right). 
All of the voltages and currents have been calculated above and the magnitudes were verified in Multisim. To verify the phase the oscilloscope is needed. To remove the DC offset of the inductor, method 3 described in Figure 3.14 will be used. Figure 3.15 shows the dialog that comes up when you double click on the inductor. Since the DC offset was measured to be 1.57A, a value of $-1.57 \mathrm{~A}$ is entered into the initial condition box to correct for the DC offset. Figure 3.16 (Left) shows the plot of Vin and $I_{s}$ for the initial circuit without modifying the initial condition of the inductor. Due the DC offset the peak value of $I_{s}$ is measured to be $8.738 \mathrm{~A}$ which is about 1.5 A higher than the calculated value of $I_{\text {s_pk }}(7.2464 \mathrm{~A})$. In the right plot of Figure 3.16 the initial condition of the inductor is included and the DC offset is removed from both $\mathrm{I}_{\mathrm{L}}$ and $\mathrm{I}_{\mathrm{S}}$.

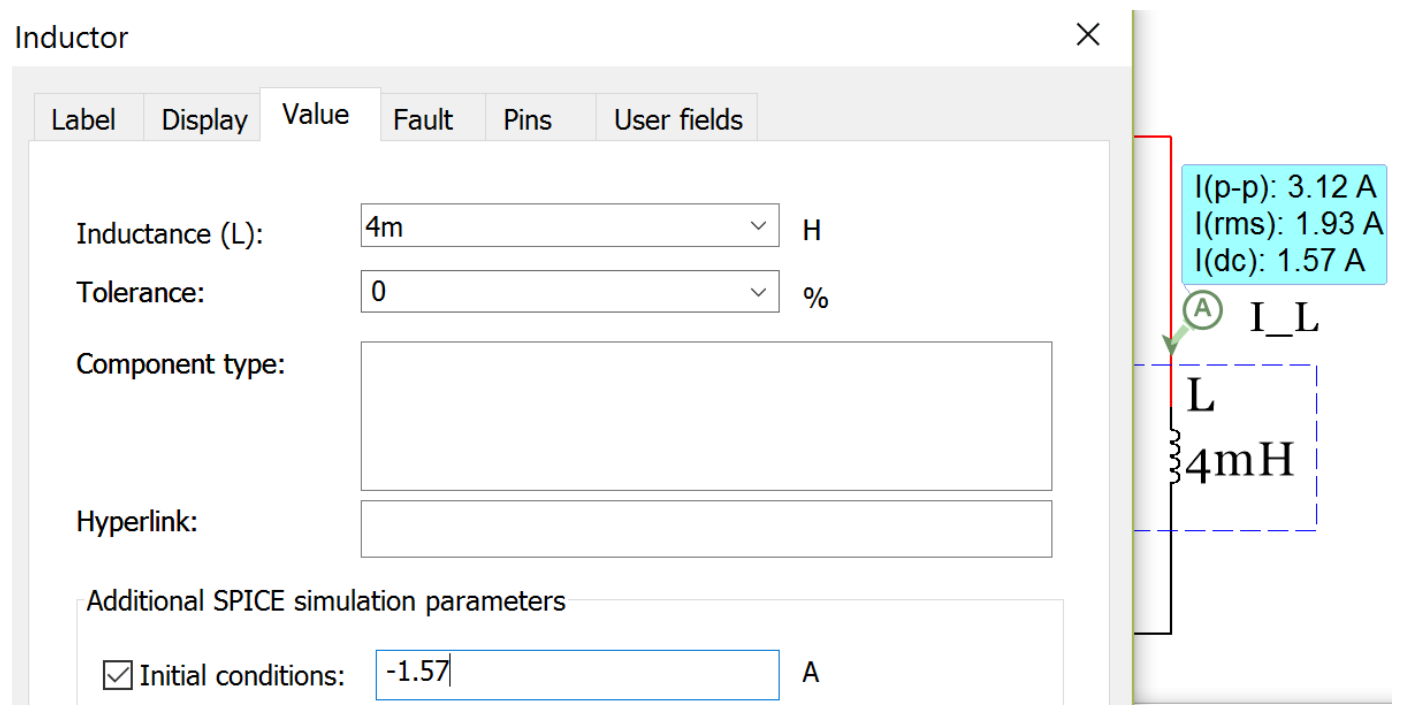

Figure 3.15: Inductor dialogue box to enter the DC offset as the initial condition.

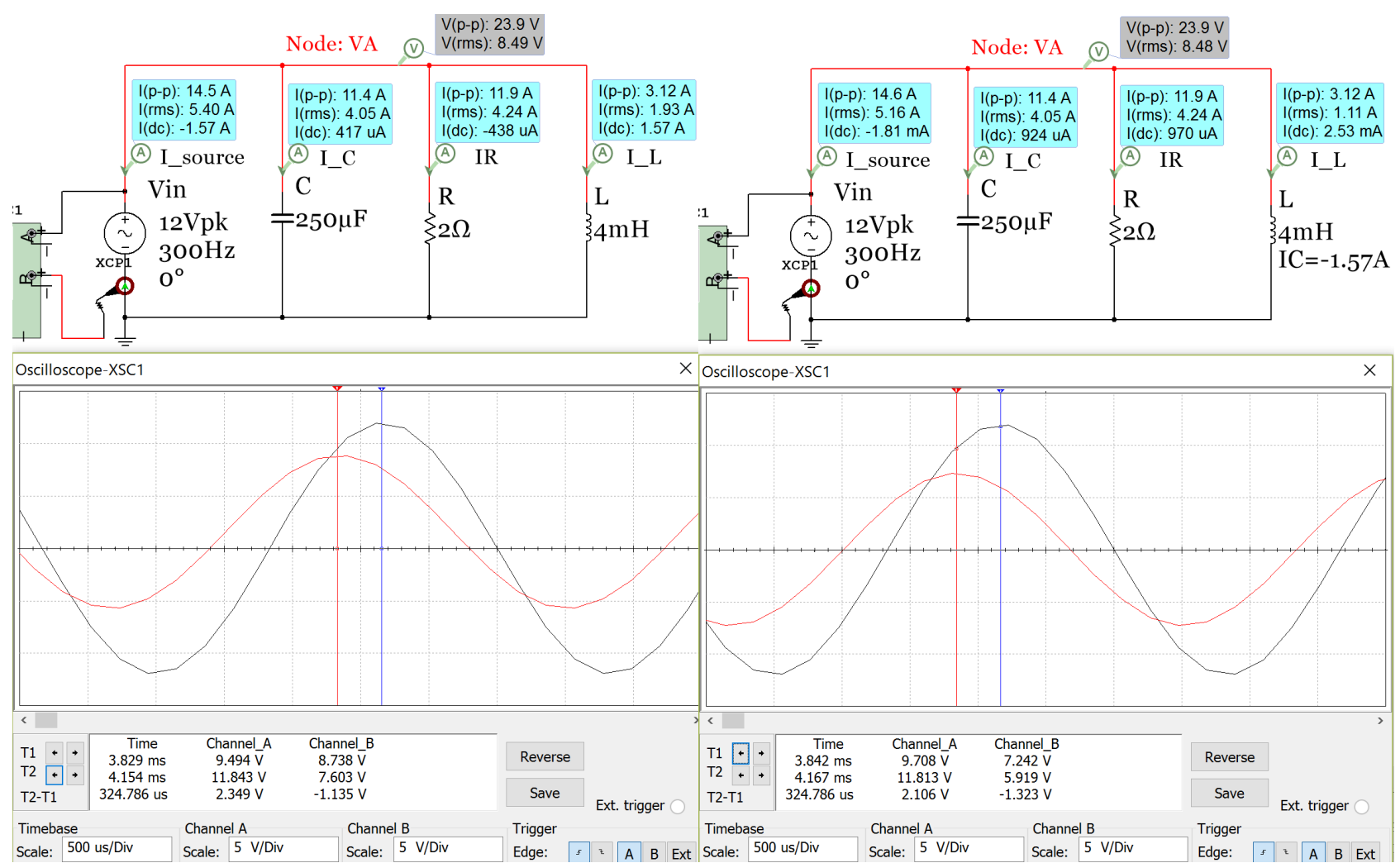

Figure 3.16: Parallel RLC circuit from Example 3.7 - Phase Verification in Multisim. 
The time shift, $t_{s}$, in Figure 3.16 is measured to be $-0.324786 \mathrm{~ms}$ ( $t_{s}$ is negative because its sinusoidal cycle starts before $\mathrm{Vin})$. The phase of $\mathrm{I}_{\mathrm{s}}$ is calculated below and matches the calculated value $\left(\underline{\mathbf{3 4 . 1 ^ { \circ }}}\right)$ fairly closely.

- $\theta=-t_{s} \cdot \omega=-(-0.000324786 \mathrm{sec}) \cdot(300 \cdot 2 \pi$ radians $/ \mathrm{sec})=0.6122$ radians $\rightarrow 3^{\circ}$

- Even though the ELI the ICE man pneumonic doesn't apply to parallel circuits it DOES apply once the total impedance is determined and you want to compare the source current to the input voltage. In this example Is LEADS Vin (ICE) because the total impedance phase angle is in quadrant IV $\left(-34.1^{\circ}\right)$. This is verified in Figure 3.16.

Figure 3.17 shows the capacitor current and Vin (Left) and the inductor current and Vin (Right). Recall that the phase shift is positive if the time shift is negative (or shifted left) and vice versa.

- Both plots below show the time difference between peaks is 0.8333 ms. Multiplying the magnitude of the time shift by the angular frequency of Vin $(600 \pi)$ results in $\pi / 2\left(\right.$ or $\left.90^{\circ}\right)$.

- It can be clearly seen that $I_{c}$ has a $+90^{\circ}$ phase shift since it is shifted $1 / 4$ period to the left of Vin.

- The peak current of $I_{C}$ is measured to be $\underline{5.649 \mathrm{~A}}$. This is very close to the calculated value of $5.65 \mathrm{~A}$.

- It can be clearly seen that $I_{L}$ has a $-90^{\circ}$ phase shift since it is shifted $1 / 4$ period to the right of Vin.

- The peak current of $I_{L}$ is measured to be $1.549 \mathrm{~A}$. This is very close to the calculated value of $1.59 \mathrm{~A}$.

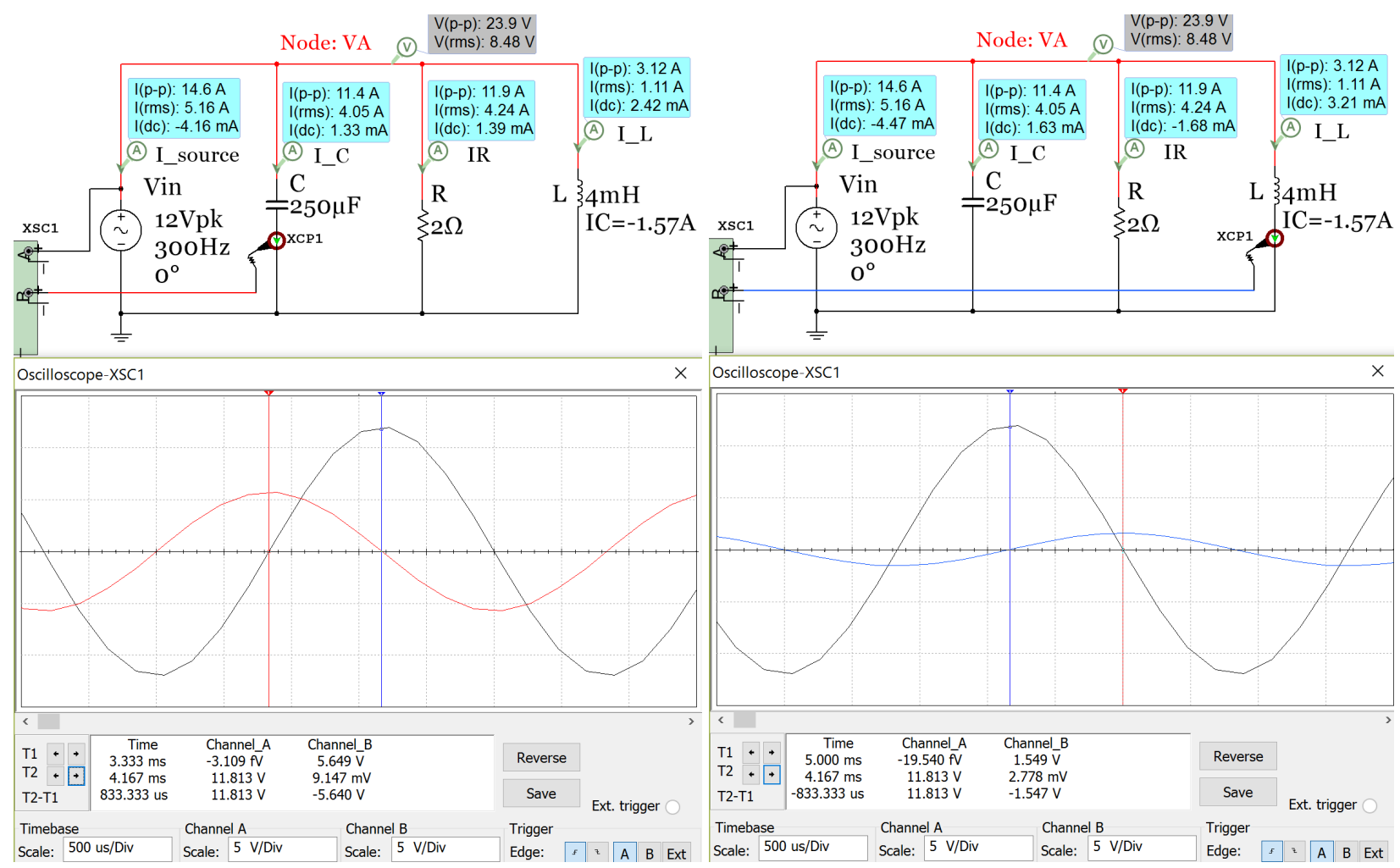

Figure 3.17: Example 3.7 verification: Left: $I_{C}(R e d)$ and Vin (Black) plots, Right: $I_{L}$ (Blue) and Vin (Black) plots

\section{Section 3.4 - AC Power Calculations}

In section 1.3.3 the RMS voltage was found to be an important quantity in AC circuits because it allows the AC power delivered to a load to be equated to the DC power. Vrms can be defined as the magnitude of AC voltage that produces the same amount of average power on a load as an equivalent DC voltage, as shown in Figure 1.13. As previously discussed, if an AC circuit is frozen at a specific time the current and voltage at that time is simply a DC value that is referred to as "instantaneous". Example 3.8 below shows the series circuit used in Figure 3.12 with power probes included. The instantaneous voltage $\left(V_{\text {inst }}\right)$ and current $\left(l_{\text {inst }}\right)$ can be multiplied 
together to get the instantaneous power ( $\left.P_{\text {inst }}\right)$ as shown in Equation 3.1. Since $P_{\text {inst }}$ constantly changes and only applies to a specific instant of time it is not frequently used for AC power. Instead, the average power (shown in Equation 3.2) is the primary type of power used for circuits with AC sources. The average power is can be determined by Equation 3.2 or it can be determined by finding the average instantaneous power over 1 period (see Table 3.4 and Figure 3.20 for an example).

[3.1] $P_{\text {inst }}=I_{\text {inst }} \cdot V_{\text {inst }}$ (This is the same as freezing the circuit and treating values as DC)

[3.2] $P_{\text {avg }}=P=I_{R M S} \cdot V_{R M S} \cdot P F$ ( $\mathbf{P}$ or $P_{\text {avg }}$ is called $\underline{\text { Real Power. }}$ The units are $\underline{\text { Watts }}$ )

[3.3] $P F=$ Power Factor $=\cos \theta_{Z t}$ ( $\left(\theta_{\mathrm{zt}}\right.$ is the total impedance phase angle)

Note: If $\theta_{\mathrm{Zt}}>0$ (ELI) the power factor (PF) is said to be "Lagging." If $\theta_{\mathrm{Zt}}<0$ (ICE) the PF is "Leading."

[3.4] $S=I_{R M S} \cdot V_{R M S}$ (S is called Apparent Power. The units are $\underline{\mathbf{V} \cdot \mathbf{A}}$ )

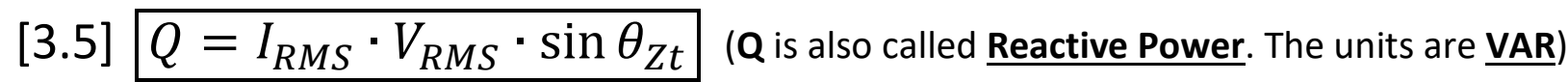

$\mathrm{P}, \mathrm{S}$, and $\mathrm{Q}$ make up what is referred to as the Power Triangle. This "triangle" (or vector in phasor form) has the same angle as the total impedance, but the amplitude is equal to $S$ (or $V_{\text {RMS }} \cdot I_{\text {RMS }}$ ).

Resistors only have real power (P). Capacitors and inductors only have reactive power (ㅇ). The apparent power $(\mathrm{S})$ is thought of as the total power that is possible for the ideal case where $\mathrm{Q}=0$.

Example 3.8) Calculate the instantaneous and average power magnitudes for each component and source in the circuit below and show that the magnitude of the power supplied is equal to the power dissipated.

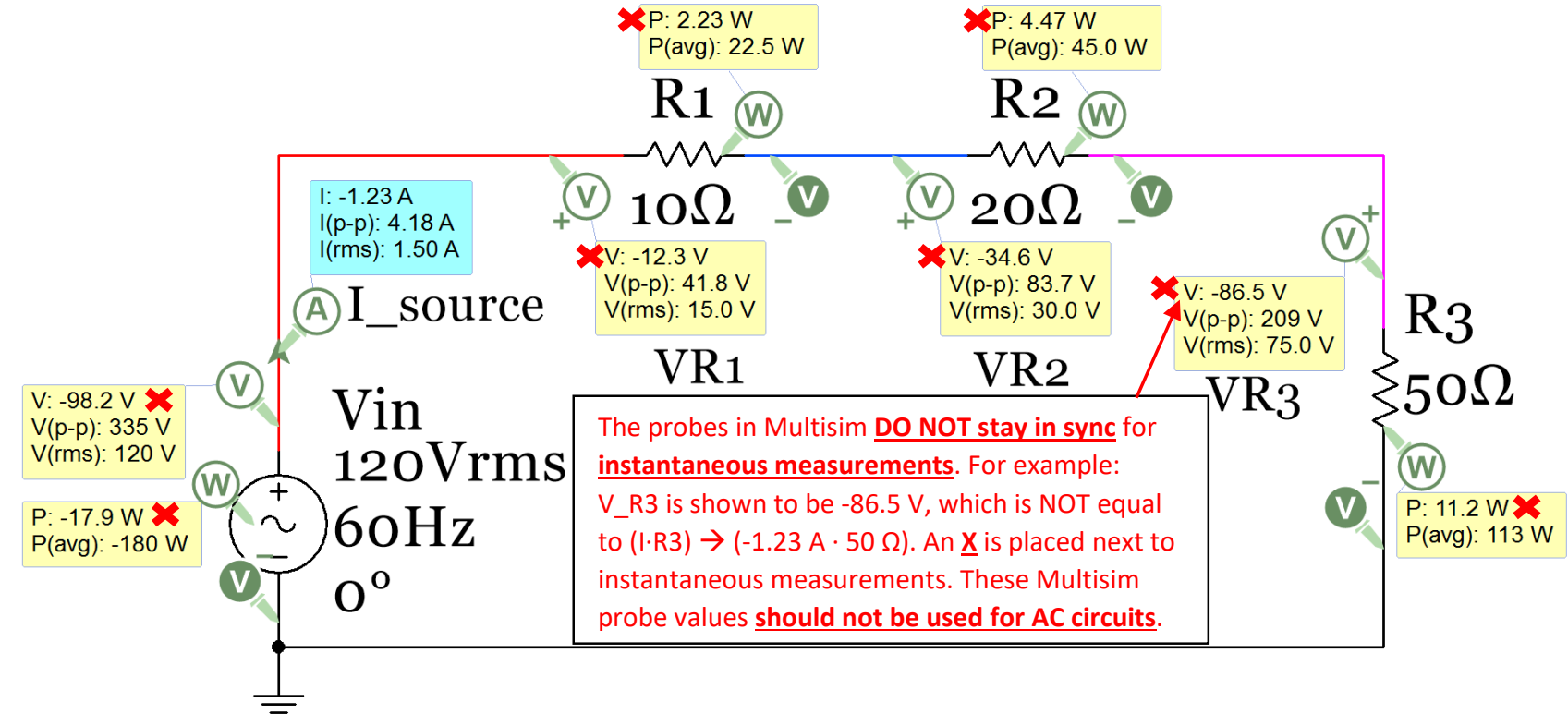

Figure 3.18: Circuit for Example 3.8

Since there are only resistors in this circuit $\theta_{Z t}=0$ and the power factor $=1$ so $Q=0$ and $S=P=V_{R M S} \cdot I_{R M S}$. The Multisim power probes show instantaneous power $(\mathrm{P})$ and average power ( $\mathrm{P}(\operatorname{avg}))$. The magnitude of the 
instantaneous power supplied will constantly change between 0 and $V_{\text {in_pk }} \cdot l_{\text {in_pk, }}$, but the average (or real) power supplied will remain constant at a value of $V_{R M S} \cdot I_{R M S}$. The magnitude of the supply power is equal to the dissipated power for both $\mathrm{P}_{\text {inst }}$ and $\mathrm{P}_{\text {avg }}$ as shown below. Recall, the sign of supplied power is always set as a negative. For instantaneous power there are infinite solutions depending on when the circuit is paused (or frozen in time). Since the instantaneous measurements in Multisim aren't in sync (i.e. not associated with the same moment in time) they will not be displayed in future circuits. They were only displayed in this example to point out this limitation in Multisim and to make sure you don't use them in any calculations.

Instantaneous power calculations: (This is the same process used in DC circuits): At the moment the Multisim simulation is paused the voltage source has a voltage of $\mathbf{- 9 8 . 2 \mathrm { V }}$. The following calculations show that the magnitude of the power supplied is equal to the power dissipated at this moment in time. The calculated values won't match the Multisim values because the instantaneous probe values are not in sync.

- $\mathrm{R}_{\text {Total }}=10 \Omega+20 \Omega+50 \Omega=\underline{80 \Omega}$, Current: $I_{\text {inst }}=V_{\text {inst }} / R_{\text {Total }}=-98.2 \mathrm{~V} / 80 \Omega=1.2275 \mathrm{~A}$

- $\mathrm{P}_{\text {supplied }}=\mathrm{V}_{\text {inst }} \cdot \mathrm{I}_{\text {inst }}=(-98.2 \mathrm{~V}) \cdot(1.2275 \mathrm{~A})=\underline{\mathbf{1 2 0 . 5} \mathbf{W}}$ (power is always listed as positive in this eBook)

- $P_{10 \Omega}=(1.2275 A)^{2} \cdot(10 \Omega)=\underline{15.07 W}, P_{20 \Omega}=(1.2275 A)^{2} \cdot(20 \Omega)=\underline{30.14 W}, P_{50 \Omega}=(1.2275 A)^{2} \cdot(50 \Omega)=\underline{75.33 \mathrm{~W}}$

- $\sum P_{\text {dissipate }}=P_{10 \Omega}+P_{20 \Omega}+P_{50 \Omega}=15.07 \mathrm{~W}+30.14 \mathrm{~W}+75.33 \mathrm{~W}=\underline{\mathbf{1 2 0 . 5} \mathbf{W}}=\sum \mathrm{P}_{\text {supplied }}$

Average power calculations: This is the same process as before, but the current and voltage instantaneous values are replaced with RMS values. Each value is calculated below and the Multisim measurement is included in blue font and shown next to the calculated value to be used as verification. For the voltage across the resistors $\mathbf{I}^{\mathbf{2}} \cdot \mathbf{R}$ was used previously and $\mathbf{I} \cdot \mathbf{V}$ is used below. Another option that can be used is $\mathbf{V}^{\mathbf{2}} / \mathbf{R}$.

- $\mathrm{R}_{\text {Total }}=10 \Omega+20 \Omega+50 \Omega=\underline{80 \Omega}$, Current: $I_{\text {RMS }}=V_{\text {in-RMS }} / R_{\text {Total }}=120 \mathrm{~V} / 80 \Omega=\underline{1.5 A}$ Multisim $\rightarrow(\underline{1.5 A})$

- $P_{\text {supplied }}=V_{\text {in_RMs }} I_{\text {RMS }}=(120 \mathrm{~V}) \cdot(1.5 \mathrm{~A})=\underline{\mathbf{1 8 0 ~ W}}(\underline{180 \mathrm{~W}}) \rightarrow$ Always use the $\underline{\mathbf{P}=\mathrm{V} \cdot \mathrm{I}}$ equation for sources.

- $\mathrm{V}_{10 \Omega}=(1.5 \mathrm{~A}) \cdot(10 \Omega)=\underline{15 \mathrm{~V}}(\underline{\underline{15 V}}), \mathrm{V}_{20 \Omega}=(1.5 \mathrm{~A}) \cdot(20 \Omega)=\underline{30 \mathrm{~V}}(\underline{30 \mathrm{~V}}), \mathrm{V}_{50 \Omega}=(1.5 \mathrm{~A}) \cdot(50 \Omega)=\underline{75 \mathrm{~V}}(\underline{75 \mathrm{~V}})$

- $\mathrm{P}_{10 \Omega}=(1.5 \mathrm{~A}) \cdot(15 \mathrm{~V})=\underline{22.5 \mathrm{~W}}(\underline{22.5 \mathrm{~W}}), \mathrm{P}_{20 \Omega}=(1.5 \mathrm{~A}) \cdot(30 \mathrm{~V})=\underline{45 \mathrm{~W}}(\underline{45 \mathrm{~W}}), \mathrm{P}_{50 \Omega}=(1.5 \mathrm{~A}) \cdot(75 \mathrm{~V})=\underline{112.5 \mathrm{~W}}(\underline{113 \mathrm{~W}})$

- $\sum \mathrm{P}_{\text {dissipate }}=\mathrm{P}_{10 \Omega}+\mathrm{P}_{20 \Omega} \mathrm{P}_{50 \Omega}=22.5 \mathrm{~W}+45 \mathrm{~W}+112.5 \mathrm{~W}=\underline{180 \mathrm{~W}}=\sum \mathrm{P}_{\text {supplied }}$

Example 3.8 showed that the average power in AC circuits uses the RMS voltage and currents in the power formula of Equation 3.2. For AC circuits with only resistors the current and voltages are always "in phase" with each other which maximizes the average power. For RLC circuits the capacitors and/or inductors in the circuit causes the current and voltage to be out of phase (as explained previously with the ELI the ICE man pneumonic), which results in a reduced average (or real) power. Figure 3.19 shows an AC circuit with only a resistor and a circuit with a capacitor added that results in the voltage and current being out of phase. In Table 3.4 and Figure $\underline{3.20}$ provide a detailed analysis showing the effects of adding a capacitor (resulting in the current and voltage going out of phase) on the instantaneous and average power.

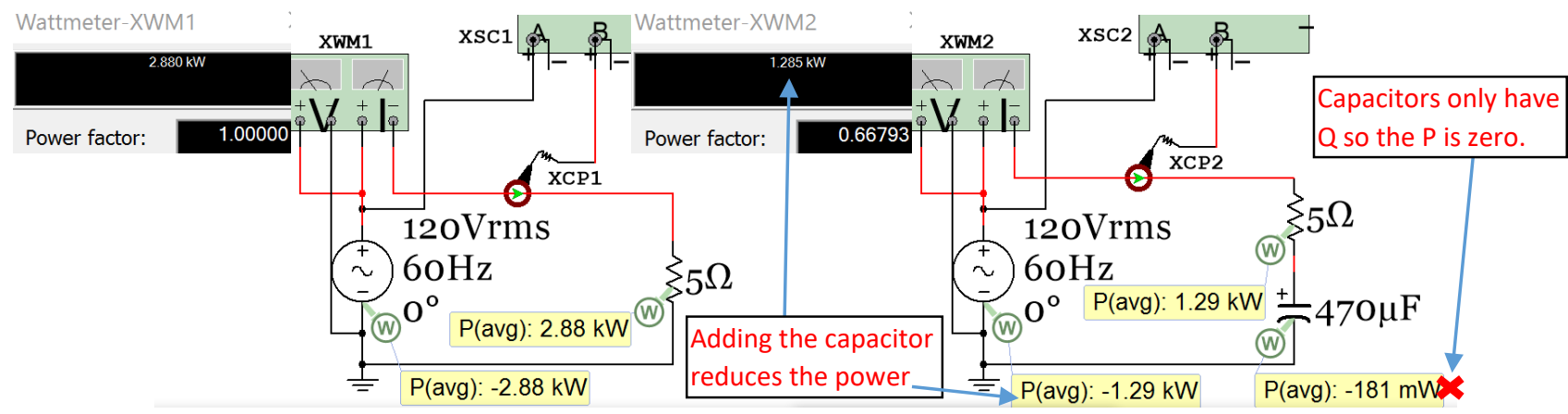

Figure 3.19: Circuit showing that adding a capacitor (or inductor) reduces the average power. 
Table 3.4) Instantaneous Values for 2 RC circuits. Case 1: (No Capacitor), Case 2: $470 \mu \mathrm{F}$ Capacitor added.

\begin{tabular}{|c|c|c|c|c|c|}
\hline \multicolumn{2}{|c|}{1 Period with 40 pts } & \multicolumn{2}{|c|}{ Case 1: No Cap. } & \multicolumn{2}{|c|}{ Case $2,470 \mu \mathrm{F}$} \\
\hline $\mathrm{t}(\mathrm{ms})$ & V_inst & I_inst & P_inst & I_inst & $P$ _inst \\
\hline 0 & 0 & 0 & 0 & 0.557 & 0 \\
\hline 0.25 & 0.939 & 0.188 & 0.176 & 0.679 & 0.637 \\
\hline 0.5 & 1.854 & 0.371 & 0.688 & 0.784 & 1.454 \\
\hline 0.75 & 2.724 & 0.545 & 1.484 & 0.87 & 2.37 \\
\hline 1 & 3.527 & 0.705 & 2.488 & 0.934 & 3.295 \\
\hline 1.25 & 4.243 & 0.849 & 3.6 & 0.976 & 4.139 \\
\hline 1.5 & 4.854 & 0.971 & 4.712 & 0.993 & 4.82 \\
\hline 1.75 & 5.346 & 1.069 & 5.716 & 0.986 & 5.271 \\
\hline 2 & 5.706 & 1.141 & 6.512 & 0.955 & 5.447 \\
\hline 2.25 & 5.926 & 1.185 & 7.024 & 0.9 & 5.332 \\
\hline 2.5 & 6 & 2 & 7.2 & 0.823 & 4.936 \\
\hline 2.75 & 5.926 & 1.185 & 7.024 & 0.725 & 4.299 \\
\hline 3 & 5.706 & 1.141 & 6.512 & 0.61 & 3.482 \\
\hline 3.25 & 5.346 & 1.069 & 5.716 & 0.48 & 2.566 \\
\hline 3.5 & 4.854 & 0.971 & 4.712 & 0.338 & 1.641 \\
\hline 3.75 & 4.243 & 0.849 & 3.6 & 0.188 & 0.797 \\
\hline 4 & 3.527 & 0.705 & 2.488 & 0.033 & 0.116 \\
\hline 4.25 & 2.724 & 0.545 & 1.484 & -0.123 & -0.335 \\
\hline 4.5 & 1.854 & 0.371 & 0.688 & -0.276 & -0.511 \\
\hline 4.75 & 0.939 & 0.188 & 0.176 & -0.422 & -0.396 \\
\hline 5 & 0 & 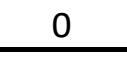 & 0 & -0.557 & 0 \\
\hline 5.25 & -0.939 & -0.188 & 0.176 & -0.679 & 0.637 \\
\hline 5.5 & -1.854 & -0.371 & 0.688 & -0.784 & 1.454 \\
\hline 5.75 & -2.724 & -0.545 & 1.484 & -0.87 & 2.37 \\
\hline 6 & -3.527 & -0.705 & 2.488 & -0.934 & 3.295 \\
\hline 6.25 & -4.243 & -0.849 & 3.6 & -0.976 & 4.139 \\
\hline 6.5 & -4.854 & -0.971 & 4.712 & -0.993 & 4.82 \\
\hline 6.75 & -5.346 & -1.069 & 5.716 & -0.986 & 5.271 \\
\hline 7 & -5.706 & -1.141 & 6.512 & -0.955 & 5.447 \\
\hline 7.25 & -5.926 & -1.185 & 7.024 & -0.9 & 5.332 \\
\hline 7.5 & -6 & -1.2 & 7.2 & -0.823 & 4.936 \\
\hline 7.75 & -5.926 & -1.185 & 7.024 & -0.725 & 4.299 \\
\hline 8 & -5.706 & -1.141 & 6.512 & -0.61 & 3.482 \\
\hline 8.25 & -5.346 & -1.069 & 5.716 & -0.48 & 2.566 \\
\hline 8.5 & -4.854 & -0.971 & 4.712 & -0.338 & 1.641 \\
\hline 8.75 & -4.243 & -0.849 & 3.6 & -0.188 & 0.797 \\
\hline 9 & -3.527 & -0.705 & 2.488 & -0.033 & 0.116 \\
\hline 9.25 & -2.724 & -0.545 & 1.484 & 0.123 & -0.335 \\
\hline 9.5 & -1.854 & -0.371 & 0.688 & 0.276 & -0.511 \\
\hline 9.75 & -0.939 & -0.188 & 0.176 & 0.422 & -0.396 \\
\hline 10 & 0 & 0 & 0 & 0.557 & 0 \\
\hline
\end{tabular}

Table 3.4 shows 40 instantaneous data points over 1 period of $\mathrm{V}, \mathrm{I}$, and $\mathrm{P}$ of the circuits shown in Figure 3.19. The instantaneous values are plotted over 1 period for both cases. To determine the average (or real) power (Pavg) take an average of the instantaneous power values over 1 period or use Equation 3.2. $\rightarrow P_{\text {avg }}=V_{\mathrm{RMS}} \cdot I_{\mathrm{RMS}} \cdot \cos \left(\theta_{\mathrm{Zt}}\right)$
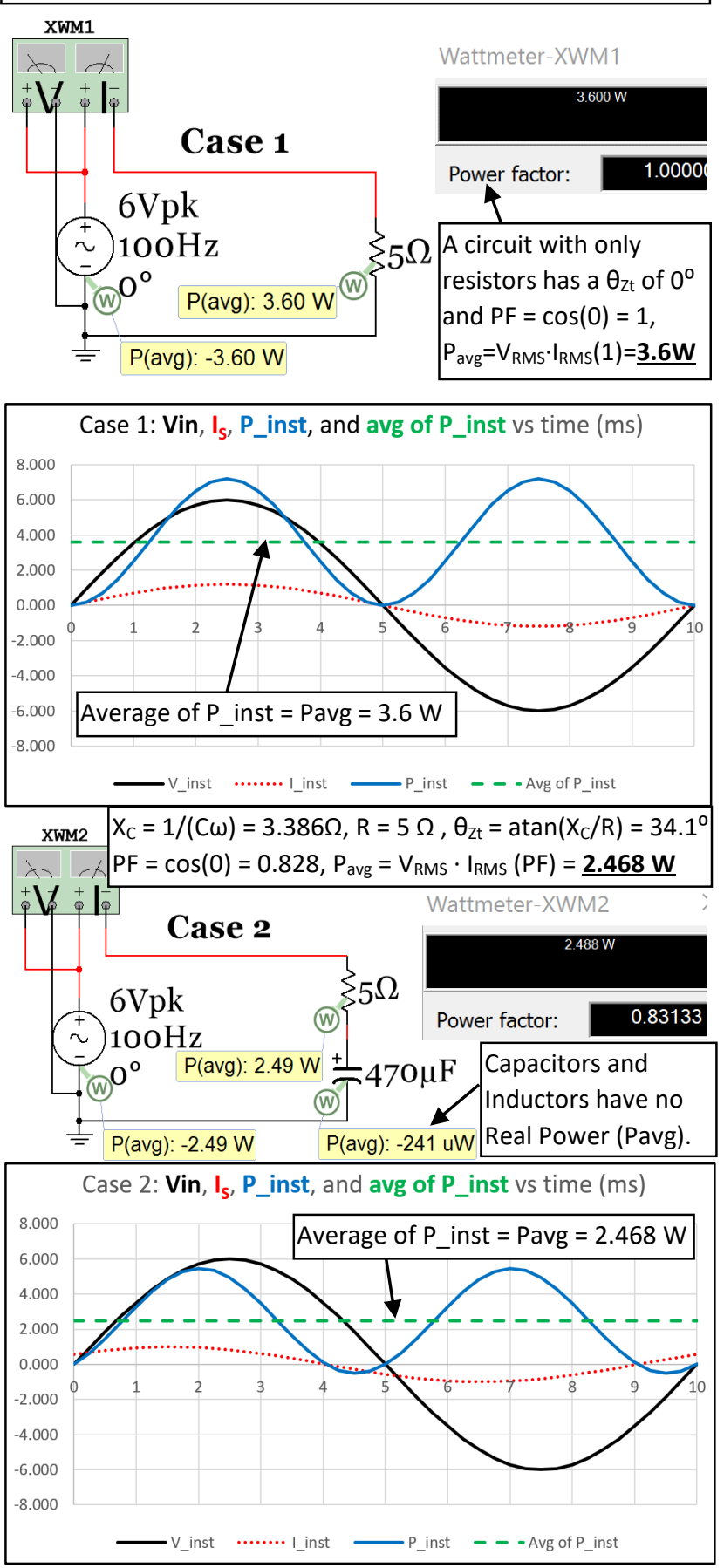

Figure 3.20: Data in Table 3.4 plotted 
Example 3.9) For the following RLC series circuit calculate the values of $v_{R 1}(t), v_{L 2}(t), S, P$, and $Q$ and draw the power triangle. Determine what component needs to be added to the circuit to eliminate the apparent power and produce a power factor of 1.

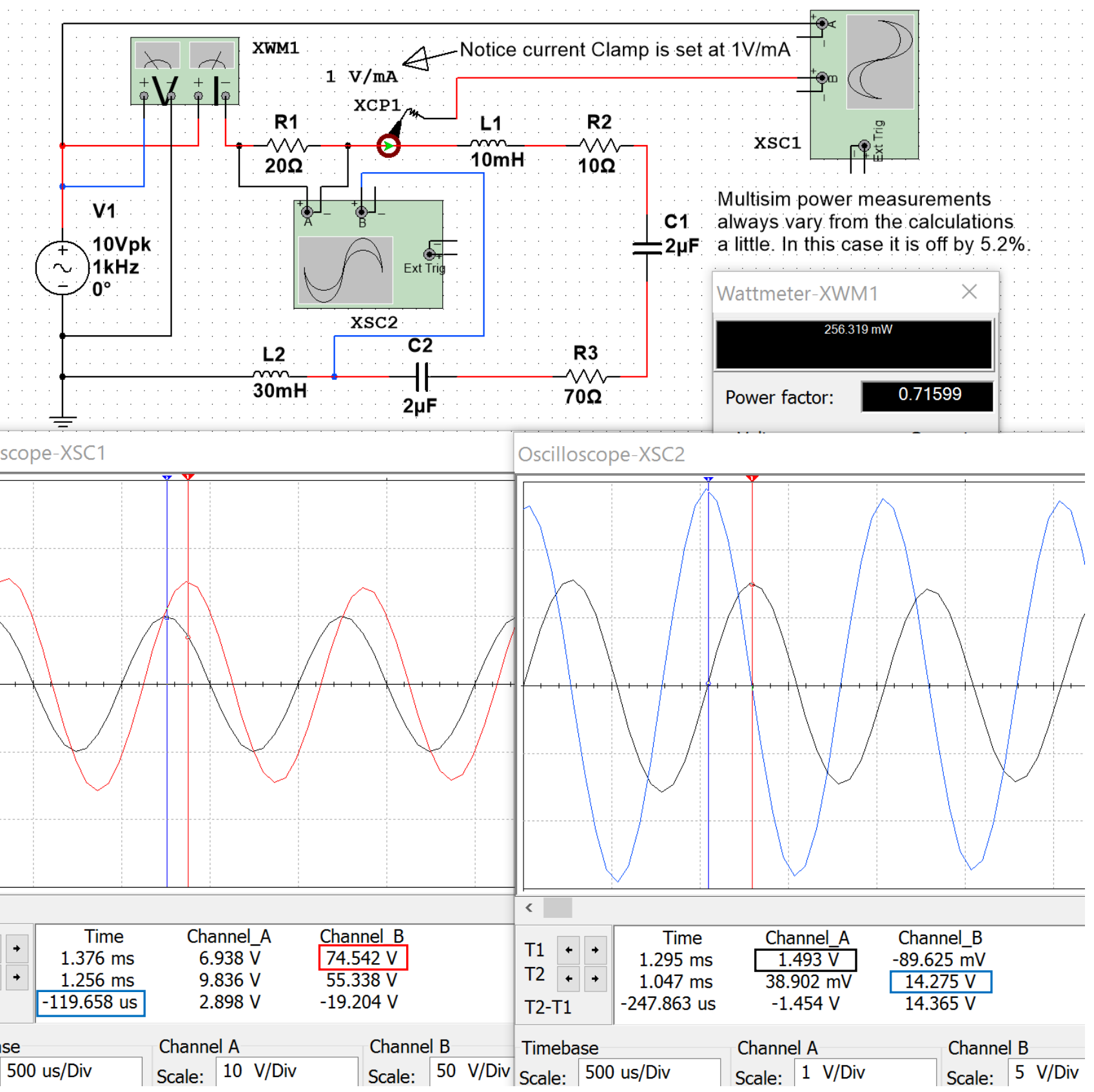

Figure 3.21: Circuit for Example 3.9

- $\omega=2 \pi \cdot f=2 \pi \cdot 1000=\underline{6283.2 \mathrm{rad} / \mathrm{sec}} \cdot \mathrm{X}_{\mathrm{L} 1}=\mathrm{L}_{1} \cdot \omega=\underline{62.8 \Omega}, \mathrm{X}_{\mathrm{L} 2}=\mathrm{L}_{2} \cdot \omega=\underline{188.5 \Omega}, \mathrm{X}_{\mathrm{C} 1}=\mathrm{X}_{\mathrm{C} 2}=1 /(2 \mu \mathrm{F} \cdot \omega)=\underline{79.6 \Omega}$

- $Z_{\text {Total }}=R 1+j X_{L 1}+R 2+R 3-j X_{C 1}+j X_{L 2}-j X_{C 2}=20+j \underline{62.8}+10+70-j \underline{79.6}+j \underline{188.5}-j \underline{79.6}$

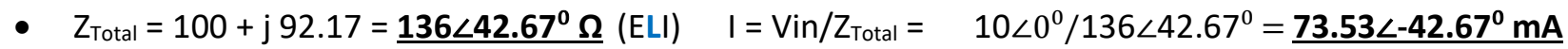

- Time shift, $\mathrm{t}_{\mathrm{s}}$, of current $=-\theta / \omega=-42.67^{\circ} \cdot\left(\pi / 180^{\circ}\right) / 6283.2 \mathrm{rad} / \mathrm{sec}=\underline{\mathbf{0 . 1 1 9} \mathbf{~ m s}}$ (0.1197 $\mathrm{ms}$ in Multisim)

- $\left.\quad V_{R 1}=I \cdot R 1=\left(73.53 \angle-42.67^{0} \mathrm{~mA}\right) \cdot\left(20 \angle 0^{0}\right)=1.47 \angle-42.67^{\circ}(\underline{1.493}) \rightarrow \underline{V}_{R 1}(\mathbf{t})=\underline{1.471 \cdot \sin (2000 \pi \cdot t-0.237 \pi}\right)$

- $\mathrm{V}_{\mathrm{L} 2}=\mathrm{I} \cdot \mathrm{R} 1=\left(73.53 \angle-42.67^{\circ} \mathrm{mA}\right) \cdot\left(188.5 \angle 90^{\circ}\right)=13.9 \angle 47.33^{\circ}(\underline{14.275}) \rightarrow \underline{\mathbf{v}_{\mathrm{L} 2}}(\mathbf{t})=\mathbf{1 3 . 9} \cdot \sin (\mathbf{2 0 0 0 \pi} \cdot \mathbf{t}+\mathbf{0 . 2 6 3 \pi})$

- $\quad$ Figure 3.21 shows $V_{R 1}$ and $V_{L 2}$ are $90^{\circ}$ out of phase. Calculations agree $\rightarrow \theta_{V L 2}-\theta_{V R 1}=47.33^{\circ}--42.67^{\circ}=90^{\circ}$ 
The power calculations are shown below. $\mathrm{PF}=\cos \left(\theta_{\mathrm{Zt}}\right)=\cos \left(42.67^{\circ}\right)=\underline{\mathbf{0 . 7 3 5 3}}(\underline{\mathbf{0 . 7 1 5 9 9}}$ in Multisim)

- $S=I_{R M S} \cdot V_{R M S}=(0.07353 / \sqrt{2}) \cdot(10 / \sqrt{2})=\underline{\mathbf{0 . 3 6 8} \mathbf{V} \cdot \mathbf{A}}$

- $Q=I_{\text {RMS }} \cdot V_{\text {RMS }} \cdot \sin \left(\theta_{\mathrm{Zt}}\right)=(0.07353 / \sqrt{2}) \cdot(10 / \sqrt{2}) \sin \left(42.67^{\circ}\right)=\underline{\mathbf{0 . 2 4 9} \text { VAR }}$

- $\mathrm{P}=\mathrm{I}_{\mathrm{RMS}} \cdot \mathrm{V}_{\mathrm{RMS}} \cdot \cos \left(\theta_{Z \mathrm{t}}\right)=(0.07353 / \sqrt{2}) \cdot(10 / \sqrt{2}) \cos \left(42.67^{\circ}\right)=\underline{\mathbf{0 . 2 7} \mathbf{W}}(\underline{\mathbf{0 . 2 5 6 3 1 9} \mathbf{W}}$ in Multisim)

To get the power factor equal to 1 a capacitor must be added to move $\mathrm{Zt}$ from quadrant I to on the $\mathrm{x}$ axis.

- $\mathrm{Z}_{\text {Total }}=100+\mathrm{j}$ 92.17, Need $\mathrm{X}_{\mathrm{C}}$ of added capacitor equal to $92.17 \Omega \rightarrow \mathrm{C}=1 /\left(\mathrm{X}_{\mathrm{C}} \cdot \omega\right)=\underline{\mathbf{1 . 7 2 6 7} \boldsymbol{\mu F}}$

- This capacitor is added into the circuit below. The current is now in phase with Vin and PF $=\sim 1$.

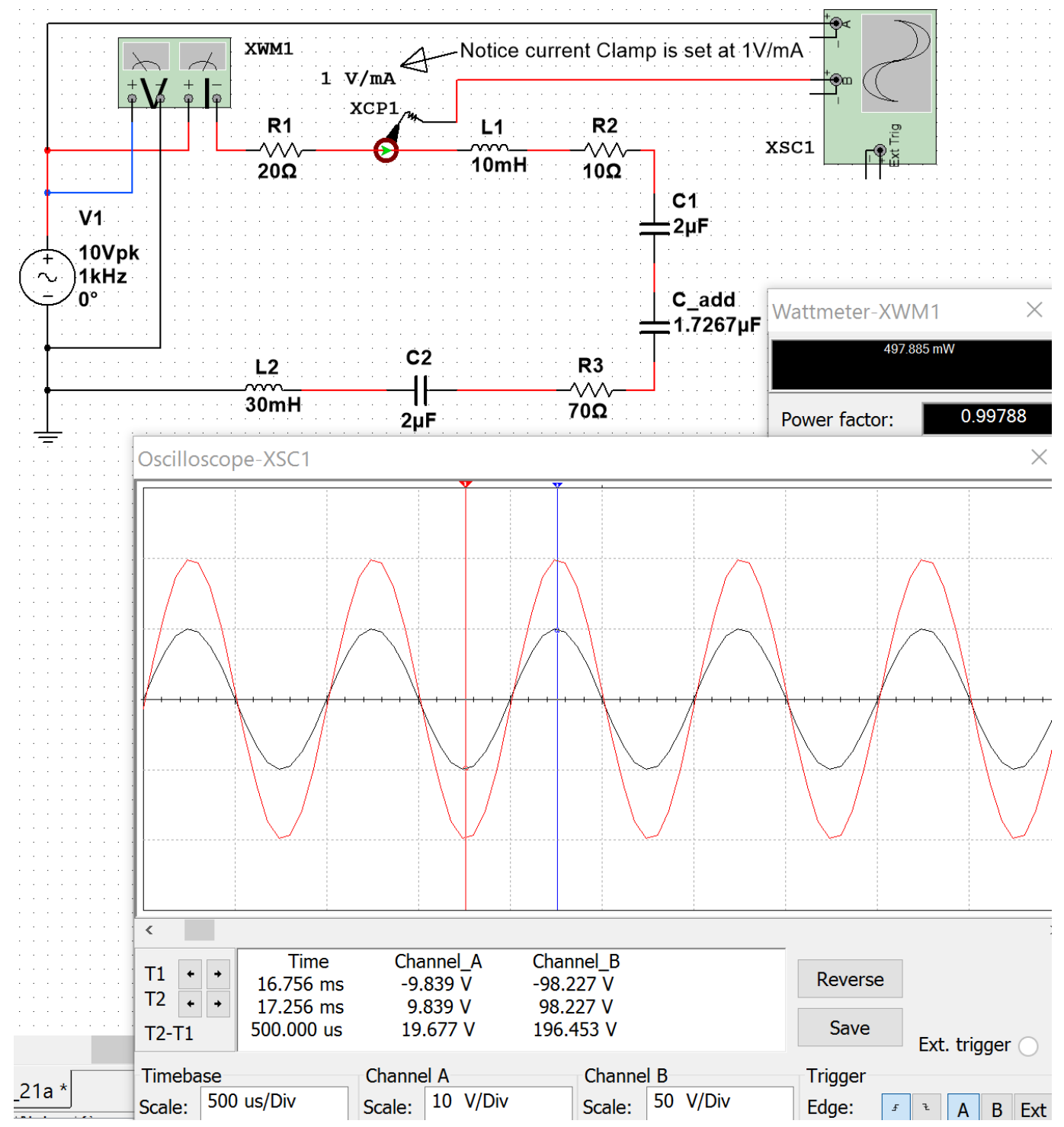

Figure 3.22: Verification that added capacitor makes power factor go to approximately 1. 
The power triangle for the circuit before the capacitor is added is shown in Figure 3.23.

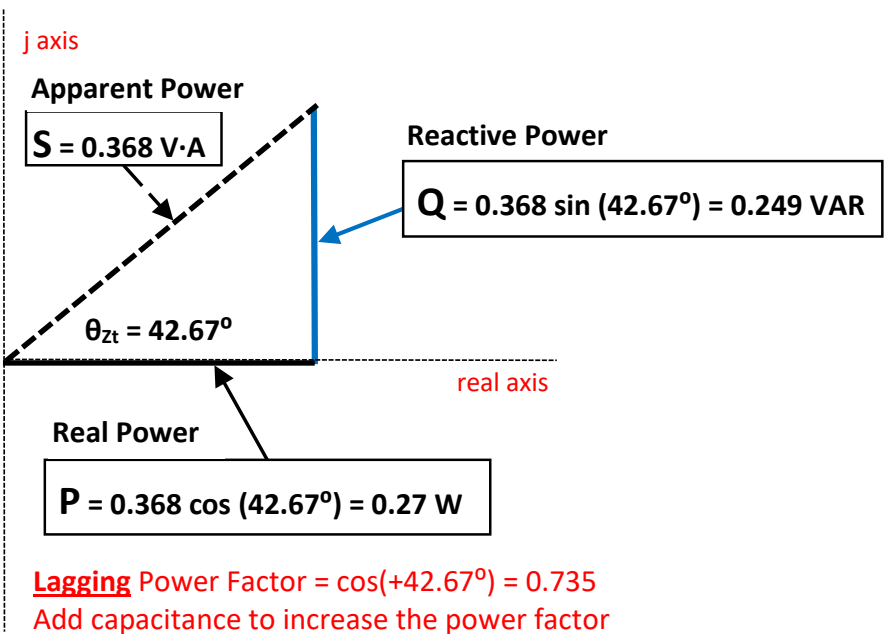

Figure 3.23: Power Triangle for the circuit in Example 3.9.

Example 3.9 is a very practical problem because power companies are constantly trying to make the power factor closer to 1 in their transmission lines. The load of the customers they supply electricity to is primarily inductive (due to motors) so the transmission line circuit is initially in quadrant I (ELI). The power companies purposely add large capacitors into the line to remove the VARs and increase the power factor closer to 1 . When the peak load ends when businesses shut down for the day the capacitors added to negate inductance might add too much reactance and move the total impedance into quadrant IV (ICE). When this happens the some of the capacitance added to the line is removed. This is a continual process that the power companies monitor to increase the power factor and save money by making the transmission line more efficient.

\section{Section 3.5 -AC Maximum Power Transfer}

Example 3.9 showed how to modify a circuit to increase the power factor so that more of the power that is generated is delivered to the load and less VAR waste occurs. This section contains a similar concept, but also factors in the resistance balancing that is needed to maximize power transfer. For DC circuits the Maximum Power Transfer Theorem stated that the maximum power transfer occurs when the load resistance is equal to the Thevenin resistance of the circuit where the load is connected. In Figure 3.24 it can be seen that as the value of $R_{L}$ is close to zero on the $x$-axis the power dissipated by $R_{L}$ is also close to zero, but when RL reaches $1000 \Omega$, which is the value of $R$ th, the power dissipated in $R_{L}$ is at its maximum.

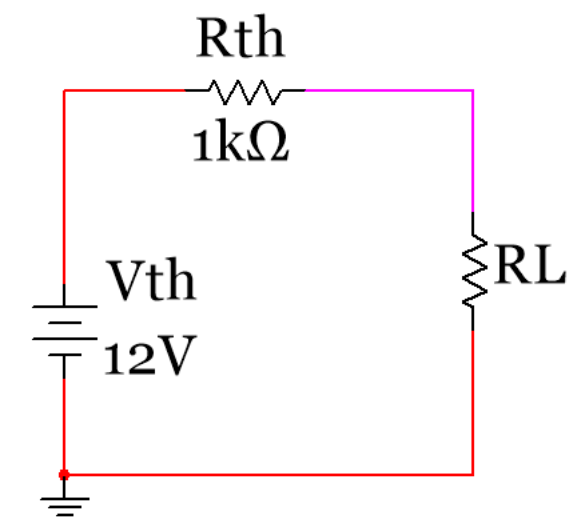

PRL versus $R L$

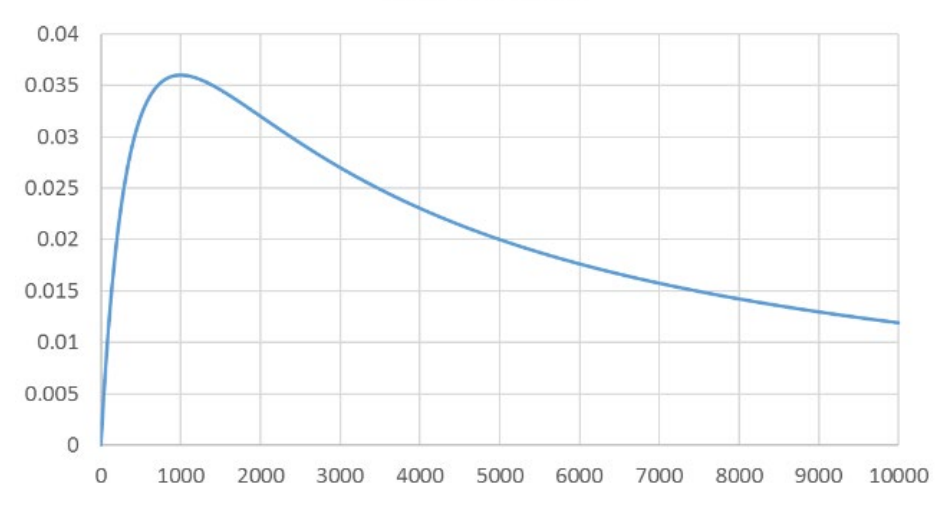

Figure 3.24: Graphical representation of the Maximum Transfer Theorem $\left(R_{L}=R\right.$ th $)$ for $D C$ circuits 
For AC circuits the theorem is modified to account for the resistance and the reactance.

\section{[3.6] $Z_{\text {Load }}=Z_{\text {Source }}^{*}$ (Maximum Power Transfer Theorem for AC circuits)}

The * in Equation 3.6 denotes the conjugate. The following two scenarios could occur:

1. You know the source impedance $\left(Z_{\text {source }}\right)$ and need to select a load impedance $\left(Z_{\text {Load }}\right)$ for maximum power transfer so you try to set it equal to the conjugate of the source.

2. You have a set load and need to modify the source for maximum power transfer so you try to make the source impedance equal to the conjugate. This is the scenario of Example 3.9.

Example 3.10) Determine the load impedance for maximum power transfer if the source impedance is equal to 3 $+j 4$. Use the A+jB Block and A-jB Block parts in Multisim that are located in the following directory: Group $\rightarrow$ Basic, Family $\rightarrow Z_{-}$Load. Solve the problem to determine the apparent, reactive, and real power.

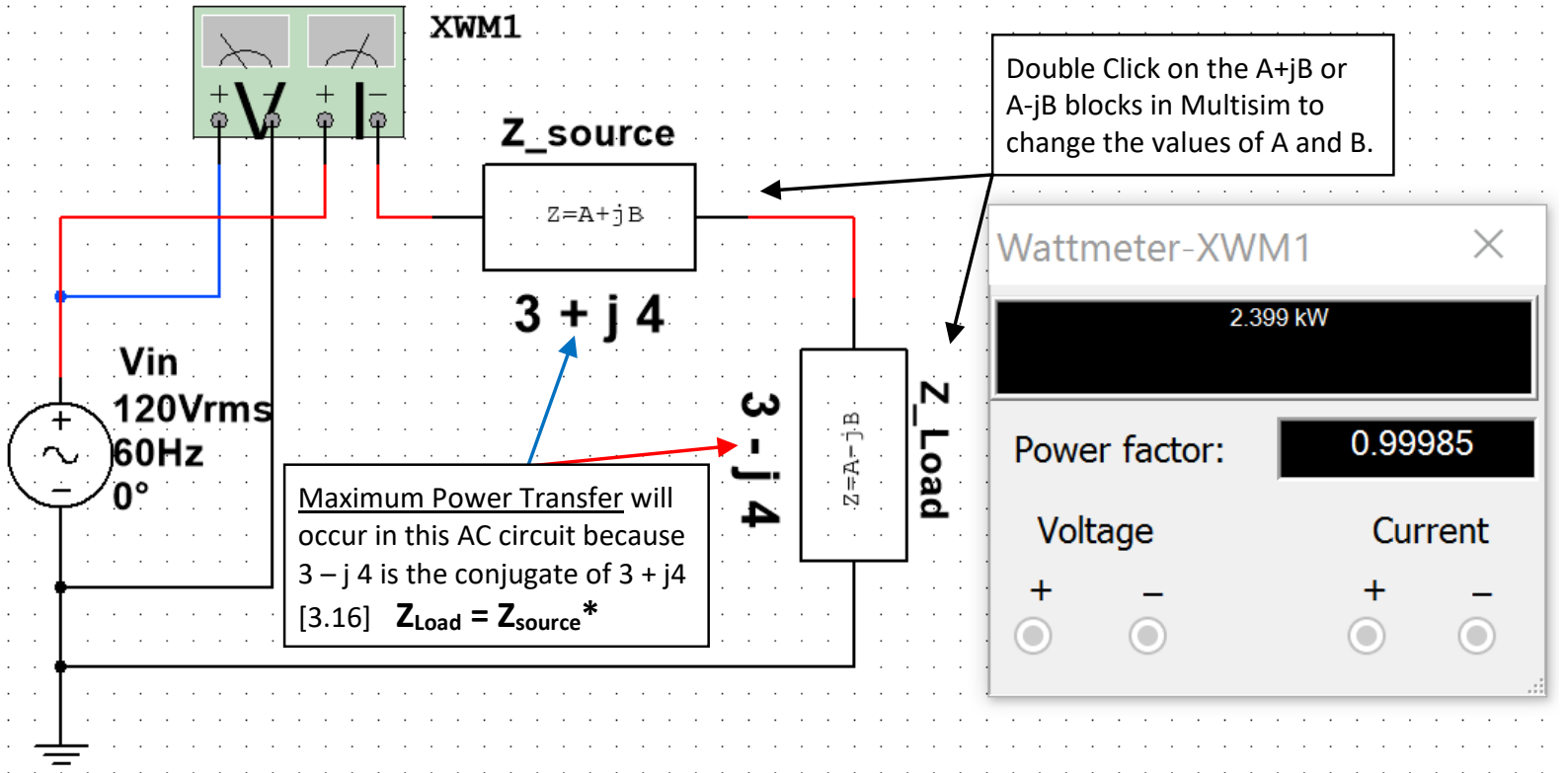

Figure 3.25: Circuit including Multisim Z_loads for Example 3.10.

- $\quad$ The Load impedance must be 3 - j 4 for maximum power transfer (as shown in Figure 3.25).

- Ztotal $=(3+\mathrm{j} 4)+(3-\mathrm{j} 4)=6 \Omega$

- $\mathrm{I}_{\mathrm{RMS}}=\mathrm{V}_{\mathrm{RMS}} /$ Ztotal $=\left(120 \angle 0^{\circ}\right) /\left(6 \angle 0^{\circ}\right)=20$ ARMS

- $\theta_{\mathrm{Zt}}=0^{\circ} \rightarrow \mathrm{PF}=\cos \left(0^{\circ}\right)=1$

- $S=I_{R M S} \cdot V_{R M S}=\left(20 A_{R M S}\right) \cdot\left(120 V_{R M S}\right)=\underline{2400 ~ V \cdot A}$

- $\mathrm{P}=\mathrm{S} \cdot \cos \left(0^{\circ}\right)=\underline{\mathbf{2 4 0 0} \mathbf{W}} \rightarrow$ Multisim has value equal to $\underline{2399 \mathrm{~W}}$

- $\mathrm{Q}=\mathrm{S} \cdot \sin \left(0^{\circ}\right)=\underline{\mathbf{O} \text { VAR }}$

The Z_load blocks made Example 3.10 easier. If given only the reactance of a capacitor or inductor instead of the actual value of them, Z_load blocks allow you to skip the step of calculating inductance or capacitance. For AC circuits with multiple impedances in series and parallel Z_loads simplify the problem in Multisim. 
Power transmisssion and distribution systems (as seen in Figure 3.26) is the most important application of RLC circuits. Examples that showed how impedance matching is performed to increase the amount of power delivered to the load was shown in examples 3.9 and 3.10. The following link contains a convenient interactive interface that solves for some of the AC parameters in a series RLC circuit. http://hyperphysics.phyastr.gsu.edu/hbase/electric/powerac.html

For deeper reading on transmission lines Chapter 14 of the AllAboutCircuits.com - Alternating Current eBook is recommended. http://www.allaboutcircuits.com/textbook/alternating-current/chpt-14/50-ohm-cable/

The following link also provides a brief overview of the electrical effects of the physical size of the transmission line. http://www.allaboutcircuits.com/technical-articles/introduction-to-the-transmission-line/

Color Key:

Red: Generation

Blue: Transmission

Green: Distribution

Black: Customer

Generating Station

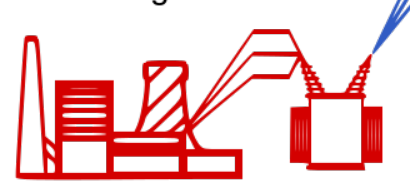

Generating Step Up Transformer
Transmission lines $765,500,345,230$, and $138 \mathrm{kV}$

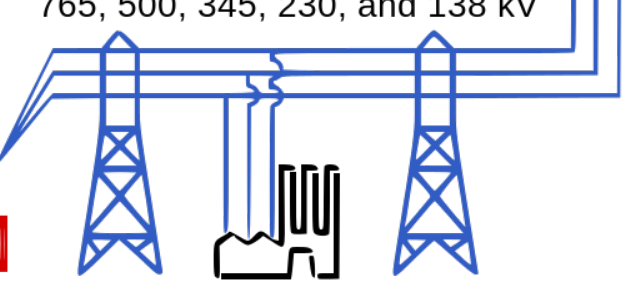

Transmission Customer $138 \mathrm{kV}$ or $230 \mathrm{kV}$
Substation Step Down Transformer

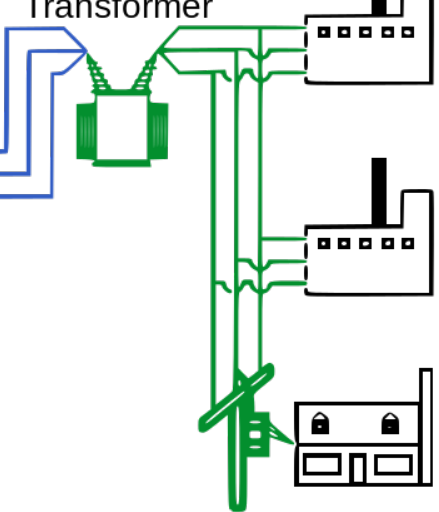

Subtransmission Customer $26 \mathrm{kV}$ and $69 \mathrm{kV}$

Primary Customer $13 \mathrm{kV}$ and $4 \mathrm{kV}$

Secondary Customer $120 \mathrm{~V}$ and $240 \mathrm{~V}$

Figure 3.26: Electrical Transmission System Overview. Source: Public domain image

The following link shows a figure with more information about the electrical grid and includes different sources of electrical generation such as: wind, solar, hydro-electric, nuclear, and coal.

\section{https://commons.wikimedia.org/wiki/File:Electricity Grid Schematic English.svg}


While power transmission is probably the most important application of RLC circuits, filtering is a close second. Chapter 8 of the AllAboutCircuits.com - Alternating Current eBook provides details about filters and is recommended reading to gain a deeper understanding about the subject.

http://www.allaboutcircuits.com/textbook/alternating-current/chpt-8/what-is-a-filter/

There are two main categories of filters: passive and active. Active filters require a power source for operation, but passive filters do not. Active filters include Operational Amplifiers (discussed in Module 7), while passive filters use inductors and/or capacitors to filter signals. In this eBook only passive filters are covered, but references for active filters are provided in Module 7 and a active filter example is shown in Figure A.4 in the appendix.

In this section some of the common RL, RC, and RLC passive filters are discussed and example circuits with calculations are provided to provide an overview of the topic. The frequency response tool called the Bode plot will be used to provide a graphical understanding of how filter work. Many applications have Bode plot function. In Matlab the function bode (or bodeplot) can be used to determine the magnitude response (how much the output magnitude changes from the input) and phase response (how much the output phase changes from the input) for a range of input frequencies. The Matlab function bodemag can also be used if only the magnitude response is needed. The value obtained from the Bode magnitude response will be referred to as the gain $\left(V_{\text {out }} / V_{\text {in }}\right)$ for the remainder of this document. Performing frequency analysis of circuits in Matlab with the Bode plot requires the circuit to be solved in the Laplace domain. An example of the Laplace solving method is shown for a Band Pass Filter in Figure 4.15 and also for Low Pass and High Pass filters in the Appendix in Figure A.1, Figure A.2, and Figure A.3.

Multisim also has a Bode plot tool that is very useful when simulating AC circuits. The tool is located 4 spots below the Multimeter on the right side of the Multisim window. To understand how to use the Multisim Bode plot Figure 4.1 shows a circuit with two resistors that form a voltage divider and reduces the output voltage by $50 \%$ (i.e. Gain $=0.5 \mathrm{~V} / \mathrm{V}$ ). The gain can also be represented in decibels (or $\mathrm{dB}$ ). Equation 4.1 shows how to convert from the "absolute" gain (which is in units of either V/V or A/A) to dB.

\section{[4.1] $\operatorname{Gain}_{d B}=20 \cdot \log _{10}\left(\operatorname{Gain}_{V / V}\right)$}

For example, the magnitude of the gain in Volt/Volt (or V/V) for the circuit in Figure 4.1 is calculated as:

- Voltage Divider Rule: $\mathrm{V}_{\text {out }}=\mathrm{V}_{\text {in }}[1 \mathrm{k} \Omega /(1 \mathrm{k} \Omega+1 \mathrm{k} \Omega)] \rightarrow \mathrm{G}_{V / V}=\frac{V_{\text {out }}}{V_{\text {in }}}=0.5 \frac{\mathrm{V}}{\mathrm{V}}$

- $\mathrm{G}_{d B}=20 \cdot \log _{10}\left(0.5 \frac{\mathrm{V}}{\mathrm{V}}\right)=\underline{\mathbf{6}} \mathbf{. 0 2 1 \mathrm { dB }}$

- Notice the Multisim Bode Plot cursor shows the magnitude is a constant value of $-6.021 \mathrm{~dB}$.

- Since it is known that resistors don't change the phase, the phase is verified in Figure 4.2 where the phase is shown to be $0^{\circ}$ for this circuit. This means that $0^{0}$ is added to the input phase to get the output phase. This process might seem pointless for this circuit because the phase doesn't affect the output, but it will be important for circuits that have a Bode plot with a non-zero phase change.

Equation 4.2 shows the output from the Bode plot given a specific input voltage, $v_{\text {in }}(t)=A \cdot \sin (\omega \cdot t+\theta)$.

[4.2] $v_{\text {out }}(\mathrm{t})=\mathrm{A} \cdot G_{V / V} \cdot \sin (\omega \cdot \mathrm{t}+\theta+\Delta \theta)$ Get $G_{V / V}$ and $\Delta \theta$ from the Bode plot at the input frequency. 
Example 4.1 shows how to apply Equation 4.2 to determine the output of a LPF circuit.

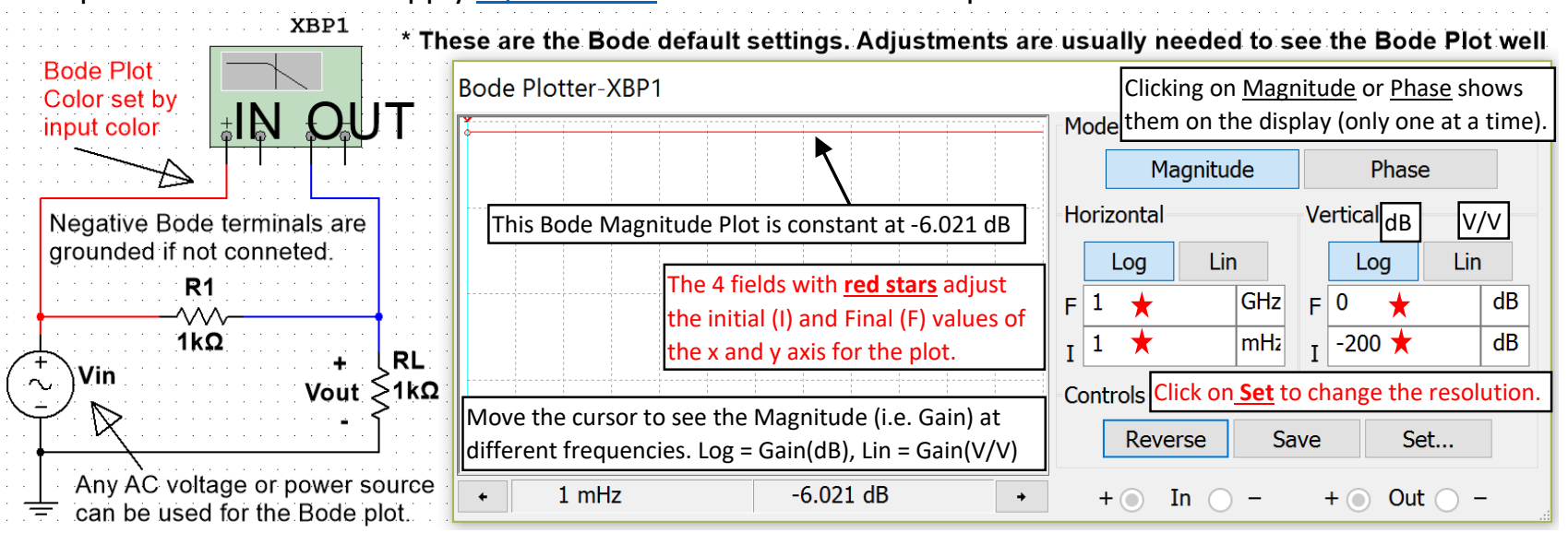

Figure 4.1: Multisim Bode Plot with the default settings.

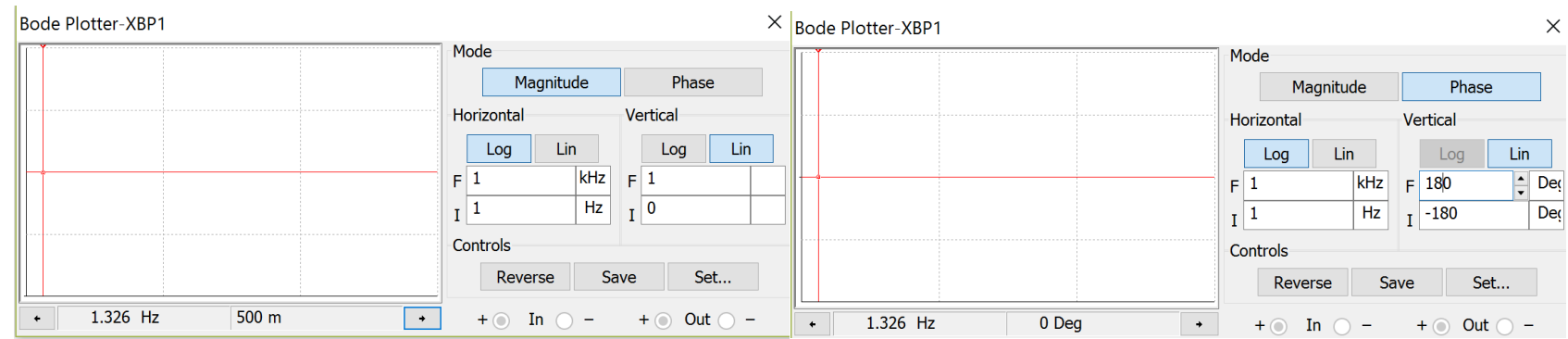

Figure 4.2: Bode Plot from Figure 4.1 with adjustments: Magnitude Response (Left), Phase Response (Right).

The cursors are set in Figure 4.2 at $1.326 \mathrm{~Hz}$ for both the magnitude (left) and phase (right) modes. At $1.326 \mathrm{~Hz}$ the gain is listed as " $500 \mathrm{~m}$ " (or $0.5 \mathrm{~V} / \mathrm{V}$ ) and the phase is $0^{\circ}$. It is apparent from the bode plot that both the gain and phase are constant so the cursors for this circuit will result in $500 \mathrm{~m}$ and $0^{\circ}$ no matter what the frequency value of the input signal. However, for circuits with inductors or capacitors both the gain and phase will change as the frequency changes so changing the frequency limits, resolution, and cursor position are needed to determine the gain and phase at the desired frequency. Applying the values in the Bode plot in Figure 4.2 to the following input signal at a frequency of $1.326 \mathrm{~Hz} \rightarrow v_{\text {in }}(\mathrm{t})=3 \cdot \sin (2 \pi \cdot 1.326 \cdot \mathrm{t})$

Using Equation 4.2, the following result is obtained for the output signal from the circuit in Figure 4.1.

$$
\text { - } v_{\text {out }}(\mathrm{t})=\mathrm{A} \cdot G_{\bar{V}} \cdot \sin (\omega \cdot \mathrm{t}+\theta+\Delta \theta)=3 \cdot 0.5 \cdot \sin \left(2 \pi \cdot 1.326 \cdot \mathrm{t}+0^{0}\right)=\mathbf{1 . 5} \cdot \sin (\mathbf{2 \pi} \cdot \mathbf{1} \cdot \mathbf{3 2 6} \cdot \mathrm{t})
$$

\section{Section 4.1 - Low Pass Filters (LPF)}

In general terms, a low pass filter (LPF) allows input signals with lower frequencies to pass through to the output while attenuating (i.e. reducing the amplitude) input signals with higher frequencies. The circuits in Figures 4.3 through 4.5 are called $\underline{1}^{\text {st }}$ order LPF circuits because they only have one reactive component (i.e. capacitor or inductor). Only $1^{\text {st }}$ order LPF circuits will be discussed in this section. Figure A.2 in the appendix shows how to solve higher order filter circuits using the Laplace solving method. The capacitor or inductor in the $1^{\text {st }}$ order LPF causes a $3 \mathrm{~dB}$ frequency $\left(\mathrm{f}_{3 \mathrm{~dB}}\right)$, which is the point at which the slope of the Bode magnitude plot changes by 20

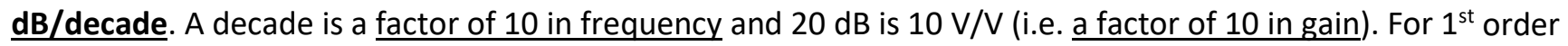
LPF circuits, the reactive component causes the slope of the Bode magnitude plot to gradually change from flat $(0 \mathrm{~dB} / \mathrm{dec})$ to $-20 \mathrm{~dB} / \mathrm{dec}$ at $\mathrm{f}_{3 \mathrm{~dB}}$. The $3 \mathrm{~dB}$ frequency can either be denoted as angular frequency $\left(\omega_{3 \mathrm{~dB}}\right)$ or cyclic frequency $\left(f_{3 \mathrm{~dB}}\right)$. $\omega_{3 \mathrm{~dB}}$ is equal to the inverse of the time constant $(\tau)$ for a $1^{\text {st }}$ order LPF or HPF. The reason the 
$3 \mathrm{~dB}$ subscript is used is due to the gain in the Bode magnitude plot being $3 \mathrm{~dB}$ below the "passband" at $\mathrm{f}_{3 \mathrm{~dB}}$ as shown in Figures 4.3 through 4.7. The pass band is the flat region of the Bode magnitude plot that corresponds to the frequency range where the gain is at its highest value. Over the pass band frequency range the Bode phase plot approaches $0^{\circ}$ for RLC circuits and non-inverting OpAmp circuits and approaches $180^{\circ}$ for inverting OpAmp circuits. Operational Amplifiers (OpAmps) are discussed in Module 7. For RLC circuits each inductor or capacitor causes a $\pm 90^{\circ}$ phase change in the Bode phase plot. The part of the frequency response that is not in the pass band is called the stop band.

[4.3] $3 d B$ Angular Freqeuncy $=\omega_{3 d B}=\frac{1}{\tau} *$ For a $1^{\text {st }}$ order RL or RC circuit
[4.4] $3 d B$ Cyclic Freqeuncy $=f_{3 d B}=\frac{1}{2 \pi \cdot \tau} \quad *$ For a $1^{\text {st }}$ order RL or RC circuit

[4.5] Capacitor Time Constant $(\tau)=\tau_{C}=R_{e q} C$

[4.6] Inductor Time Constant $(\tau)=\tau_{L}=\frac{L}{R_{e q}}$

- $\mathrm{R}_{\mathrm{eq}}$ is the equivalent resistance "seen" by the capacitor or inductor.

In DC circuits the time constant $(\tau)$ for capacitors and inductors was used for transient analysis of DC circuits in the decaying term $\rightarrow e^{-t / \tau}$. The larger the time constant the longer it took to charge the voltage of a capacitor or to charge the current in an inductor. For filtering, a larger the time constant results in a smaller $3 \mathrm{~dB}$ frequency.

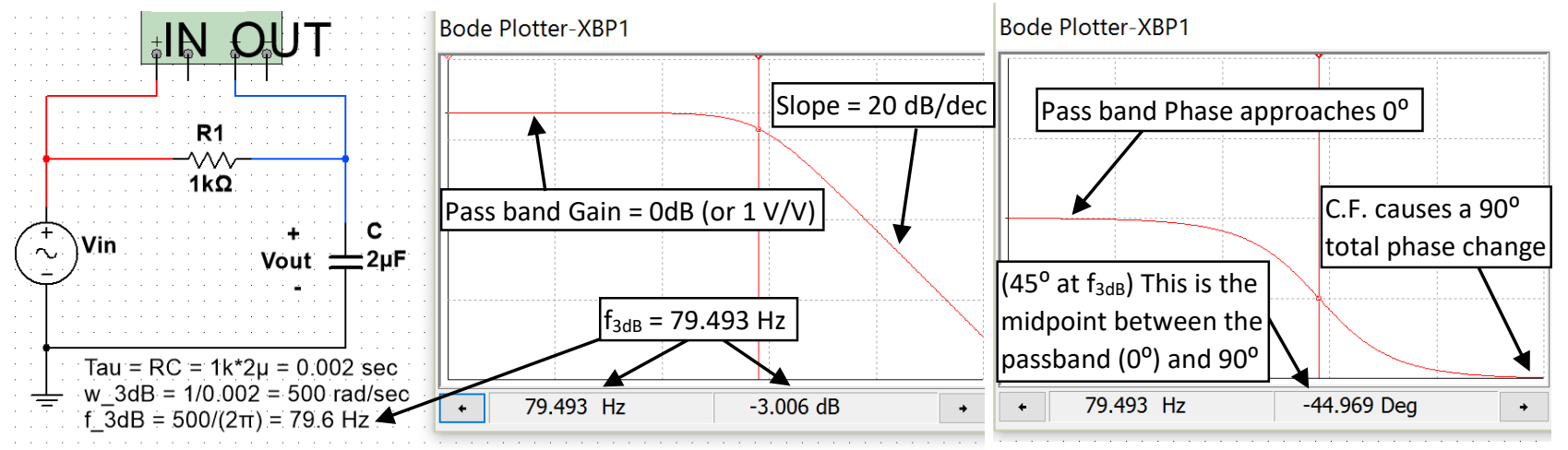

Figure 4.3: $1^{\text {st }}$ Order RC Low Pass Filter Circuit with a $3 \mathrm{~dB}$ frequency $\left(f_{3 \mathrm{~dB}}\right)$ calculated to equal $79.6 \mathrm{~Hz}$.

Figure 4.3 shows the $3 \mathrm{~dB}$ frequency is calculated at $79.6 \mathrm{~Hz}$ and the gain is $3 \mathrm{~dB}$ below the pass band gain at that frequency. Since the output is usually desired to be a resistive load, a common variant of the RC filter in Figure $\underline{4.3}$ is shown in Figure 4.4. Recall that a capacitor acts as an open circuit at DC, so the steady state DC output for the circuit in Figure 4.4 is equal to $0.5 \cdot V_{\text {in }}$ using the same voltage divider calculation that was used for the circuit in Figure 4.1. With the pass band gain at $0.5 \mathrm{~V} / \mathrm{V}$ (or $-6 \mathrm{~dB}$ ) the gain at $\mathrm{f}_{3 \mathrm{~dB}}$ is equal to $-9 \mathrm{~dB}(3 \mathrm{~dB}$ below $-6 \mathrm{~dB})$ as shown below. 


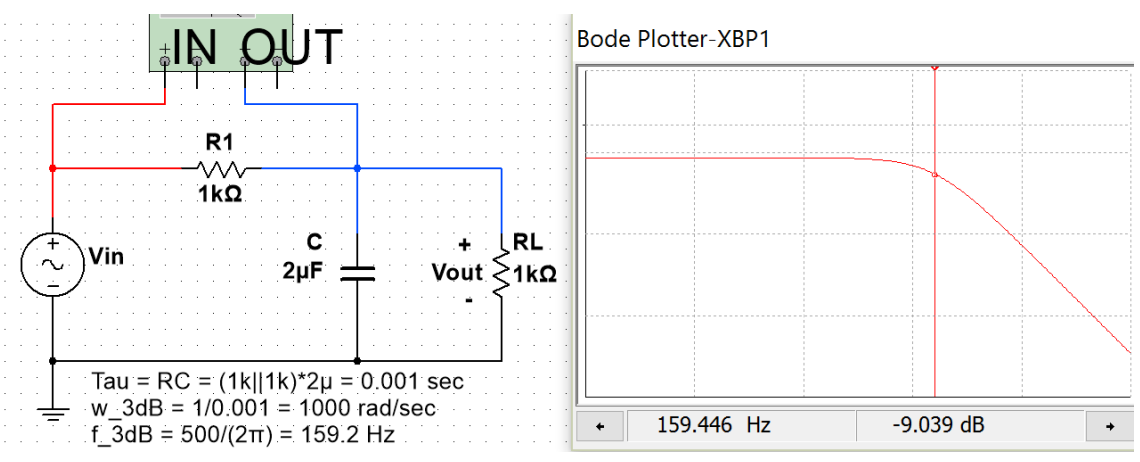

Figure 4.4: $1^{\text {st }}$ Order RC Low Pass Filter Circuit with the output across a resistor.

Next, an RL LPF is shown in Figure 4.5. Recall that an inductor acts as a short circuit at DC steady state, so the DC voltage will not be reduced due to an inductor being in series between the output and the input. In other words, the inductor will act like a short circuit at $D C$ and $V_{\text {out }}$ will equal $V_{\text {in. }}$ So at $D C$ (or $0 \mathrm{~Hz}$ ), which is the far left of the $\mathrm{x}$-axis of the Bode plot, the gain will be equal to $1 \mathrm{~V} / \mathrm{V}$ (or $0 \mathrm{~dB}$ ) just like it was in the $1^{\text {st }}$ order RC filter with one resistor (see Figure 4.3). Since it is a $1^{\text {st }}$ order filter and therefore only has one reactive component (the inductor) the slope of the Bode magnitude plot will be equal to $(20 \mathrm{~dB} / \mathrm{dec})$ as it was for the circuit in Figure 4.3. The Bode phase response plot is also the same as the RC LPF circuit in Figure 4.3.

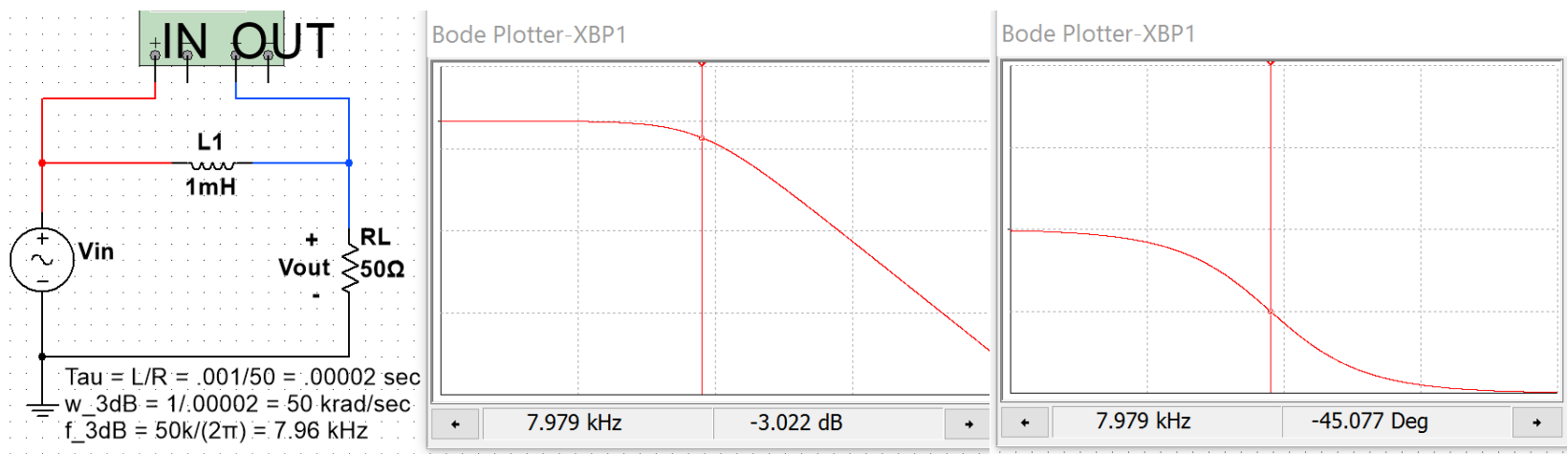

Figure 4.5: $\mathbf{1}^{\text {st }}$ Order RL Low Pass Filter Circuit with a $3 \mathrm{~dB}$ frequency $\left(\mathrm{f}_{3 \mathrm{~dB}}\right)$ equal to $7.96 \mathrm{kHz}$.

Example 4.1) Determine the output, $v_{\text {out }}(t)$, from the circuit in Figure 4.5 for each of the following inputs:

- $v_{\text {in } 1}(t)=5 \sin (2 \pi \cdot 7.96 \cdot t)$

- $v_{\text {in } 2}(t)=120 \sin \left(2 \pi \cdot 0.796 \cdot t+20^{\circ}\right)$

- $v_{\text {in } 3}(t)=0.32 \sin \left(2 \pi \cdot 79.6 \cdot t-150^{\circ}\right)$

For each of the inputs the freqency is used to determine the magnitude and phase change from the Bode plot.

- $v_{\text {in1 } 1}(t)$ is at $f_{\text {in }}=7.96 \mathrm{~Hz}$. The Bode plot shows at $f_{\text {in }} \rightarrow \Delta \theta=\underline{-45^{\circ}}$ and $G_{d B}=-3 d B \rightarrow G_{v / N}=\underline{0.7071}$

- $v_{\text {out } 1}(\mathrm{t})=\mathrm{A} \cdot G_{V / V} \cdot \sin (\omega \cdot \mathrm{t}+\theta+\Delta \theta)=5 \cdot \mathbf{0 . 7 0 7 1} \cdot \sin \left(2 \pi(7.96) \cdot \mathrm{t}+0^{0}-\mathbf{4 5}^{0}\right)$

- $v_{\text {out } 1}(\mathrm{t})=3.54 \sin \left(15.92 \pi \cdot \mathrm{t}-45^{\circ}\right)$

- $\quad V_{\text {in2 } 2}(t)$ is at $f_{\text {in }}=0.796 \mathrm{~Hz}$. The Bode magnitude plot shows that 1 decade less than $f_{3 d B}$ the gain is safely in the passband. With only 1 resistor, the gain should be $\sim 0 \mathrm{~dB}$ (or $1 \mathrm{~V} / \mathrm{V}$ ) and $\Delta \theta$ should be $\sim 0^{\circ}$.

- $v_{\text {out } 2}(\mathrm{t})=\mathrm{A} \cdot G_{V / V} \cdot \sin (\omega \cdot \mathrm{t}+\theta+\Delta \theta)=120 \cdot \mathbf{1} \cdot \sin \left(2 \pi(0.796) \cdot \mathrm{t}+20^{0}+\mathbf{0}^{0}\right)$

- $v_{\text {out } 2}(\mathrm{t})=120 \sin \left(1.592 \pi \cdot \mathrm{t}+20^{0}\right)=v_{\text {in2 }}(\mathrm{t})$

- $\quad V_{\text {in3 }}(t)$ is at $f_{\text {in }}=79.6 \mathrm{~Hz}$ which is 1 decade greater than $f_{3 d B}$. For a $1^{\text {st }}$ order LPF the gain drops by $20 \mathrm{~dB}$ in 1 decade due to the reacive component so the gain is $\sim-23 \mathrm{~dB}$ (or $0.07071 \mathrm{~V} / \mathrm{V}$ ) and $\Delta \theta$ is $\sim-90^{\circ}$.

- $v_{\text {out } 3}(\mathrm{t})=\mathrm{A} \cdot G_{V / V} \cdot \sin (\omega \cdot \mathrm{t}+\theta+\Delta \theta)=0.32 \cdot \mathbf{0 . 0 7 0 7 1} \cdot \sin \left(2 \pi(79.6) \cdot \mathrm{t}-150^{0}-\mathbf{9 0}^{0}\right)$

- $v_{\text {out } 3}(t)=0.02265 \sin \left(159.2 \pi \cdot t-240^{\circ}\right)=0.02265 \sin \left(159.2 \pi \cdot t+120^{0}\right)$

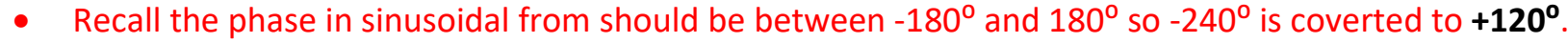


Note: A $2^{\text {nd }}$ order LPF is solved with Matlab using the Laplace Transform method in Figure A.2 in the Appendix.

\section{Section 4.2 - High Pass Filters (HPF)}

In general terms, a high pass filter (HPF) acts in opposite fashion as a LPF where it allows input signals with higher frequencies to pass through to the output while attenuating (i.e. reducing the amplitude) input signals with lower frequencies. The cirucit in Figure 4.6 is the most common type of High Pass Filter (HPF). Anytime a capacitor is placed in series in between the input and output a HPF is produced. When a capacitor is placed in this fashion it is often called a coupling capacitor or a DC blocking capacitor because the capacitor blocks the DC signal and prevents it from getting to the output. The gain at $0 \mathrm{~Hz}$ is $0 \mathrm{~V} / \mathrm{V}$ so the far left side of the Bode magnitude plot starts low and ramps up at $20 \mathrm{~dB} / \mathrm{dec}$ until it reaches the $3 \mathrm{~dB}$ frequency. Then (at $\mathrm{f}_{3 \mathrm{~dB}}$ ) the slope is gradually reduced by $20 \mathrm{~dB} / \mathrm{dec}$ resulting in a flat response $(0 \mathrm{~dB} / \mathrm{dec})$.

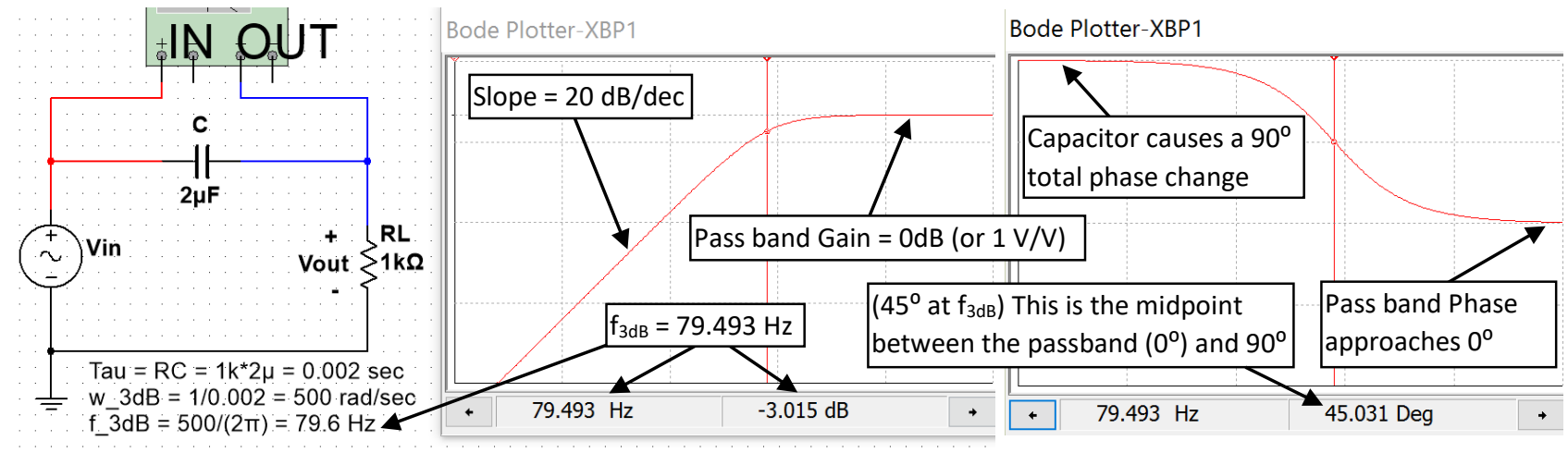

Figure 4.6: RC High Pass Filter Circuit with a $3 \mathrm{~dB}$ frequency $\left(f_{3 \mathrm{~dB}}\right)$ equal to $79.6 \mathrm{~Hz}$.

The other $1^{\text {st }}$ order filter with only one resistor is an RL circuit with the output taken across the inductor as shown in Figure 4.7.
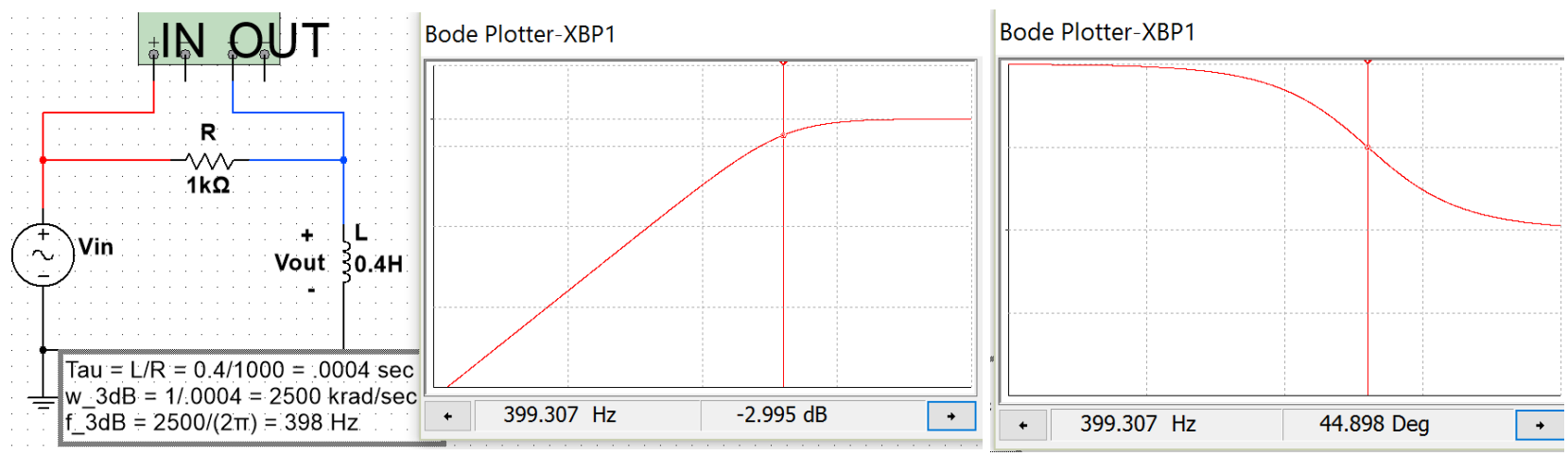

Figure 4.7: RL High Pass Filter Circuit with a $3 d B$ frequency $\left(f_{3 d B}\right)$ equal to $398 \mathrm{~Hz}$.

Example 4.2) Design a RC HPF circuit that has a $3 \mathrm{~dB}$ frequency of $1 \mathrm{kHz}$ and determine the output if the standard $A C$ wall outlet signal (equation shown below) is the input and the output is connected to a $10 \Omega$ load resistance.

- $\quad v_{\text {in } 1}(t)=120 \sqrt{2} \sin (2 \pi \cdot 60 \cdot t)$

To achieve a $f_{3 d B}$ frequency of $1 \mathrm{kHz}$ Equation 4.4 shows the time constant is equal to $1 /(2 \pi \cdot 1000)=0.159 \mathrm{msec}$. Equation 4.5 shows that the time constant for a $\mathrm{RC}$ circuit is $\tau=\mathrm{Req}_{\mathrm{e}} \mathrm{C}$, where $\mathrm{R}_{\mathrm{eq}}$ is equal to the resistor in the circuit. To avoid reducing the gain only one resistor will be used in the circuit. $\mathrm{C}$ can be solved for using the 
equation $C=\tau / R_{\text {eq }}=0.000159 / 100=1.59 \mu \mathrm{F}$. This HPF circuit is shown below and the Bode magnitude plot in Multisim verifies that the gain is approximately $-3 \mathrm{~dB}$ at $1 \mathrm{kHz}$.
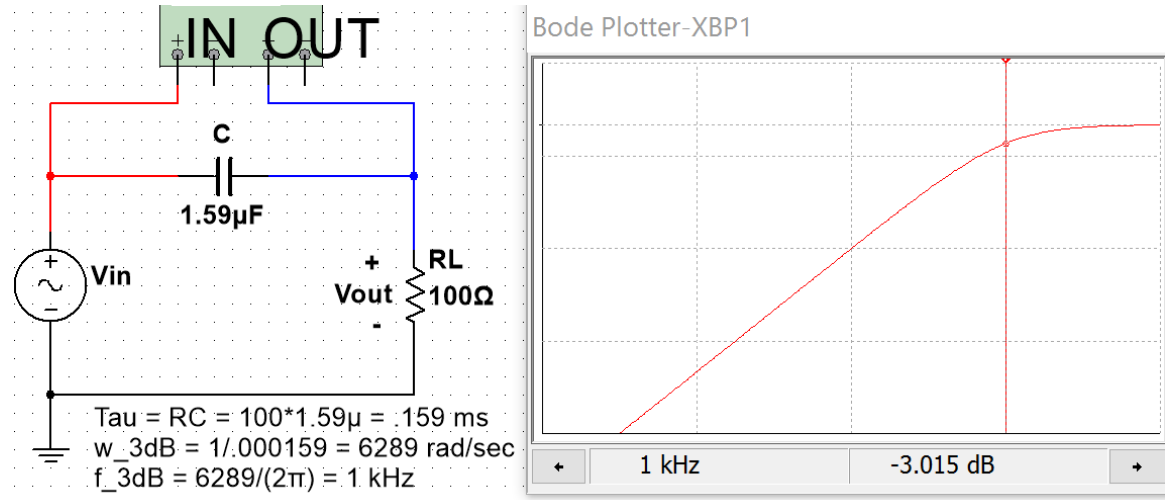

Figure 4.8: RC HPF design for Example 4.2 with Multisim verification of the $1 \mathrm{kHz} \mathrm{f}_{3 \mathrm{~dB}}$.

To determine the output from the AC wall outlet signal the gain and phase at $60 \mathrm{~Hz}$ are shown in Figure 4.9.

Bode Plotter-XBP1

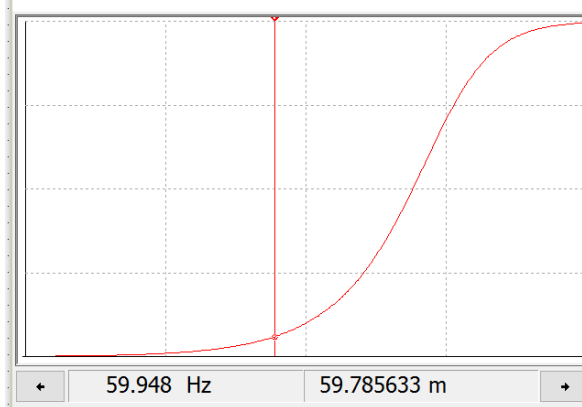

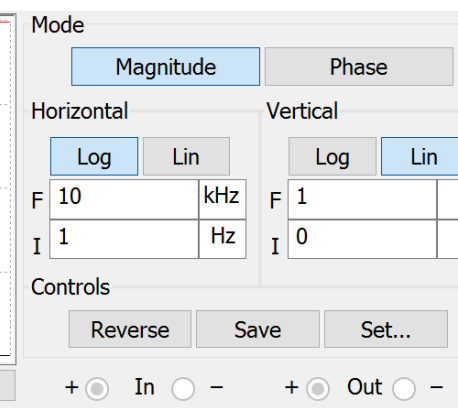

Figure 4.9: RC HPF design for Example 4.2 with Multisim gain and phase change at $60 \mathrm{~Hz}$.

The "Lin" option for the Bode magnitude plot is used in Figure 4.9 to avoid having to manually do the dB to V/V

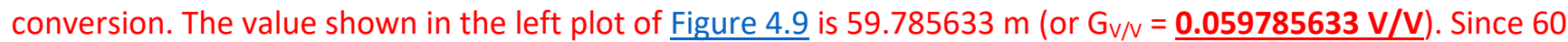
$\mathrm{Hz}$ is less than 1 decade away from the $1 \mathrm{kHz} \mathrm{f}_{3 \mathrm{~dB}}$ the phase change should be approximately $90^{\circ}$ different from the passband. To be more exact, we will use the cursor value at a frequency as close to $60 \mathrm{~Hz}$ as possible on the Bode phase plot (recall the resolution of the Bode plot is limited so exactly $60 \mathrm{~Hz}$ can't be selected). The value of the phase change $(\Delta \theta)$ in the right plot of Figure 4.9 is equal to $\underline{86.572^{\circ}}$.

- $v_{\text {out }}(\mathrm{t})=\mathrm{A} \cdot G_{V / V} \cdot \sin (\omega \cdot \mathrm{t}+\theta+\Delta \theta)=169.7 \cdot 0.0597856 \cdot \sin \left(2 \pi(60) \cdot \mathrm{t}+0^{0}+86.572^{0}\right)$

- $v_{\text {out } 1}(t)=10.15 \sin \left(120 \pi \cdot t+86.572^{0}\right)$

Using the Bode plot to determine the output signal is much easier than solving the problem algebraically. The following steps show how to solve this problem and the values are verified in the Multsim scope plot in Figure 4.10. The problem solving technique shown below is the same process that was performed in Section 3.2 - Series

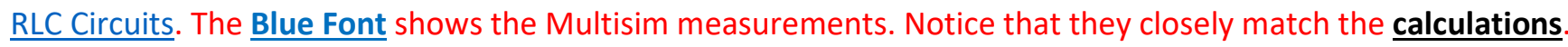

- $\omega=2 \pi \cdot f=2 \pi \cdot 60=\underline{377 \mathrm{rad} / \mathrm{sec}}$.

- $X_{C}=1 /(1.59 \mu \mathrm{F} \cdot \omega)=\underline{1668.3 \Omega}$

- $\mathrm{Z}_{\text {Total }}=\mathrm{RL}-\mathrm{j} \mathrm{X}_{\mathrm{C}}=100-\mathrm{j} \underline{1668.3} \mathrm{Z}_{\text {Total }}=\underline{\mathbf{1 6 7 1 . 3} \angle-86.57^{\circ} \Omega}$ (ICE) (Notice: $\mathrm{V}_{\mathrm{RL}}$ leads Vin in Figure 4.10)

- $\quad \mathrm{I}=\mathrm{Vin} / \mathrm{Z}_{\text {Total }}=169.7 \angle 0^{\circ} / 1671.3 \angle-86.57^{\circ}=\underline{\mathbf{1 0 1}} . \mathbf{5} \angle+\mathbf{8 6 . 5 7 ^ { 0 } \mathrm { mA }}$

- Time shift, $\mathrm{t}_{\mathrm{s},}$ of current $=-\theta / \omega=-86.57^{\circ} \cdot\left(\pi / 180^{\circ}\right) / 377 \mathrm{rad} / \mathrm{sec}=\underline{\mathbf{0 4}} .01 \mathrm{~ms}$ (-4.017 ms in Multisim)

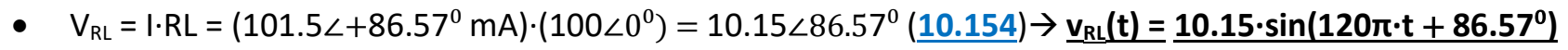



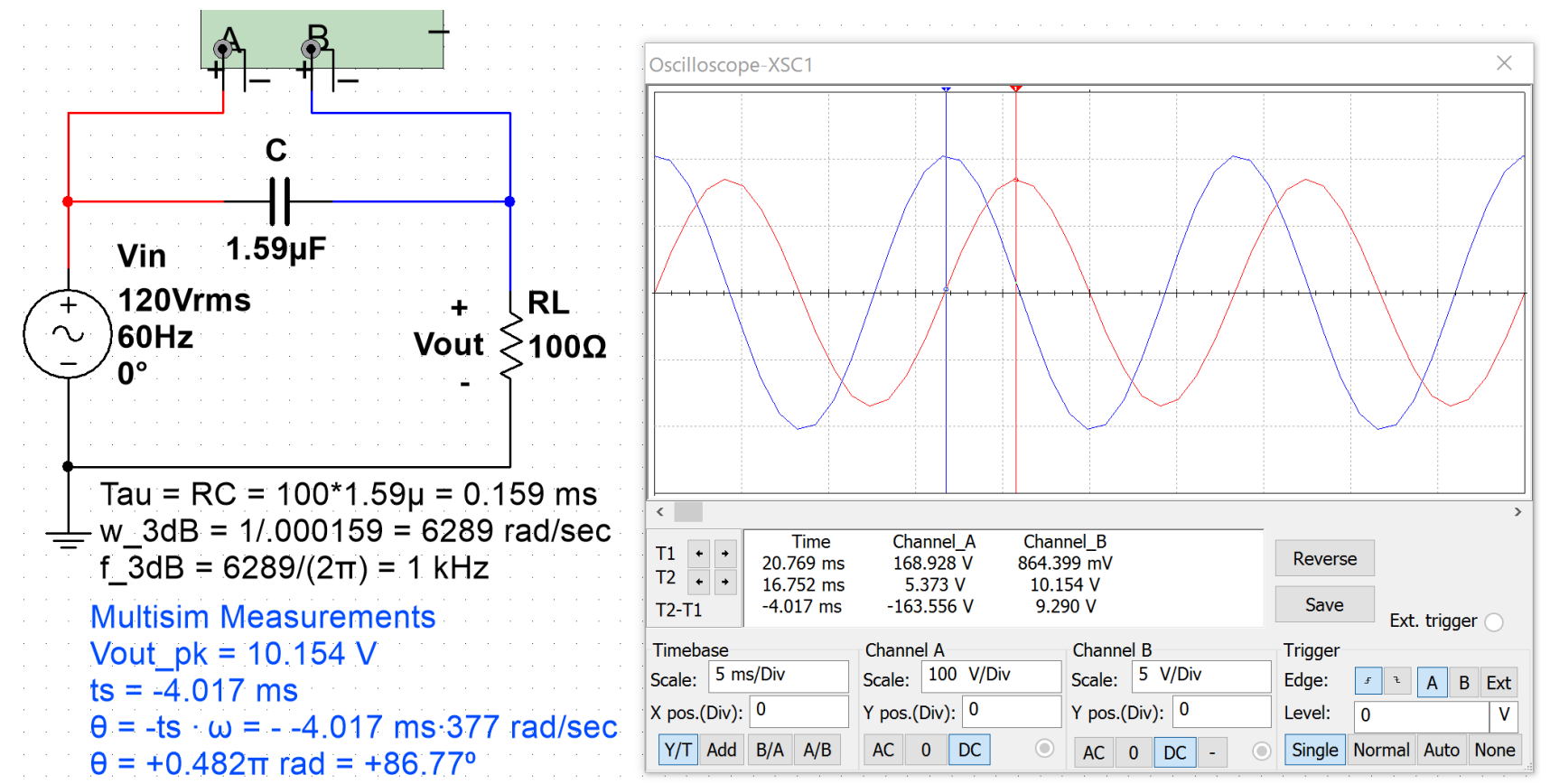

Figure 4.10: Multisim oscilloscope verification of Bode plot and calculated results of Example 4.2.

A $2^{\text {nd }}$ order HPF is solved with Matlab using the Laplace Transform method in Figure A.3 in the Appendix.

\section{Section 4.3-Band Pass Filters (BPF)}

In general terms, High Pass Filters "partially reject" signals with frequencies below $\mathrm{f}_{3 \mathrm{~dB}}$ and Low Pass Filters "partially reject" signals with frequencies above $f_{3 \mathrm{~dB}}$. The word "partially" is used because complete rejection would mean that an input signal at a specific sinusoidal frequency is multiplied by zero to completely remove it (and that doesn't occur). However, the further away the input signal's frequency is from $\mathrm{f}_{3 \mathrm{~dB}}$ the more rejection (or attenuation) occurs (i.e. the amplitude is multiplied by a value that approaches zero). At this point, before discussing Band Pass and Band Stop filters the terms "Pass Band" and "Stop Band" need to be reviewed.

- Pass Band - Section of the frequency response (or Bode magnitude plot) that is "passed".

- Stop Band - Section of the frequency response (or Bode magnitude plot) that is "rejected".

Figure 4.11 and Figure 4.12 show the Bode magnitude plots for the 4 categories of filters that are commonly used. Figure 4.11 shows the pass band and stop band for $1^{\text {st }}$ order Low Pass and High Pass filters. Figure 4.12 shows the pass band and stop band for the $2^{\text {nd }}$ order Band Pass Filter circuit in Figure 4.13 and the pass band and stop band for $2^{\text {nd }}$ order Band Stop Filter for the circuit in Figure 4.16. Only the most basic LPF, HPF, BPF, and BSF will be discussed in this eBook. Multiple capacitors and inductors can be arranged to produce higher order passive filters that have steeper Bode magnitude plots and therefore attenuate the signals at frequencies in the stop band to a greater extent (i.e. make the gain closer to $0 \mathrm{~V} / \mathrm{V}$ ). 

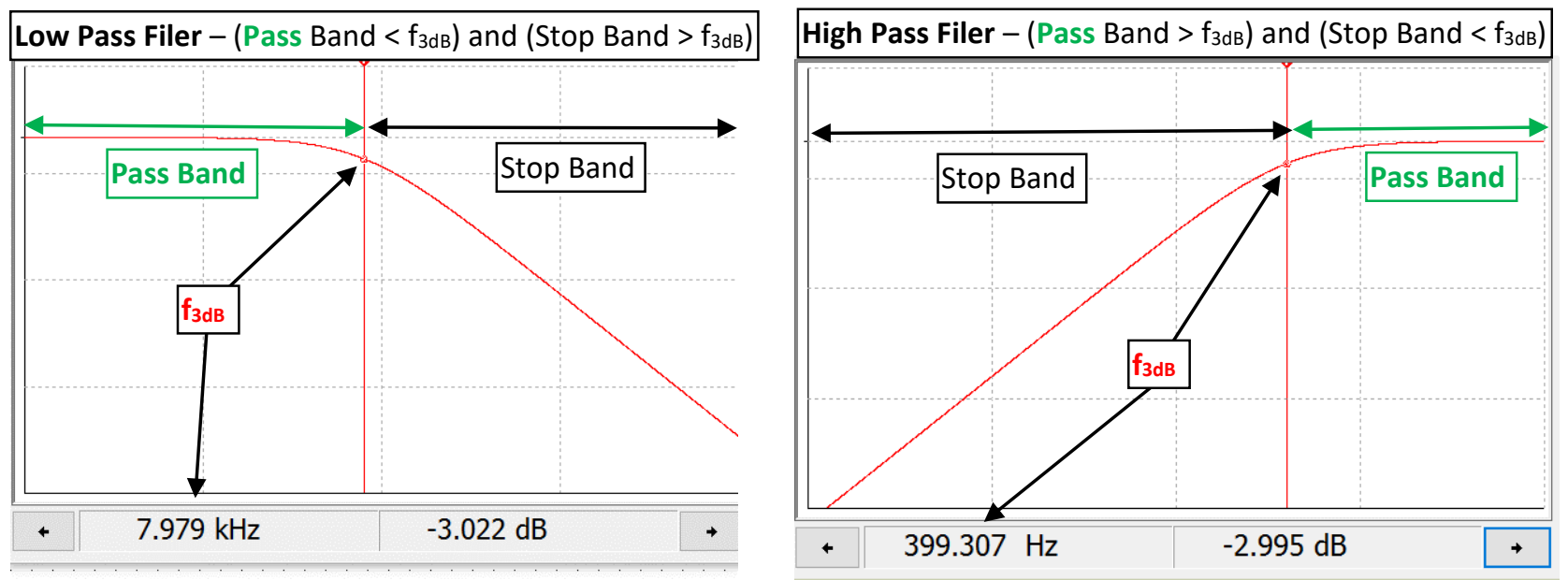

Figure 4.11: $1^{\text {st }}$ order LPF from circuit in Figure 4.5 (Left). $1^{\text {st }}$ order HPF from circuit in Figure 4.7 (Right)

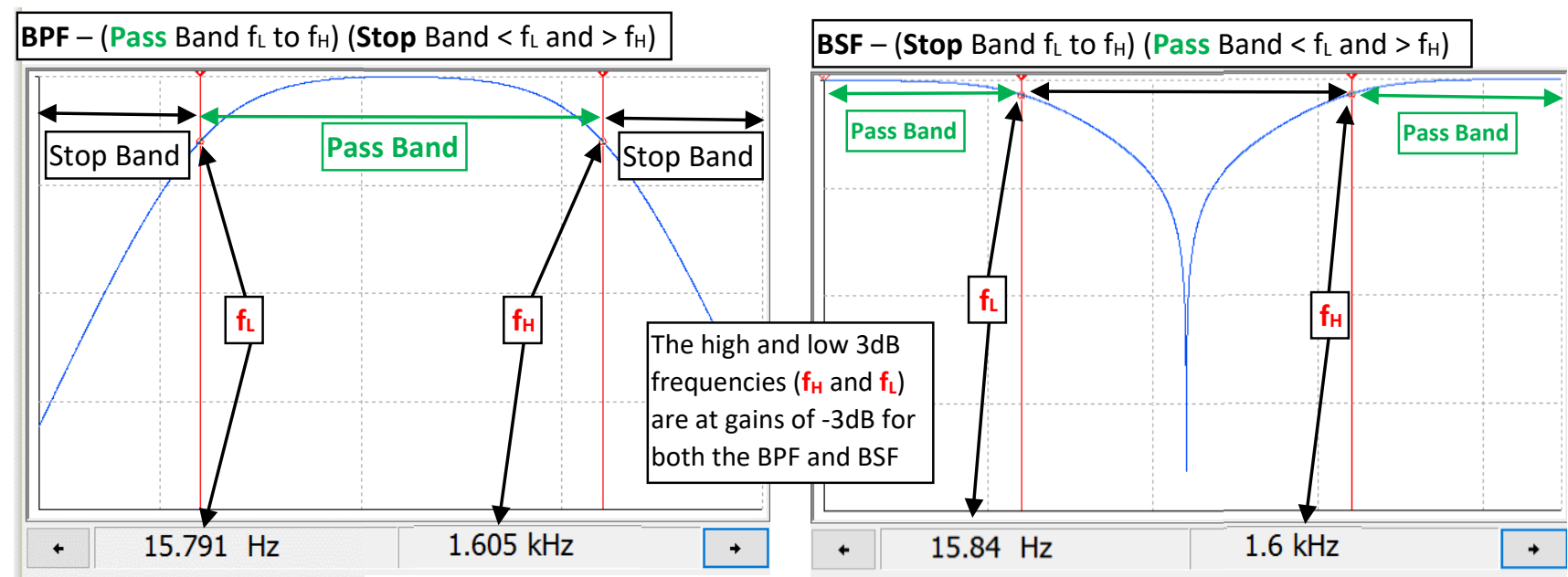

Figure 4.12: $2^{\text {nd }}$ order BPF from circuit in Figure 4.13 (Left). $2^{\text {nd }}$ order BSF from circuit in Figure 4.16 (Right)

By combining the RL LPF in Figure 4.5 and the RC HPF in Figure 4.6 a RLC BPF can be created as shown in Figure 4.13. The values of the components in the circuit give it a wide pass band which makes it a "Low- $Q$ " BPF.
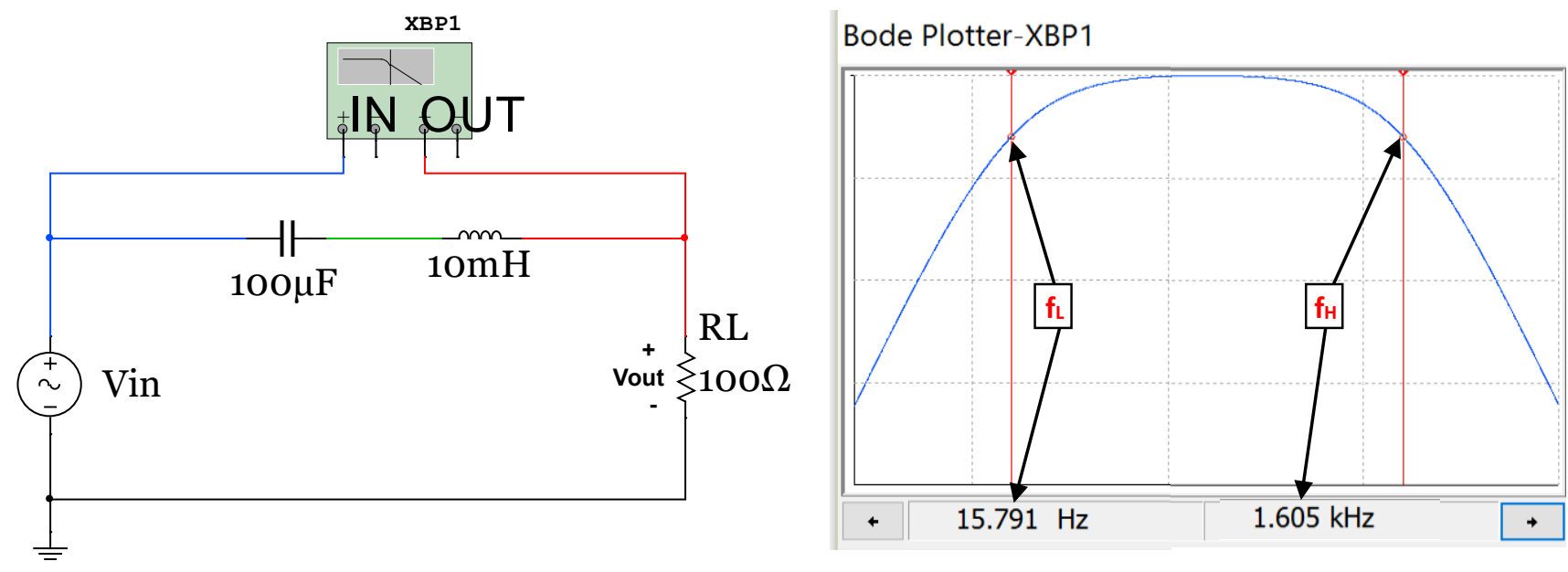

Figure 4.13: $2^{\text {nd }}$ Order Low-Q RLC Band Pass Filter. 
The Bode plot in Figure 4.13 has $5 \mathrm{~dB}$ per division scale on the $y$-axis and a logarithmic scale on the $\mathrm{x}$-axis that increases 1 decade (a factor of 10 in Hertz) every division. The $y$-axis values for $f_{L}$ and $f_{H}$ are at gain values as close to $-3 \mathrm{~dB}$ as possible with the best resolution of the Bode plot that was possible. The Bode plot shows two cursors on the Bode plot, but in Multisim only one cursor is available. The Bode plots in sections 4.3 and 4.4 with two $3 \mathrm{~dB}$ frequencies shown were created by splicing two different Multisim Bode magnitude plots together.

When the pass band of a BPF is wide it is called a broad band or Low-Q filter and when the pass band is narrow they it is called a narrow band or High-Q filter. The $Q$ in Low- $Q$ and High-Q comes from the "Quality Factor" parameter. $\mathbf{Q}$ is used for RLC Band Pass filters as a measure of frequency selectivity. If $\underline{Q}$ is low then the filter is not designed to select a specific frequency, but pass a range of frequencies instead. To determine the Quality Factor the resonant frequency needs to first be calculated using Equation 4.7. RLC circuits like those shown in Figures 4.13 and 4.14 tend to resonate (or cause oscillations) at a specific frequency as shown in Figure 4.15. This frequency is also called the "center frequency" because it is the geometric mean of the pass band as shown in Equation 4.8. The pass band region is quantified by a term called bandwidth (BW) that is the frequency range between the low $3 \mathrm{~dB}$ frequency $\left(\mathrm{f}_{\mathrm{L}}\right)$ and the high $3 \mathrm{~dB}$ frequency $\left(\mathrm{f}_{\mathrm{H}}\right)$ as shown in Equation 4.9.

$$
\text { Resonant Frequency }=f_{0}=\text { Center Frequency }=f_{c}=\frac{1}{2 \pi \sqrt{L \cdot C}}
$$

[4.8] $f_{C}=\sqrt{f_{L} \cdot f_{H}}$

[4.9] $B W=f_{H}-f_{L}$

[4.10] Quality Factor $=Q=\frac{f_{c}}{B W}$

Equations 4.7 through 4.10 are true for the series RLC BPF shown in this section regardless of the values of the components. For the circuit in Figure 4.13 the Equation 4.7 produces the following results:

$$
\text { - } f_{0}=f_{c}=\frac{1}{2 \pi \sqrt{L \cdot C}}=\frac{1}{2 \pi \sqrt{10^{-3} \mathrm{H} \cdot 100^{-6} \mathrm{~F}}}=159.155 \mathrm{~Hz}
$$

For a Low-Q Band Pass Filters with a large bandwidth (at least 2 decades of frequency difference between $f_{L}$ and $\mathrm{f}_{\mathrm{H}}$ like the BPF example shown in Figure 4.12) the low and high $3 \mathrm{~dB}$ frequencies can be approximated fairly closely with equations 4.11 and 4.12 , which are the same equations used for $1^{\text {st }}$ order LPF and HPF circuits.

[4.11] $f_{L} \cong \frac{1}{2 \pi \cdot R \cdot C} *$ This is accurate for series RLC BPF when $\mathrm{f}_{\mathrm{H}}$ and $\mathrm{f}_{\mathrm{L}}$ are separated by 2 decades.

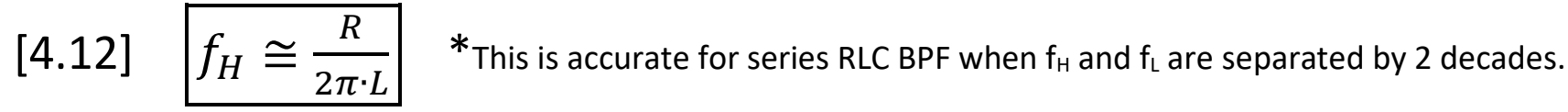

When $f_{L}$ and $f_{H}$ become closer together, Equation 4.11 and Equation 4.12 become less accurate. For example, in the circuit in Figure 4.13, Equation 4.11 and Equation 4.12 calculate the values as follows (The Multisim values are in Blue Font): 
- $f_{L} \cong \frac{1}{2 \pi \cdot R \cdot C}=\frac{1}{2 \pi \cdot 100 \Omega \cdot 100^{-6} \mathrm{~F}}=15.9 \mathrm{~Hz}$ Multisim: $15.791 \mathrm{~Hz}$

- $f_{H} \cong \frac{R}{2 \pi \cdot L}=\frac{100 \Omega}{2 \pi \cdot 10^{-3} H}=1.59 \mathrm{kHz} \quad$ Multisim: $1.605 \mathrm{kHz}$

In this example $f_{L}$ and $f_{H}$ obey the rule of thumb that states the $3 \mathrm{~dB}$ frequencies $\left(f_{L}\right.$ and $\left.f_{H}\right)$ need to be two decades or more apart for the approximations of equations 4.11 and 4.12 to be accurate. Using the approximate calculations in equations 4.11 and 4.12 the bandwidth and quality factor can be calculated as:

- $B W=f_{H}-f_{L}=1.59 \mathrm{kHz}-15.9 \mathrm{~Hz}=1575.6 \mathrm{~Hz}$

- $Q=\frac{f_{C}}{B W}=\frac{159.155 \mathrm{~Hz}}{1575.6 \mathrm{~Hz}}=0.1$

A series RLC BPF circuit with a $Q$ value of 0.1 can be safely called Low-Q. Furthermore, if the capacitor in the circuit in Figure 4.13 is changed to a value of $10 \mu \mathrm{F}$ then $\mathrm{f}_{\mathrm{L}}$ becomes $159 \mathrm{~Hz}$ which is only one decade from $\mathrm{f}_{\mathrm{H}}$. When this circuit (with a $10 \mu \mathrm{F}$ capacitor) is simulated in Multisim the $\mathrm{f}_{\mathrm{L}}$ and $\mathrm{f}_{\mathrm{H}}$ values are $\underline{147 \mathrm{~Hz}}$ and $\underline{1.734 \mathbf{~ k H z}}$ respectively, which shows equations 4.11 and 4.12 have significant error when the $3 \mathrm{~dB}$ frequencies are only one decade apart. Using the approximate calculations in equations 4.11 and 4.12 , the bandwidth and quality factor can be calculated as follows:

Note: The number in Blue Font is the BW determined from Multisim which will be much more accurate than equations 4.11 and 4.12 when $f_{L}$ and $f_{H}$ are only one decade apart.

- $f_{C}=\frac{1}{2 \pi \sqrt{L \cdot C}}=\frac{1}{2 \pi \sqrt{10^{-3} \mathrm{H} \cdot 10^{-6} \mathrm{~F}}}=503.3 \mathrm{~Hz}$

- $B W=f_{H}-f_{L}=1.59 \mathrm{kHz}-159 \mathrm{~Hz}=1432.4 \mathrm{~Hz} \quad$ Multisim: $\mathbf{B W}=\mathbf{1 5 8 7} \mathrm{Hz}$

- $Q=\frac{f_{C}}{B W}=\frac{503.3 \mathrm{~Hz}}{1432.4 \mathrm{~Hz}}=0.35$

A Q value of 0.35 would still probably be considered a Low- $Q$ filter, but there is no simple method or equation to use to easily calculate $f_{L}$ and $f_{H}$ for that circuit. Unfortunately, there is no perfect $Q$ cut-off point that separates a High- $Q$ from a Low- $Q$ filter, but there are approximate equations that can be accurately used for series RLC circuits that are extremely Low-Q or extremely High-Q. Equations 4.11 and 4.12 can be accurately used for extremely Low-Q series RLC circuits like shown in Figure 4.13 where $f_{H}$ and $f_{L}$ separated by 2 decades (Note:. The $Q$ value was 0.1 for the circuit in Figure 4.13).

Example 4.3) Design a circuit that filters out frequencies that are NOT in the audio range (20 Hz to $20 \mathrm{kHz}$ ). Use the Bode plot to determine the output due to the following two signals.

- $v_{\text {in1 }}(t)=5 \sin (2 \pi \cdot 1000 \cdot t)$ This signal should pass through the filter with little to no attenuation.

- $v_{\text {in2 }}(t)=2.3 \sin (2 \pi \cdot 50,000 \cdot t+0.2 \pi)$ This signal is beyond $20 \mathrm{kHz}$ so it should be significantly attenuated. A low-Q BPF can be used since the upper and lower frequency limits are at least 2 decades apart. If we set the $3 \mathrm{~dB}$ frequencies at $20 \mathrm{~Hz}\left(\mathrm{f}_{\mathrm{L}}\right)$ and $20 \mathrm{kHz}\left(\mathrm{f}_{\mathrm{H}}\right)$ only the edges of the audio range will be slightly attenuated. The resistor needed in the series RLC BPF circuit can be selected as many different values and still achieve the desired $3 \mathrm{~dB}$ frequencies. To keep the current in the circuit fairly low a resistor value of $\mathbf{1} \mathbf{k} \boldsymbol{\Omega}$ will be selected. Equations 4.11 and 4.12 are used to determine the values of the capacitor and inductor that is needed.

- Set $f_{L}=\frac{1}{2 \pi \cdot R \cdot C}=20 \rightarrow C=\frac{1}{2 \pi \cdot R \cdot f_{L}}=\frac{1}{2 \pi \cdot 1000 \cdot 20}=\mathbf{7 . 9 6} \mu \boldsymbol{F}$ 
- Set $f_{H}=\frac{R}{2 \pi \cdot L}=20,000 \rightarrow L=\frac{R}{2 \pi \cdot f_{H}}=\frac{1000}{2 \pi \cdot 20,000}=\mathbf{7 . 9 6} \boldsymbol{m H}$

The following shows the final circuit design along with the Bode magnitude plot showing $f_{L}$ is at $20 \mathrm{~Hz}$ and $f_{H}$ is at $20 \mathrm{kHz}$. Two separate Bode Magnitude plots are shown so the exact gain can be seen.
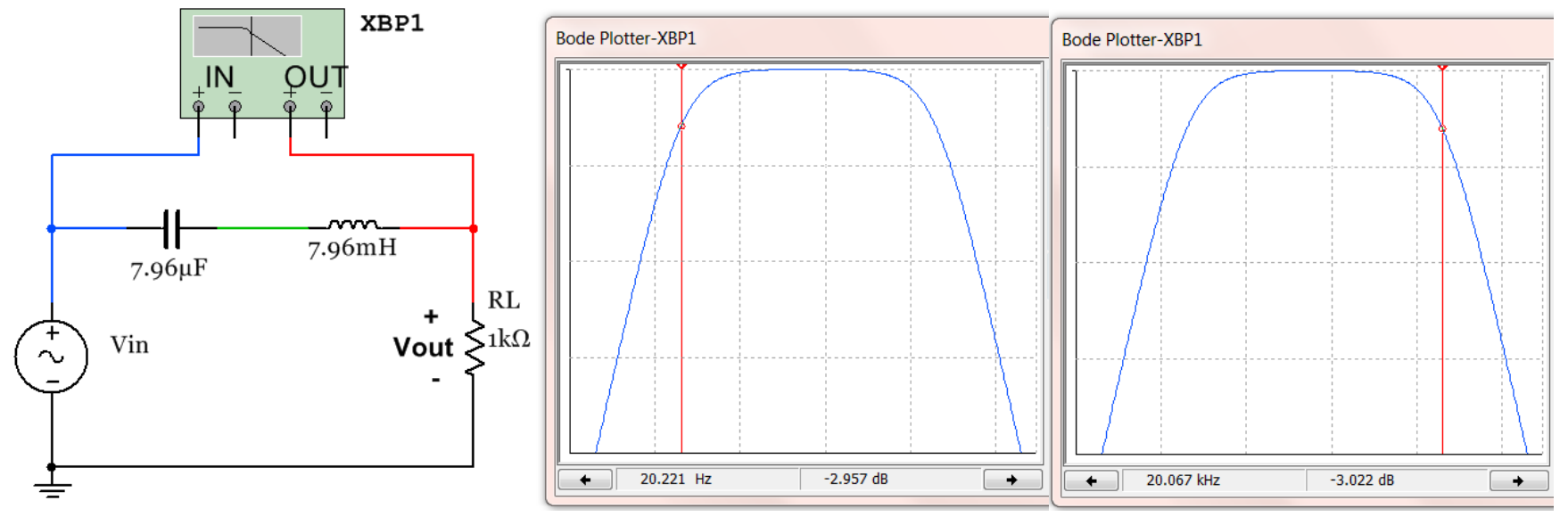

Next, the Bode Plot is shown at the frequency of $V_{\text {in } 1}(1 \mathrm{kHz})$ and $V_{\text {in2 }}(50 \mathrm{kHz})$.
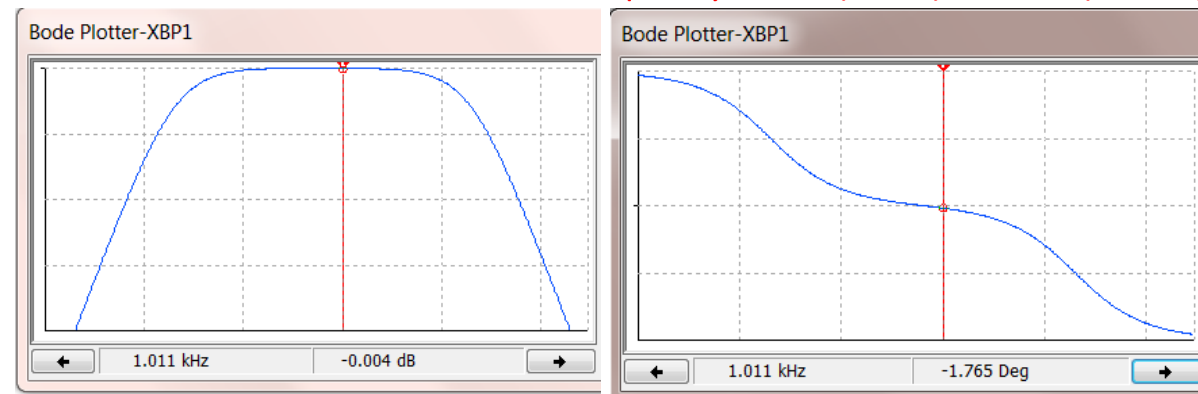

$$
\begin{aligned}
& V_{\text {in } 1}(t)=5 \sin (2 \pi \cdot 1000 \cdot t) \\
& \text { Gain }=-004 \mathrm{~dB} \rightarrow 0.99954 \mathrm{~V} / \mathrm{V} \\
& \Delta \text { Phase }=-1.7765^{\circ} \rightarrow-0.0099 \pi \\
& V_{\text {out } 1}(t)=5.0 .99954 \sin (2 \pi \cdot 1000 \cdot t-0.0099 \pi) \\
& V_{\text {out } 1}(t)=4.998 \sin (2 \pi \cdot 1000 \cdot t-0.0099 \pi)
\end{aligned}
$$
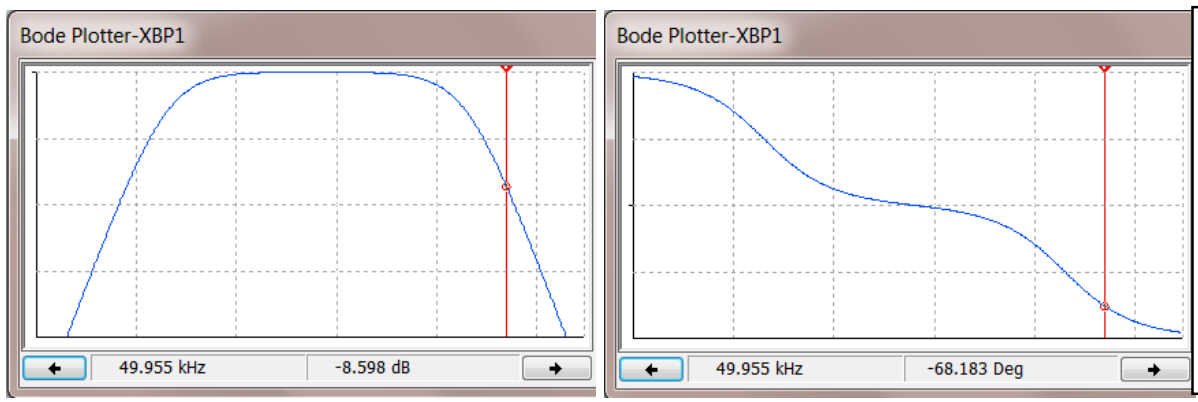

$v_{\text {in } 2}(t)=2.3 \sin (2 \pi \cdot 50000 \cdot t+0.2 \pi)$

Gain $=-8.598 d B \rightarrow 0.3716 \mathrm{~V} / \mathrm{V}$

$\Delta$ Phase $=-68.183^{\circ} \rightarrow-0.379 \pi$

$V_{\text {out } 2}(t)=2.3 \cdot 0.3716 \sin (\omega \cdot t+0.2 \pi-0.379 \pi)$

$V_{\text {out } 2}(t)=0.855 \sin (2 \pi \cdot 50000 \cdot t-0.179 \pi)$

Next, equations 4.13 and 4.16 are introduced that can be accurately used for extremely High-Q series RLC circuits. If the $Q$ value is calculated to be 10 or greater, like the one shown in Figure 4.14, great accuracy is achieved, but when $Q$ drops lower than 10 equations 4.13 to 4.16 become less accurate as shown in the following example for a series RLC BPF circuit with $Q=3.16$.

For High-Q filters $Q$ and BW can be calculated using equations $\underline{4.13}$ and $\underline{4.14}$ and then $f_{L}$ and $f_{H}$ can be calculated using equations $\underline{4.15}$ and $\underline{4.16}$. These equations produce very close approximations when $Q$ is 10 or greater as shown in the next example. Equation 4.13 shows that $X_{C}$ equals $X_{L}$ at the center frequency, $f_{C}$, for High-Q BPFs.

[4.13] $Q=\frac{X_{L}}{R}=\frac{2 \pi \cdot f_{C} \cdot L}{R}=\frac{X_{C}}{R}=\frac{1}{2 \pi \cdot f_{C} \cdot R \cdot C} \quad$ Only use Equation 4.13 if $Q \geq 10$. 

[4.14] $B W=\frac{f_{c}}{Q}$
[4.15] $f_{L} \cong f_{C}-\frac{B W}{2}$
[4.16] $f_{H} \cong f_{c}+\frac{B W}{2}$

When the capacitor in the series RLC circuit in Figure 4.13 is changed to a much smaller value, $f_{L}$ moves to the right (as shown in Figure 4.14), which results in the bandwidth narrowing and Q increasing. Now the High-Q filter equations (4.13 to 4.16) can be used to produce accurate results (see the Multisim comparison below).
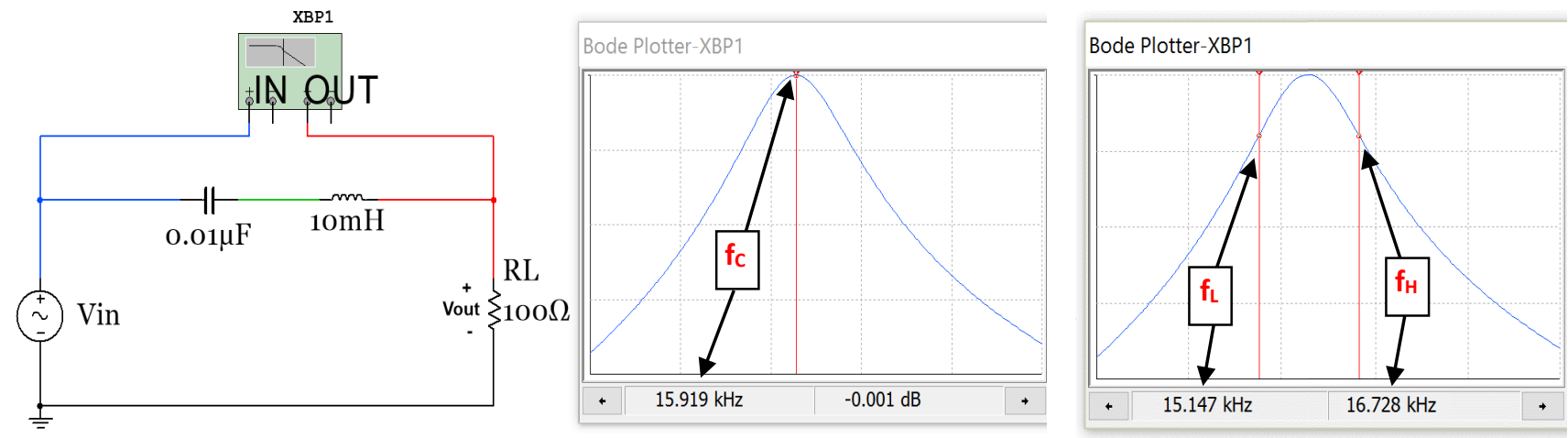

Figure 4.14: $2^{\text {nd }}$ order High-Q RLC Bandpass Filter with 2 screen shots of the Bode Magnitude plot.

- $f_{C}=\frac{1}{2 \pi \sqrt{L \cdot C}}=\frac{1}{2 \pi \sqrt{0.01 H \cdot 0.01^{-6} F}}=15.9155 \mathrm{kHz} \quad$ Multisim: $\mathbf{f}_{\mathrm{C}}=15.919 \mathrm{kHz}$

- $Q=\frac{X_{C}}{R}=\frac{X_{L}}{R}=\frac{2 \pi \cdot f_{c} \cdot L}{R}=\frac{2 \pi \cdot 15915.5 \mathrm{~Hz} \cdot 0.01 \mathrm{H}}{100 \Omega}=\underline{10}$

- $B W=\frac{f_{c}}{Q}=\frac{15.919 \mathrm{kHz}}{10}=1.59155 \mathrm{kHz}$

- $f_{L} \cong 15.9155 \mathrm{kHz}-\frac{1591.55 \mathrm{kHz}}{2}=15.12 \mathrm{kHz} \quad$ Multisim: $\mathbf{f}_{\mathrm{L}}=\mathbf{1 5 . 1 4 7} \mathrm{kHz}$

- $f_{H} \cong 15.9155 \mathrm{kHz}+\frac{1591.55 \mathrm{kHz}}{2}=16.71 \mathrm{kHz}$ Multisim: $\mathbf{f}_{\mathrm{H}}=\mathbf{1 6 . 7 2 8 ~ k H z}$

This shows when $Q$ equal to 10 equations 4.15 and 4.16 give very close approximations, but if the capacitor is increased to a value of $0.1 \mu \mathrm{F}$ and $Q$ is reduced the approximation is not as accurate (as shown below).

- $f_{C}=\frac{1}{2 \pi \sqrt{L \cdot C}}=\frac{1}{2 \pi \sqrt{0.01 H \cdot 0.1^{-6} F}}=5.033 \mathrm{kHz} \quad$ Multisim: $\mathbf{f}_{\mathrm{C}}=\mathbf{5 . 0 3 8 \mathrm { kHz }}$

- $Q=\frac{X_{C}}{R}=\frac{X_{L}}{R}=\frac{2 \pi \cdot f_{c} \cdot L}{R}=\frac{2 \pi \cdot 5.033 \mathrm{kHz} \cdot 0.01 \mathrm{H}}{100 \Omega}=\underline{\mathbf{3 . 1 6}}$

- $B W=\frac{f_{c}}{Q}=\frac{50.33 \mathrm{kHz}}{3.16}=1591.55 \mathrm{kHz}$

- $f_{L} \cong 50.33 \mathrm{kHz}-\frac{1591.55 \mathrm{kHz}}{2}=4.237 \mathrm{kHz} \quad \underline{\text { Multisim: } \mathbf{f}_{\mathrm{L}}=\mathbf{4 . 3 0 1} \mathbf{~ k H z}}$

- $f_{H} \cong 50.33 \mathrm{kHz}+\frac{1591.55 \mathrm{kHz}}{2}=5.829 \mathrm{kHz} \quad \underline{\text { Multisim: } \mathbf{f}_{\mathbf{H}}=\mathbf{5 . 8 9 4} \mathbf{~ k H z}}$ 
These values show that when $\mathrm{C}$ was increased to $0.1 \mu \mathrm{F}$ and $\underline{\mathbf{Q}=\mathbf{3 . 1 6}}$ the approximations of equations 4.15 and 4.16 are still fairly accurate, but not nearly as accurate as when $Q$ was equal to 10 for the circuit in Figure 4.14.

In summary, there is not a perfect cut-off for the Quality Factor, $Q$, which classifies a series RLC BPF as High-Q or Low-Q. The following Rules of Thumb help determine which equations to use to calculate $f_{L}$ and $f_{H}$.

- You should only expect "very close" results (i.e. that the calculated $f_{L}$ and $f_{H}$ frequencies are at $-3 d B$ gain points) with Equations 4.15 and 4.16 when $\underline{Q \geq 10}$. $Q$ values of 10 or greater should definitely classify the series BPF as High-Q.

- You should only expect "very close" results with Equations 4.11 and 4.12 for $f_{L}$ and $f_{H}$ when $\underline{f}_{L}$ and $f_{H}$ are $\mathbf{2}$ decades in frequency apart. If this occurs the BPF or BSF should definitely be classified as Low-Q.

- If $\underline{f}_{L}$ and $f_{H}$ are closer to each other than 2 decades in frequency and the $\underline{Q}$ value is less than 10 then there is no easy way to accurately determine the values of $f_{L}$ and $f_{H}$. This would be a problem that would need to be solved using the Laplace Transform method (as shown in the Appendix). In this situation, the determination of whether to classify the filter as High-Q or Low-Q is also not clear.

Before moving on to Band Stop Filters the idea of resonance in RLC circuits and the "resonant frequency" defined in Equation 4.7 should be explained in more detail. For the circuit in Figure 4.14 the resonant frequency, $f_{0}$, (or center frequency, $f_{c}$ ) is calculated as $15.92 \mathrm{kHz}$. The figure below shows the transient response of the circuit in Figure 4.14 when the input voltage is a $1 \mathrm{~V} \mathrm{DC}$ signal that is suddenly applied (i.e. a step input, $\mathrm{u}(\mathrm{t})$ ). Since the capacitor acts as an open circuit at DC the voltage across the resistor will approach zero at steady state. However, in the transient region the voltage across the resistor will oscillate (or resonate) at the resonant frequency until the oscillations die out as steady state is reached as shown in Figure 4.15. The cursors in Figure $\underline{4.15}$ are placed at locations where the difference between them is the half-period of the oscillations. $T / 2$ can be determined from these cursors as $(0.000518-0.000487=0.000031$ seconds $)$ which equates to a frequency of $(1 /(2 \cdot 0.000031)=\sim 15.92 \mathrm{kHz})$ which is equal to the calculated resonant frequency from Equation 4.7. The Matlab bode magnitude plot in Figure 4.15 is also shown to match the Multisim Bode plot in Figure 4.14 closely. 


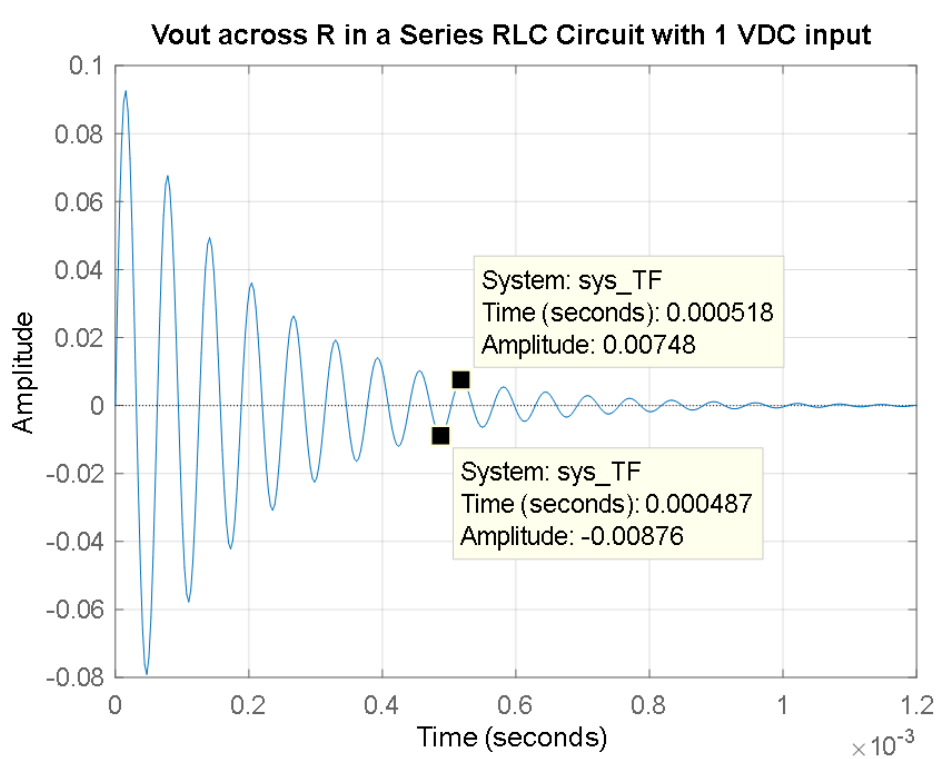

System: sys_TF

Frequency $(\mathrm{Hz}): 1.59 \mathrm{e}+04$

Bode Diagram

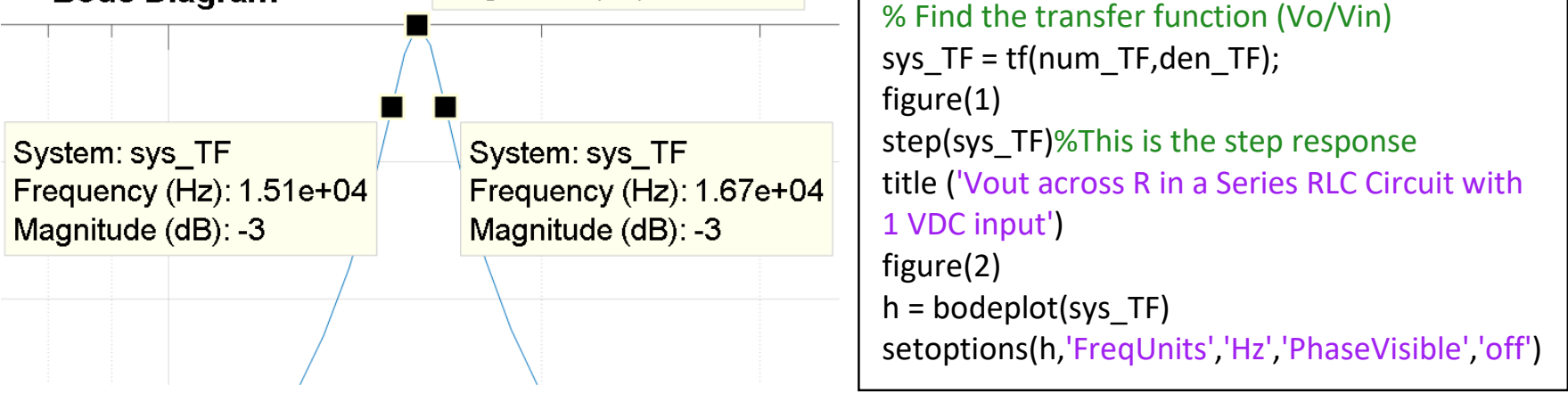

Figure 4.15: Matlab step response and Bode plot simulation of the $2^{\text {nd }}$ order High-Q filter circuit in Figure 4.14.

\section{Section 4.4 -Band Stop Filters (BSF)}

Band Stop Filter (BSF) will not be covered in as much detail as the other filters since its applications are not as numerous as the others. A Band Stop Filter (also called Band Reject Filter or Notch Filter) is essentially the opposite of a BPF because it partially rejects a range of frequencies in its stop band, but passes low and high frequencies that are outside the stop band. The term notch filter is used in the previous sentence because it is a much more descriptive term than BSF. The notch in the filter can be used to eliminate specific noise that is present in a circuit. A common application of a notch filter is to remove $60 \mathrm{~Hz}$ noise from AC devices that are plugged into AC wall outlets. Wires or cables that travel in close proximity to $60 \mathrm{~Hz}$ noise sources tend to pick up noise, which can sometimes negatively affect the performance of the circuit. By adding in a $60 \mathrm{~Hz}$ notch filter the noise can be eliminated to improve the performance of the circuit.

A common $2^{\text {nd }}$ order ( 2 reactive elements: 1 inductor and 1 capacitor) BSF circuit is shown in Figures 4.16 . The Bode magnitude plot and calculations of the center frequency $\left(f_{c}\right)$ and the bandwidth $(B W)$ are also included. These two parameters characterize the notch in the BSF and the equations that apply to them are shown in 
equations 4.7 and 4.9. The steeper the notch the more "selective" the filter is at rejecting frequencies. The term quality factor $(Q)$ shown in Equation 4.10 is used to quantify how selective the notch is. As described in Section $\underline{4.3}$, the quality factor was also used to determine how selective a BPF is at "passing" the center frequency. The $2^{\text {nd }}$ order Low-Q BPF in Figure 4.13 is in many ways the opposite of the BSF shown in Figure 4.16. The parallel capacitor and inductor result in something frequently referred to as a "Tank Circuit", which oscillates at its resonant frequency and stores energy (like a storage tank). Notice that the inductor causes $f_{L}$ and the capacitor causes $f_{H}$, but it was the opposite for the series RLC BPF. The center frequency of the tank-style RLC BSF circuit also uses Equation 4.7 like the series RLC BPF.

Note: The quality factor (and High-Q and Low-Q classifications) will not be applied to the Band Stop Filter and only the following two equations will be given for the situation when $f_{H}$ and $f_{L}$ are separated by 2 decades.

[4.17] $f_{L} \cong \frac{R}{2 \pi \cdot L} *$ This is accurate for tank-style RLC BSF when $\mathrm{f}_{\mathrm{H}}$ and $\mathrm{f}_{\mathrm{L}}$ are separated by 2 decades.

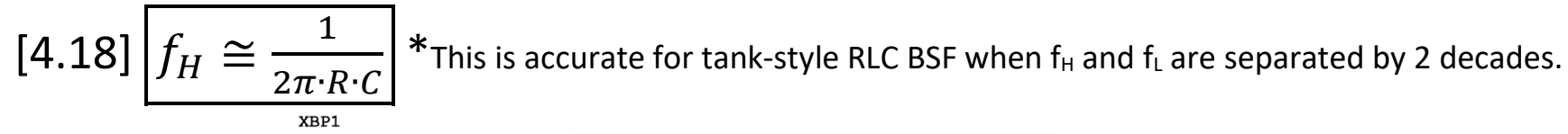
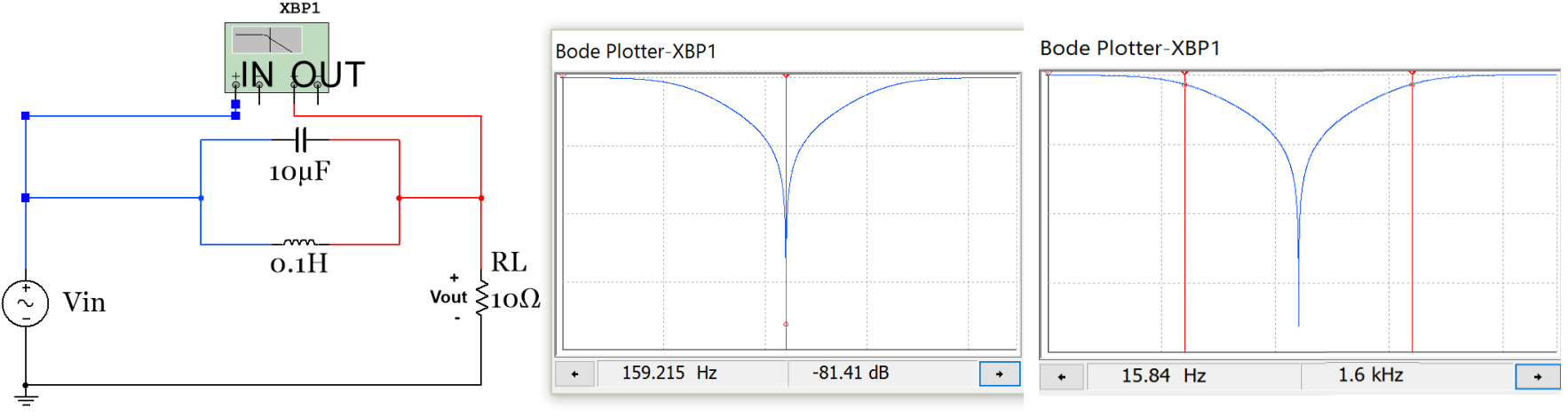

Figure 4.16: $2^{\text {nd }}$ order RLC Band Stop Filter.

This BSF circuit in Figure 4.16 (with $\underline{L}=100 \mathrm{mH}$ ) has the same $f_{C}, f_{L}$, and $f_{H}$ as the BPF circuit in Figure 4.13 (as shown in the bulleted calculations below). The only difference (other than it is the opposite type of filter with the pass band and stop bands reversed) is that $f_{H}$ comes from the capacitor and $f_{L}$ comes from the inductor.

- $f_{C}=\frac{1}{2 \pi \sqrt{L \cdot C}}=\frac{1}{2 \pi \sqrt{0.1 H \cdot 10^{-6} F}}=159.155 \mathrm{~Hz}$ Multisim: $159.215 \mathrm{~Hz}$

- $f_{H} \cong \frac{1}{2 \pi \cdot R \cdot C}=\frac{1}{2 \pi \cdot 10 \Omega \cdot 10^{-6} F}=1.59 \mathrm{kHz}$ Multisim: $1.6 \mathrm{kHz}$

- $f_{L} \cong \frac{R}{2 \pi \cdot L}=\frac{10 \Omega}{2 \pi \cdot 0.1 \mathrm{H}}=15.9 \mathrm{~Hz}$ Multisim: $15.84 \mathrm{~Hz}$

- $B W=f_{H}-f_{L}=1.59 \mathrm{kHz}-15.9 \mathrm{~Hz}=1575.6 \mathrm{~Hz}$ Multisim: $\mathbf{B W}=\mathbf{1 5 8 4} \mathrm{Hz}$

If the inductor in Figure 4.16 is changed to a value of $\underline{L=10 \mathrm{mH}}$ then $\mathrm{f}_{\mathrm{L}}$ becomes $\underline{159 \mathrm{~Hz}}$ which is only one decade from $f_{H}$. When this circuit (with a $10 \mathrm{mH}$ inductor) is simulated in Multisim the $f_{\mathrm{L}}$ and $\mathbf{f}_{\mathrm{H}}$ values are $146.3 \mathrm{~Hz}$ and $1.732 \mathrm{kHz}$ respectively and the bandwidth is $(1732-146.3=1585.7 \mathrm{~Hz})$. The Multisim simulated values are shown below to be much different than the calculated values from equations 4.17 and 4.18. The error results by using equations 4.17 and 4.18 when $f_{L}$ and $f_{H}$ are only being one decade apart.

- $f_{C}=\frac{1}{2 \pi \sqrt{L \cdot C}}=\frac{1}{2 \pi \sqrt{10^{-3} H \cdot 10^{-6} F}}=503.3 \mathrm{~Hz} \quad$ Multisim: $\mathrm{f}_{\mathrm{C}}=\mathbf{5 0 3 . 4 \mathrm { Hz }}$

- $B W=f_{H}-f_{L}=1.59 \mathrm{kHz}-159 \mathrm{~Hz}=1432.4 \mathrm{~Hz} \quad$ Multisim: $\mathbf{B W}=\mathbf{1 5 8 5 . 7} \mathrm{Hz}$ 


\section{Module 5 - Transformers}

A brief overview of transformers is needed in this eBook for two reasons. First of all, the invention of the transformer led to AC voltages being used as the primary electric power source worldwide (Read:

https://en.wikipedia.org/wiki/War of Currents for more information on this topic). Additionally, transformers are included in this eBook because without them rectifier circuits (Section 6.3) that convert an AC voltage to a DC voltage could not be adequately be covered. If this module is not in depth enough, the following two links from allaboutcircuits.com are recommended as additional resources.

http://www.allaboutcircuits.com/textbook/alternating-current/chpt-9/mutual-inductance-and-basic-operation/

\section{http://www.allaboutcircuits.com/video-lectures/transformers-part-1/}

Figure 5.1 shows a transformer with the following quantities labeled: primary voltage $\left(V_{P}\right)$, primary current $\left(I_{P}\right)$, secondary voltage $\left(V_{S}\right)$, secondary current $\left(I_{S}\right)$, and the turns ratio $\left(N_{p}: N_{S}\right.$ or $\left.N_{p} / N_{S}\right)$. The primary side of the transformer connects to the input and the secondary side of the transformer connects to the output (or load).

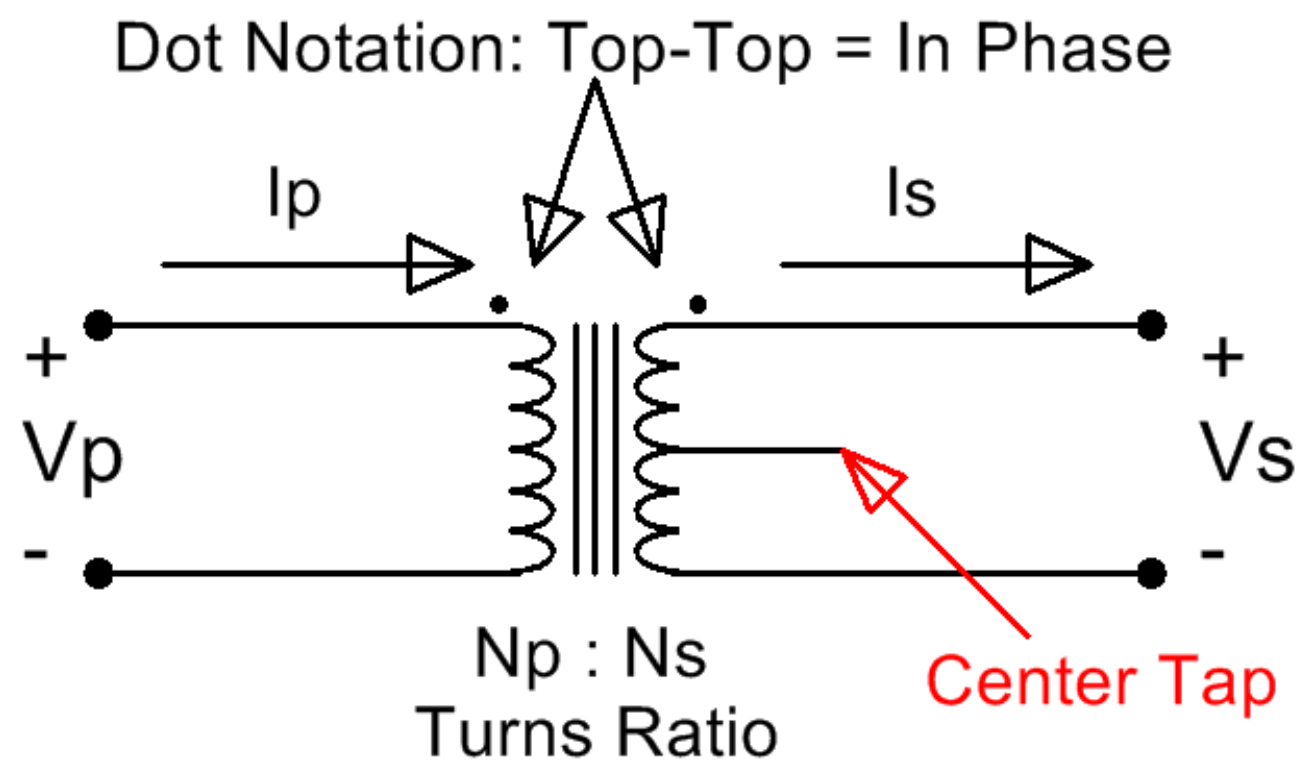

Figure 5.1: Iron Core Transformer Symbol

The vertical lines in the center of the transformer signify that it has an iron core. An iron core transformer is usually used for low frequency applications, including power transmission, which is the primary application addressed in this eBook. The curves on either side of the core represent the magnetic coil of wire that is wrapped around the primary and secondary sides of the transformer. The turns-ratio is the ratio of the number of primary coil turns of wire to the number of secondary coil turns of wire. The turns ratio is usually listed as $N_{p}: N_{s}$ or $N_{p} / N_{s}$. The middle line extending horizontally from the center of the secondary is called the center tap. Some transformers have this physical connection (or tap) that separates two sections of the secondary coil. If the tap is left unwired as shown in Figure 5.1 then the transformer will act as a standard transformer and can be used for half wave or full wave bridge rectifier circuits as shown in Section 6.3. However, if the middle wire is used then both sides of the tap will have its own turns-ratio value that must be factored into the problem. There are three possible wiring methods for standard transformer applications that use only two wires: top and middle legs, bottom and middle legs, and top and bottom legs. All three wires can be used in certain applications such as parallel (or center tap) full wave rectifier circuits (Shown in the third figure from the top in this link). The two 
dots above the primary and secondary coil symbols are used to label the type of phase change the transformer will have. The dot notation states that if both dots are on top (or if there is only a single dot on top) then the primary and secondary voltages will be in phase $\left(0^{\circ}\right.$ phase shift) and if one is on top and the other is on bottom then there will be a $180^{\circ}$ phase shift.

Governed by Faraday's law (Equation 5.1), current from the input voltage source $(\mathrm{Vp})$ is forced through the primary coil of wire, which results in a magnetic flux being generated in the iron core. The flux travels through the iron core and induces a voltage difference on the secondary coil of wire (Vs) as shown in Figure 5.2. $e=N \frac{d \Phi}{d t} *$ Where $\mathrm{e}$ is the induced voltage, $\mathrm{N}$ is the \# of turns, and $\Phi$ is the flux.

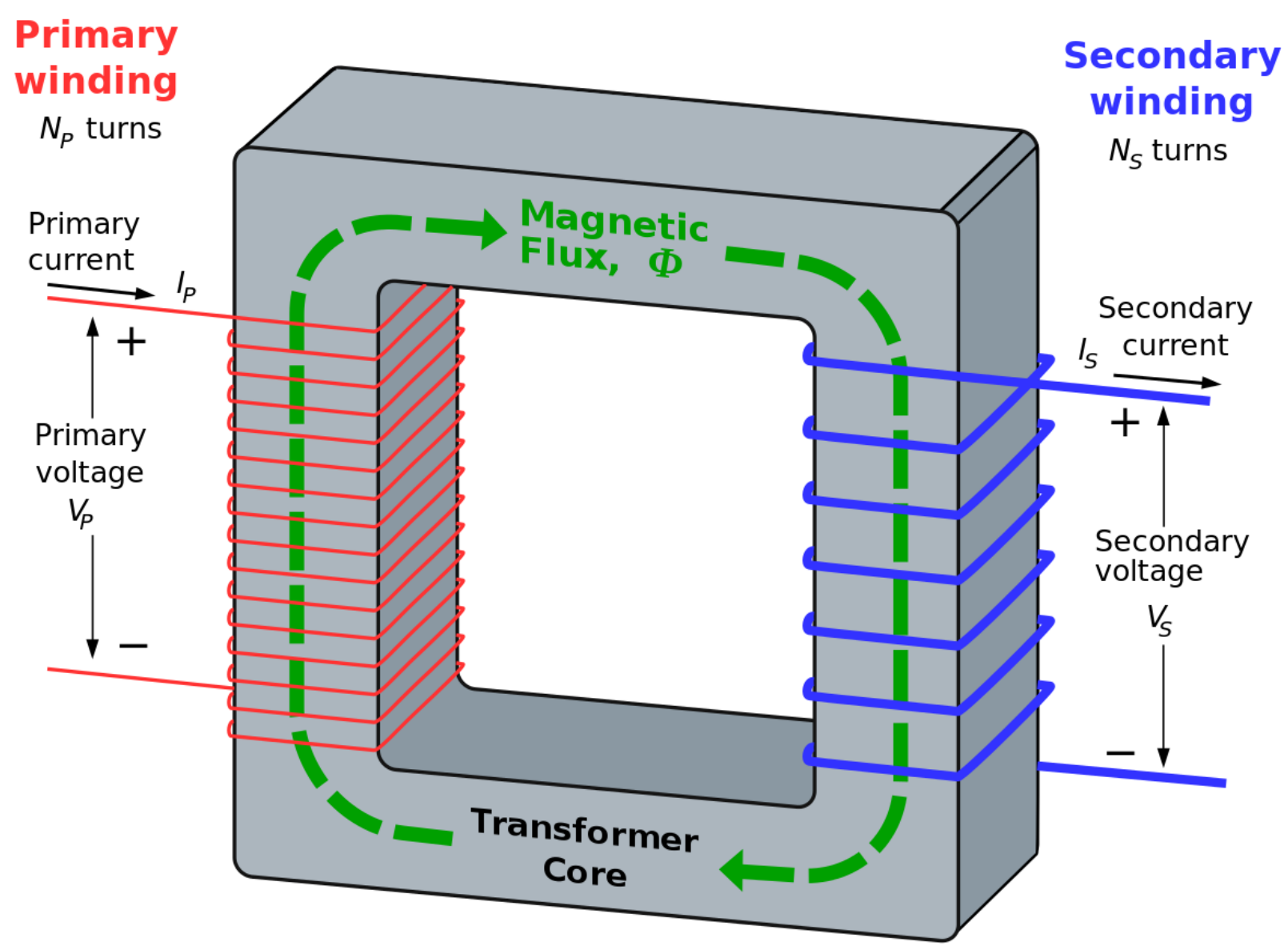

Figure 5.2: Internal Diagram of a Transformer. "Ideal transformer and induction law" image (C) BillC. Used under a CC BY-SA 3.0 license. 
In this eBook the transformer will be assumed to be ideal (100\% efficient), but there is magnetizing and leakage inductance as well as resistance in the primary and secondary coils that reduce the efficiency. If the standard (non-center tapped) transformer Multisim part called 1P1S (which stands for 1 primary and 1 secondary) is used the turns ratio as well as the inductances and resistances that reduce the efficiency can be entered. The default values for the 1P1S transformer are shown in Figure 5.3. The first tab allows the turns-ratio to be set. The next three tabs allow the imperfections that cause efficiency reduction to be set. For this part it allows each of these imperfections to be removed making the transformer truly idea. These settings will be set this way for the circuits in this eBook so that the calculations will match Multisim closely.

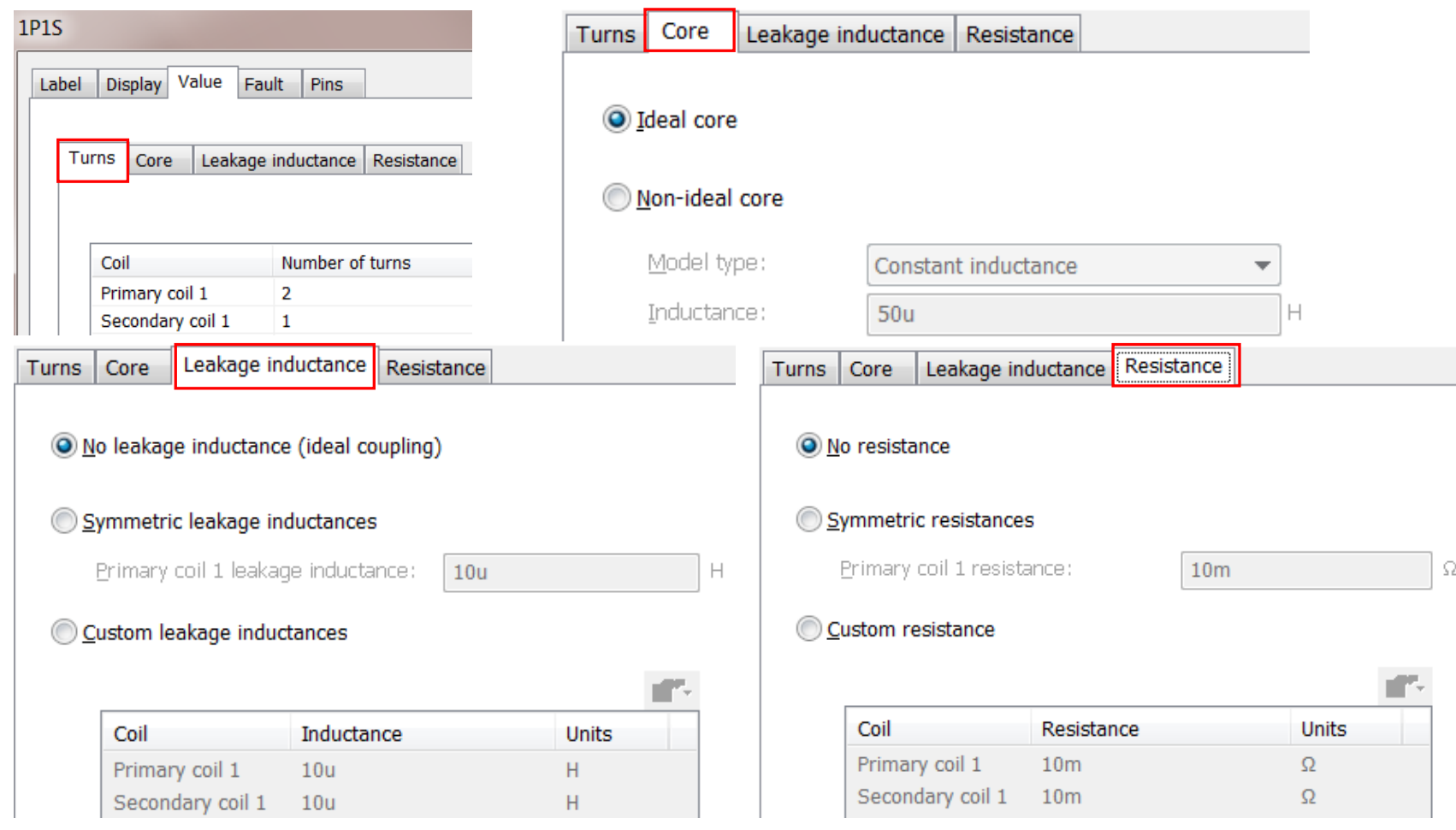

Figure 5.3: 1P1S Mustisim Transformer User Interface with resistance and inductance values set to ideal.

The ideal transformer equations that determine the ratios of the primary and secondary voltage and currents are shown in equations 5.2 and 5.3.

$[5.2] \frac{V_{P}}{V_{S}}=\frac{N_{P}}{N_{S}}$

$[5.3] \quad \frac{I_{S}}{I_{P}}=\frac{N_{P}}{N_{S}}$ 
Example 5.1) Calculate $V_{s}, I_{s}$, and $I_{P}$ for a 1S1P 2:1 ideal Step-Down transformer with a standard 120 Vrms wall outlet power signal connected to the primary and a $1 \mathrm{k} \Omega$ resistor connected to the secondary as shown in Figure 5.4. Also, calculate the primary and secondary power.

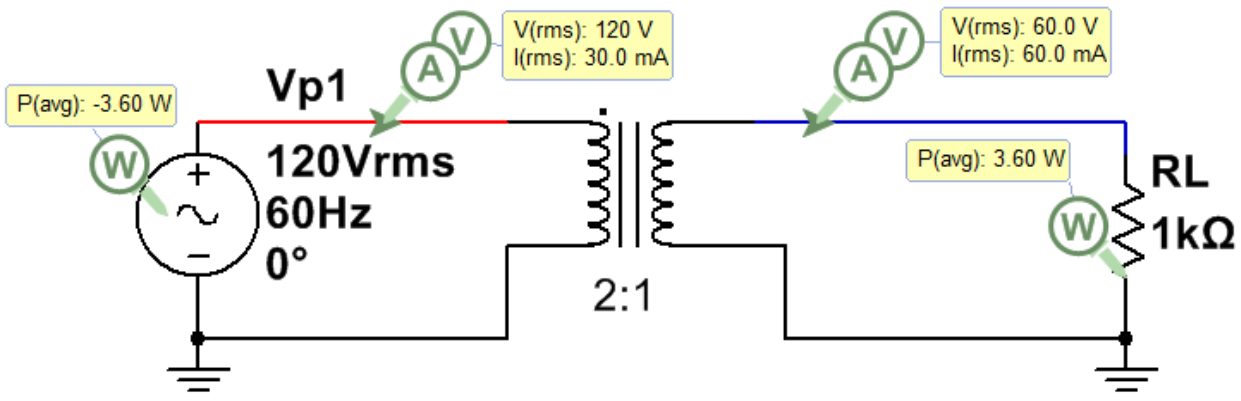

Figure 5.4: 2:1 Step Down Transformer for Example 5.1.

This problem must be solved in the correct sequence (as follows):

1) The secondary voltage can be calculated from Equation $5.2 \rightarrow V_{S}=V_{P} /\left(N_{P} / N_{S}\right)=120 / 2=\underline{60 ~ V r m s}$

2) The secondary current can be calculated using Ohm's Law $\rightarrow \mathrm{I}_{\mathrm{S}}=\mathrm{V}_{\mathrm{S}} / \mathrm{RL}=60 \mathrm{Vrms} / 1 \mathrm{k} \Omega=\underline{60 \mathrm{mArms}}$

3) The primary current can be calculated using Equation $5.3 \rightarrow \mathrm{I}_{\mathrm{p}}=\mathrm{I}_{\mathrm{s}} /\left(\mathrm{N}_{\mathrm{P}} / \mathrm{N}_{\mathrm{s}}\right)=60 \mathrm{mArms} / 2=\underline{\mathbf{3 0} \mathrm{mArms}}$

4) $100 \%$ efficient so $P_{P}=P_{S}=V_{P} \cdot I_{P}=V_{S} \cdot I_{S}=\underline{3.6 \mathrm{~W}}$

Keep in mind that a real transformer will NOT be $100 \%$ efficient. The voltage ratios in Equation 5.2 will remain close to true for non-ideal transformers, but the current ratio (Equation 5.3) will become inaccurate because additional primary current will be required by the source due to the power loss in the transformer.

The transformer in Example 5.1 is called a Step Down transformer because the voltage is reduced at the primary. A Step Down transformer has a turns-ratio > 1. Example 5.2 shows a Step Up transformer (turns-ratio < 1).

Example 5.2) Calculate $V_{s}, I_{s}$, and $I_{P}$ for a 1S1P 2:1 ideal Step-Down transformer with a standard 120 Vrms wall outlet power signal connected to the primary and a $1 \mathrm{k} \Omega$ resistor connected to the secondary. Also, calculate the primary and secondary power.

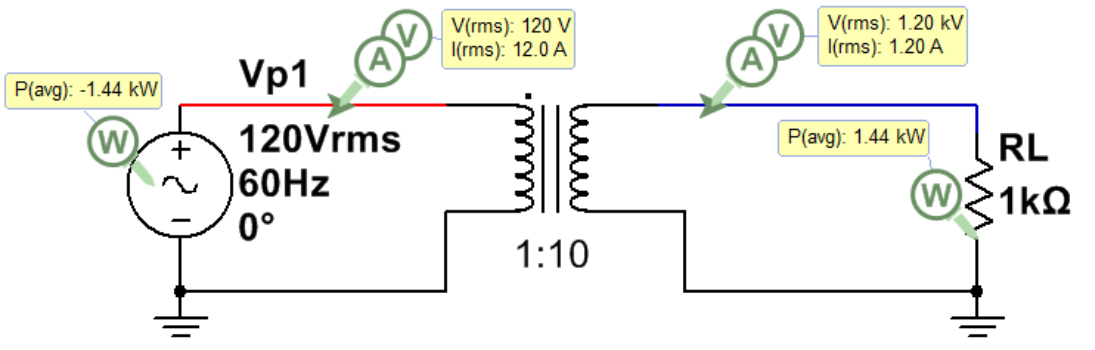

Transformer Calclations

1) $V s=V p /(N p / N s)=120 /(0.1)=1.2 \mathrm{kVrms}$

2) Is $=V s / R L=1.2 \mathrm{kVrms} / 1 \mathrm{k} \Omega=1.2 \mathrm{Arms}$

3) $\mathrm{Ip}=\mathrm{Is} /(\mathrm{Np} / \mathrm{Ns})=1.2 \mathrm{Arms} /(0.1)=12 \mathrm{Arms}$

4) $\mathrm{Pp}=\mathrm{Ps}=\mathrm{Vp} \cdot \mathrm{Ip}=\mathrm{Vs} \cdot \mathrm{Is}=1.44 \mathrm{~kW}$

Figure 5.5: 1:10 Step Up Transformer for Example 5.2. 


\section{Module 6- Diodes and AC to DC Conversion}

Note: There some additonal Diode example problems at the end of the Appendix. A diode is a device that acts like a current valve, where current is allowed to flow from anode to cathode, but not allowed to flow from cathode to anode (base of triangle). When current is allowed to flow the diode is said to be forward biased and when current is blocked it is reverse biased. There are many different types of diodes. The two main types discussed in this eBook are shown in Figures 6.1 (switching diodes) and 6.2 (rectifier diodes).

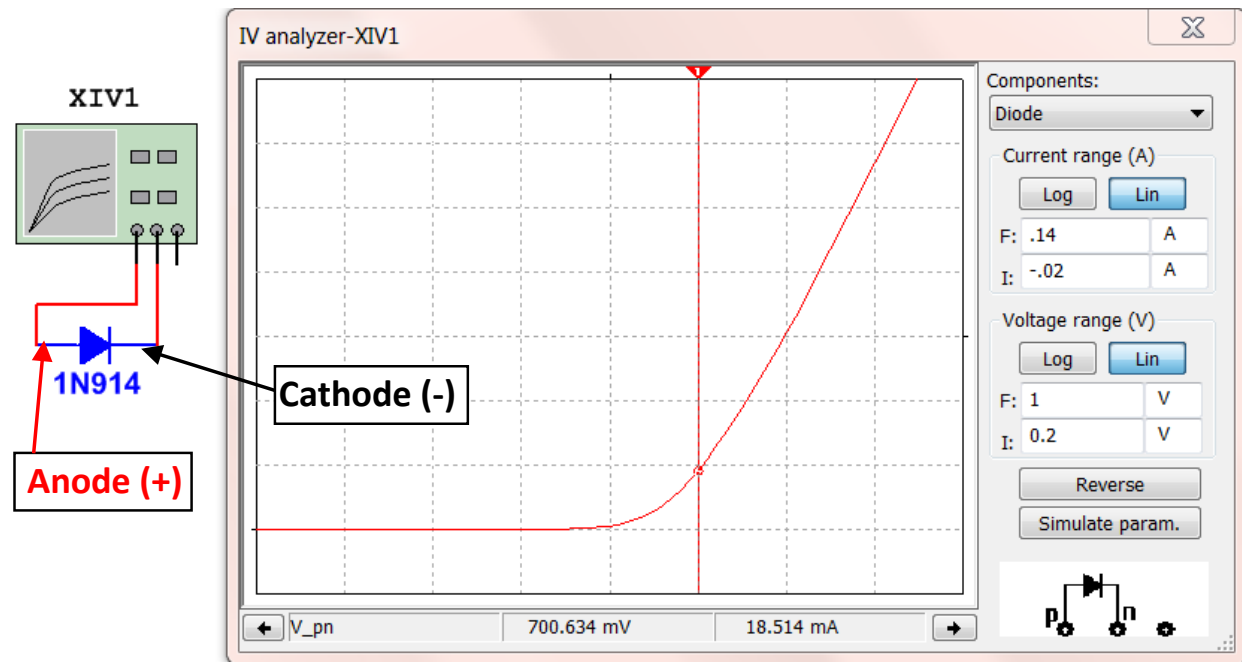

Silicon switching or signal diodes (Examples: $1 \mathrm{~N} 914$, 1N4148). This type of diode can switch between forward and reverse bias quickly. They are used for lowpower, high-speed switching applications. The voltage drop $\left(V_{D}\right)$ when forward biased is $\sim 0.7 \mathrm{~V}$, but depends on current as seen in the $1 \mathrm{~N} 914 \mathrm{I}-\mathrm{V}$ curve.

Figure 6.1: Characteristic Curve ( $I_{D}$ vs $V_{D}$ ) of a $1 \mathrm{~N} 914$ silicone switching diode (also called signal diode).

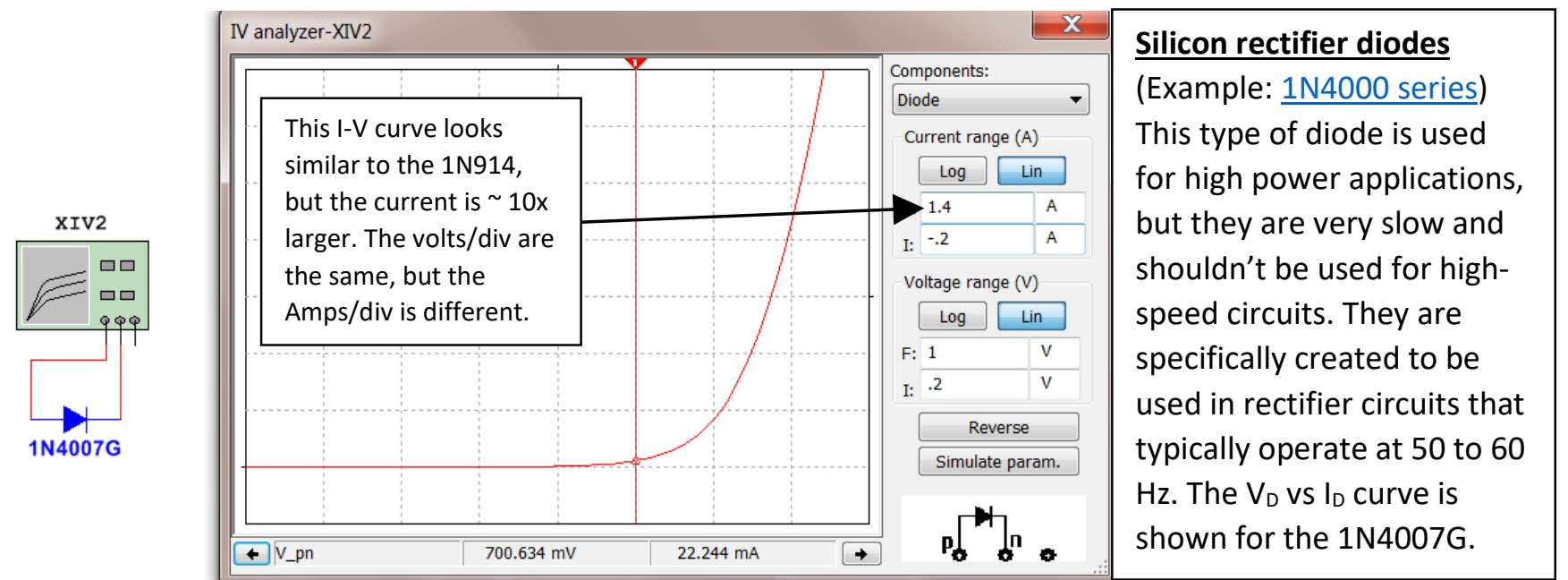

Figure 6.2: Characteristic Curve ( $I_{D}$ vs $\left.V_{D}\right)$ of a 1 N4007 silicone rectifier diode.

A few other types of diodes are discussed below. An $I_{D}$ vs. $V_{D}$ curve can be done for any diode using the Multisim I-V Analysis tool (as shown in Figures 6.1 and 6.2) if the voltage at a specific current is needed to be known.

- A Schottky diode can be used for rectifiers or switching circuits. They have a forward voltage drop much smaller than silicone diodes $\left(V_{D}=\sim 0.3 \mathrm{~V}\right)$.

- A Zener diode works differently than the other diodes discussed thus far. They are designed to work in the reverse voltage region (where the other diodes prevent current from flowing). They are often used for reference voltage or low-power voltage regulator applications.

- A Light emitting diode (LED) is a type of diode that emits light as current flows through it. The smaller LEDs that are often used in a breadboard typically have a current limit (or rating) of around $20 \mathrm{~mA}$. This 
Sparkfun tutorial is a good source for more information. Figure 6.3 shows the $I_{D} V_{S} . V_{D}$ curve for a typical low power Red LEDs (Left) and Green LEDs (Right). When too much current flows through a green LED the light color will change from green to yellow. This typically happens shortly before it is destroyed so if you see a green LED turn yellow cut off the power ASAP!

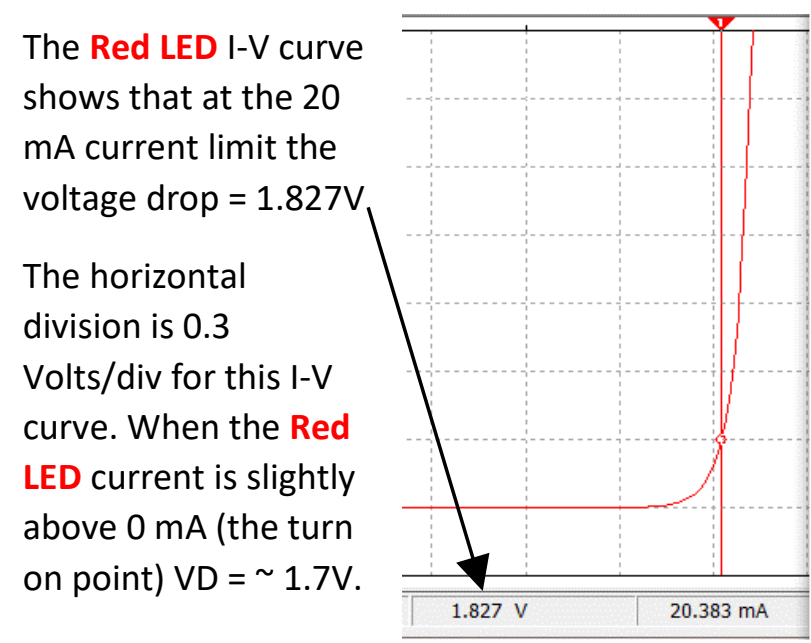

The Green LED I-V curve shows that at the $20 \mathrm{~mA}$ current limit the voltage drop $=2.131 \mathrm{~V}$ The horizontal division is 0.3 Volts/div for this I-V curve. When the Green LED current is slightly above $0 \mathrm{~mA}$ (the turn on point) $\mathrm{VD}=\sim 1.9 \mathrm{~V}$.

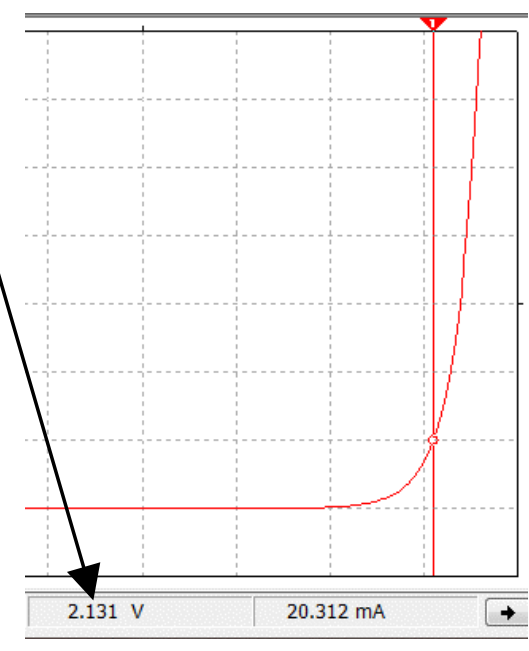

Figure 6.3: Characteristic Curve ( $\mathrm{D}_{\mathrm{D}} \mathrm{Vs} \mathrm{V}_{\mathrm{D}}$ ) of a low power $5 \mathrm{~mm}$ Red LED (Left) and Green LED (Right).

The following are some good links to learn more about diodes if the next three sections leave some questions unanswered: http://www.futureelectronics.com/en/diodes/diodes.aspx

http://www.allaboutcircuits.com/textbook/semiconductors/chpt-3/introduction-to-diodes-and-rectifiers/

\section{Section 6.1 - Diode Constant Drop Model}

In order to solve circuits that include diodes, a model for the diode is needed. There are many different types of models that can be used, but the one that offers the best combination of accurate results and ease of use is the Constant Drop Model. Before showing how to implement the Constant Drop Model, the process to solve problems with diodes in them is listed below. All of the circuits in this section will only include DC sources. By understanding how diodes affect circuits with DC sources, it is not a difficult jump to apply it to AC circuits also.

\section{Process for Solving DC Circuits with Diodes using the Constant Drop Model}

1) Assume Forward or Reverse Bias for each diode in the circuit.

2) Replace each diode with their model and solve for the forward bias current or reverse bias voltage.

a. Reverse Bias - Diode replaced open circuit. Solve for voltage drop from anode to cathode.

b. Forward Bias - Diode replaced with battery. Solve for current flowing from anode to cathode.

- The battery is placed with the + side where the anode of the diode was in the circuit. The value of the voltage is approximated based on the type of diode. Assume the following: Shottky Diode = $0.3 \mathrm{~V}$, Silicone switching or rectifier diodes $=0.7 \mathrm{~V}$, Red LED $=1.8 \mathrm{~V}$, Green LED $=2.1 \mathrm{~V}$.

3) Check the conditions for forward or reverse bias to make sure assumption in part 1 was correct:

a. Forward Bias Condition: $I_{D}>0$ (i.e. The current calculated in part 2 is flowing from anode to cathode)

b. Reverse Bias Condition: $V_{D}<$ Diode Turn On Voltage (i.e. The voltage calculated in part 2 is less than the values shown above $\rightarrow$ Schottky $=0.3 \mathrm{~V}$, Silicone $=0.7 \mathrm{~V}$, Red LED $=1.8 \mathrm{~V}$, Green LED $=2.1 \mathrm{~V}$.

4) If the assumptions from part 1 are verified in part 3 the problem is done. If any of the diodes don't meet the conditions in part 3, start the problem over by going back to step 1 and make different assumptions. 
Example 6.1) Follow the DC Diode Analysis process to solve the following circuit if $D 1$ is a silicone diode.

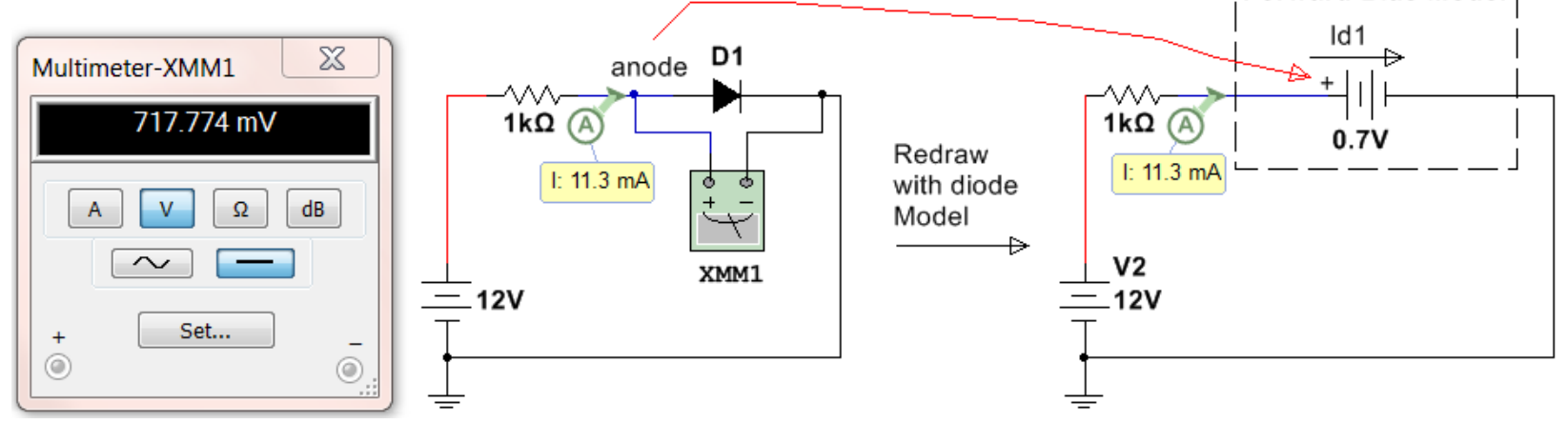

Figure 6.4: Circuit for Example 6.1

Step 1) D1 will be assumed to be forward biased because the $12 \mathrm{~V}$ source will force current clockwise in this circuit, which is the direction from the anode to cathode of the diode.

Step 2) The circuit on the right side of Figure 6.4 shows the forward bias model replacing the diode. The node where the anode was connected is connected to the + side of the battery. $0.7 \mathrm{~V}$ is used for the battery voltage because the problem stated it is a silicone diode. Next, calculate the current, $\mathrm{I}_{\mathrm{D} 1}$.

- $I_{D 1}=I_{R}=\Delta V / R=(12-0.7) / 1 \mathrm{k} \Omega=\underline{11.3} \mathbf{m A}$ (This is verified in Mulisim in Figure 6.4)

- Note: The actual diode voltage is shown in Multimeter XMM1 to be $717.774 \mathrm{mV}$, but there is no way to know the exact voltage across the diode unless a simulation tool is available. The approximate Constant Drop Model voltage used for silicone diodes is $0.7 \mathrm{~V}$ is very close to $0.717774 \mathrm{~V}$ so the calculations were identical to the Multisim calculation (within the accuracy of the current probe).

Step 3) Verify the diode is forward biased by making sure that $I_{D 1}>0$ and flowing from anode to cathode. The current is $11.3 \mathrm{~mA}$ and the direction is clockwise in the circuit (which is from the anode to cathode).

Step 4) The forward bias condition assumed in Step 1) is verified in Step 3) so the problem is done.

Example 6.2) Follow the DC Diode Analysis process to solve the following circuit if D2 is a silicone diode.
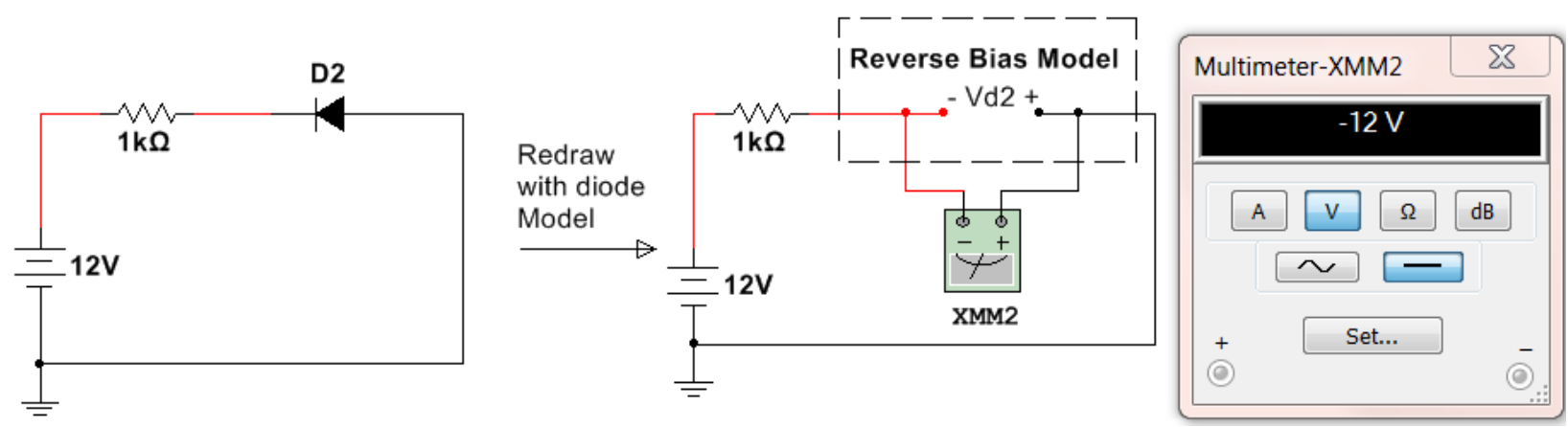

Figure 6.5: Circuit for Example 6.2

Step 1) D2 will be assumed to be reverse biased. 
Step 2) The circuit on the right side of Figure 6.4 shows the reverse bias model. Notice the + side of the Multimeter that is measuring $V_{D 2}$ is on the same node as the anode was connected. To solve for VD2 there are two possible methods.

- Find the node voltage on the + and - sides of the open circuit and subtract them. $V_{D 2+}=0$ (grounded) and $V_{D 2-}=12-I \cdot R=12-0 \cdot R=12 \mathrm{~V}$ (current $=0$ A due to open circuit). $V_{D 2}=V_{D 2+}-V_{D 2-}=\underline{-12 ~ V}$

- Do a KVL around the loop including VD2 $\rightarrow-12 \mathrm{~V}+\mathrm{I} \cdot \mathrm{R}-\mathrm{V}_{\mathrm{D} 2}=0 \rightarrow \mathrm{V}_{\mathrm{D} 2}=\underline{-12 \mathrm{~V}}$

- $\mathrm{V}_{\mathrm{D} 2}=-12 \mathrm{~V}$ is verified in Multisim in Figure 6.5.

Step 3) $V_{D 2}<0.7$ so the reverse bias condition is verified ( $0.7 \mathrm{~V}$ is used here because it is a silicon diode) Step 4) The reverse bias condition assumed in Step 1) is verified in Step 3) so the problem is done.

Example 6.3) Follow the DC Diode Analysis process to solve the following circuit if D1 is a silicone diode.
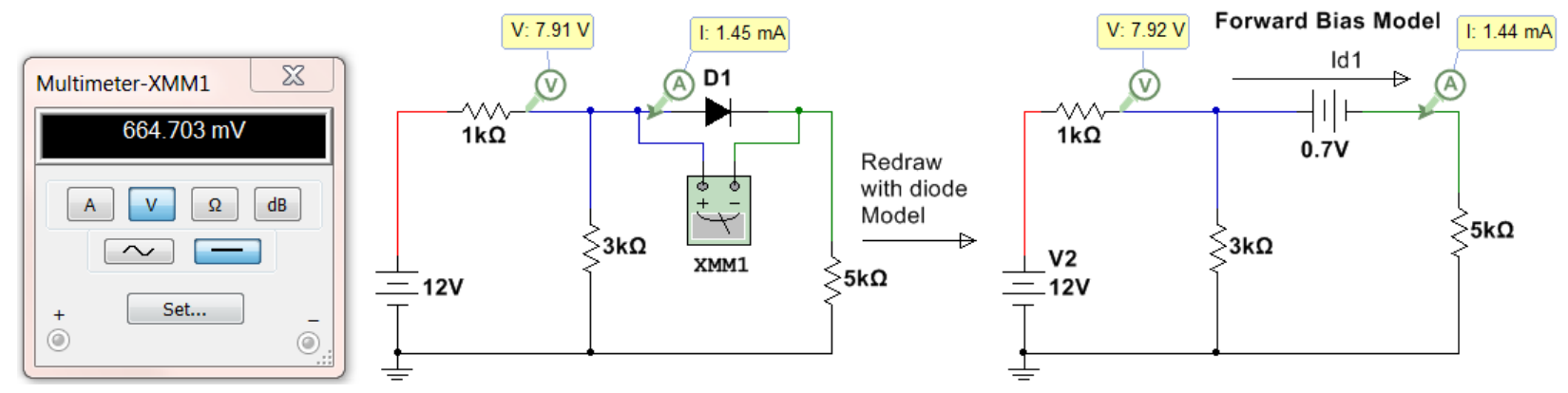

Figure 6.6: Circuit for Example 6.3

Step 1) D1 will be assumed to be forward biased because the $12 \mathrm{~V}$ source will force current clockwise in this circuit, which is the direction from the anode to cathode of the diode.

Step 2) The circuit on the right side of Figure 6.6 shows the forward bias model replacing the diode. The node where the anode was connected is connected to the + side of the battery. $0.7 \mathrm{~V}$ is used for the battery voltage because the problem stated it is a silicone diode. Next, calculate the current, $I_{D 1}$. The mesh matrix method used in the DC Circuits eBook will be used.

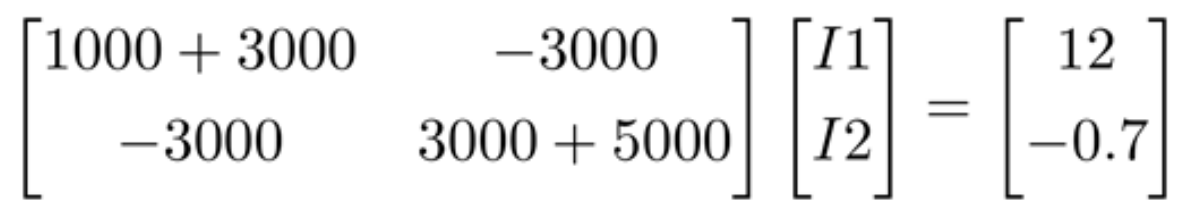

- Solving this matrix yields $12=\mathrm{I}_{\mathrm{D} 1}=\underline{\mathbf{1 . 4 4} \mathrm{mA}}$ (This is verified in Multisim in the right circuit of Figure 6.6)

- Note: The actual diode voltage is shown in Multimeter XMM1 to be $664.703 \mathrm{mV}$. The $0.7 \mathrm{~V}$ diode drop assumption yielded a slightly different result than the actual values (shown in the left circuit in Figure 6.6).

Step 3) Verify the diode is forward biased by making sure that $I_{D 1}>0$ and flowing from anode to cathode. The current is $1.44 \mathrm{~mA}$ and the direction is clockwise in the $2^{\text {nd }}$ loop (which is from the anode to cathode).

Step 4) The forward bias condition assumed in Step 1) is verified in Step 3) so the problem is done. 
Example 6.4) Follow the DC Diode Analysis process to solve the following circuit with a green LED.

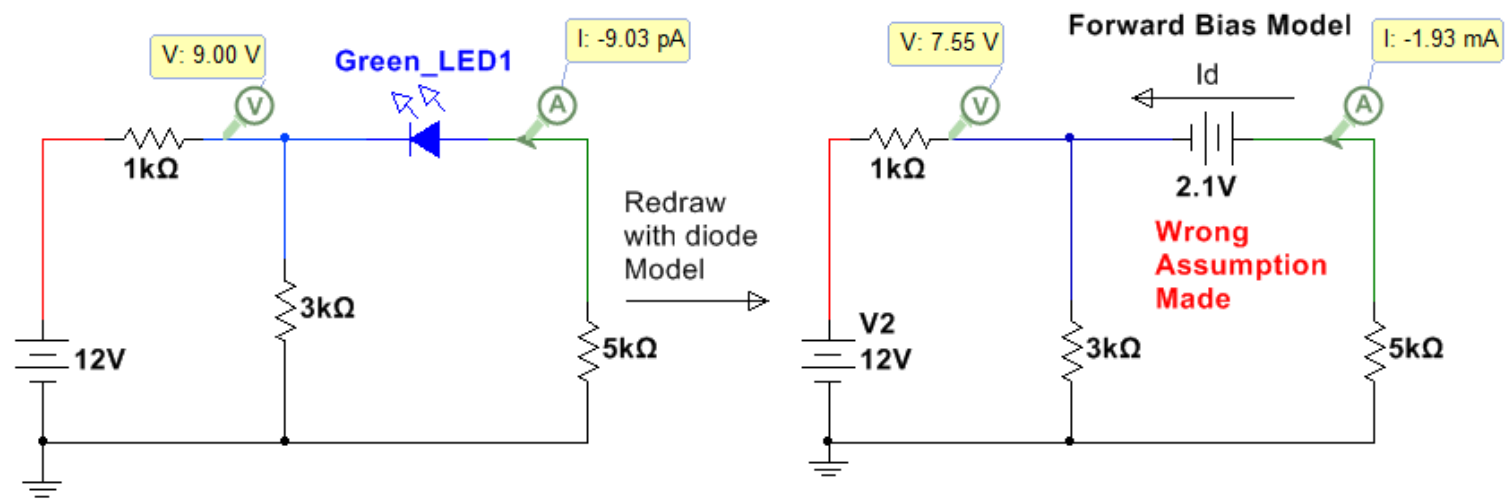

Figure 6.7: Circuit for Example 6.4 with the wrong assumption made.

Step 1) The LED will be assumed to be forward biased. It is fairly obvious that this is the wrong assumption, but it will be done anyway to show how to restart the problem in step 4 if the assumption is wrong.

Step 2) Solving for $I_{D}$ (which is the counterclockwise current in the $2^{\text {nd }}$ loop)

$$
\left[\begin{array}{cc}
1000+3000 & -3000 \\
-3000 & 3000+5000
\end{array}\right]\left[\begin{array}{c}
I 1 \\
I 2
\end{array}\right]=\left[\begin{array}{c}
12 \\
2.1
\end{array}\right]
$$

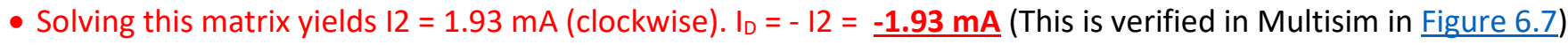

- Note: The probe values in the left circuit of Figure 6.7 with the green LED are way different because the wrong assumption was made.

Step 3) $I_{D}$ is flowing from cathode to anode so the forward biased assumption cannot be verified.

Step 4) The forward bias condition assumed in Step 1) is WRONG in Step 3) so start over at step 1.

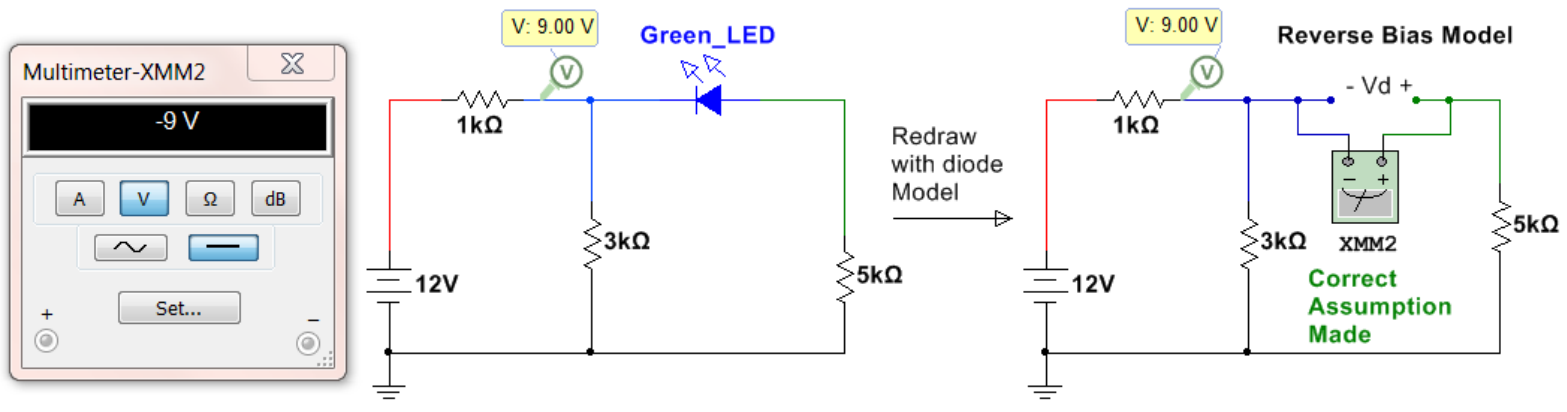

Figure 6.8: Circuit for Example 6.4 with the correct assumption made.

Redo Step 1) The LED will be assumed to be reverse biased. Since forward was wrong this must be correct.

Redo Step 2) Doing a KVL around the outer loop. $-12+11 \cdot 1 \mathrm{k} \Omega-\mathrm{V}_{D}+12 \cdot 5 \mathrm{k} \Omega=0$, All current flows through the first loop so I $1=12 /(4 \mathrm{k} \Omega)=3 \mathrm{~mA}$ and I2 $=0 \mathrm{~A} \rightarrow \mathrm{V}_{\mathrm{D}}=-12+3 \mathrm{~mA} \cdot 1 \mathrm{k} \Omega+0 \cdot 5 \mathrm{k} \Omega=\underline{-9} \mathrm{~V}$ (Verified in Multisim)

Redo Step 3) $V_{D}=-9 V<2.1$ V so reverse bias is verified.

Redo Step 4) The reverse bias condition assumed in Step 1) is verified in Step 3) so the problem is done. 
Example 6.5) Follow the DC Diode Analysis process to solve the following left circuit in Figure 6.9.

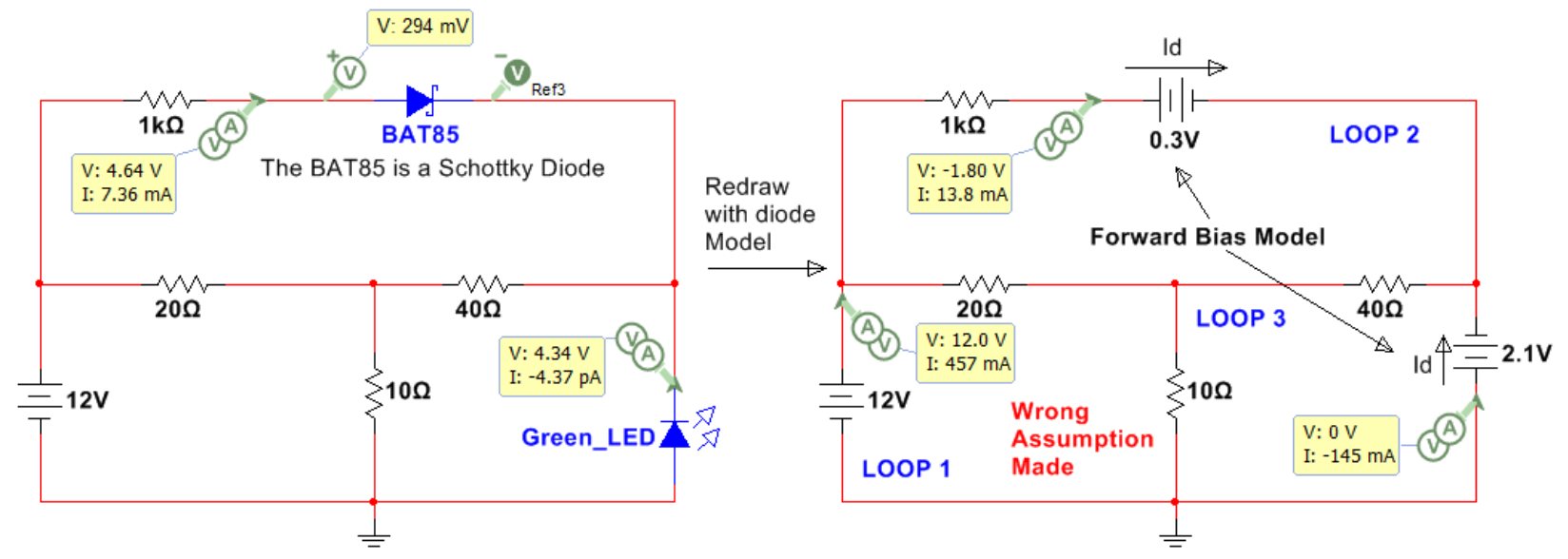

Figure 6.9: Circuit for Example 6.5 with the wrong assumptions made.

Step 1) Assume Both diodes are forward biased (1 of 4 possibilities). Model is shown in Figure 6.9 (Right).

Step 2) Solving for the loop currents. Assuming currents are clockwise $\rightarrow$ I_Bat85 = I2 and I_LED = -I3

\begin{tabular}{|c|c|c|c|c|c|c|}
\hline- & -20 & & {$[I 1]$} & & & Assuming currents are clockwise, solving this matrix yields \\
\hline-20 & $20+1000+40$ & -40 & 12 & $=$ & -0.3 & $\rightarrow I 1=457.4 \mathrm{~mA}, \mathrm{I} 2=13.8 \mathrm{~mA}, \mathrm{I} 3=144.5 \mathrm{~mA}$. (These values \\
\hline-10 & -40 & $10+40$ & {$[3]$} & & 2.1 & 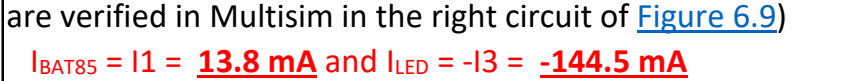 \\
\hline
\end{tabular}

Step 3) $I_{\text {BAT85 }}$ is flowing from anode to cathode so the forward biased assumption is verified, but the ILED is flowing from the cathode to anode so forward bias cannot be verified.

Step 4) If either diode is assumed wrong, one of the other 4 possibilities must be tried. There is a good chance the BAT85 is forward biased, but you can't be sure until you verify both are correct. Go back to step 1.

Redo Step 1) The LED will be assumed to be reverse biased and the BAT85 assumed to be forward biased.

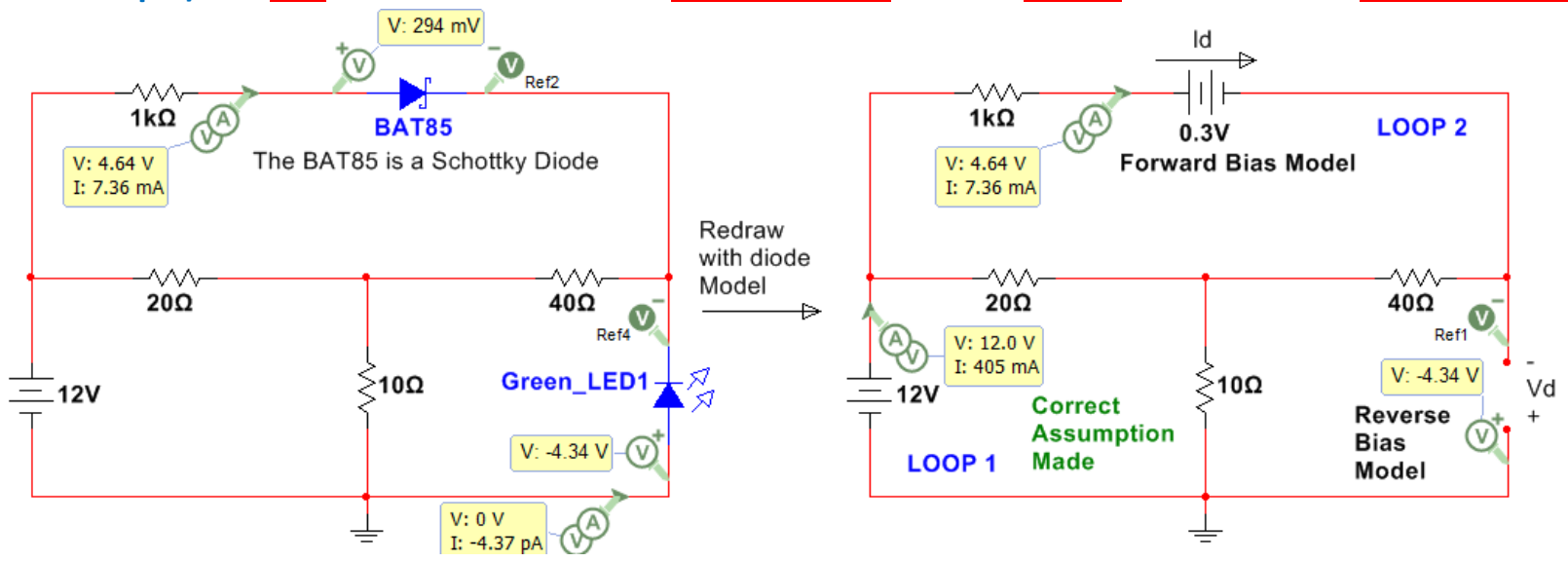

Figure 6.10: Circuit for Example 6.5 with the correct assumption made.

Redo Step 2) Loop 3 has an open circuit so this becomes a 2-loop circuit.

$\left[\begin{array}{cc}20+10 & -20 \\ -20 & 20+1000+40\end{array}\right]\left[\begin{array}{c}I 1 \\ I 2\end{array}\right]=\left[\begin{array}{c}12 \\ -0.3\end{array}\right] \quad \begin{aligned} & \text { Solving yields } \rightarrow I 1=404.9 \mathrm{~mA}, 12=I_{\text {BAT85 }}=\underline{7.36 \mathrm{~mA}} \\ & \mathrm{KVL} \text { around outer loop } \rightarrow-12+12 \cdot 1 \mathrm{k}+0.3-\mathrm{V}_{\mathrm{D}}=0 \\ & \mathrm{~V}_{\mathrm{D}}=-12+7.36 \mathrm{~mA} \cdot 1 \mathrm{k}+0.3=\underline{-4.34 \mathrm{~V}} \text { (verified in Multisim) }\end{aligned}$

Redo Step 3) $V_{D}=-4.34 \mathrm{~V}<2.1 \mathrm{~V}$ (reverse verified), $\mathrm{I}_{\mathrm{BAT}} \mathrm{T5}=\underline{7.36 \mathrm{~mA}}$ from anode to cathode (forward verified)

Redo Step 4) The assumptions in redo Step 1) is verified in Step 3) so the problem is done. 


\section{Section 6.2 - Diode Logic Circuits}

There are many applications where the basic diode calculations shown in Section 6.1 can be used to give circuitry more control. One way is by using diodes to create logic gates. The circuit in Figure 6.11 shows diodes acting as an OR gate. To make this section easier to understand Multisim virtual parts are used that show actual images of the part. When either of the two switches is flipped to the ON position (up) then the LED will turn on. When the switch is in the down position there is an open circuit connected to the diode so a pull down resistor is used to make sure the voltage at the diode is OV when the switch is in the OFF position and $5 \mathrm{~V}$ when the switch is in the ON position. The left circuit has both switches OFF and the right circuit has Switch 2 ON. Switch 1 could have been turned on instead of Switch 2 (or both could be turned on) and the same output would occur.
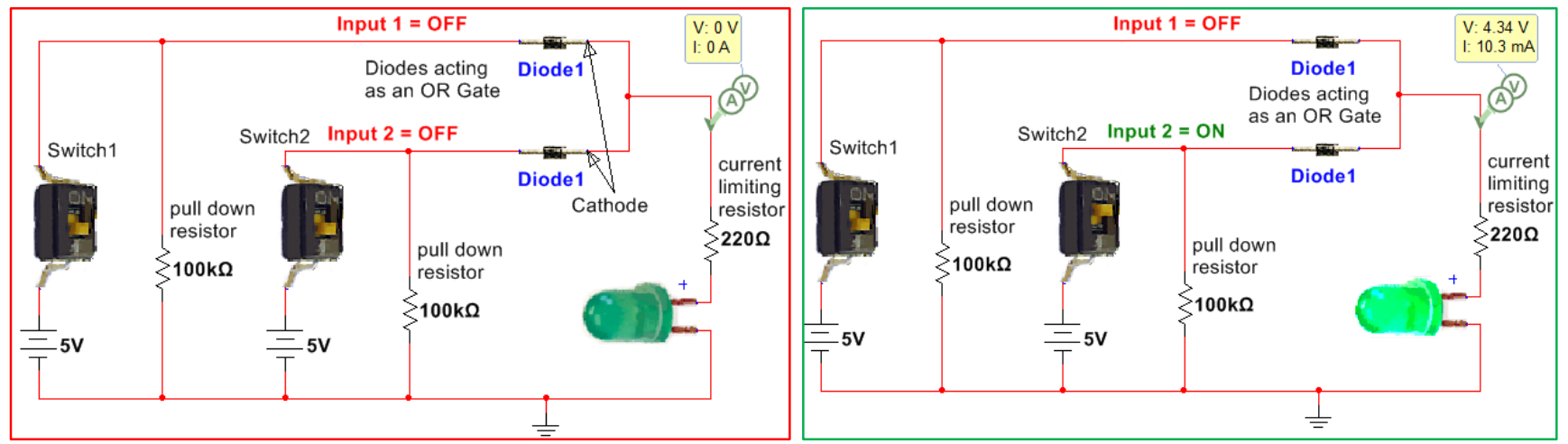

Figure 6.11: Diodes acting as an OR GATE.

The circuit in Figure 6.12 shows diodes acting as an AND gate. The output voltage (Vout) will only go to $5 \mathrm{~V}$ when both switches are flipped to the ON position (up) as shown in Figure 6.12. When either switch is turned off the voltage will drop across the resistor that sets the voltage levels (200 $\mathrm{k} \Omega$ in Figure 6.12). The higher this resistor value is the lower the current will be and the lower the voltage will be when both switches aren't pressed. In this case with a $200 \mathrm{k} \Omega$ resistor used when either switch is ON Vout drops below $2 \mathrm{~V}$ which is the minimum voltage required by the gate of the MOSFET to turn it on and cause current to flow from the $5 \mathrm{~V}$ rail through the drain.

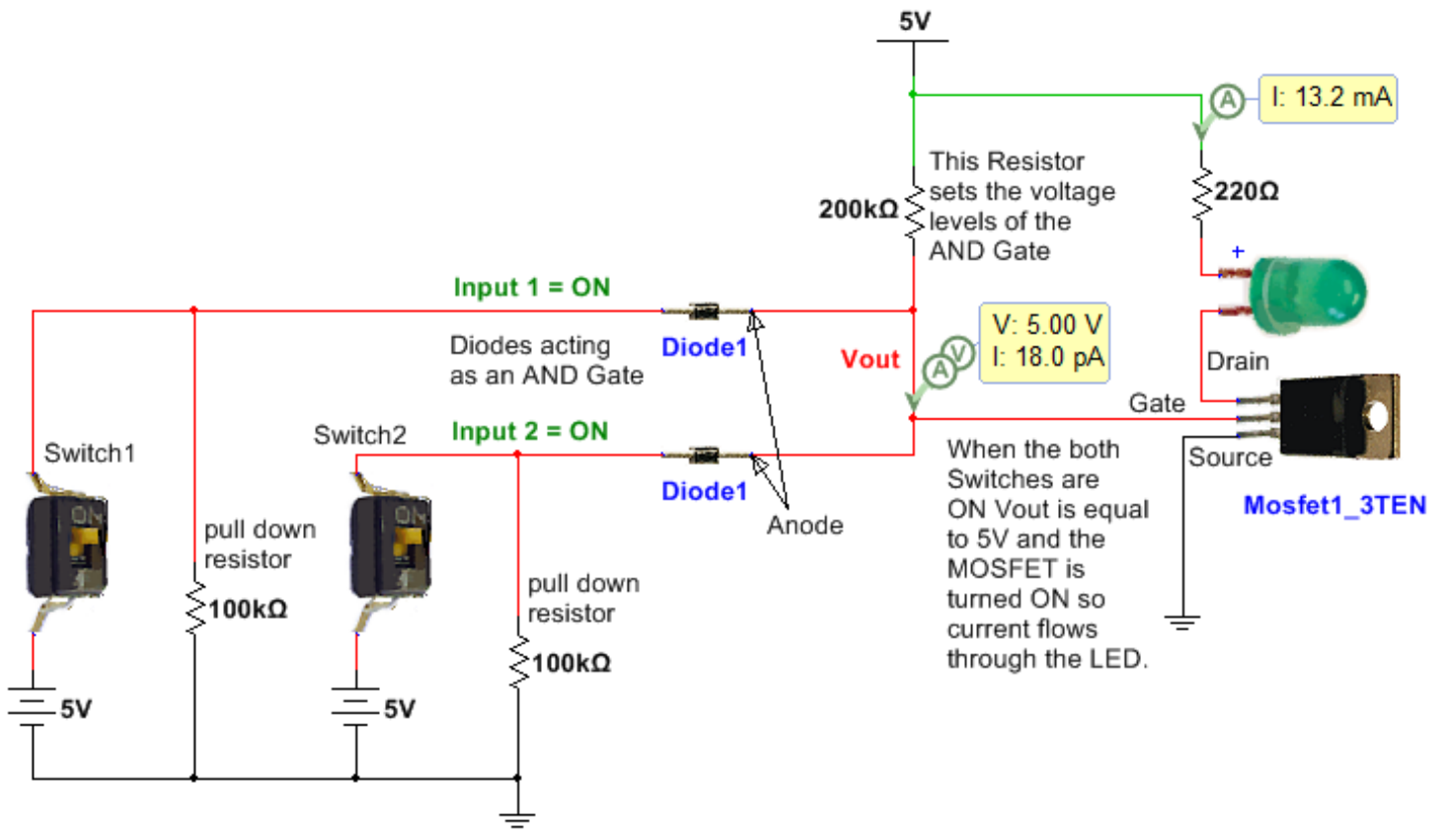

Figure 6.12: Diodes acting as an AND GATE. 


\section{Section 6.3 - Protection Diodes}

One of the most important uses of diodes is to protect circuitry from voltage spikes that occur when inductive elements such as motors start or stop. "Protection diodes" that serve this function are referred to by many different names. Some of these names are: flyback, flywheel, freewheeling, snubber, and catch diodes. The magnitude of the voltage across an inductor is $V_{L}=L \frac{d i}{d t}$ so when the inductive load is turned on or off the current changes from 0 to non-zero producing a large slope and in return a large voltage spike. Because MOSFET transistors (as shown in Figure 6.12) are activated with only voltage signals (Note: current is required to activate BJT transistors) they are especially susceptible to damage when turning on or off an inductive load. A diode can be positioned across the inductive load so that the voltage spike doesn't reach the circuitry as shown in Figure 6.13. The protection diode could also be placed across the MOSFET instead of the motor to provide protection for the MOSFET. For this situation, the anode of the diode would connect to the source and the cathode would connect to the drain. The protection diode needs to be a fast switching style diode like a 1N914.

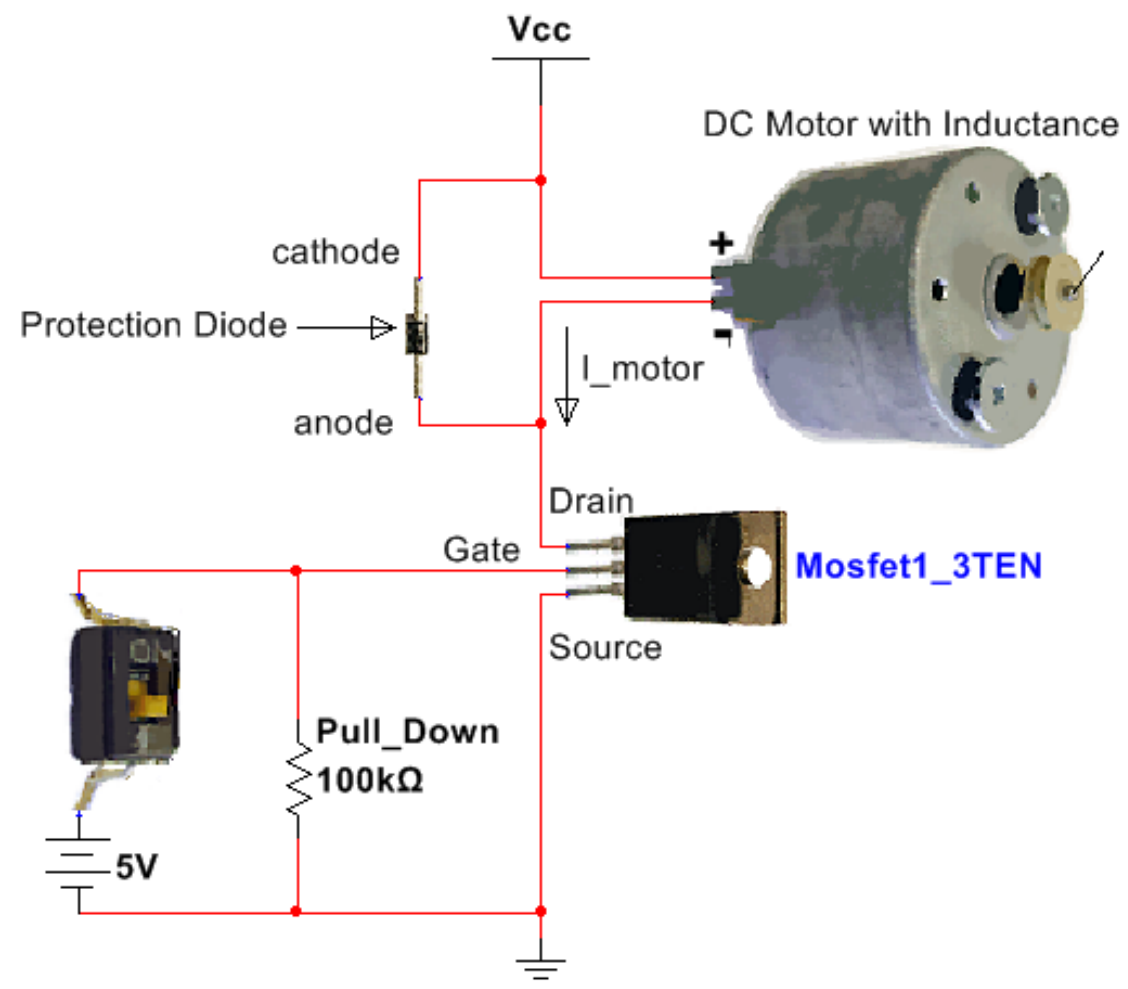

Figure 6.13: Using a protection diode to prevent damaged circuitry due to $V_{L}=L \frac{d i}{d t}$

Protection from $V_{L}=L \frac{d i}{d t}$ is such a problem for MOSFETs that some have internal protection as shown in the following figure taken from the BS170 datasheet. Even if a diode has internal protection it is usually not enough to keep the MOSFET from be damaged so adding an external protection diode as shown in Figure 6.13 is recommended.

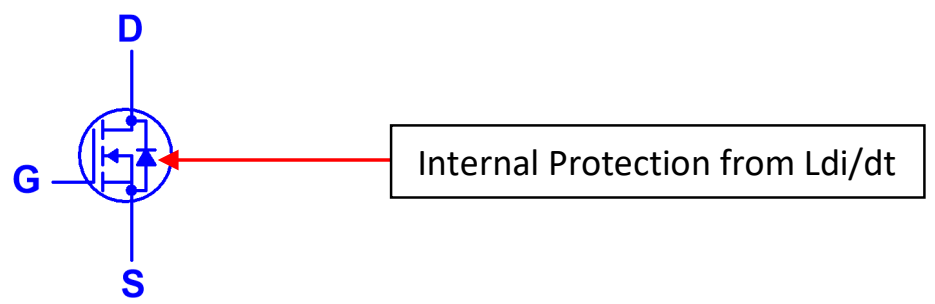

Figure 6.14: Internal protection in a BS170 MOSFET. 


\section{Section 6.4 - Rectifiers}

One of the most important uses of diodes is to rectify an AC signal so it can be converted to a DC signal. When the diode is connected in series between an AC signal and a resistor only one side of the AC waveform can pass through. If the diode is oriented as shown in Figure 6.15 only the positive cycles are passed through, but if the diode is flipped only the negative cycles pass through. The circuit in Figure 6.15 shows a half-wave rectifier circuit. It is called half-wave because only half of the cycles pass through and the other half are rejected (or zeroed out). For this circuit the input voltage is $2 \mathrm{Vrms}$ or $2.8 \mathrm{Vpk}$. The peak input voltage to rectifier circuit is typically called Vs because it is usually connected to the secondary of a transformer. The peak voltage across the resistor is called Vo,pk and is one diode drop less than Vs for a half-wave rectifier with only one diode. The reason the "with only one diode" disclaimer was used in the previous sentence is due to the fact that sometimes multiple diodes will be included in series to get Vo,pk to the desired level. In the circuit in Figure 6.15 Vs is measured by Multisim as 2.807 and Vo,pk was measured as $2.234 \mathrm{~V}$ which makes the diode drop around $0.6 \mathrm{~V}$ instead of the $0.7 \mathrm{~V}$ that we will assume in all rectifier calculations that use silicone rectifier diodes. Keep this in mind as one of the sources of error when Multisim doesn't match up as closely to rectifier hand calculations as desired. If the peak output voltage (Vo,pk) needed to be lower than $2.234 \mathrm{~V}$ then another diode could be placed in series with the $1 \mathrm{~N} 4007 \mathrm{G}$ diode to reduce it by another 0.6 or 0.7 volts.
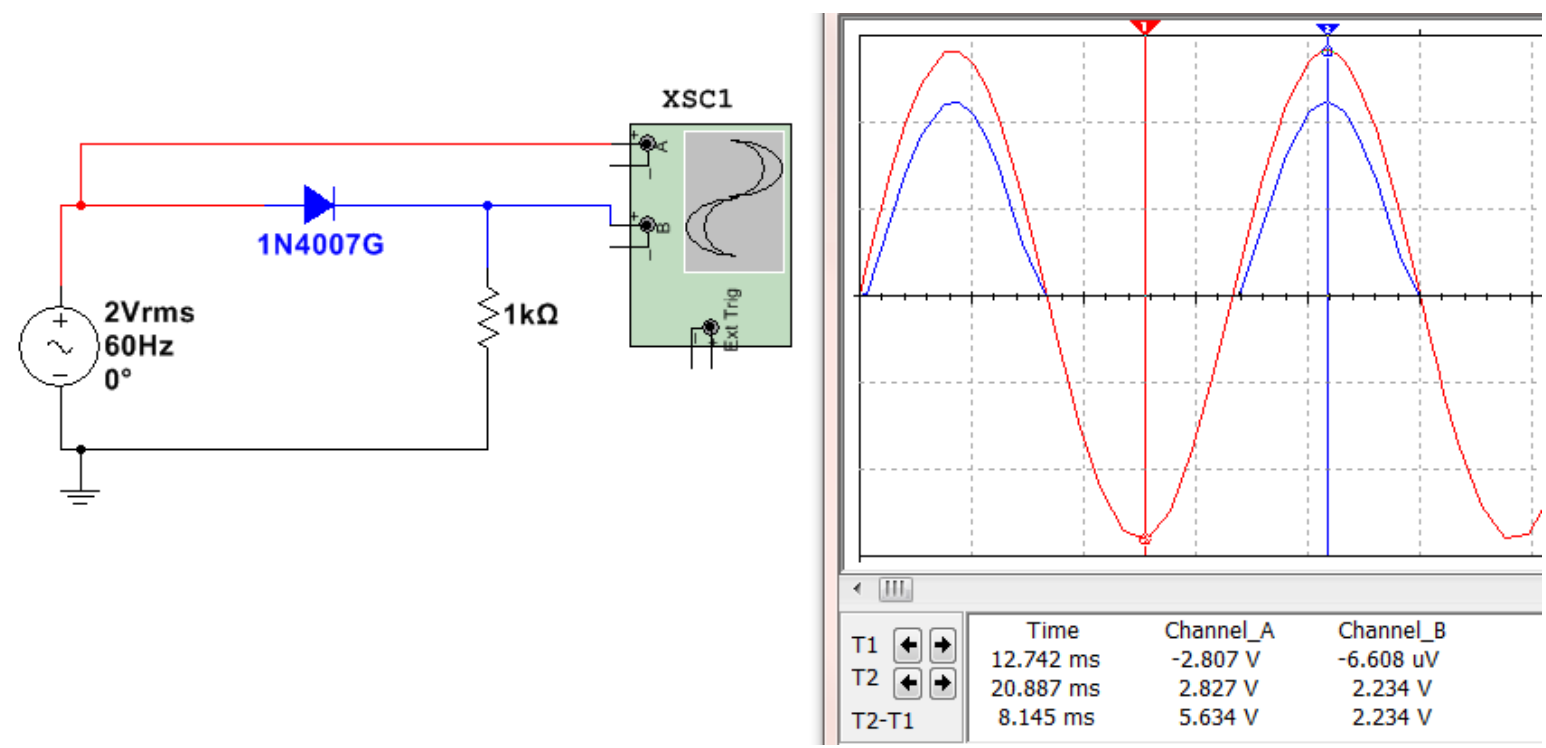

Figure 6.15: Half-Wave Rectifier with one of the 1 N4000 series silicone rectifier diodes.

Rectification is the first step in converting an AC signal to a DC signal. The next step would be to add a capacitor in parallel with the load resistor where Vo,pk is measured so that the voltage will not be allowed to drop very fast due to the time constant of the capacitor. Once the "smoothing capacitor" is added the rectifier is called a "Peak Rectifier". An example of a half-wave peak rectifier is shown in Figure 6.16. The calculations and Multisim measurements of a half-wave peak rectifier are shown below (See equation summary in Table 6.1).

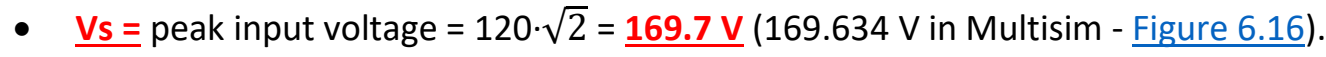

- $\underline{\text { Vo,pk }}=$ peak output voltage $=\mathrm{Vs}-0.7 \mathrm{~V}=\underline{169 \mathrm{~V}}(169.026 \mathrm{~V}$ in Multisim $-\underline{\text { Figure 6.16 }})$.

- $\underline{\mathrm{Vr}}=$ ripple voltage $(\mathrm{Vo}, \mathrm{pk}-$ minimum $\mathrm{Vout})=\frac{V_{O, p k}}{f R C}=\frac{169}{60 \cdot 50 \mathrm{k} \cdot 1 \mu}=\underline{56.3 \mathrm{~V}}(\sim 41.3 \mathrm{~V}$ in Figure 6.16).

- $\quad \underline{\mathrm{Vr}}=$ percent ripple $=$ the ripple voltage divided by $\mathrm{Vo}, \mathrm{pk}=(56.3 / 169)=33.3 \%$

- $\underline{\mathrm{V}}_{\mathrm{O}, \mathrm{DC}}=$ the approximate $\mathrm{DC}$ voltage (centerline of the ripple). In Figure $6.16 \mathrm{~V}_{\mathrm{O}, \mathrm{DC}}$ is equal $169-0.5 \cdot \mathrm{Vr}=$ $169-0.5 \cdot 41.3=\underline{\mathbf{1 4 8 . 4 \mathrm { V }}}$. The DC voltage probe in Figure 6.16 measures the DC voltage as $149 \mathrm{~V}$. 

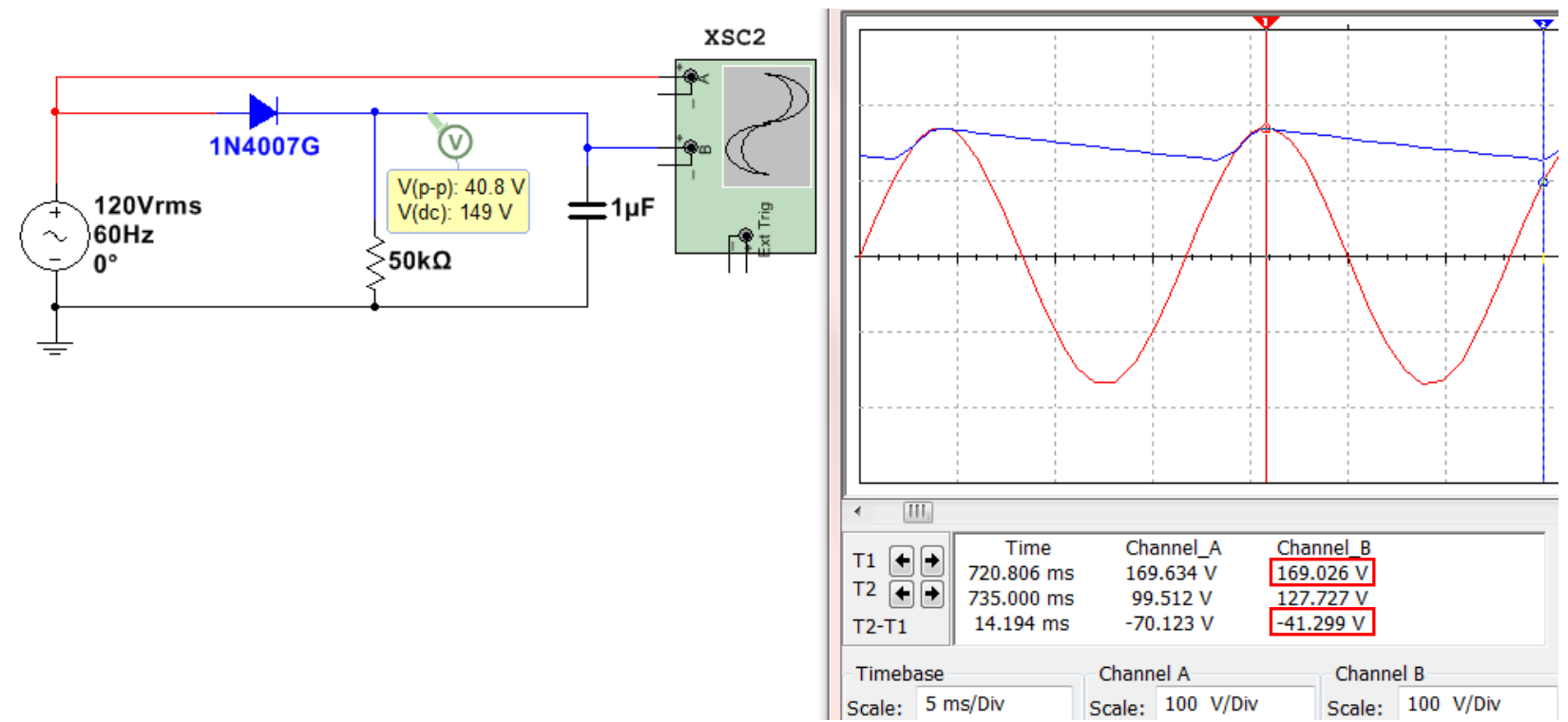

Figure 6.16: Half-Wave Peak Rectifier.

The other type of rectifier is called a full-wave rectifier. It allows the negative peaks to be flipped to positive instead of zeroing them out. There are two common types of full-wave peak rectifiers:

- Full-Wave Peak Bridge Rectifier (See Figure 6.17)

- Full-Wave Peak Parallel Rectifier or Full-Wave Peak Center Tapped Rectifier (This type will not be discussed in this eBook, but the equations are in Table 6.1 and the circuit can be seen in the third figure from the top in this link).
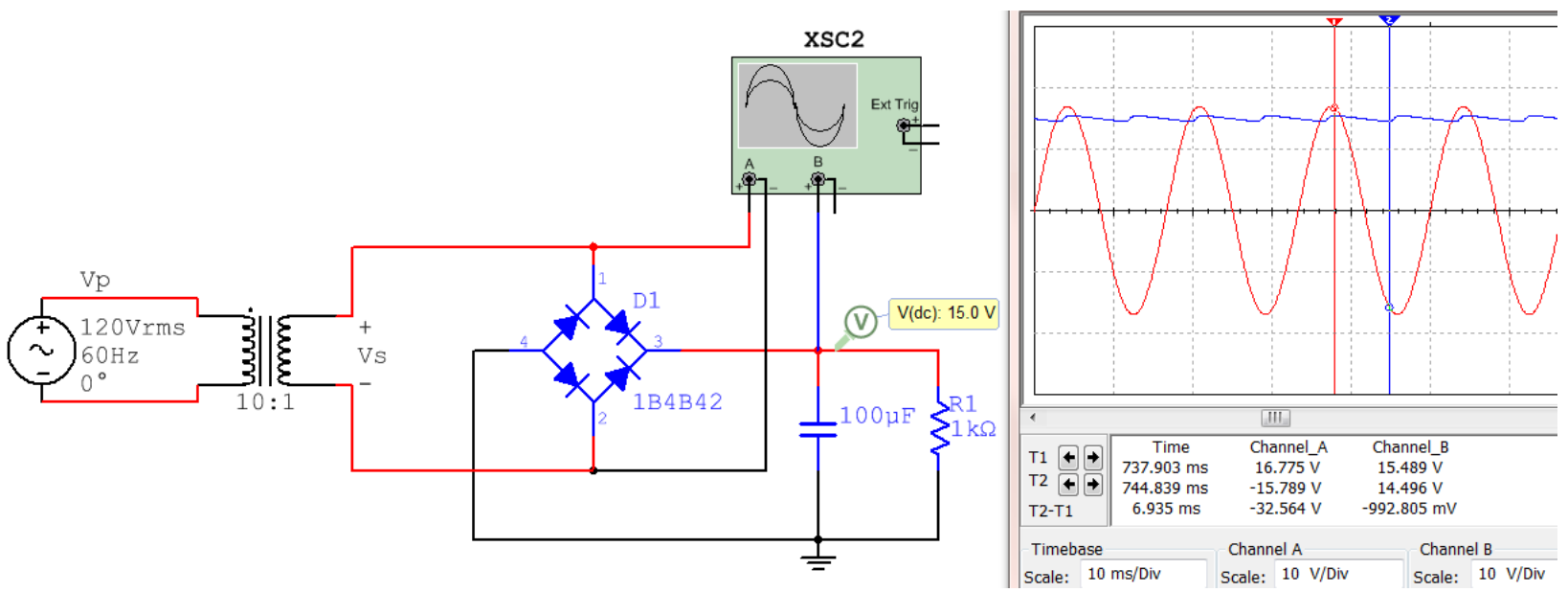

Figure 6.17: Full-Wave Peak Bridge Rectifier.

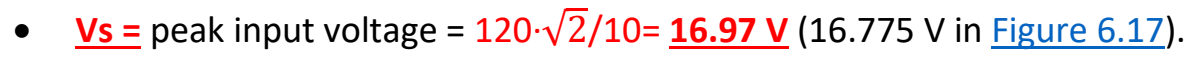

- $\underline{\text { Vo,pk }}=$ peak output voltage $=\mathrm{Vs}-2 \cdot 0.7 \mathrm{~V}=\underline{15.57 \mathrm{~V}}(15.49 \mathrm{~V}$ in Multisim $-\underline{\text { Figure 6.17) }}$.

- $\underline{\mathrm{Vr}}=$ ripple voltage $(\mathrm{Vo}, \mathrm{pk}-$ minimum Vout $)=\frac{V_{O, p k}}{2 f R C}=\frac{15.57}{2 \cdot 60 \cdot 1 \mathrm{k} \cdot 100 \mu}=\underline{1.3 \mathrm{~V}}(\sim 1 \mathrm{~V}$ in Figure 6.17)

- $\quad \underline{\mathrm{V} r}=$ percent ripple $=$ the ripple voltage divided by $\mathrm{Vo}, \mathrm{pk}=(1.3 / 15.57)=8.3 \%$

- $\underline{\mathrm{V}}_{\mathrm{O}, \mathrm{DC}} \equiv$ the approximate $\mathrm{DC}$ voltage of the output (centerline of the ripple). In Figure $6.17 \mathrm{vo,DC}$ is equal to $15.57-0.5 \cdot \mathrm{Vr}=\underline{14.92 \mathrm{~V}}$. The DC voltage probe in Figure 6.17 measures the DC voltage as $15 \mathrm{~V}$. 
The equations for half-wave and full-wave peak rectifiers that were shown in Figures 6.16 and 6.17 are summarized in Table 6.1. Note: The Full Wave Center Tapped equations are shown, but this type of rectifier circuit is not covered in detail in this eBook.

Table 6.1: Peak Rectifier Summary, Note: $\mathrm{V} \boldsymbol{\mu}=\mathrm{Vo}$, pk

\begin{tabular}{|c|c|c|c|c|c|c|c|}
\hline Type & $\#$ & PRV & $V_{o, p k}$ & $\mathrm{~V}_{\mathrm{r}}$ & $V_{0, D C}$ & $i_{D, a v g}$ & $i_{D, \max }$ \\
\hline $1 / 2$ Wave & 1 & $\mathrm{~V}_{\mathrm{S}}$ & $V_{S}-V_{D}$ & $\mathrm{~V}_{\mu} /\left(\mathrm{f}_{\mathrm{i}} \mathrm{RC}\right)$ & $V_{\mu}-1 / 2 V_{r}$ & $\mathrm{I}_{L}\left[1+\pi\left(2 \mathrm{~V}_{\mu} / \mathrm{V}_{\mathrm{r}}\right)^{1 / 2}\right]$ & $\mathrm{I}_{L}\left[1+2 \pi\left(2 \mathrm{~V}_{\mu} / \mathrm{V}_{\mathrm{r}}\right)^{1 / 2}\right]$ \\
\hline Full-Wave, Bridg & 4 & VS - VD & VS - 2VD & $\mathrm{V} \mu /(\underline{\mathbf{2}} \mathrm{fiRC})$ & $V \mu-1 / 2 V r$ & $\mathrm{IL}[1+\pi(\mathrm{V} \mu / 2 \mathrm{Vr}) \mathrm{1} / 2]$ & $\mathrm{IL}\left[1+2 \pi(\mathrm{V} \mu / 2 \mathrm{Vr})^{1 / 2}\right]$ \\
\hline Full Wave, C.T. & 2 & $2 V_{S}-V_{D}$ & $V_{S}-V_{D}$ & $V_{\mu} /\left(\underline{\underline{2}} f_{i} R C\right)$ & $V_{\mu}-1 / 2 V_{r}$ & $\mathrm{I}_{L}\left[1+\pi\left(\mathrm{V}_{\mu} / 2 \mathrm{~V}_{\mathrm{r}}\right)^{1 / 2}\right]$ & $\mathrm{I}_{L}\left[1+2 \pi\left(\mathrm{V}_{\mu} / 2 \mathrm{~V}_{\mathrm{r}}\right)^{1 / 2}\right]$ \\
\hline
\end{tabular}

Nomenclature / Notes for Table 6.1

- $1 / 2$ Wave $=1 / 2$ Wave Peak Rectifier ( 1 diode, 1 resistor, 1 capacitor)

- Full Wave, C.T. = Full Wave Center Tapped Peak Rectifier (2 diodes, 1 resistor, 1 capacitor)

- Full Wave, Bridge = Full Wave Bridge Peak Rectifier (4 diodes, 1 resistor, 1 capacitor)

- $\quad \#=$ Number of Diodes in the circuit.

- $V_{s}=$ Peak input voltage (if there is a transformer, this is the secondary voltage) NEVER use Vrms for Vs.

- $\mathrm{V}_{\mu}=\mathrm{V}_{\mathrm{o}, \mathrm{pk}}=$ Peak output voltage across the load resistor

- $V_{D}=$ Diode drop (Depends on the current, but we will assume $0.7 \mathrm{~V}$ for calculations (See Figure 6.2)

- $V_{r}=$ Ripple Voltage. The difference between $V_{0}, p k$ and the minimum of the rectified/filtered Vout signal.

$>$ Note: $V_{r}=V_{\mu}$ if it is NOT a peak rectifier (i.e. no capacitor).

- $f_{i}=$ The frequency of the input signal (usually $60 \mathrm{~Hz}$ in U.S.)

- $\mathrm{R}=$ Load Resistor

- $\mathrm{C}=$ Capacitor that is in parallel with the load resistor

- $\mathrm{V}_{\mathrm{O}, \mathrm{DC}}=$ The $\mathrm{DC}$ (or average) voltage across the load resistor. (This is typically what you design for). The approximate equation for $V_{0, D C}$ in Table 6.1 is more accurate the smaller the value of $\mathrm{Vr}$.

- $\quad P R V=$ Peak Reverse Voltage for each of the diodes (also called PIV, Peak Inverse Voltage).

$>$ For a sinusoid input this calculated value must be $<$ the peak repetitive reverse voltage limit found on the spec. sheet. For the 4000 series rectifier diodes the PRV limit increases from 1N4001 to 4007.

$>$ The continuous reverse voltage limit on the spec. sheet is referring to a situation where you have a large DC value being blocked by the diode.

- $\quad \mathrm{i}_{\mathrm{D}, \mathrm{avg}}=$ Average current of the diode. This value should be less than the average forward rectified current limit on the spec. sheet.

- $\quad \mathrm{i}_{\mathrm{D}, \max }=$ Peak current of the diode. This value should be less than the maximum forward rectified current limit on the spec. sheet.

- $\mathrm{I}_{\mathrm{L}}=\mathrm{V}_{\mathrm{O}, \mathrm{DC}} / \mathrm{R}=$ This is approximately the average current through the load resistor.

Example 6.6) Calculate the ripple voltage, \%Vr, and approximate DC voltage for the Full Wave bridge peak rectifier in Figure 6.18. Assume all diodes have voltage drops of 0.7 Volts.

The circuit in Figure 6.18 shows the Full Wave bridge peak rectifier drawn in two different ways. The left circuit requires a diode bridge to be used (4 diodes in one package configured as a bridge). The right circuit is much more convenient and useful because it allows for specific individual diodes (such as the 1N4007) to be used. 


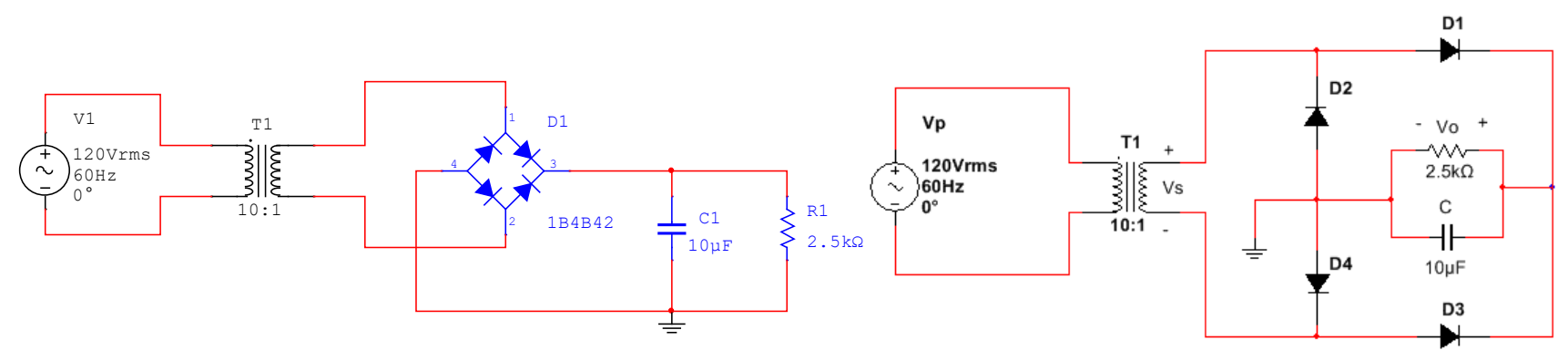

Figure 6.18: Full-Wave Peak Bridge Rectifier for Example 6.6.

- $\quad \mathrm{Vin}, \mathrm{rms}=\mathrm{Vp}, \mathrm{rms}=120 \mathrm{Vrms}$

- $\quad \mathrm{Vin}, \mathrm{pk}=\mathrm{Vp}, \mathrm{pk}=120 *$ sqrt $(2)=169.7 \mathrm{~V}$

- $V s, p k=169.7 /($ Turns ratio $)=169.7 /(\mathrm{Np} / \mathrm{Ns})=169.7 / 10=16.97 \mathrm{~V}$

- $\quad \mathrm{Vo}, \mathrm{pk}=\mathrm{Vs}, \mathrm{pk}-2 * 0.7 \mathrm{~V}=15.6 \mathrm{~V}$

- $\mathrm{Vr}=\mathrm{Vo}, \mathrm{pk} /\left(\underline{\mathbf{2}} * \mathrm{f}_{\mathrm{i}}^{*} \mathrm{R} * \mathrm{C}\right)=15.6 /(\underline{\mathbf{2}} * 60 * 2500 * 10 \mathrm{e}-6)=5.2 \mathrm{~V}$ ( $\underline{\text { Note: }}$ The $\underline{\mathbf{2}}$ is used for a full wave rectifier)

- $\quad \% \mathrm{Vr}=\mathrm{Vr} / \mathrm{Vo}, \mathrm{pk}=5.2 / 15.6=\underline{\mathbf{3 3} .3 \%}$

- $\mathrm{V}_{\mathrm{O}, \mathrm{DC}}=\mathrm{Vo}, \mathrm{pk}-1 / 2(\mathrm{Vr})=15.6-1 / 2(5.2)=\underline{13 \mathrm{~V}}$

Example 6.7) Determine the turns ratio of the transformer and the capacitor value needed if you wanted to use a half-wave peak rectifier with a $1 \mathrm{~N} 4007$ diode to produce a DC voltage of $24 \mathrm{~V}$ with $10 \%$ ripple from a standard $120 \mathrm{Vrms} 60 \mathrm{~Hz}$ AC power outlet. You are required to use a load resistor of $100 \Omega$.

- $\quad$ Given $\rightarrow \% \mathrm{Vr}=10 \% \quad$ Equation 1) $\% \mathrm{Vr}=0.1=\mathrm{Vr} / \mathrm{Vo}, \mathrm{pk}$

- $\quad$ Given $\rightarrow V_{O, D C}=24 \quad$ Equation 2) $V_{O, D C}=24=V_{0}, p k-1 / 2(V r)$

- Solve equations 1 and 2 for $V o, p k$ and $V r \rightarrow 24=V_{0}, p k-1 / 2(0.1 \cdot V o, p k)=0.95 \mathrm{Vo}, p k \rightarrow \underline{V o, p k}=25.3 \mathrm{~V}$

- $\mathrm{Vr}=0.1 \cdot \mathrm{Vo}, \mathrm{pk} \rightarrow \underline{\mathrm{Vr}=2.53 \mathrm{~V}}$

- We need to select a transformer turns ratio that puts $V_{0}, p k=25.3$.

- $\mathrm{Vs}=\mathrm{Vo}, \mathrm{pk}+0.7 \rightarrow \mathrm{Vs}=26 \mathrm{~V}$

- Turns Ratio $=169.7 / 26 \rightarrow \underline{\mathrm{Np} / \mathrm{Ns}=\mathbf{6 . 5 2 7}}$ (At this point it would be best to select a common transformer you can purchase like a 6:1 and recalculate the $\mathrm{Vr}$ needed, but we will leave it as 6.527 in this example.

- $\mathrm{Vr}=2.53 \mathrm{~V}=\mathrm{Vo}, \mathrm{pk} /\left(\mathrm{f}_{\mathrm{i}}^{*} \mathrm{R}^{*} \mathrm{C}\right) \rightarrow 2.53 \mathrm{~V}=25.3 \mathrm{~V} /(60 * 100 * \mathrm{C}) \rightarrow \underline{\mathrm{C}=1.667 \mathrm{mF}}$

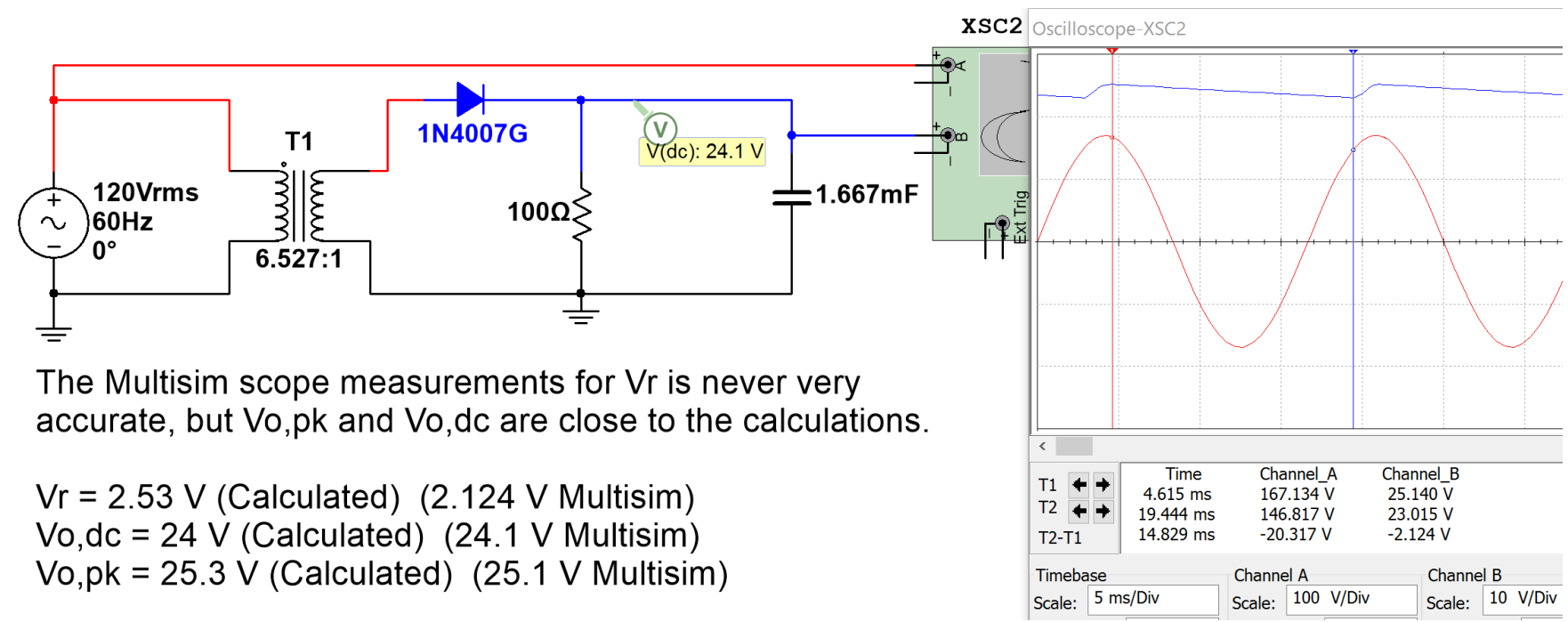

Figure 6.19: Full-Wave Peak Rectifier design for Example 6.7. 


\section{Module 7 -Operational Amplifiers (OpAmps)}

Operational Amplifiers (OpAmps) are integrated circuits (ICs) with a minimum of 5 pins. Sometimes multiple OpAmps are packaged together in a single IC. One example of this style of packaging is the LM324 Quad OpAmp. The two types of LM324 IC packages available are the 14 pin Dual Inline Package (DIP) and the 14 pin Small Outline Package (SOP). The pinout mapping and a photo of the DIP package are shown in Figure 7.1. The LM324 is an inexpensive general purpose (i.e. low power) OpAmp (see Figures 7.8 and 7.9 for more info). For details about this OpAmp read the datasheet at this link: https://www.fairchildsemi.com/datasheets/LM/LM324.pdf
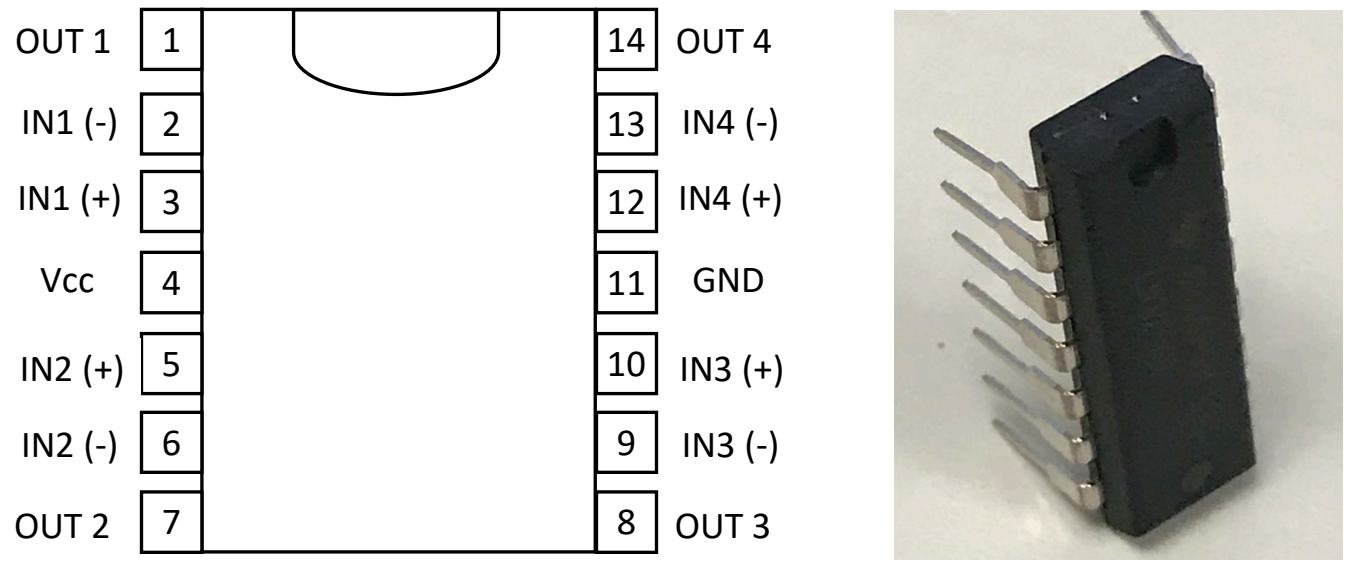

Figure 7.1: LM324 Quad OpAmp IC Pinout (Left), LM324 14-DIP package photo (Right)

The 14-DIP (Dual Inline Package) is called a "through-hole" part. Its pins can be plugged into a breadboard or into holes on a printed circuit board (PCB) or soldering proto board. The 14-SOP (Small Outline Package) is a "surface mount" part. It is one of the most popular surface mount IC packages and its 14 pins can only be connected by soldering them to pads on a PCB. The names for each of the 14 pins are shown in Figure 7.1. To minimize the number of pins required all 4 of the OpAmps use the same power pins. Pin 4 (Vcc) is called the positive rail and pin 11 (GND) can either be grounded for circuits only needing a single supply (where the output can go from $\mathrm{OV}$ to $\mathrm{Vcc}$ ) or connected to a negative voltage for circuits requiring a dual supply (where the output can go from $-V c c$ to $+V c c)$. The LM324 datasheet states that the limits for supplies for this OpAmp are: $(\mathrm{min}=$ $\pm 1.5 \mathrm{~V}, \max = \pm 16 \mathrm{~V}$ for a Dual Supply) or ( $\min =3 \mathrm{~V}, \max =32 \mathrm{~V}$ for single supply). Figure 7.2 shows the standard method of connecting power to an OpAmp in both dual and single supply options (Note that the rails must be reversed for Multisim simulations). OpAmps have 2 inputs (inverting or - input) and (non-inverting or + input) and one output (Vout). Note: There some additonal OpAmp example problems at the end of the Appendix.

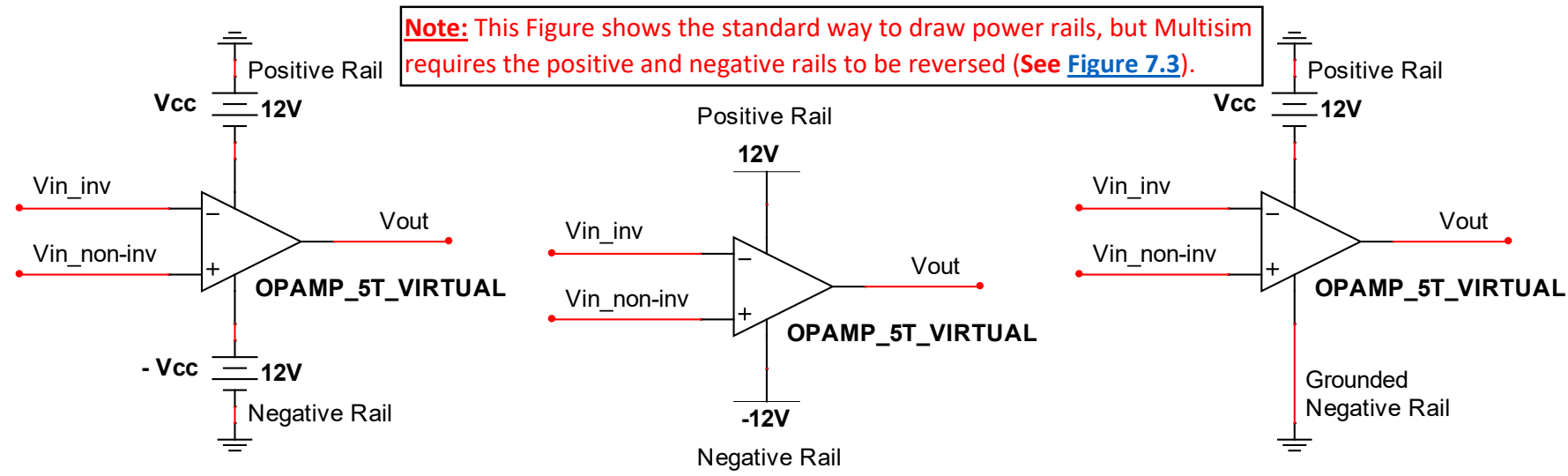

Figure 7.2: Dual Supply (Left), Dual Supply Alternate Form (Middle), Single Supply (Right). 
As noted in Figure 7.2 Multisim requires the positive and negative rails to be reversed from the standard way of drawing them. If you connect power rails as they are shown in Figure 7.2 Multisim gives the following error when running a simulation: "A simulation error has occurred. Would you like to run the Convergence Assistant to attempt to resolve this problem automatically?" If Yes is selected it takes a long time to try to fix the problem, but isn't able fix it. The way Multisim requires the power rails to be drawn is shown on the top right of Figure $\underline{7.3}$.

The OPAMP_5T_VIRTUAL in Multisim is a good part to use (and will be used for all circuits in this eBook) because the default internal parameters allow it to closely match hand calculations with the Ideal OpAmp Model (Explained in Section 7.2) and also allow easy adjustments of these parameters if needed. Double clicking on the OpAmp in Multisim brings up the interface in Figure 7.3 that can be used to set the OpAmp internal parameters. The 5 parameters that factor into whether or not an OpAmp can be assumed to be "ideal" are shown in the bottom right section of Figure 7.3. The parameters ( $\mathrm{A}, \mathrm{FU}, \mathrm{CMRR}, \mathrm{RI}$ ) that are labeled 1) through 4) in Figure 7.3 are assumed to be infinity for the Ideal Model and the values for those parameters in the OPAMP_5T_VIRTUAL are large enough to be approximated as infinity. The $5^{\text {th }}$ parameter is R0 which is $10 \Omega$ for the OPAMP_5T_VIRTUAL and assumed to be 0 for the Ideal Model. A $10 \Omega$ value is close enough to zero for the Ideal Model to give accurate results as long as the output of the OpAmp is not connected to a load resistance that is too small. If the load resistance is smaller than around $1 \mathrm{k} \Omega$ then it is best to set the RO value to a lower value (or even to 0 ) to closely match the Ideal Model calculations with Multisim. The "output short circuit current" parameter is the maximum allowable current that can be sourced or sinked by this OpAmp. In Figure $\underline{7.3}$ it is labeled with an * next to. Since it only has a $25 \mathrm{~mA}$ value it might need to be increased. If it is changed to a very large value (like $1000 \mathrm{Amps}$ ) then you won't have to worry about the current being limited internally by the OpAmp model in Multisim and the calculations will match the simulations more closely.

Learning about the details of the parameters in Figure 7.3 are important to gain a full understanding of OpAmps, but it will be left out in this eBook. A Texas Instrument (TI) white paper SLOA011 titled "Understanding Operational Amplifier Specifications" provides more information about the internal parameters of OpAmps. TI has an abundance of high quality technical literature that will be frequently referenced in this module.

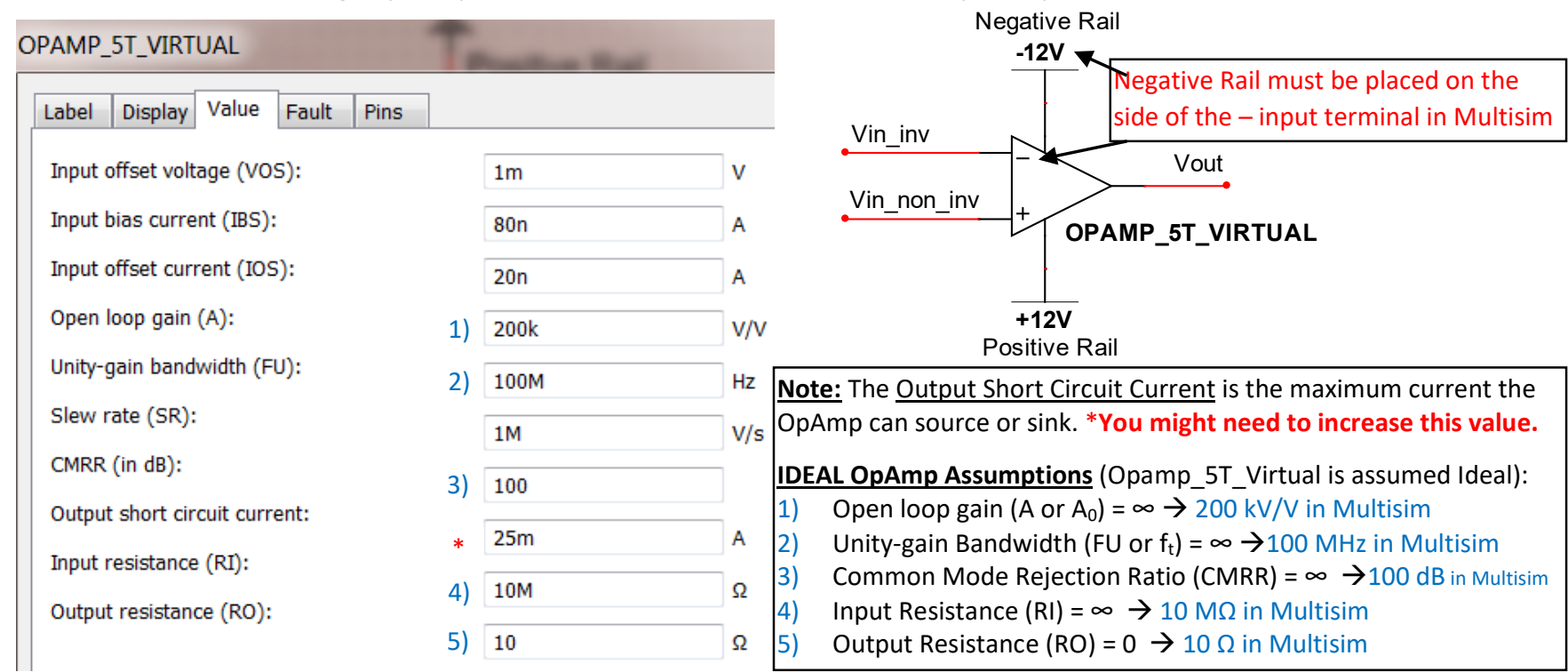

Figure 7.3: 5-Terminal Virtual OpAmp Internal Parameters and method to connect power rails in Multisim.

The following link is a 94 page document that provides a good overview of OpAmps and includes many applications and useful OpAmp-based circuits. This document titled "HANDBOOK OF OPERATIONAL AMPLIFIER APPLICATIONS" had its latest revision in September 2016. http://www.ti.com/lit/an/sboa092b/sboa092b.pdf 
One thing that is not addressed in that TI Handbook is active filtering. Module 4 described passive filtering with RLC circuits, but for many applications active filtering with OpAmps is preferred. The following two links to $\mathrm{TI}$ documents provide good information about active filtering.

- SLOA093: "Filter Design in 30 Seconds"

http://www.ti.com/lit/an/sloa093/sloa093.pdf

- SLOA096: "More Filter Design on a Budget" http://www.ti.com/lit/an/sloa096/sloa096.pdf

I would be remiss if I didn't also mention "The Art of Electronics" by Horowitz and Hill because many consider it the ultimate source for anything involving analog electronics. Unfortunately, there is no eBook on this one, but having this document on your shelf will make you feel like a real Electrical or Computer Engineer and give you a nice reference to have on hand in case you need an additional resource.

\section{Section 7.1 - OpAmps Used as Comparators}

A comparator is a device that compares two input pins and sends the output to one of the rails depending on which output is higher. Ideally, an IC specially designed to operate as a comparator (like the LM293) would be used for this type of application, but people often will simply use an OpAmp in "open loop" mode (i.e. without feedback). The following TI document explains the reasons for not using OpAmps as comparators and also elaborates on the differences between them: SLOA067 - Op Amp and Comparators - Don't Confuse Them!

With that said, if an OpAmp is used as a comparator no feedback is included to limit the gain of the OpAmp (as will be done when designing amplifiers in Section 7.2). Since the gain (Vout/Vin) is not limited it will be equal to the "Open Loop Gain, $A_{0}$ " (or internal gain of the OpAmp), which is nearly infinite. Since the Vout is limited by the rail voltages the output of a comparator is always equal to one of the rail voltages (+Vcc, $-\mathrm{Vcc}$, or ground). Figure 7.4 shows a dual supply comparator circuit (Left) and a single supply comparator circuit (Right) drawn with the positive and negative supplies connected to the locations that will allow the circuit to be correctly simulated in Multisim. These "reversed positive and negative rail connections" will be done in all circuits in the remainder of this module, but remember that it is the opposite of how it will normally be shown in technical documents.

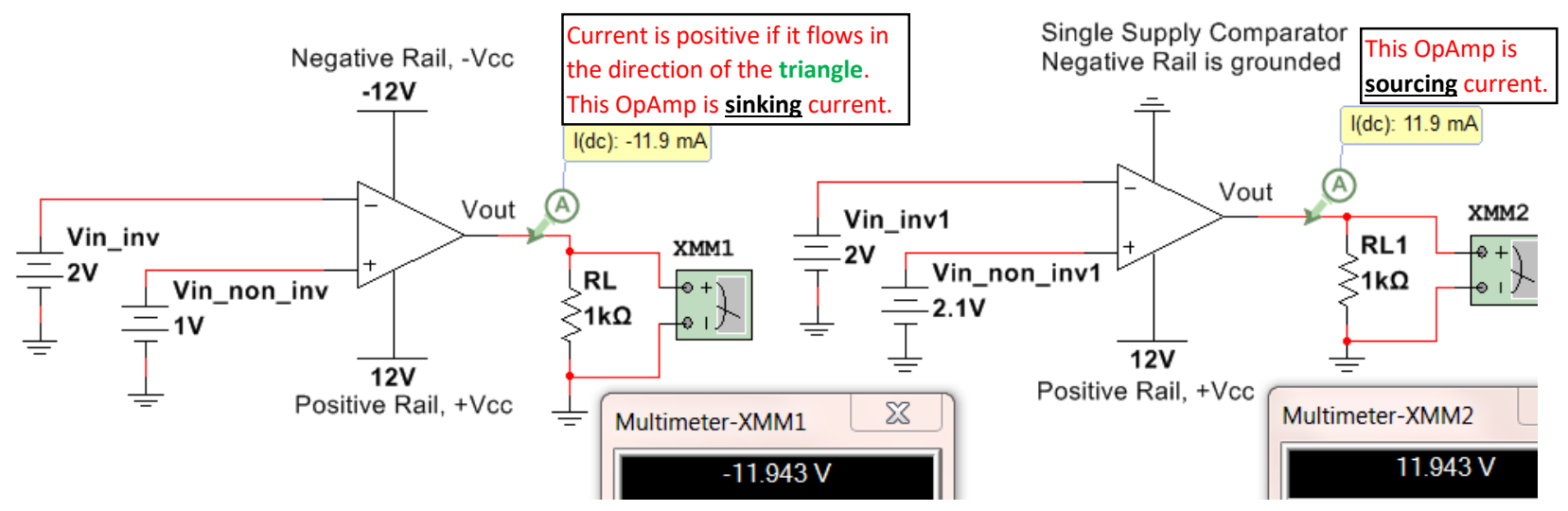

Figure 7.4: Dual Supply Comparator (Left), Single Supply Comparator (Right)

- If Vin_non_inv $>$ Vin_inv then Vout $\approx+$ Vcc. $\quad$ or $\quad V(+)>V(-)$ then Vout $\approx+$ Vcc

- If Vin_inv > Vin_non_inv then Vout $\approx-$ Vcc for the dual supply circuit or $\underline{0 V}$ for the single supply circuit.

Vout is assumed to go all the way to the rail voltage in open loop configurations, but there is often a signal swing limit that will prevent the OpAmp from going all the way to the rail. In Figure 7.4 Vout only goes to $11.943 \mathrm{~V}$ instead of $12 \mathrm{~V}$. Special OpAmps designed to go "rail to rail" are often needed if Vout needs to go to Vcc. Once Vout is determined from the two bullets above the current through the load $\left(R_{L}\right)$ can be calculated as $I_{R L}=$ Vout/RL. 
- If Vout is $>0$ then current is sourced OUT OF the OpAmp and down through the load.

- If Vout is $<0$ then current is sinked INTO the OpAmp and up through the load.

Example 7.1) Determine Vout and the magnitude and direction of the current across the load resistor in the following circuit before (Left circuit) and after the switch is closed (right circuit) if Vin $=5 \mathrm{~V}$. Does the OpAmp source or sink current for each case? The simulated results are shown in the Figure below.
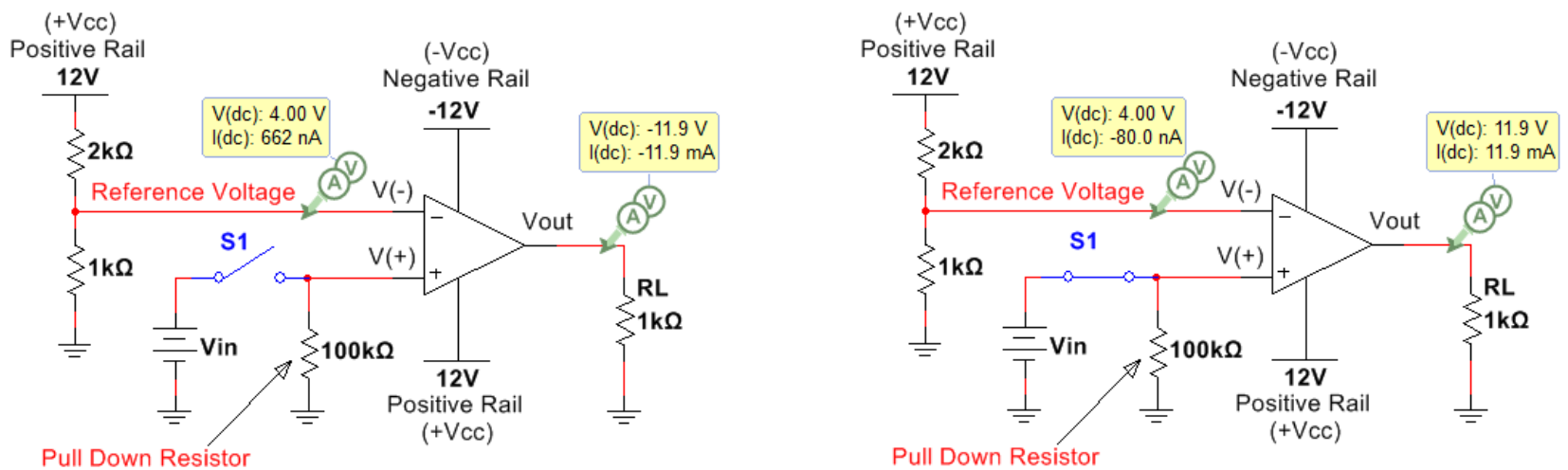

Figure 7.5: Circuit before (Left) and after (Right) Switch, S1 is closed if Vin = 5 V.

Before solving this problem some explanations are needed. When designing a comparator circuit (either with an OpAmp or with a comparator IC) one of the input pins (either $V(+)$ or $V(-)$ ) is often set to a fixed reference voltage. Since almost no current flows into either of the input pins of an OpAmp or a comparator IC due to the near-infinite input resistance a voltage divider can be used to set the reference voltage. In Figure 7.5, for both the left and right circuits, the reference voltage (Vref) is set using the following voltage divider rule (VDR) equation:

- $\operatorname{Vref}=12 \cdot 1 \mathrm{k} \Omega /(1 \mathrm{k} \Omega+2 \mathrm{k} \Omega)=\underline{\mathbf{4} \mathrm{V}}$

Vref is tied to $V(-)$ in this circuit, but it could just have easily been connected to $V(+)$ if the situation called for it. This is a very practical circuit that is used extensively when integrating sensors in circuitry. The "Vin" voltage in Figure 7.5 could be the voltage from a sensor and the design calls for Vout to go to $12 \mathrm{~V}$ when Vin is greater than Vref and to $-12 \mathrm{~V}$ when Vin is less than Vref (or if the switch isn't closed). With that said it is time to solve the problem.

\section{Before the Switch is closed}

- The pulldown resistor is necessary so that before the switch is closed $\mathbf{V}(+)$ is tied to ground (0V). Without the pulldown resistor $\mathrm{V}(+)$ would be connected to an open circuit and would be "floating" at an unknown voltage. A large value is usually used for a pullup or pulldown resistor so there is not very much current wasted.

- As previously stated $V(-)=V$ ref $=4 \mathrm{~V}$ from the VDR calculation.

- Since $4 \mathrm{~V}>0 \mathrm{~V} \rightarrow \mathrm{V}(-)>\mathrm{V}(+) \rightarrow$ Vout $=\mathbf{- 1 2} \mathbf{V}$

- $\quad$ The current flows up through the load $\rightarrow I_{R L}=(0--12) / 1000=\underline{12} \mathbf{~ m A ~ ( U p ) ~}$

- The OpAmp is sinking current since the current flows into it. The negative supply is supplying all of the power to this circuit before the switch is closed.

\section{After the Switch is closed}

- $\operatorname{Vin}(5 \mathrm{~V})$ is connected to the $100 \mathrm{k} \Omega$ pulldown resistor making $\underline{\mathbf{V}(+)} \mathbf{= 5 \mathbf { V }}$. 
- As previously stated $\mathrm{V}(-)=\mathrm{Vref}=4 \mathrm{~V}$ from the VDR calculation.

- Since $5 \mathrm{~V}>4 \mathrm{~V} \rightarrow \mathrm{V}(+)<\mathrm{V}(-) \rightarrow$ Vout $=+\mathbf{1 2} \mathrm{V}$

- The current flows down through the load $\rightarrow I_{R L}=(12-0) / 1000=\underline{\mathbf{1 2}} \mathbf{~ m A ~ ( D o w n ) ~}$

- The OpAmp is sourcing current since the current flows out of it. The positive supply is supplying all of the power to this circuit after the switch is closed.

If using an actual comparator IC (like the previously mentioned LM293) was used the first step to simulating it in Multisim would be to search for the part. Figure 7.6 shows how to search for it. Select "All Groups: and type in LM293 in the component field. In this case, 4 options including LM293 at the beginning of the name appear and all are surface mount ICs, but they will have close to the same properties as the through-hole DIP version. The LM293 is an 8-pin Dual comparator which means there are two comparators in the IC.

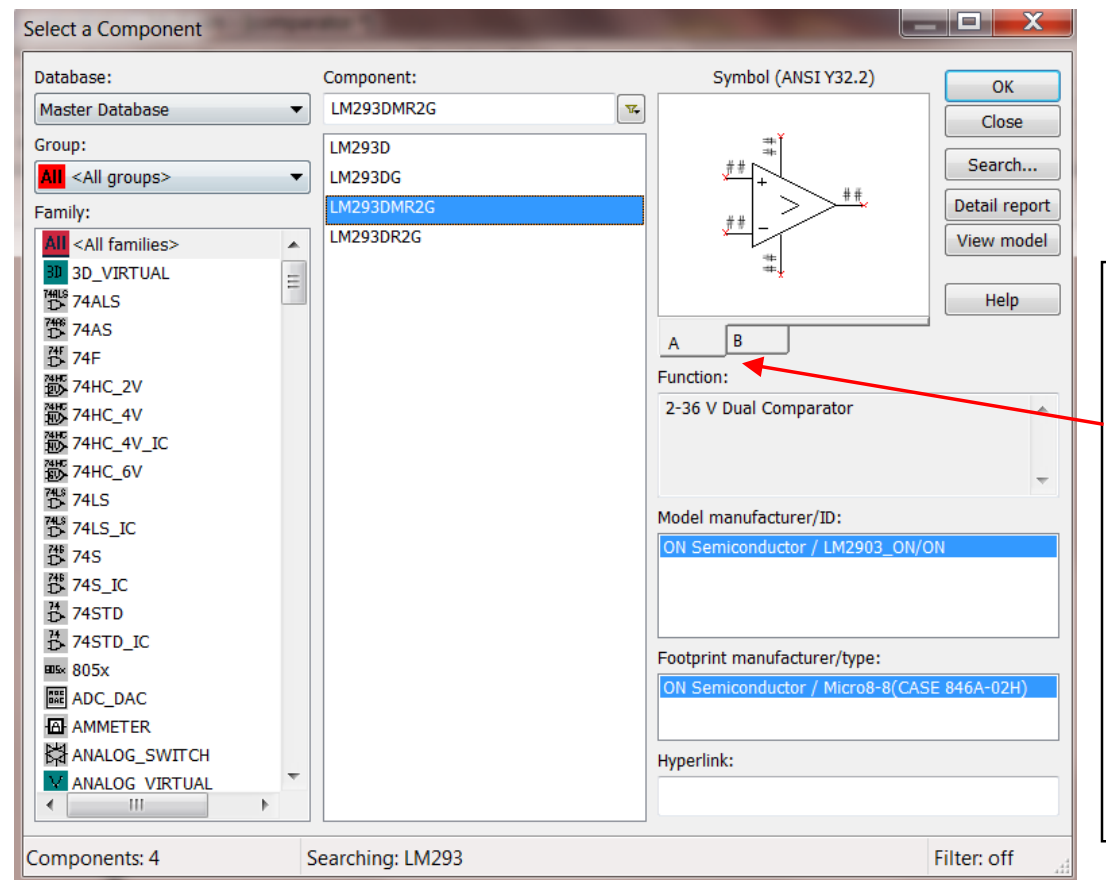

Two Comparators can be selected (A, B). The pin numbers will be different depending on which one is selected. In Figure 7.7 both A and $B$ are selected and all pin numbers are shown in Multisim. Since the 2 comparators on the Dual IC have a common ground and Vcc only one has to be connected to $\mathrm{Vcc}$ and ground and the other two show a red $\mathrm{x}$.

Figure 7.6: Searching for parts in Multisim - LM293, 8-pin Dual Comparator selected
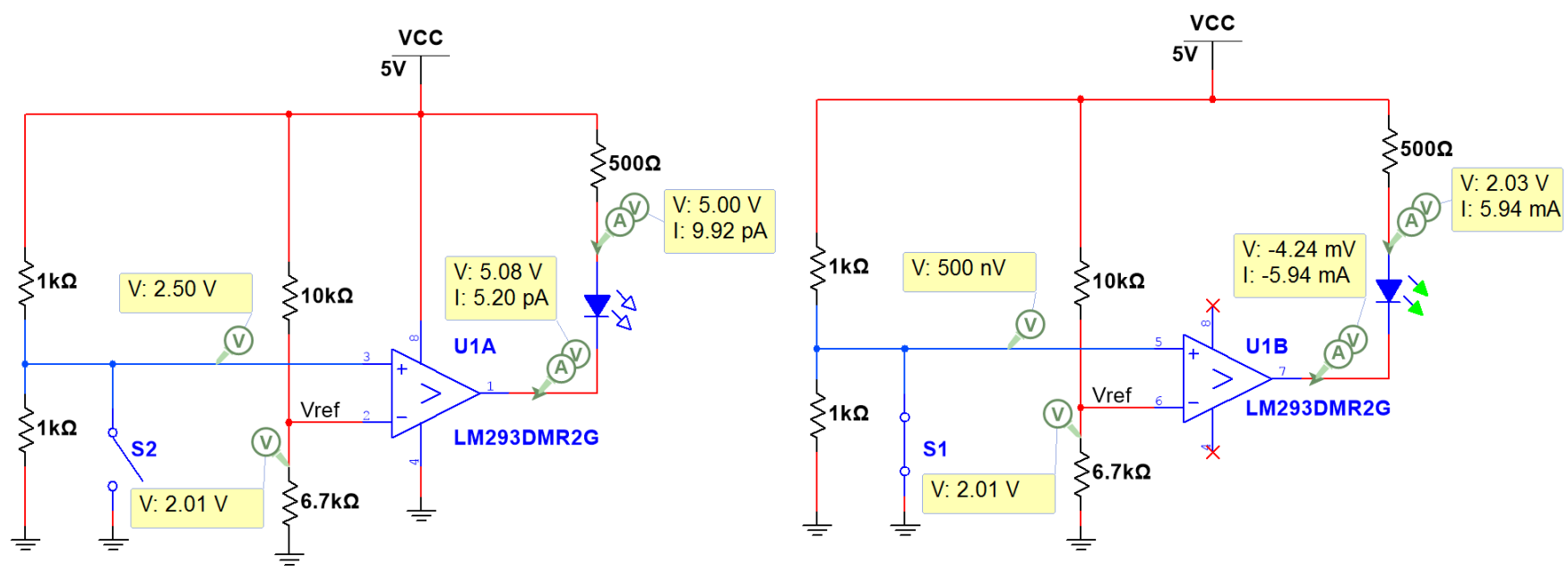

Figure 7.7: Circuit using the LM293 showing that the output goes low and sinks current when $\underline{\mathrm{IN}->\mathrm{IN+}}$ 
The circuit in Figure 7.7 shows that the LM293 operates in an active low configuration, where it turns an LED on by sinking current through it when IN- > IN+ (Note: IN- is labeled Vref on the schematic). The LM293 has a high impedance (or Open Collector) output so a pullup resistor has to be used for it to work the way it is was intended. In Figure 7.7 the pullup resistor is put in series with an LED so it will turn on an LED when the output goes low. When IN+ > IN- it doesn't source current like a normal OpAmp operates (as shown in Figure 7.5). Instead, it sends the output to Vcc so that no current flows between $\mathrm{Vcc}$ and the pullup resistor because there is no voltage difference $\rightarrow$ Recall from the Davis DC Circuits book: $I_{R}=\Delta V / R=(V c c-V c c) / R=0$

\section{Section 7.2 - OpAmps used as Amplifiers}

For this section the OPAMP_5T_VIRTUAL part in Multisim will also be used for all circuits except the one in Figure 7.9 and Figure 7.11. Those circuits are built using the LM324 that was described in Figure 7.1.

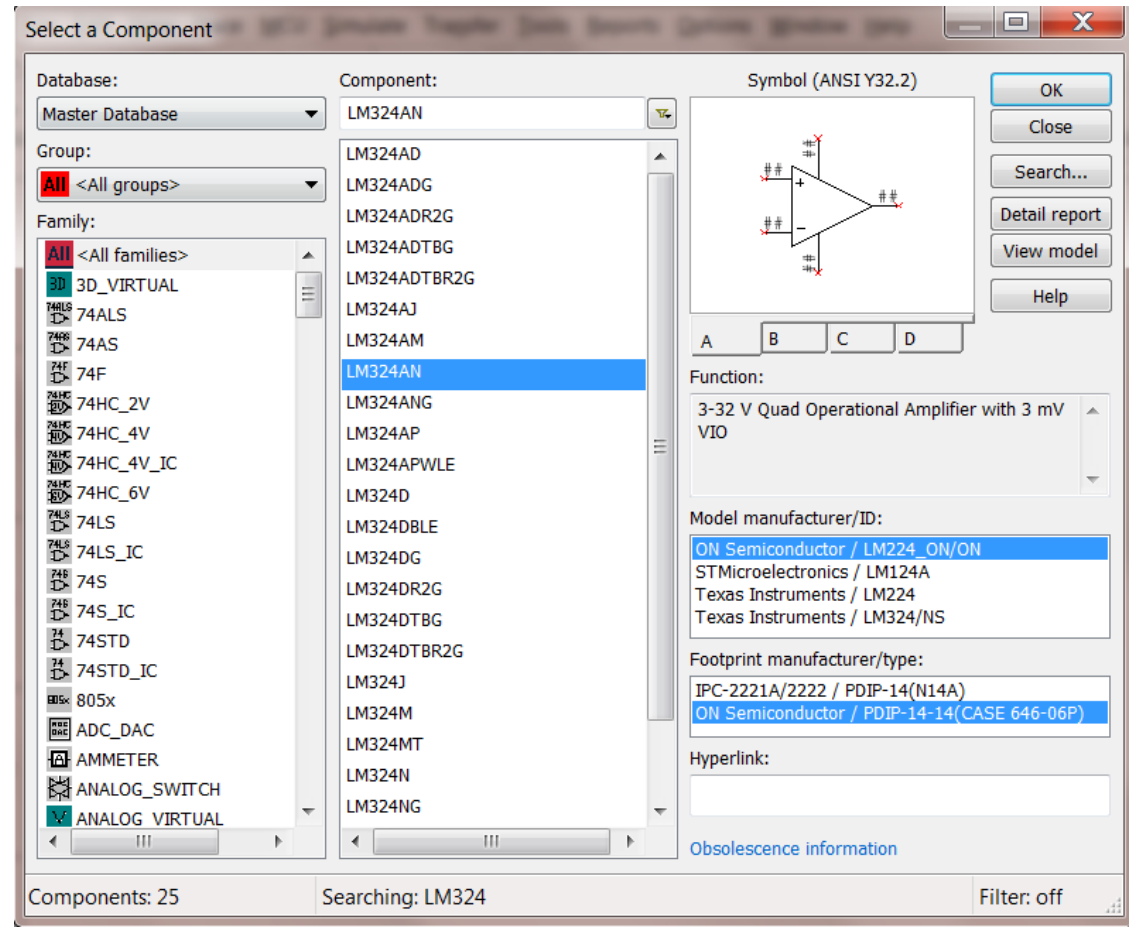

Of the 25 LM324 options, a DIP version
from Fairchild Semiconductor (referred
to in Figure 7.1) will be selected
(LM324AN). Note: An ON
Semiconductor part is selected instead
because they acquired Fairchild
semiconductor in 2015. PDIP stands for
"Plastic Dual Inline Package" and is one
of the most popular IC types for
through-hole applications. Note: DIP
packages will have higher power
ratings than surface mount parts. The
LM224 is selected in the "Model
manufacturer/ID", because this is in
the same family as the LM324. Notice,
there are 4 OpAmps (A, B, C, D) that
can be selected. In Figure 7.9 an
example is shown that uses all 4
OpAmps in a circuit.

\section{Figure 7.8: Searching for parts in Multisim - LM324 14-pin Quad DIP OpAmp selected}

The circuits in Figures 7.9 to 7.12 include four OpAmps and is used to amplify a signal at four different gains and turn on LEDs. Figure 7.9 shows has a DC input voltage and shows how all 14 of the LM324 quad OpAmp would be implemented for this circuit and Figure 7.10 shows the results if the LM 324 is replaced with four virtual OpAmps (OpAmp_5T_Virtual). The green LED has an adjustable current threshold. By double clicking on the LED in Multisim the threshold can be changed. The $5 \mathrm{~mA}$ default is used in all of the figures in this eBook. For the circuits in Figure 7.9 and Figure 7.10 with a DC input of $120 \mathrm{mV}$ all OpAmps source enough current to turn on the LEDs except the OpAmp connected to LED4. The $470 \Omega$ resistor is used to limit the current through the LEDs to around $20 \mathrm{~mA}$, which is a common current rating for many types of LEDs. When the input is a DC voltage the results are nearly identical for the LM324 (igure 7.9) and the virtual OpAmp (Figure 7.10). The only significant difference can be seen in the output of the top left OpAmp that is connected to LED1. It has a gain large enough to saturate the OpAmp. Saturating the OpAmp (also commonly referred to as clipping) occurs when your output is limited by the rail voltage. The Virtual OpAmp allows Vout to go very close to the positive $12 \mathrm{~V}$ rail, but the LM324 limits the voltage to 10.3 Volts in this example. 


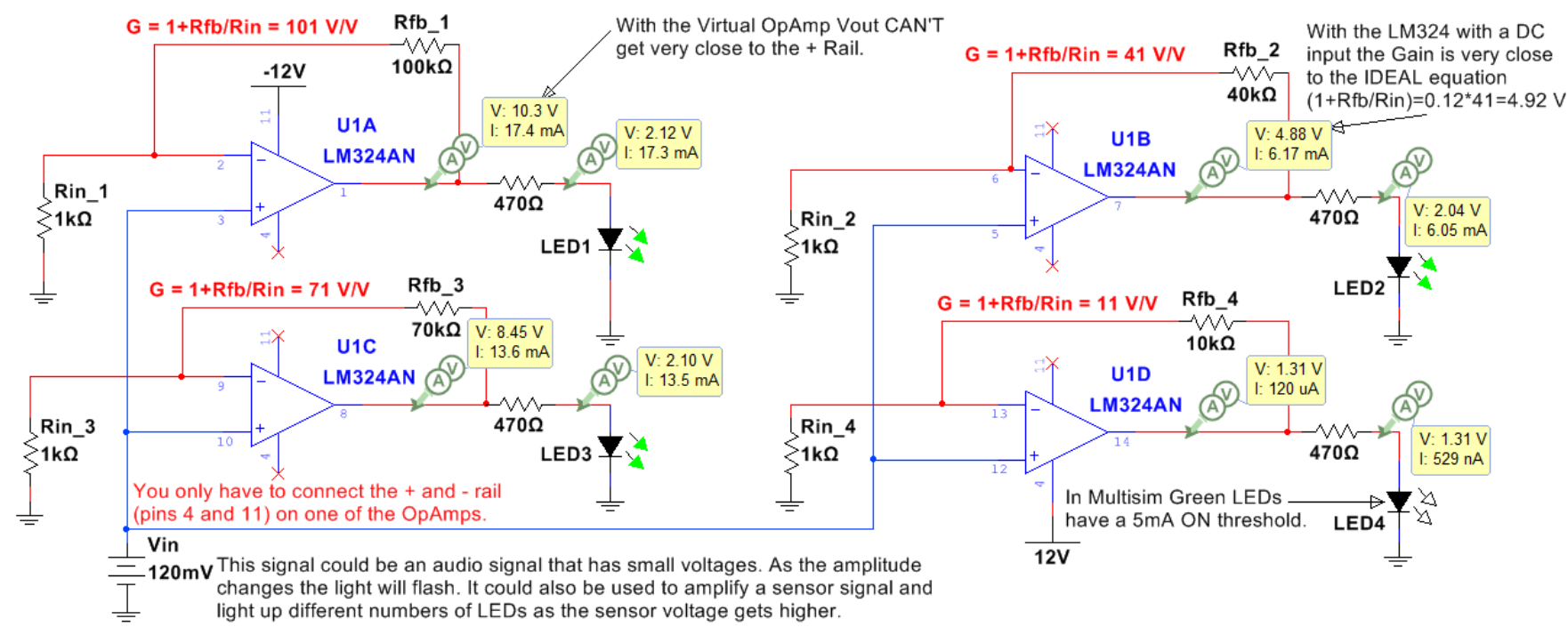

Figure 7.9: Non-Inverting Configuration Example that uses all 4 of the LM324 OpAmps with a DC input.

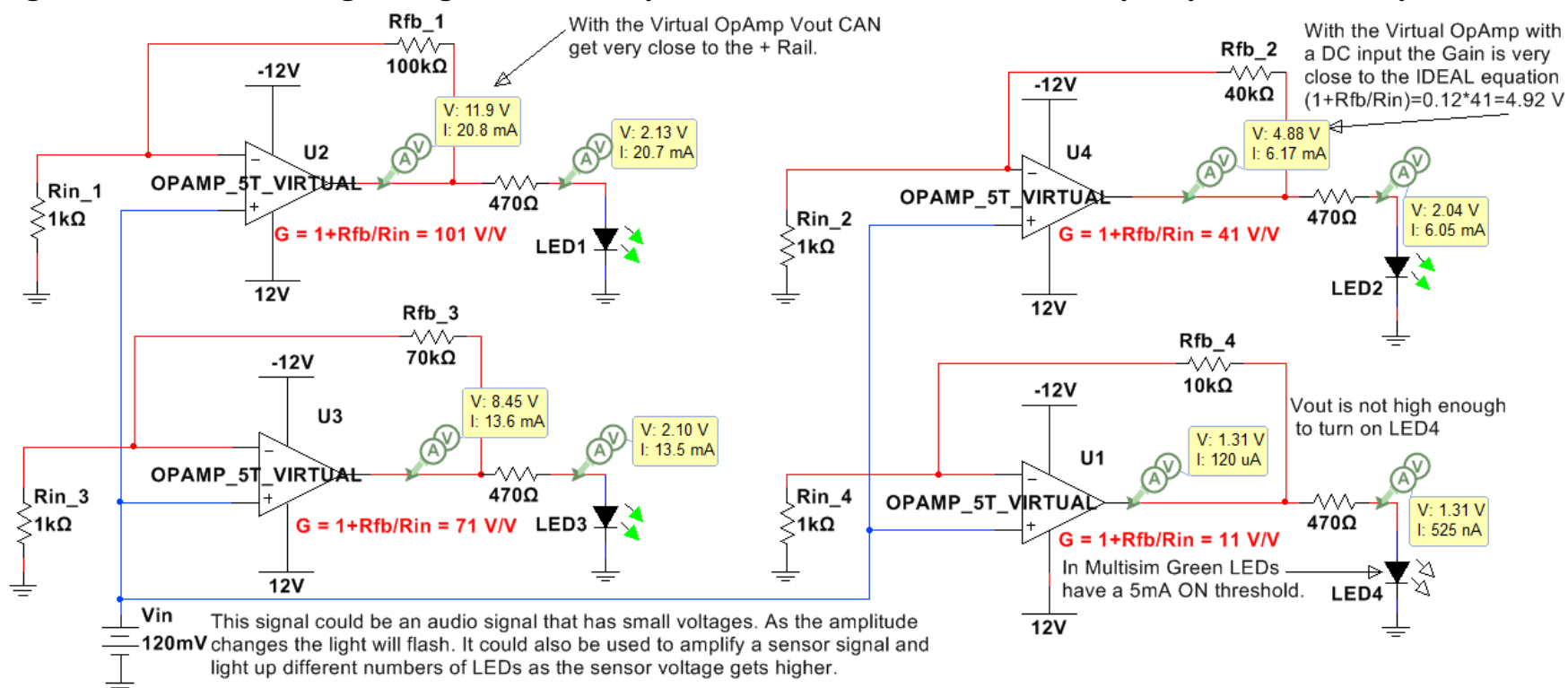

Figure 7.10: The circuit in Figure 7.9 with the OpAmps replaced with the Virtual 5 Terminal OpAmp.

For DC input signals, the closed loop gain ( $G=$ Vout/Vin) is usually very close to the Ideal OpAmp_gain

(Equations 7.2 and 7.3 for the inverting and non-inverting OpAmp configurations respectively) for all types of OpAmps because the open loop gain $\left(A_{0}\right)$ is always an extremely large value (e.g. 200,000 V/V for the Virtual OpAmp in Figure 7.3), but in reality $A_{0}$ and $G$ are reduced as the frequency increases once the frequency reaches the OpAmps internal $3 \mathrm{~dB}$ frequency (which usually occurs at low frequencies). For example, the $3 \mathrm{~dB}$ internal frequency for the LM324 is approximately $30 \mathrm{~Hz}$ when $\mathrm{Vcc}=15 \mathrm{~V}$ and $\sim 80 \mathrm{~Hz}$ when $\mathrm{Vcc}=30 \mathrm{~V}$ as shown in Figure 5 of the LM324 datasheet. The ideal closed loop gain equations are modified for the situation where $A_{0}$ is NOT assumed to be infinity and are shown in Equation 7.4. When the signal is a high frequency sinusoid then the Vout is greatly reduced in the LM324 (Figure 7.11), but not in the Virtual OpAmp (Figure 7.12) because the Virtual OpAmp assumes $A_{0}$ remains at 200,000 V/V for all frequencies. 


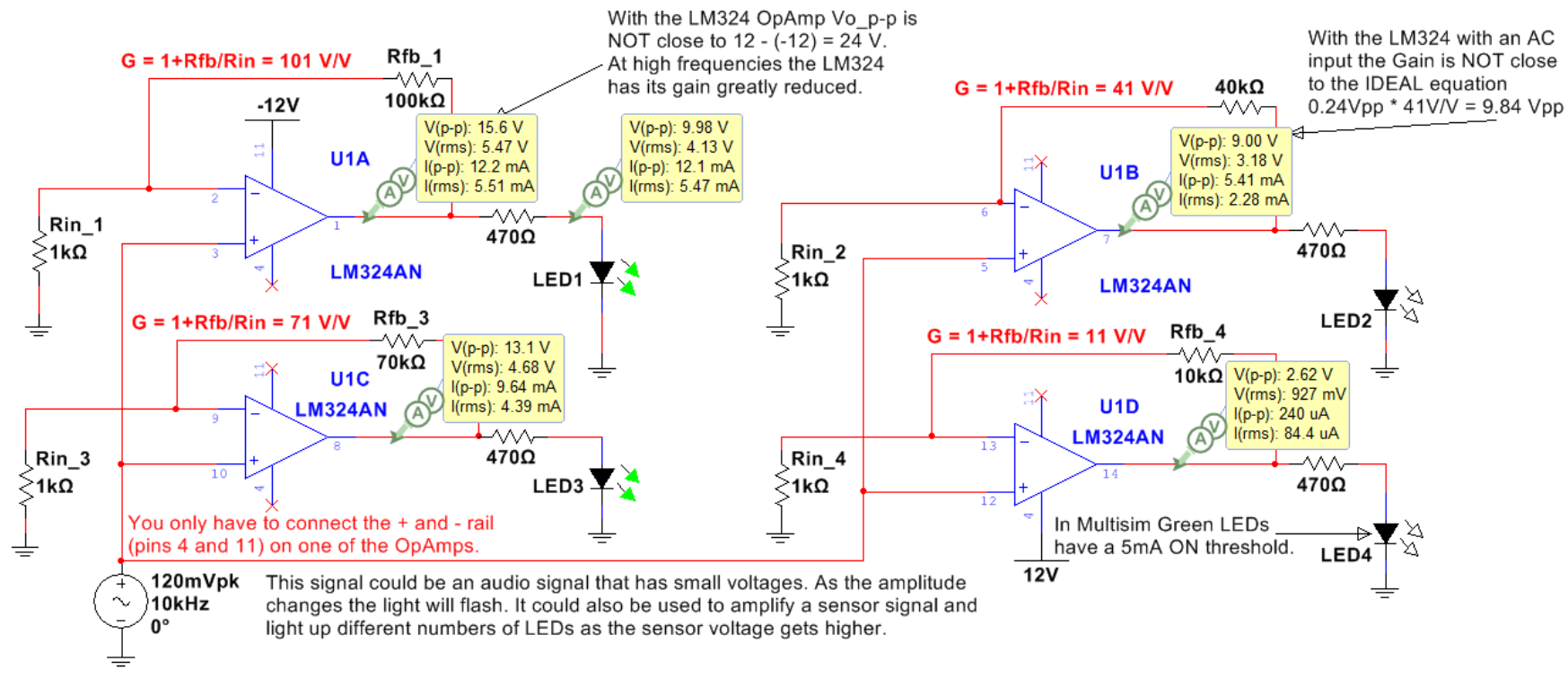

Figure 7.11: Example circuit that uses all 4 of the LM324 OpAmps with an $10 \mathrm{kHz}$ AC input.

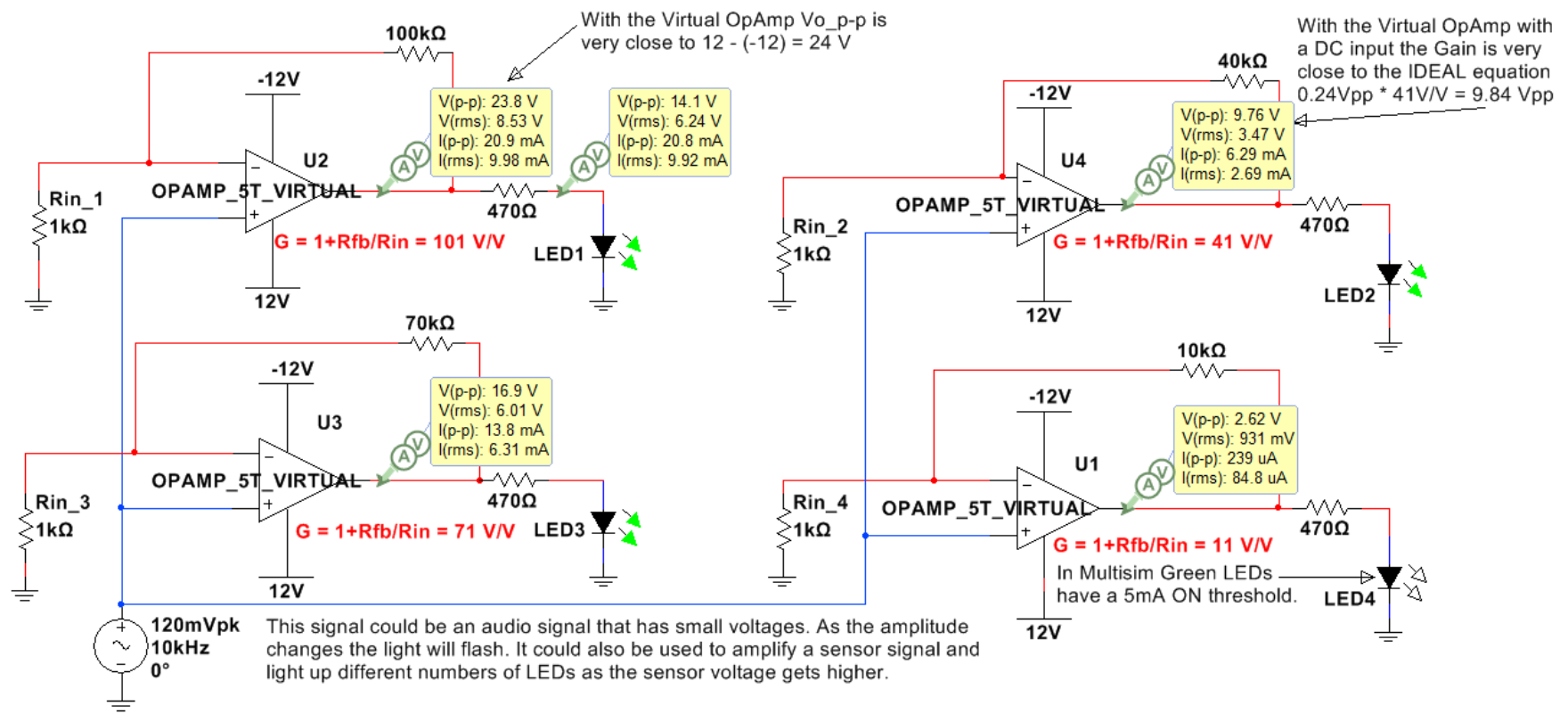

Figure 7.12: The circuit in Figure 7.11 with the OpAmps replaced with the Virtual 5 Terminal OpAmp.

Important OpAmp terms that have been previously mentioned are described in more detail in the bullets below along with the inverting and non-inverting gain equations. Sections $\underline{7.2 .1}$ and $\underline{7.2 .2}$ show example circuits.

- Closed loop voltage gain $(G)$ is equal to Vout/Vin when a feedback resistor $\left(R_{f b}\right)$ is connected from the output pin of the OpAmp to the inverting (-) terminal of the OpAmp as shown in the circuits in Figure 7.9 to 7.12. If $R_{\mathrm{fb}}$ is replaced with a wire (or $R_{\mathrm{fb}}=0 \Omega$ ) the gain is equal to $1 \mathrm{~V} / \mathrm{V}$ for the non-inverting configuration and $0 \mathrm{~V} / \mathrm{V}$ for the inverting configuration.

- Open loop voltage gain $\left(A_{0}\right)$ is the internal gain of the OpAmp. $A_{0}$ is assumed to be equal to infinity at low frequencies and decrease by approximately $20 \mathrm{~dB} / \mathrm{dec}$ as frequency increases. $A_{0}$ would be the gain of the OpAmp if no feedback resistor is included and the OpAmp operates in the open loop mode as a 
comparator as shown in Figures 7.4 and 7.5. Ao will always be large enough to send the output to one of the rail voltages if no feedback $\left(R_{\mathrm{fb}}\right)$ is included in the OpAmp.

- Ideal Gain equations are simplified equations for closed loop gain and have the following assumptions:

1) Open loop gain $\left(A_{0}\right)=\infty$

2) Unity-gain Bandwidth (FU or $\left.\mathrm{f}_{\mathrm{t}}\right)=\infty$

3) Common Mode Rejection Ratio (CMRR) $=\infty$

4) Input Resistance (RI) $=\infty$

5) Output Resistance (RO) $=0$

- As frequency increases the assumptions 1) and 2) of the ideal model are not valid and cause the open loop gain $\left(A_{0}\right)$ to become small enough that the ideal gain equations become increasingly inaccurate. This results in the need for a new set of gain equations where $A_{0}$ is assumed to be finite. If the input frequency is beyond the internal $3 \mathrm{~dB}$ frequency of the OpAmp $\left(f_{3 \mathrm{db}}\right)$ a good approximation of $A_{0}$ can be found from Equation 7.1, where $f_{\text {in }}$ is the input frequency and $f_{t}$ is the OpAmps Unity Gain Bandwidth.

[7.1]

$$
A_{0} \cong \frac{f_{t}}{f_{\text {in }}}
$$

* This is a good approximation when $f_{\text {in }}>f_{3 d B}$

- Inverting Configuration: If the input voltage is connected the in input resistance $\left(R_{\text {in }}\right)$ that is connected to the inverting (-) terminal of the OpAmp it is called the "inverting configuration". An example with a DC input is shown in Figure 7.13 and an example with an AC signal is shown in Figure 7.14. The closed loop gain equations for an inverting OpAmp are shown in 7.2 and 7.3.

$[7.2]$

$$
G_{\text {ideal }}=\frac{v_{\text {out }}}{v_{\text {in }}} \cong \frac{-R_{f b}}{R_{\text {in }}}
$$

- Non-Inverting Configuration: If the input voltage is connected to the in non-inverting input terminal (+) of the OpAmp it is called the "non-inverting configuration". An example with a DC input is shown in Figure 7.15 and an example with an AC signal is shown in Figure 7.16.

$$
G_{\text {ideal }}=\frac{v_{\text {out }}}{v_{\text {in }}} \cong 1+\frac{R_{f b}}{R_{\text {in }}}
$$

- Finite $A_{0}$ gain OpAmp equation: If $A_{0}$ cannot be assumed to be infinity (usually because the input frequency it too high) then equations 7.2 and 7.3 can be corrected using Equation 7.4.

$$
G=\frac{v_{\text {out }}}{v_{\text {in }}} \cong \frac{G_{\text {ideal }}}{1+\frac{1+\frac{R_{f b}}{R_{\text {in }}}}{A_{0}}}
$$

* Note: When $A_{0}$ is infinity then $G=G_{\text {ideal. }}$

\section{Section 7.2.1 - Inverting OpAmp Configuration}

The inverting OpAmp configuration is shown in Figure 7.13 (DC input) and Figure 7.14 (AC input). 


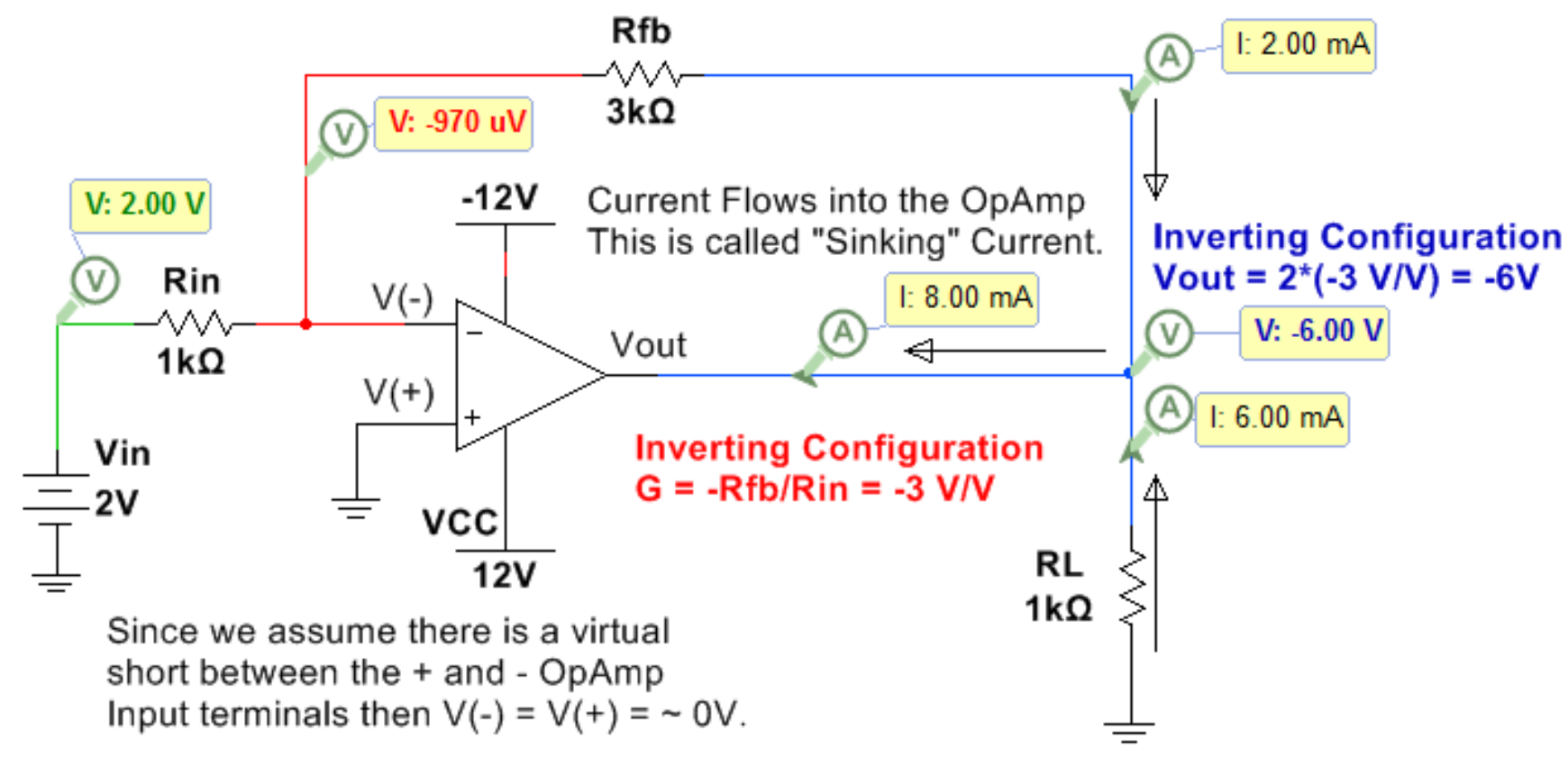

Figure 7.13: Inverting Configuration with a DC input.

In the circuit below the instantaneous voltage values (top numbers) are also shown so you can see that at that moment in time the circuit behaves exactly like the DC circuit in Figure 7.13 (Vout $=-.994 \mathrm{~V} \cdot(-3 \mathrm{~V} / \mathrm{V})=2.98 \mathrm{~V})$.

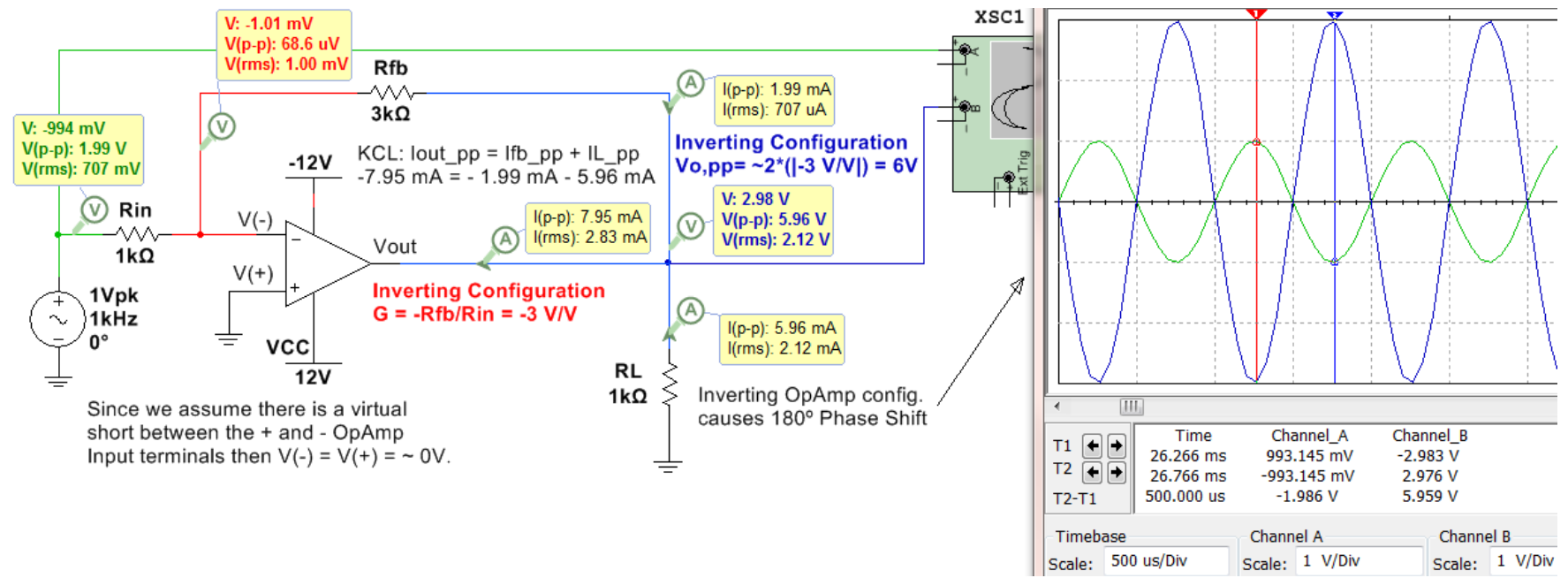

Figure 7.14: Inverting Configuration with an $A C$ input.

Since the virtual OpAmp in Figure 7.14 has a fixed $A_{0}$ of $200,000 \mathrm{~V} / \mathrm{V}$ regardless of the input frequency the ideal gain in Equation 7.2 matched closely with the Multisim measurements. If an actual OpAmp were used (like the LM324) the gain would be reduced due to the increase in frequency that results in a finite $A_{0}$. For this same reason, Multisim also matches the Non-inverting OpAmp configuration circuit with an AC input in Figure 7.16. 
Section 7.2.2 - Non-Inverting OpAmp Configuration

The non-inverting OpAmp configuration is shown in Figure 7.15 (DC input) and Figure 7.16 (AC input).

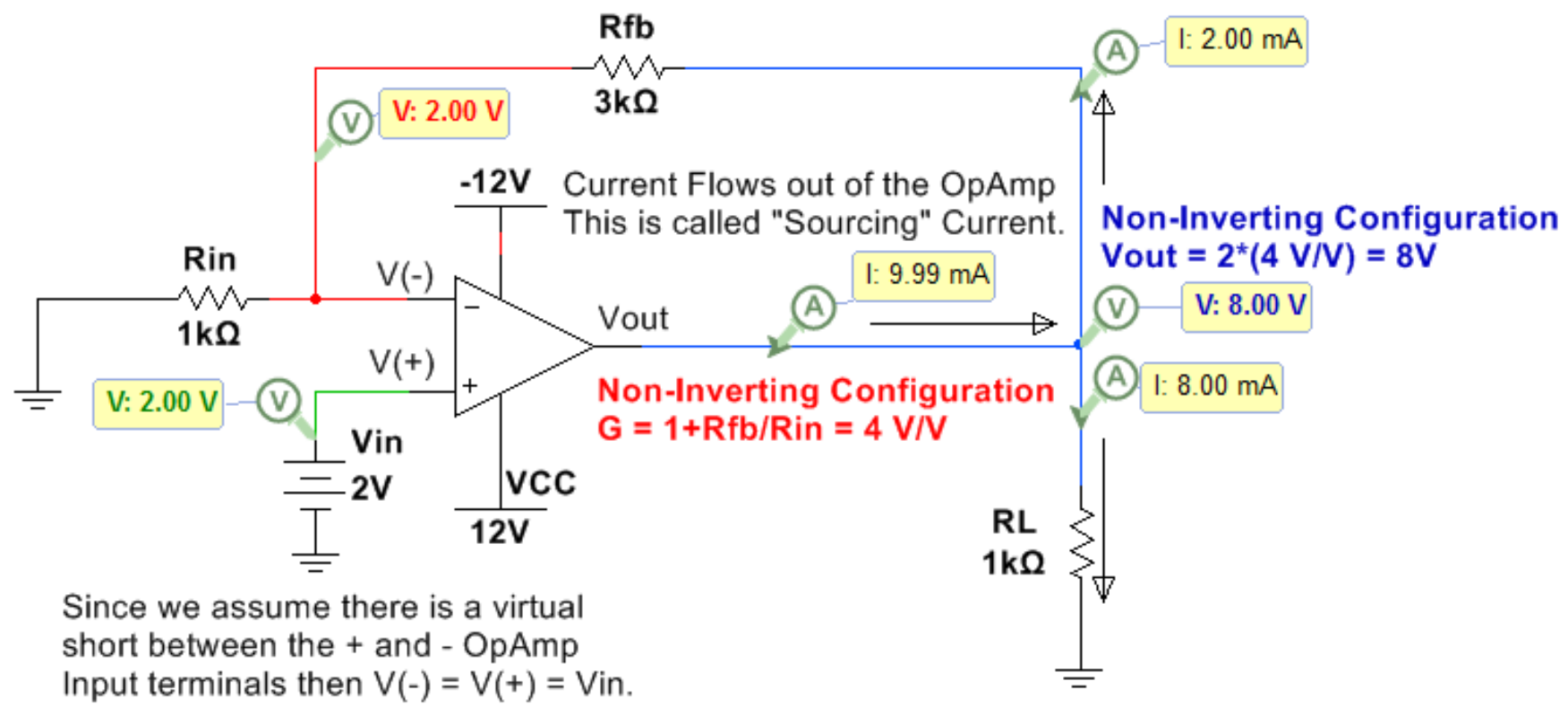

Figure 7.15: Non-Inverting Configuration with a DC input.

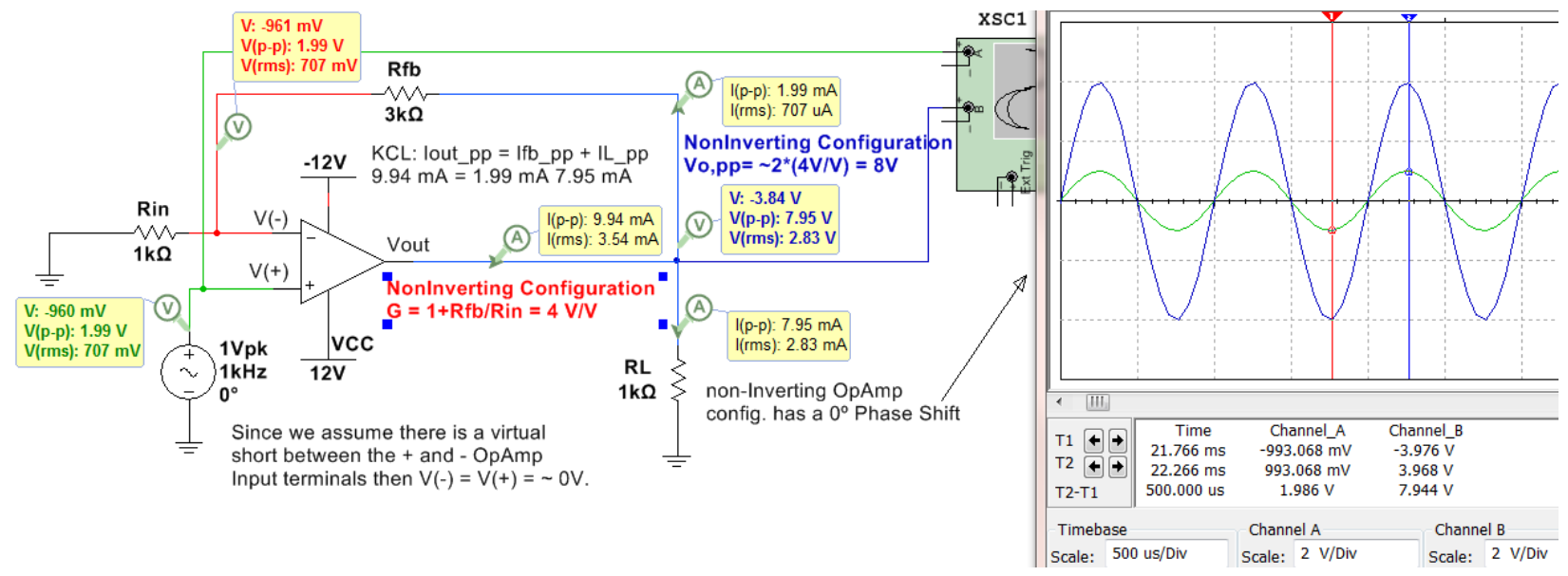

Figure 7.16: Non-Inverting Configuration with an AC input. 


\section{Section 7.3 - Superposition with OpAmps}

With DC circuits the superposition principle was a good method for solving circuits with multiple inputs and can also be applied to OpAmp circuits. Superposition is accomplished by breaking the overall problem with multiple inputs up into sub-problems where the circuit was solved "Due to Only" one input at a time and then the values are added together to get the result due to all inputs. The inputs not involved in the sub-problem are "zeroed out" as follows.

- Independent Voltage Sources are replaced with short circuits.

- Independent Current Sources are replaced with open circuits.

Using the equations in section 7.2 and the superposition method more complicated OpAmp circuits can be solved. The example in Figure 7.17 shows a "Differential Amplifier" configuration where Rin and Rfb from section 7.2 is replaced with R1 and R2. Note: In OpAmp technical documents $R_{\mathrm{fb}}$ is commonly referred to as R2 and $R_{\text {in }}$ is commonly referred to as R1. A voltage divider with R3 and R4 is used on the + OpAmp input such that $\mathrm{V}(+)=\mathrm{Vin} 2 \cdot \mathrm{R} 4 /(\mathrm{R} 3+\mathrm{R} 4)$. Recall that this voltage divider is possible because we assume that NO Current flows into either input terminal of the OpAmp due to the infinite input impedance assumption.

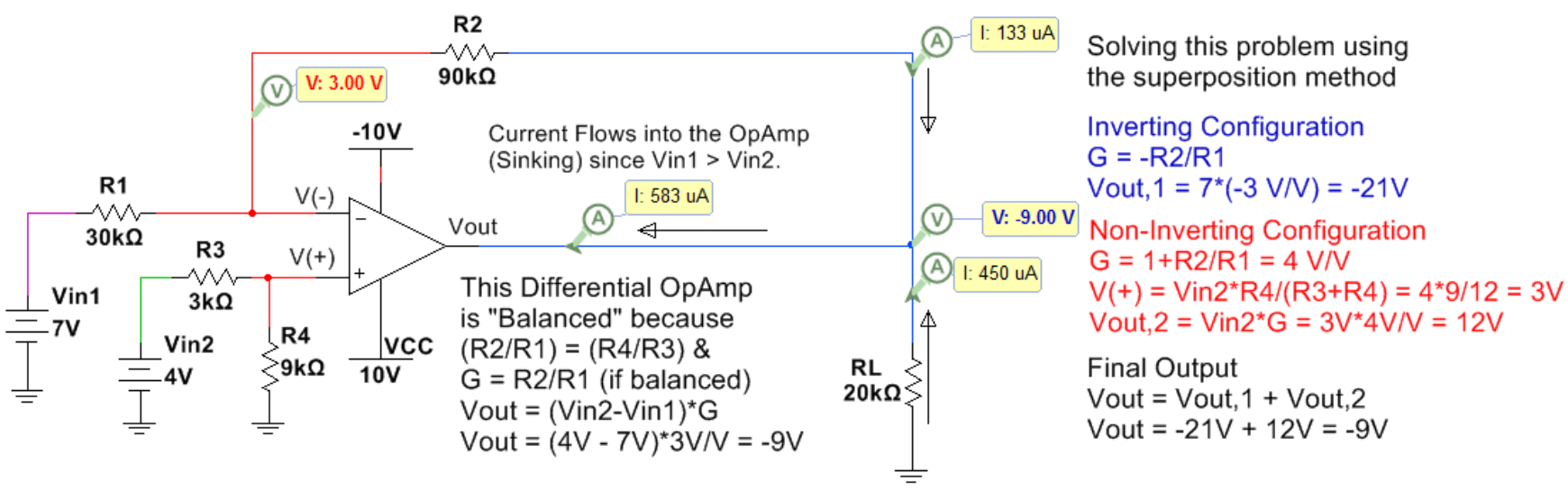

Figure 7.17: Differential OpAmp Configuration.

In Figure 7.18, a summing amplifier is simulated and solved using the superposition method. Two additional OpAmp circuits are shown in the Appendix: $4^{\text {th }}$ order Butterworth Low Pass Filter in Figure A.4 and an instrumentation amplifier in Figure A.5.

The equation for a summing OpAmp is:

Vout $=-\operatorname{Rfb} / \operatorname{Rin} 1{ }^{*}$ Vin1 - Rfb/Rin2*Vin2 - Rfb/Rin3*Vin3 $=3(-1)+4(-1)+5(-1)=-12 \mathrm{~V}$

Note: A weighted summer can be created by modifying the resistor values.

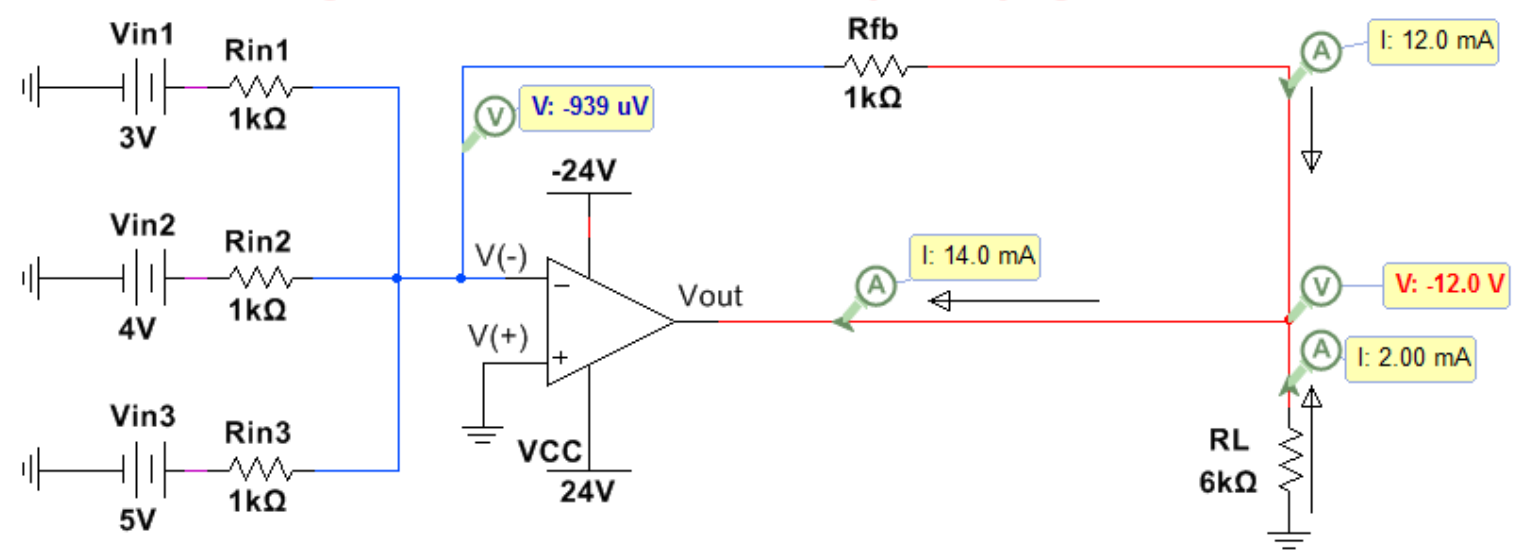

Figure 7.18: Summing OpAmp Configuration. 


\section{References and Links}

The following references and links provide supporting information for this module. All references in this module are either cited within the module within brackets or URL links are either embedded in hyperlinks or fully listed.

\section{References and Links not listed in other Modules}

[1] DC Circuits, Chad Davis: https://shareok.org/handle/11244/52245

[2] http://www.allaboutcircuits.com/textbook/semiconductors/

[3] Electronics Overview: http://www.allaboutcircuits.com/textbook/semiconductors/

[4] Multisim: http://www.ni.com/multisim/

\section{Module 1 - References and Links}

[5] AC and DC Background: http://www.allaboutcircuits.com/news/why-is-the-us-standard-60-hz/

[6] Electrical Safety: http://www.allaboutcircuits.com/textbook/direct-current/chpt-3/ohms-law-again/

[7] Frequencies of different musical notes: http://www.allaboutcircuits.com/textbook/alternatingcurrent/chpt-1/ac-waveforms/

[8] Sampling Information: http://www.dspguide.com/ch3/2.htm

[9] Square wave information: http://www.allaboutcircuits.com/textbook/alternating-current/chpt7/square-wave-signals/

[10] Square wave information: https://en.wikipedia.org/wiki/Square wave

[11] Triangle wave information: https://en.wikipedia.org/wiki/Triangle wave

\section{Module 3-References and Links}

[12] RLC Calculations: http://hyperphysics.phy-astr.gsu.edu/hbase/electric/powerac.html

[13] Rigol Oscilloscope Information: http://www.prc68.com/l/RigolDS1052E.shtml

[14] Error simulating inductors in MultiSim: https://forums.ni.com/t5/Multisim-and-Ultiboard/Offsetproblem-in-simulating-current-and-voltage-phase-relation/td-p/1194627

[15] Alternating Current eBook: http://www.allaboutcircuits.com/textbook/alternating-current/chpt-14/50ohm-cable/

[16] Electrical effects of the physical size of the transmission lines: http://www.allaboutcircuits.com/technical-articles/introduction-to-the-transmission-line/

[17] The following link shows a figure with more information about the electrical grid and includes different sources of electrical generation such as: wind, solar, hydro-electric, nuclear, and coal: https://commons.wikimedia.org/wiki/File:Electricity Grid Schematic English.svg

Module 4 - References and Links

[18] Filter Overview: http://www.allaboutcircuits.com/textbook/alternating-current/chpt-8/what-is-a-filter/

\section{Module 5 - References and Links}

[19] History of the transformer: https://en.wikipedia.org/wiki/War of Currents

[20] Transformer Basics: http://www.allaboutcircuits.com/textbook/alternating-current/chpt-9/mutualinductance-and-basic-operation/

[21] Transformer Video Lectures; http://www.allaboutcircuits.com/textbook/alternating-current/chpt9/mutual-inductance-and-basic-operation/

[22] Rectifiers: http://www.allaboutcircuits.com/textbook/semiconductors/chpt-3/rectifier-circuits/ 
[23] Faraday's Law: http://www.allaboutcircuits.com/textbook/alternating-current/chpt-9/mutualinductance-and-basic-operation/

[24] Internal Diagram of a Transformer:

https://en.wikipedia.org/wiki/Transformer\#/media/File:Transformer3d col3.svg

\section{Module 6 - References and Links}

[25] 1N914 switching diode datasheet: https://www.fairchildsemi.com/datasheets/1N/1N914.pdf

[26] 1N4000 series rectifier diode datasheet: https://www.fairchildsemi.com/datasheets/1N/1N4007.pdf

[27] Diodes Sparkfun tutorial: https://learn.sparkfun.com/tutorials/light-emitting-diodesleds? ga=1.259343308.774591033.1477860341

[28] Red LED ordering info: https://www.sparkfun.com/products/9590

[29] Green LED ordering info: https://www.sparkfun.com/products/9592

[30] Diode Overview: http://www.futureelectronics.com/en/diodes/diodes.aspx

[31] Diode Overview: http://www.allaboutcircuits.com/textbook/semiconductors/chpt-3/introduction-todiodes-and-rectifiers/

[32] BS170 MOSFET datasheet: http://www.onsemi.com/pub link/Collateral/BS170-D.PDF

\section{Module 7 - References and Links}

[33] LM324 OpAmp datasheet: https://www.fairchildsemi.com/datasheets/LM/LM324.pdf

[34] A Texas Instrument (TI) white paper SLOA011 titled Understanding Operational Amplifier Specifications: http://www.ti.com/lit/an/sloa011/sloa011.pdf

[35] Texas Instrument (TI) Handbook of Operational Amplifier Applications: http://www.ti.com/lit/an/sboa092b/sboa092b.pdf

[36] SLOA093: "Filter Design in 30 Seconds" http://www.ti.com/lit/an/sloa093/sloa093.pdf

[37] SLOA096: "More Filter Design on a Budget" http://www.ti.com/lit/an/sloa096/sloa096.pdf

[38] Art of Electronics: https://www.amazon.com/Art-Electronics-PaulHorowitz/dp/0521809266/ref=dp ob title bk

[39] SLOA067 - Op Amp and Comparators - Don't Confuse Them!: http://forums.parallax.com/discussion/download/96248\&d=1350228174 


\section{Appendix}

Figure A.1 shows the step response and the Bode magnitude plots for a series RL LPF with the same topology as shown in Figure 4.5 and a series RC HPF with the same topology as shown in Figure 4.6. The step response is the output voltage across the resistor if the input voltage is a $1 \mathrm{~V} \mathrm{DC}$ source that is suddenly applied with a switch. The LPF (RL Circuit with black curves in Figure A.1) shows that the input voltage is passed to the output since Vout at steady state is $1 \mathrm{~V}$. When studying $D C$ transients in the DC circuits eBook the equation for $\mathrm{v}_{\mathrm{C}}(\mathrm{t})$ and $\mathrm{v}_{\mathrm{R}}(\mathrm{t})$ was found to be: $v_{R}(\mathrm{t})=\operatorname{Vin}_{D C} \cdot\left(1-e^{-t / \tau}\right)$ and $v_{L}(\mathrm{t})=\operatorname{Vin}_{D C} \cdot\left(e^{-t / \tau}\right) \quad$ Click Here to Go Back to Module 4.

The HPF (RC Circuit with red curves in Figure A.1) shows that the input voltage is blocked and doesn't pass to the output since Vout at steady state is $1 \mathrm{~V}$. When studying DC transients in the DC circuits eBook the equation for $\mathrm{v}_{\mathrm{C}}(\mathrm{t})$ and $\mathrm{V}_{\mathrm{R}}(\mathrm{t})$ was found to be: $v_{C}(\mathrm{t})=\operatorname{Vin}_{D C} \cdot\left(1-e^{-t / \tau}\right)$ and $v_{R}(\mathrm{t})=\operatorname{Vin}_{D C} \cdot\left(e^{-t / \tau}\right)$
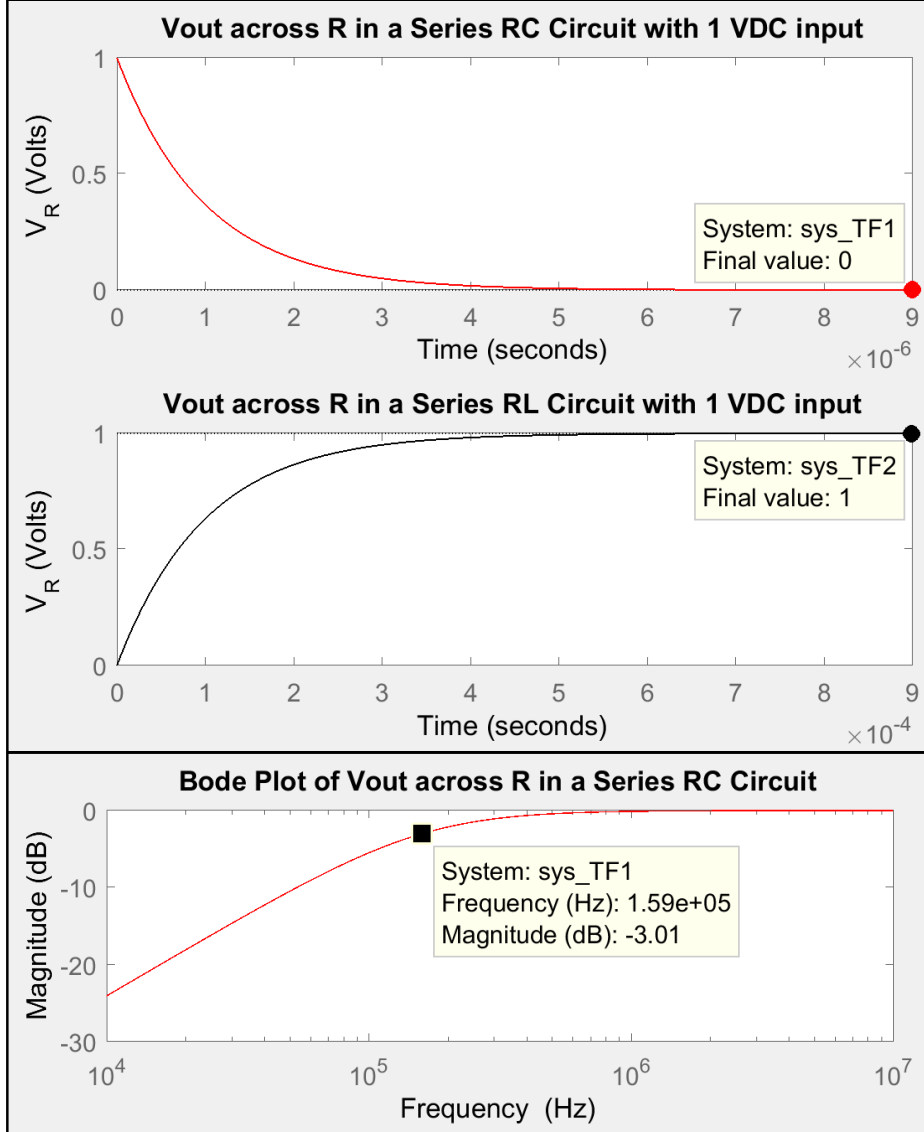

Bode Plot of Vout across R in a Series RL Circuit

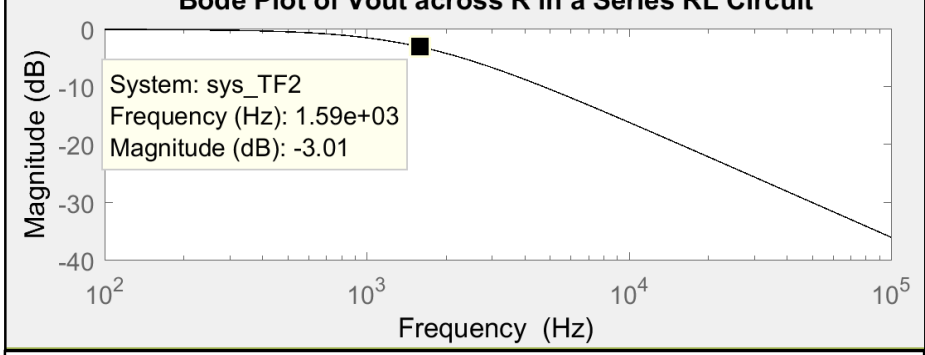

Figure A.1 - Step Response and Bode Plots for Series RC Circuit with $R=100 \Omega, C=0.01 \mu F$ and Series RL Circuit with $R=100 \Omega, L=10 \mathrm{mH}$.
$\%$ Step response and Bode Magnitude Plot of a RC and $\mathrm{RL}$ series circuits

$C=0.01 \mathrm{e}-6$;

$\mathrm{L}=10 \mathrm{e}-3$;

$\mathrm{R}=100$

syms $\mathrm{S}$

$\mathrm{Zt} 1=\mathrm{R}+1 /\left(\mathrm{C}^{*} \mathrm{~s}\right)$

$\mathrm{Zt} 2=\mathrm{R}+\mathrm{L}^{*} \mathrm{~s}$;

Vin_s $=1 ; \%$ Laplace Transform of delta function

Vout1_s = Vin_s*R/Zt1; \%VDR

Vout2_s = Vin_s*R/Zt2; \%VDR

\% Since Vin_s is equal to 1 Vout_s is actually equal to (Vout(s)/Vin(s))

TF1_sym = Vout1_s;

TF2_sym = Vout2_s;

$\%$ Find the numerator \& denominator of TF_sym [num_TF1_sym,den_TF1_sym]=numden(TF1_sym) [num_TF2_sym,den_TF2_sym] =numden(TF2_sym) $\%$ Convert num \& den to polynomial form num_TF1 = sym2poly(num_TF1_sym); den_TF1 = sym2poly(den_TF1_sym); num_TF2 $=$ sym2poly(num_TF2_sym); den_TF2 = sym2poly(den_TF2_sym); $\%$ Find the transfer function (Vo/Vin) sys_TF1 = tf(num_TF1,den_TF1); sys_TF2 = tf(num_TF2,den_TF2); figure(1) subplot $(2,1,1)$,step(sys_TF1,' $r$ ')\%This is the step response title ('Vout across R in a Series RC Circuit with 1 VDC input'), ylabel('V_R (Volts)') subplot(2,1,2),step(sys_TF2,' $k$ ')\%This is the step response title ('Vout across $\mathrm{R}$ in a Series RL Circuit with 1 VDC input'), ylabel('V_R (Volts)') figure(2)

subplot $(2,1,1), \mathrm{h} 1$ = bodeplot(sys_TF1,'r') setoptions(h1,'FreqUnits','Hz','PhaseVisible','off') title ('Bode Plot of Vout across R in a Series RC Circuit') subplot(2,1,2),h2 = bodeplot(sys_TF2,'k') setoptions(h2,'FreqUnits','Hz','PhaseVisible','off') title ('Bode Plot of Vout across R in a Series RL Circuit') 
The following is an example of a $2^{\text {nd }}$ order LPF that is solved for in Matlab in Figure A.2. The problem is solved using the Laplace method in Matlab and the step response and Bode plot are shown below. The problem is also solved for by hand to get vout(t) (Shown in bold - called vo_calc) and the step response of vout(t) is plotted below the Matlab solution step response to show the same results are achieved. Click Here to Go Back to Module 4.

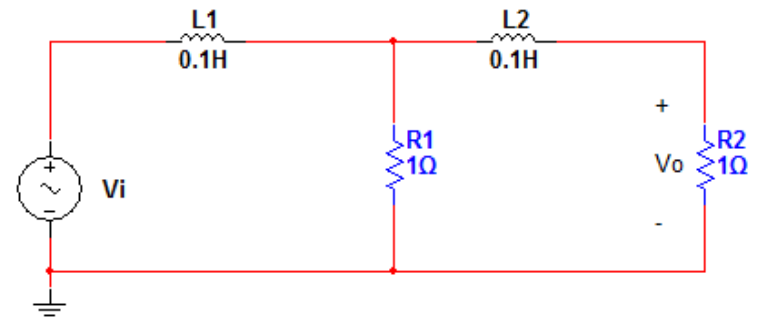

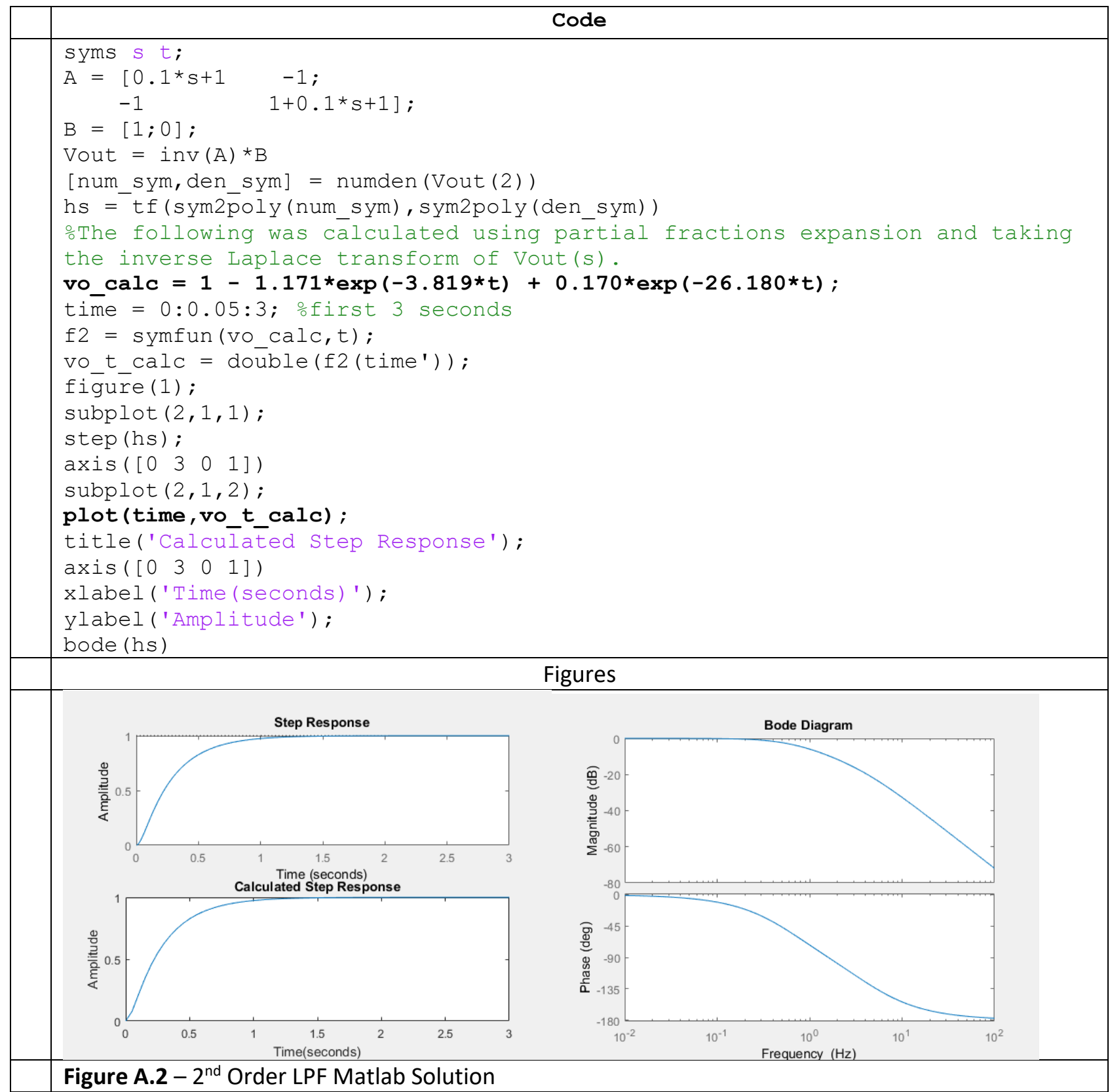


The following is an example of a $2^{\text {nd }}$ order HPF that is solved for in Matlab in Figure A.3. The problem is solved using the Laplace method in Matlab and the step response and Bode plot are shown below. The problem is also solved for by hand to get vout(t) (Shown in bold - called vo_calc) and the step response of vout(t) is plotted below the Matlab solution step response to show the same results are achieved. Click Here to Go Back to Module 4.

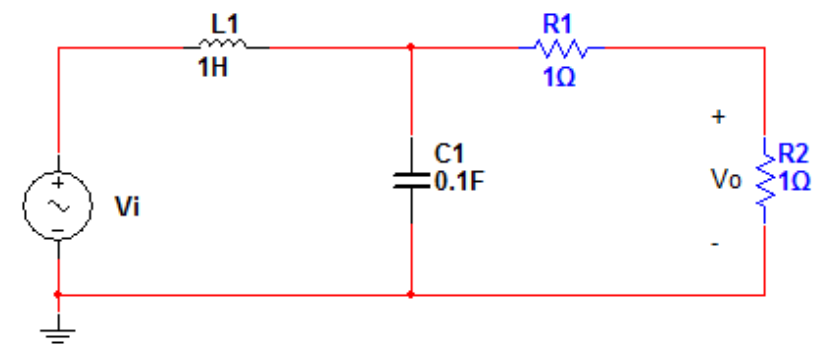

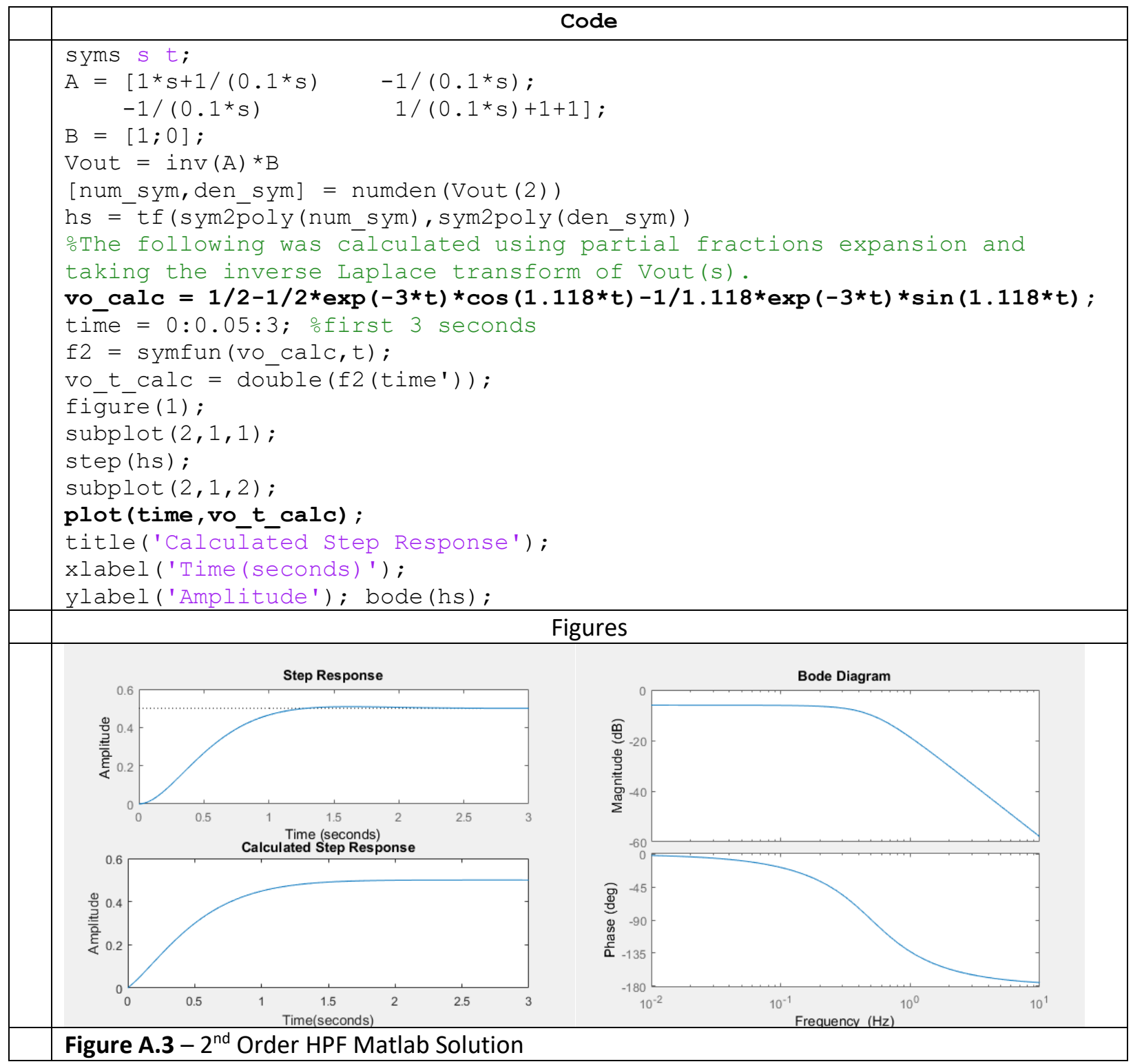


Figure A.4 shows an example of an active filter. This filter is a $4^{\text {th }}$ order Butterworth LPF filter with a $3 \mathrm{db}$ frequency at approximately $1 \mathrm{kHz}$. The Bode magnitude plot is shown. Since it is a $4^{\text {th }}$ order filter (4 capacitors are present) the slope of the stop band increases from $-20 \mathrm{~dB} / \mathrm{dec}$ for a $1^{\text {st }}$ order LPF (as described in Module 4) to $-80 \mathrm{~dB} / \mathrm{dec}$. This means that at $10 \mathrm{kHz}$ (on decade beyond the $-3 \mathrm{~dB}$ point) the gain will drop to approximately $-83 \mathrm{~dB}$. Each of the OpAmps is a $2^{\text {nd }}$ order LPF and when two are cascaded in series it produces a $4^{\text {th }}$ order filter. A third OpAmp could be included after the $2^{\text {nd }}$ OpAmp to add gain so that the pass band is not limited to $0 \mathrm{~dB}$ (or $1 \mathrm{~V} / \mathrm{V}$ ) like it is for passive RLC filters. Another important application for test and measurement equipment where multiple OpAmps are used is the instrumentation amplifier shown in Figure A.5.

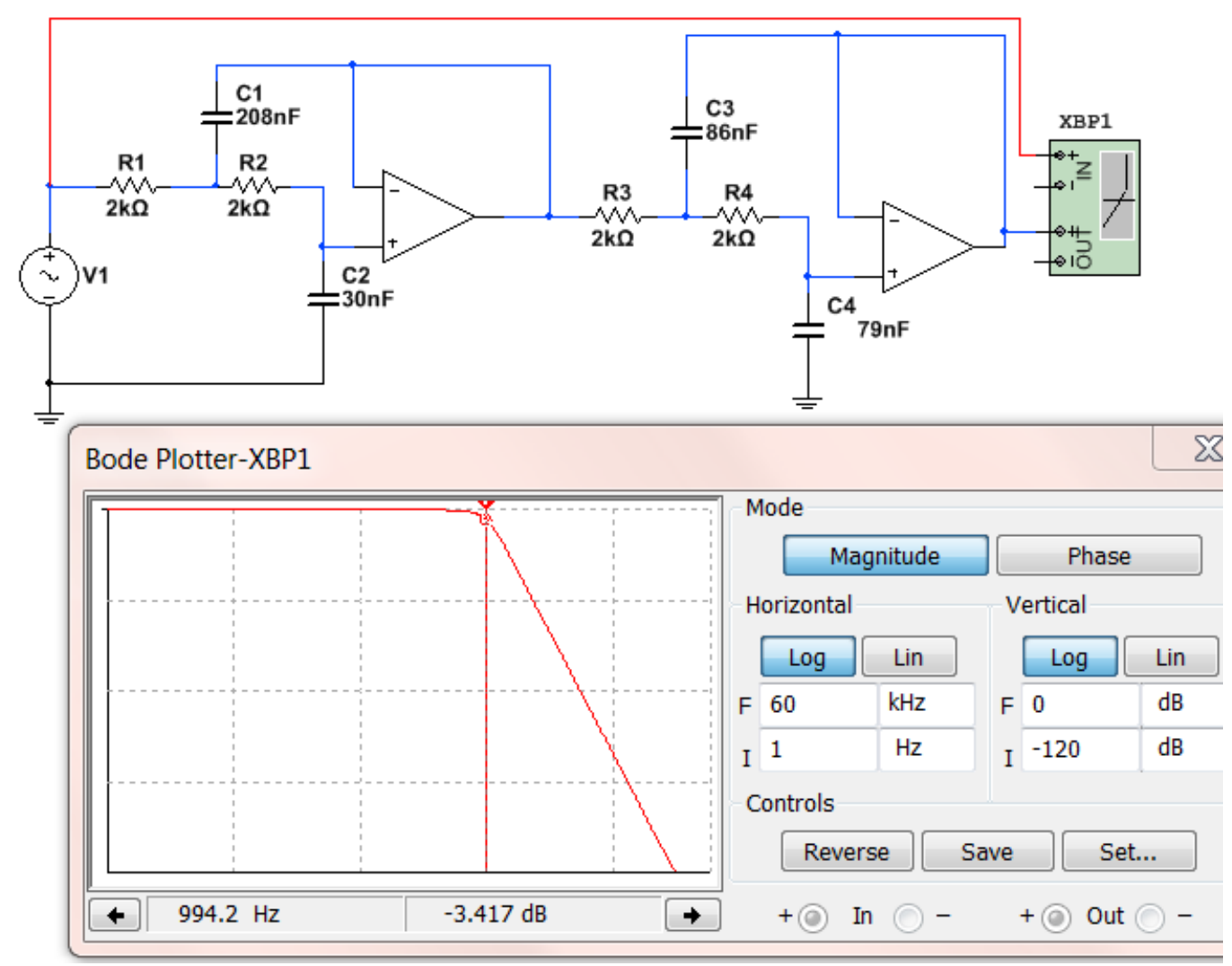

Figure A.4: $4^{\text {th }}$ Butterworth LPF with a $3 \mathrm{~dB}$ frequency at approximately $1 \mathrm{kHz}$.

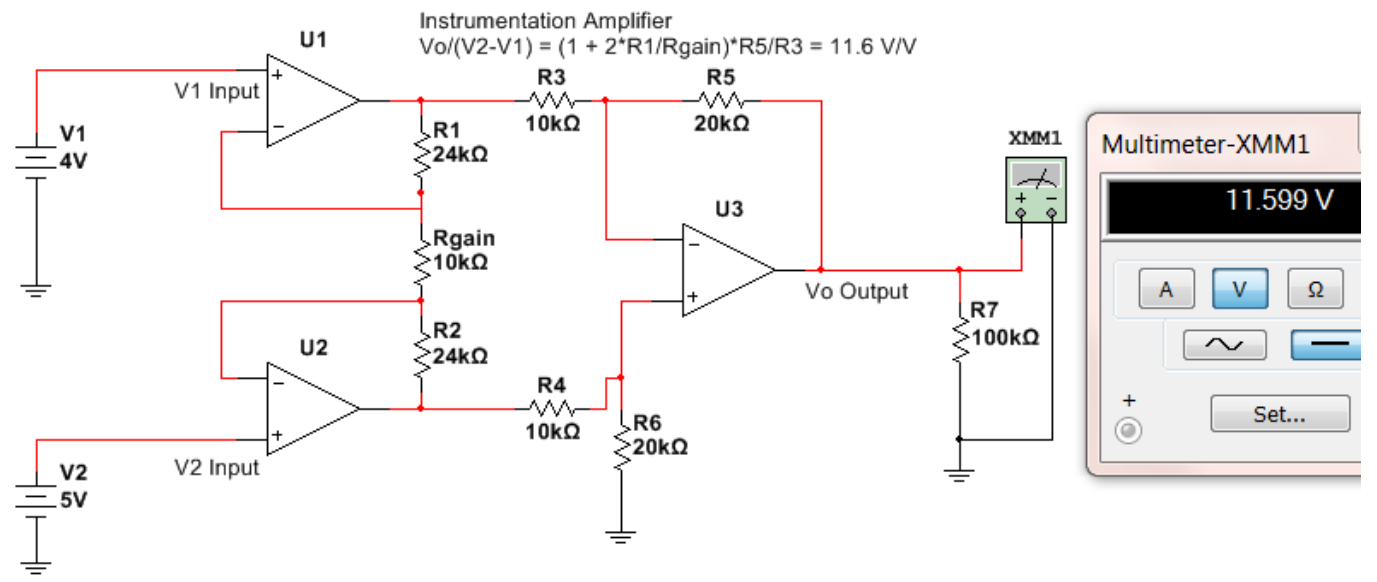

Figure A.5: Instrumentation Amplifier - useful for test and measurement equipment. 


\section{Matlab Code for Figure 1.7}

\% Plotting a square wave and triangle wave from sinusoids

$\mathrm{N}=10 ; \%$ Number of sinusoidal terms

$\mathrm{fo}=60 ;$ Fundamental Frequency

$\mathrm{T}=1 / \mathrm{fo} ; \mathrm{\circ}$ Period

$A=1 ; \%$ Amplitude

Num_T $=3$; $\%$ Number of periods to plot

Num pts per period $=500$; $\frac{\circ}{\circ}$ of points per period

tfinal = Num_T*T;

$d t=\mathrm{T} /$ Num_pts_per_period;

$t=0: d t: t \bar{f} i n a \bar{l} ;$

$\mathrm{sq}=0 \star t$

tr $=\mathrm{sq}$;

for $j=1: N-1$

$\mathrm{k}=2 * j-1$; onhis is the harmonic for the square wave

$k_{-} t=2 *(j-1)+1$; $\frac{0}{T}$ This is the harmonic for the triangle wave

$\mathrm{sq}=\mathrm{sq}+(\mathrm{A} * 4 / \mathrm{pi}) * \sin (2 * \mathrm{pi} * \mathrm{fo} * \mathrm{t} * \mathrm{k}) / \mathrm{k}$

end

$\operatorname{tr}=\operatorname{tr}+(-1)^{\wedge} j *\left(A^{*} 8 / p i^{\wedge} 2\right){ }^{*} \sin \left(2 * p i * f o * t * k \_t\right) /\left(k_{-} t^{\wedge} 2\right) ;$

\% The plot is set up to manually change the last digit on the subplot...

\% command to override the figure with a different number of terms.

subplot $(2,2,1)$,

$\operatorname{plot}\left(t, s q, k^{\prime}\right)$

hold

plot (t, tr, 'r')

hold off

grid on

title ('10 sinusoidal terms') \% Change this to match $N$ $\operatorname{axis}([0,0.05,-1.2,1.2])$ 


\section{Diode Example Problems}

1) What is the current limit of the red and green LEDs that we use in the lab and the ones that are used in the example circuits in the Davis DC Circuits book? Put answer in mA. 20mA

2) The assumed constant drop model forward bias voltage drops for different types of diodes are given in the diodes lab template. Which of the following is NOT CORRECT?
a. $1 \mathrm{~N} 914=0.7 \mathrm{~V}$
b. Red LED $=1.8 \mathrm{~V}$
c. Green LED $=2.1 \mathrm{~V}$
d. $B A T 85=0.7 \mathrm{~V}$
e. $1 \mathrm{~N} 4007=0.7 \mathrm{~V}$

3) If you use the assumed forward bias voltage drops for the constant drop model, what is the current flowing through a $1 \mathrm{~N} 914$ switching diode if the anode is connected to a $9 \mathrm{~V}$ battery and the cathode is connected to one leg of a $1 \mathrm{k} \Omega$ resistor and the other leg of the resistor is connected to ground? Put answer in $\mathrm{mA}$. (9-0.7)/1 $\mathrm{k}=\underline{\mathbf{8 . 3} \mathrm{mA}}$

4) If you put a square wave through a diode what type of current limit are you concerned with?
a. Continuous
b. DC
c. Periodic
d. Repetitive

5) The following figure shows a sketch of what you might see on the scope if a square wave input (Vi) is connected to the anode of a diode and the output (Vo) is connected to the cathode of the diode. What is the section called that is labeled with a line with arrows on its tail and head? Hint: Your answer should include 3 words that are spelled correctly. Reverse Recovery Time

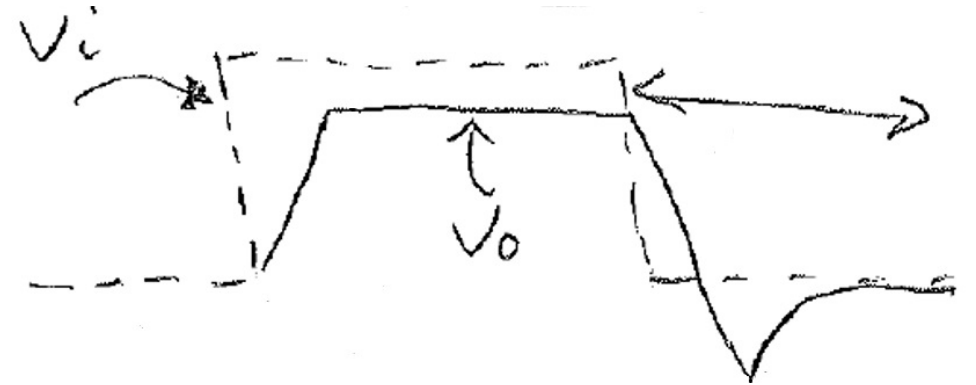

6) How many diodes would you need if you were going to build an AND gate using diode logic? 2

7) When using switches as inputs and the switch is open it is called floating. To avoid this you should always force the signal to either ground using a Pull-Down resistor or force the signal to the positive rail voltage using a Pull-Up resistor. 
8) This is a Zener regulator problem that is not covered in this book, but it is a very useful circuit. If $\mathrm{Rd}$ in the Zener regulator is $100 \Omega$, what is the minimum value of RL where the circuit continues to regulate at approximately $9 \mathrm{~V}$ ? You can assume for this problem that the voltage across the Zener diode stays at $9 \mathrm{~V}$ as long as current flows through it in the correct direction.

If $\mathrm{RL}$ is infinity (or not present) you get the maximum possible current flowing through the Zener. $I_{\max }=(12-9) / 100=30 \mathrm{~mA}$. Notice the $100 \Omega$ resistor sets how much maximum current you have in the circuit. If the maximum current flowing in the circuit is $30 \mathrm{~mA}$ then the current through RL can't be greater than 30 $\mathrm{mA}$. The worst case (min. resistor) when $\mathrm{I}_{\mathrm{RL}}=30 \mathrm{~mA}$ is when $\mathrm{RL}=$ $9 / 30 \mathrm{~mA}=\underline{300 \Omega}$

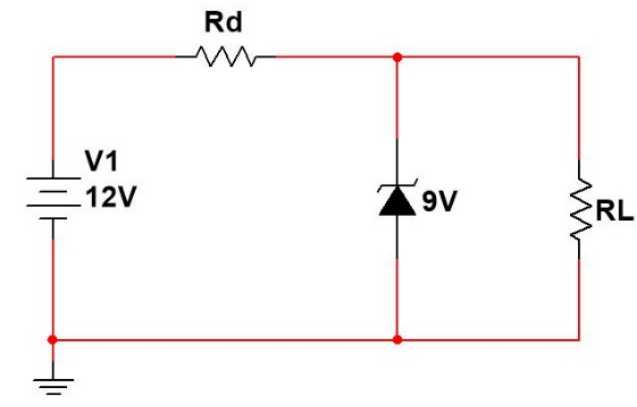

9) If the ripple voltage in the following circuit is $2 \mathrm{~V}$, what is the average (or $\mathrm{DC}$ ) voltage? Assume the transformer is ideal. $14.57 \mathrm{~V}$ (see solution below)

10) In the following circuit what resistance value would you need to get a ripple voltage of $2 \mathrm{~V}$ ? Assume the transformer is ideal. $648.8 \Omega$ (see solution below)

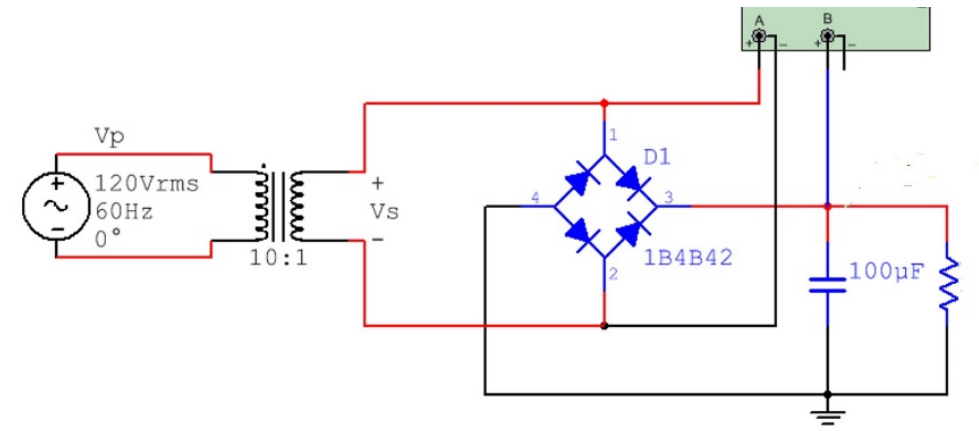

Answers to problems 9 and 10

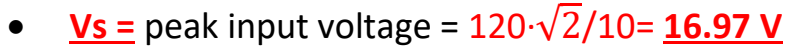

- $\underline{\text { Vo,pk }}=$ peak output voltage $=\mathrm{Vs}-2 \cdot 0.7 \mathrm{~V}=\underline{15.57 \mathrm{~V}}$

- $\quad \underline{\mathrm{Vr}}=$ ripple voltage $\left(\mathrm{Vo}_{\mathrm{o}} \mathrm{pk}-\right.$ minimum $\left.\mathrm{Vout}\right)=\frac{V_{O, p k}}{2 f R C}=\frac{15.57}{2 \cdot 60 \cdot 648.8 \cdot 100 \mu}=\underline{2 \mathrm{~V}} \leftarrow$ Given

- $\underline{\mathrm{V}}_{\mathrm{O}, \mathrm{DC}}=$ the approximate $\mathrm{DC}$ voltage of the output (centerline of the ripple). is equal to $15.57-0.5 \cdot \mathrm{Vr}=$ $14.57 \mathrm{~V}$.

11) If you connect a battery, resistor, and LED in series it is good idea to connect the anode of the LED to ground if you want it to light up. FALSE - It should be cathode (that is the negative side). Current flows through the diode from anode to cathode. Note: Zener diodes allow current to flow in both directions.

12) The term "test impedance" in a zener diode datasheet can be used to help you figure out the slope in the zener region of the zener diode characteristic curve. True - See page 2 of the Zener diode notes. Test impedance $=Z_{z}$ and the slope of the Zener region is $=1 / Z_{z}$ We often treat the Zener region like we treat the forward bias region in the constant drop model and just assume the Zener voltage is equal to a constant voltage (For a Zener this is the test voltage, $\mathrm{V}_{\mathrm{T}}$ ) and accept that it will be slightly wrong because the voltage changes slightly as the current changes (according to the slope, $1 / \mathrm{Z}_{\mathrm{z}}$ ). 
13) True or False: In a half wave rectifier circuit (like the one shown in the figure below) the negative peaks can be passed through instead of the positive peaks by simple reversing the diode direction. TRUE

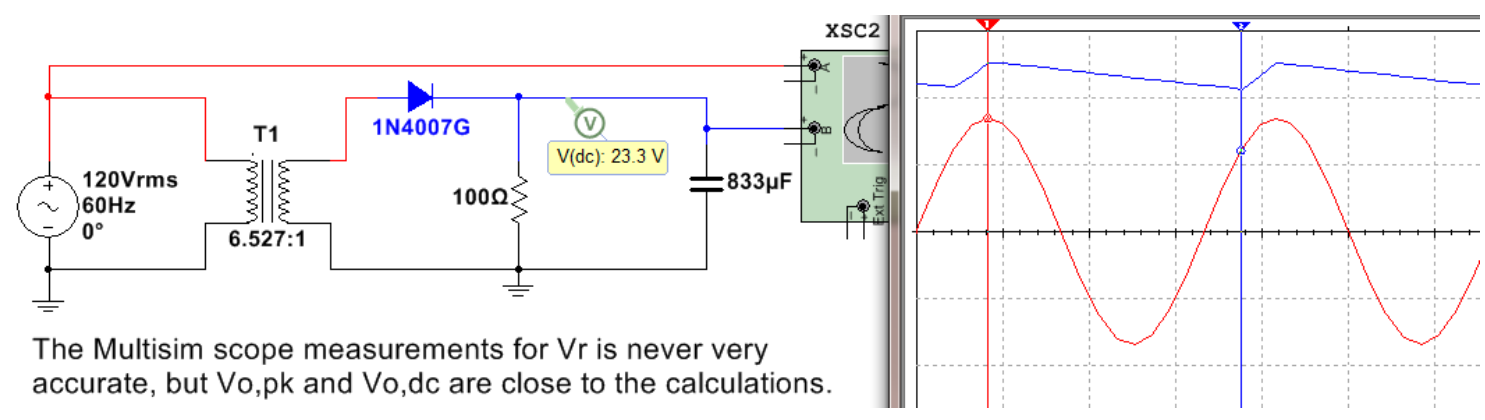

14) To measure the voltage across the resistor in a Full wave bridge rectifier with a oscilloscope that doesn't have differential probes you need to put two probes on the two sides of the resistor, connect the ground clips to ground, and us the Math Function A + B. FALSE - You should use the A-B function.

15) DC Diode Biasing Problem. I am giving you some information to make this problem faster to solve. If it is known that the following circuit has a XMM3 reading of $190.9 \mathrm{~mA}$ and a XMM4 reading of $181.8 \mathrm{~mA}$. Show the diode current and voltage for each of the diodes and verify forward or reverse bias.

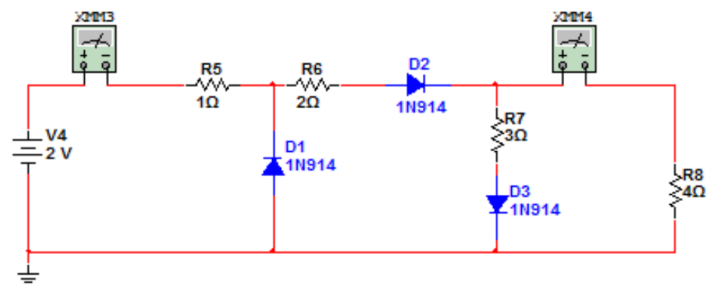

Assume D1 is reverse biased \& D2 and D3 are forward biased.

Use the Mesh matrix method to solve for 11 and 12 (see Module 2 of DC Circuits for help on this).

$|1+2+3 \quad-3 \quad||11|=|-0.7|$

$\left|\begin{array}{lll|l|}\mid & -3 & 3+4|| 12 \mid & |+0.7|\end{array}\right|$

$\mathrm{I} 1=\mathrm{I}_{\mathrm{D} 2}=0.1909 \mathrm{~A}$ and $\mathrm{I} 2=0.1818 \mathrm{~A} \rightarrow \mathrm{I}_{\mathrm{D} 3}=\mathrm{I} 1-\mathrm{I} 2=0.0091 \mathrm{~A}$

Both ID2 and ID3 have currents flowing from anode to cathode, so forward bias checks out.

D1 - Check for Reverse Bias

KVL around $1^{\text {st }}$ loop: $-2+1 * 0.1909=-1.81 \mathrm{~V}$

Since $V_{D 1}$ is less than $0.7 \mathrm{~V}$, reverse bias checks out. 
16) If you built a Full-Wave Bridge rectifier (without using a transformer) with a $1 \mathrm{k} \Omega$ resistor and a $4.7 \mu \mathrm{F}$ capacitor to convert an AC input voltage of 30 Volts peak to peak at $1 \mathrm{kHz}$ to a DC voltage what approximate DC voltage (using the DC voltage equation in the notes) would you get across the load resistor? Also, determine the Peak Revers Voltage (or Peak Inverse Voltage) across each of the 4 diodes in this circuit.

$$
\begin{aligned}
& \mathrm{RL}=1 \mathrm{k} \Omega \quad \mathrm{C}=4.7 \cdot 10^{-6} \mathrm{~F} \quad \mathrm{~F}=1 \mathrm{kHz} \\
& \mathrm{Vs}, \mathrm{pk}=15 \mathrm{Vpk} \\
& \mathrm{Vo}, \mathrm{pk}=15-2 \cdot 0.7=\underline{\mathbf{1 3 . 6 \mathrm { V }}} \\
& \mathrm{Vr}=\mathrm{Vo}, \mathrm{pk} /(2 \mathrm{FRC})=13.6 /\left(2 \cdot 1000 \cdot 1000 \cdot 4.7 \cdot 10^{-6}\right)=\underline{\mathbf{1 . 4 5} \mathrm{V}} \\
& \mathrm{V}_{\mathrm{O}, \mathrm{DC}}=\mathrm{Vo}, \mathrm{pk}-1 / 2 \cdot \mathrm{Vr}=13.6-1.45 / 2=\underline{\mathbf{1 2 . 8 8} \mathrm{V}}
\end{aligned}
$$




\section{OpAmp Example Problems}

1) Design an OpAmp circuit with an adjustable gain using only a $10 \mathrm{k} \Omega$ potentiometer, a $1 \mathrm{k} \Omega$ fixed resistor, and one other fixed resistor. The input voltage is a $1 \mathrm{~V}$ peak sinusoid $(\mathrm{Vi}(\mathrm{t})=1 \sin (\omega \cdot \mathrm{t}))$ and the output ranges from $5 \mathrm{~V}$ peak to $15 \mathrm{~V}$ peak $(\operatorname{Vo}(t)=5 \cdot \sin (\omega \cdot t)$ to $15 \cdot \sin (\omega \cdot t))$. Draw the circuit in the space below and list the value of the "one other fixed resistor".

It must be a non-inverting configuration OpAmp circuit with the resistor equal to $\underline{4 \mathrm{k} \Omega}$

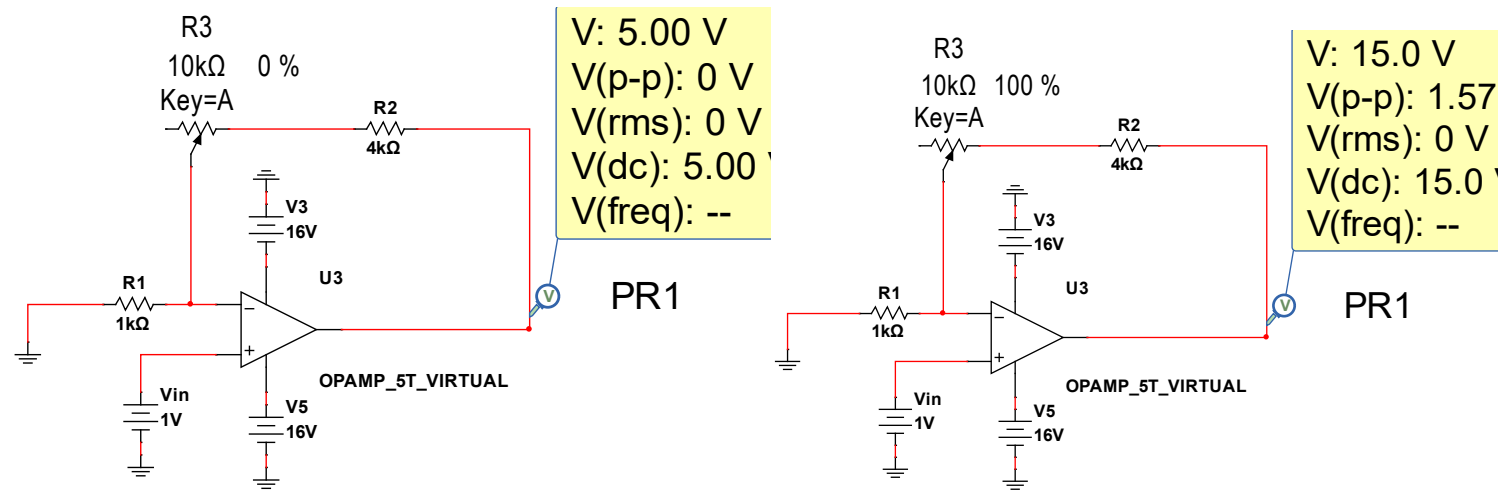

2) If you wanted to achieve a gain of $8 \mathrm{~V} / \mathrm{V}$ gain and the OpAmp has a unity gain bandwidth of $1 \mathrm{MHz}$ and your input voltage to the OpAmp is $\mathrm{Vi}(\mathrm{t})=2 \cdot \sin (2 \cdot \pi \cdot 10,000 \cdot \mathrm{t})$ what resistor ratio $\mathrm{R} 2 / \mathrm{R} 1$ would you use. You also want the output in phase with the input. $\underline{\mathrm{R} 2 / \mathrm{R} 1=7.7}$

Find Ao first. Ao $=\mathrm{Ft} / \mathrm{Fop}=1 \mathrm{M} / 10 \mathrm{k}=100 \mathrm{~V} / \mathrm{V}$ (If you get this you get 1 point.

$8=[1+R 2 / R 1] /[(1+(1+R 2 / R 1) / A o)]$

$8(1+(1+R 2 / R 1) / 100)=1+R 2 / R 1 \rightarrow 8+.08+.08(R 2 / R 1)=1+R 2 / R 1 \rightarrow 7.08=0.92 R 2 / R 1$

$\mathrm{R} 2 / \mathrm{R} 1=7.7$

3) If the unity gain bandwidth of the OpAmp in the following circuit is $1 \mathrm{MHz}$ and $\mathrm{Vi}=0.5 \sin (2 \pi \cdot 50,000 \mathrm{t})$, what is the peak to peak output voltage? Be careful on peak voltage versus peak to peak voltage! Since the input frequency is high you CANNOT assume it is ideal.

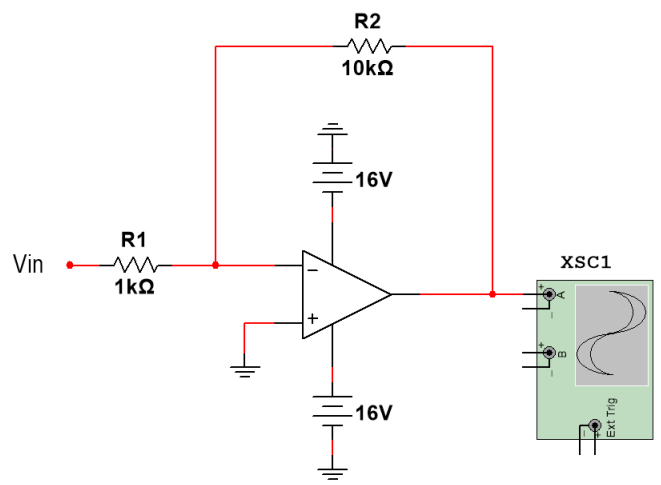

$$
\begin{aligned}
& \text { Vi,pk }=0.5 . V i, p-p=1 \mathrm{~V} \\
& \text { Ao }=f t / f o p=1 M / 50 k=20 \mathrm{~V} / \mathrm{V} \\
& \mathrm{Vo,p-p}=\mathrm{Vi}, \mathrm{p}-\mathrm{p}^{*}|(-\mathrm{R} 2 / \mathrm{R} 1) /[1+(1+\mathrm{R} 2 / \mathrm{R} 1) / \mathrm{Ao}]| \\
& \mathrm{Vo,p-p}=1^{*}|(-10) /[1+(1+10) / 20]|=\underline{6.45 \mathrm{Vp}-\mathrm{p}} \\
& \text { Note: if the ideal assumption would have been made } \\
& \text { the output would have been way off } \rightarrow 1 * 10=10 \mathrm{~V} \text {-p }
\end{aligned}
$$


4) If the potentiometer in the circuit below is turned to ZERO \% so that no potentiometer resistance is in the circuit, what will the voltage across $\mathrm{RL}$ be equal to?

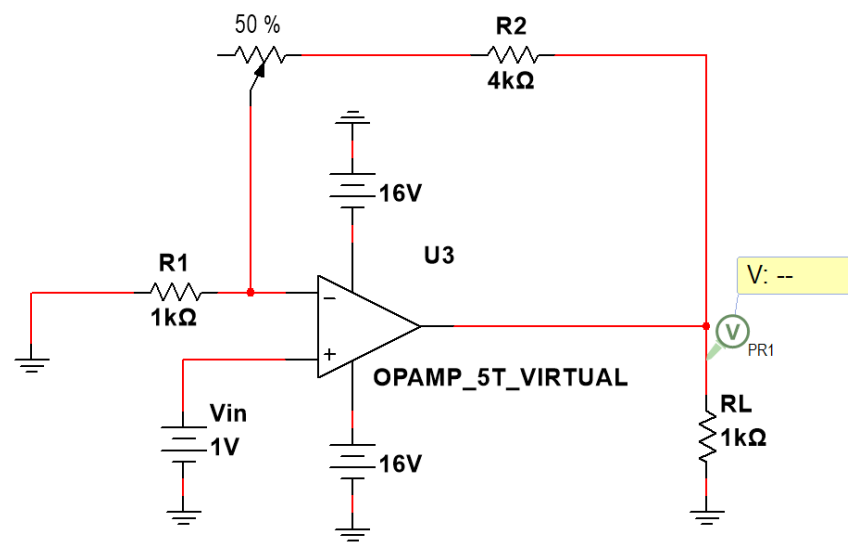

Non-inverting OpAmp

$\mathrm{Vo}=1 *(1+\mathrm{R} 2 / \mathrm{R} 1)=1 *(1+4)=\underline{\mathbf{5 V}}$

5) Assuming the following OpAmp can be treated as ideal, what size of potentiometer would be needed for the value of PR1 in the circuit below to read $5.5 \mathrm{~V}$. Note: $50 \%$ means that one half of the total potentiometer resistance is in the circuit.

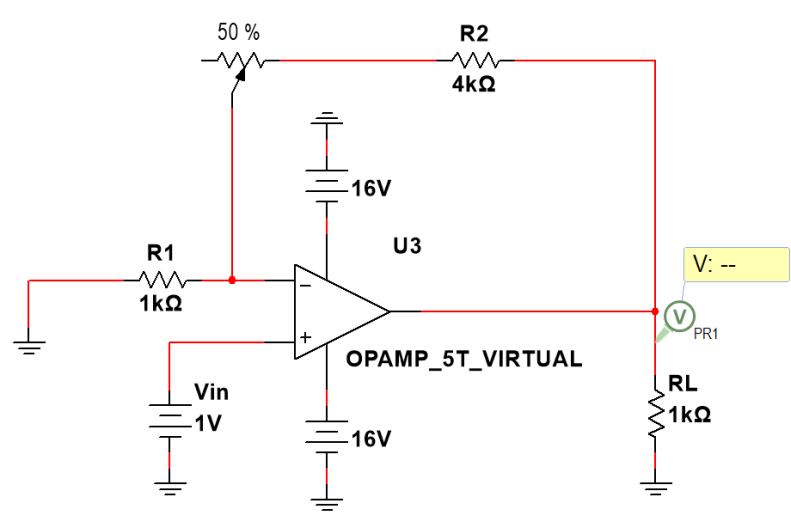

$$
\begin{aligned}
& \text { Non-inverting OpAmp } \\
& \text { Vo }=5.5=1^{*}(1+\mathrm{R} 2 / \mathrm{R} 1)=1 * 1+(4 \mathrm{k}+0.5 \mathrm{Rx}) / 1 \mathrm{k} \\
& \text { Solving for } \mathrm{Rx} \rightarrow \mathrm{Rx}=\underline{\mathbf{1 k} \Omega}
\end{aligned}
$$

6) If a Butterworth low pass Active OpAmp filter had a passband gain of $200 \mathrm{~V} / \mathrm{V}$ what is that gain in $\mathrm{dB}$ ? $20 * \log 10(200)=\underline{46 \mathrm{~dB}}$ 